DOE/ER 25232-T

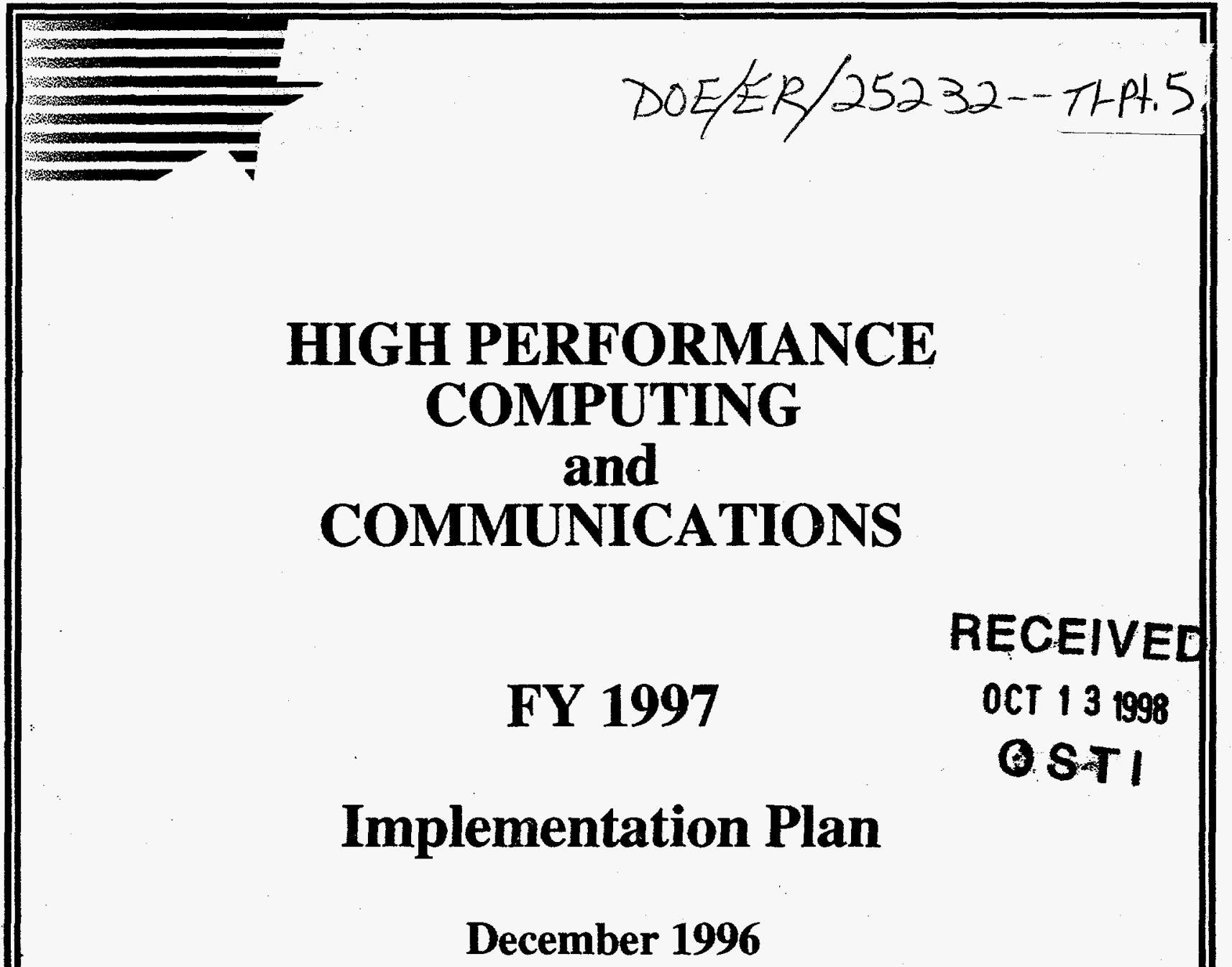

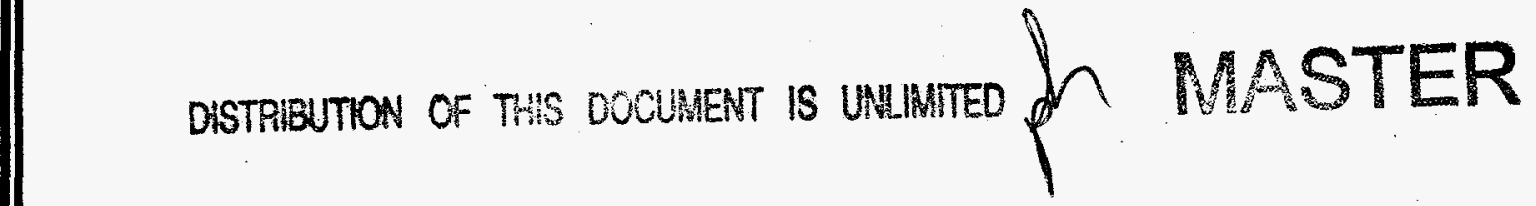

National Coordination Office for Computing, Information, and Communications Executive Office of the President

National Science and Technology Council 


\section{DISCLAIMER}

This report was prepared as an account of work sponsored by an agency of the United States Government. Neither the United States Government nor any agency thereof, nor any of their employees, make any warranty, express or implied, or assumes any legal liability or responsibility for the accuracy, completeness, or usefulness of any information, apparatus, product, or process disclosed, or represents that its use would not infringe privately owned rights. Reference herein to any specific commercial product, process, or service by trade name, trademark, manufacturer, or otherwise does not necessarily constitute or imply its endorsement, recommendation, or favoring by the United States Government or any agency thereof. The views and opinions of authors expressed herein do not necessarily state or reflect those of the United States Government or any agency thereof. 


\section{DISCLAIMER}

Portions of this document may be illegible in electronic image products. Images are produced from the best available original document. 
Suite $665 \bullet 4201$ Wilson Boulevard • Arlington, Virginia 22230

(703) 306-HPCC・FAX (703) 306-4727•nco@ hpcc.gov • http://www.hpcc.gov/

December 30, 1996

Dr. John H. Gibbons

Director, Office of Science and Technology Policy

Executive Office of the President

Old Executive Office Building

Washington, D. C. 20500

Dear Dr. Gibbons:

I am delighted to forward you the FY 1997 Implementation Plan for the High Performance Computing and Communications Program. This document describes in detail the activities planned by the twelve participating HPCC Agencies to advance the overall objectives of the Program.

The HPCC Program celebrated its fifth anniversary in October 1996, with an impressive array of significant accomplishments to its credit, many of which are described in this year's report, "Advancing the Frontiers of Information Technology: Supplement to the President's FY 1997 Budget." Both this report and the Implementation Plan have been endorsed by the Committee on Computing, Information, and Communications R\&D of the National Science and Technology Council.

The detailed descriptions of the planned budgetary commitments are similar to previous year's plans and were determined through substantial interaction with the Office of Management and Budget. This document will be made available to the public in printed form and can also be accessed via the NCO World Wide Web home page at http://www.hpcc.gov.

On behalf of all the participating HPCC Agencies, the knowledgeable professionals serving in these agencies, and the many helpers and advisors from academia, industry, and the public, please accept my thanks for your leadership of this effort.

Enclosure

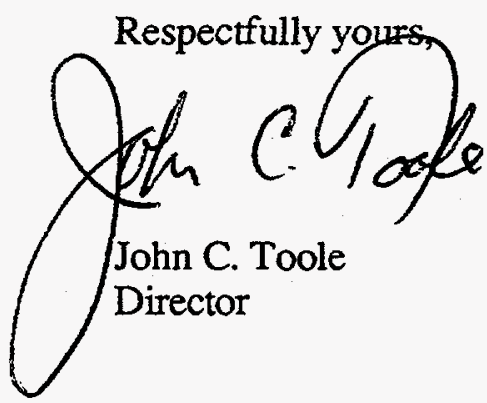





\section{HPCC FY 1997 Implementation Plan}

Table of Contents

1. Executive Summary .

2. Introduction

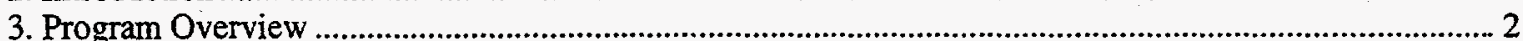

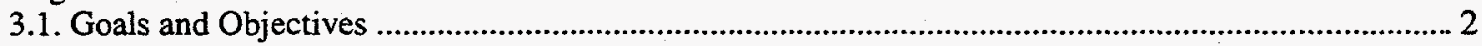

3.2. Definition of Program Component Areas ....................................................................................................2

3.3. HPCC Management, Planning and Organization .......................................................................... 3

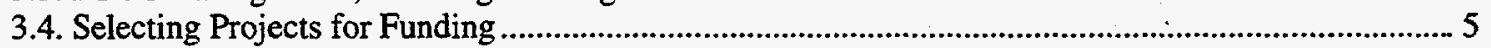

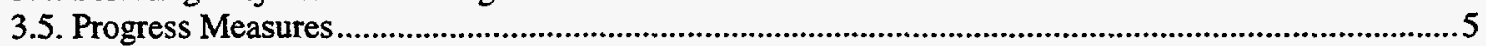

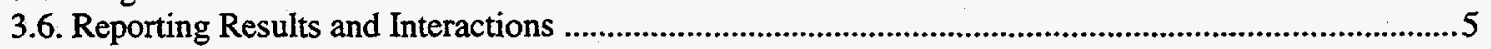

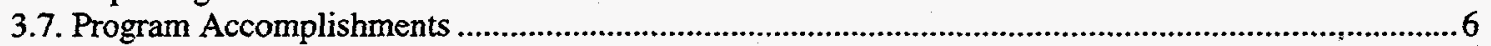

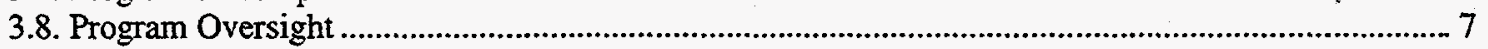

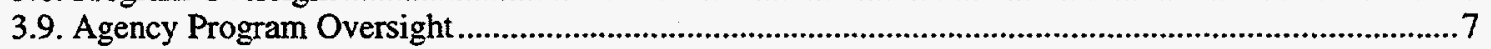

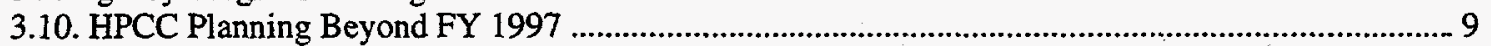

4. HPCC FY 1997 Budget Overview by Program Component Areas ........................................................... 10

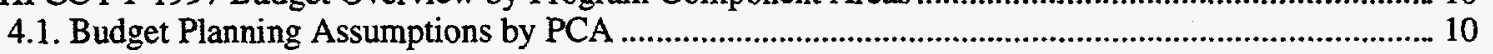

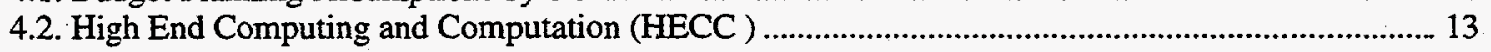

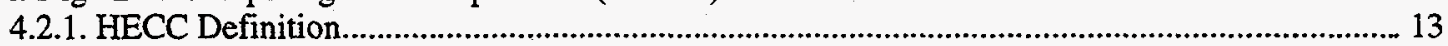

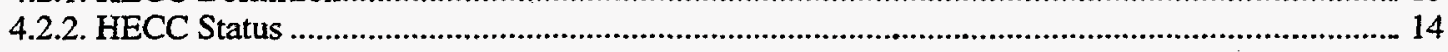

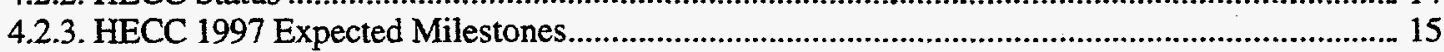

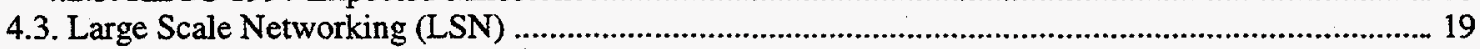

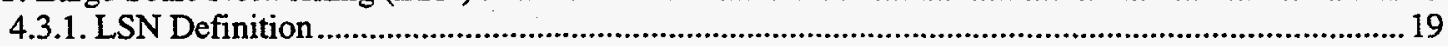

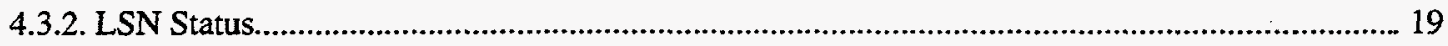

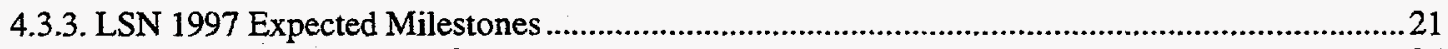

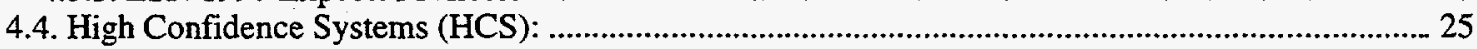

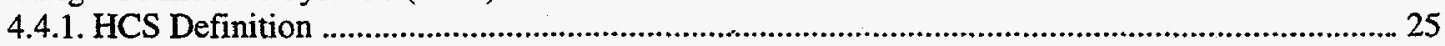

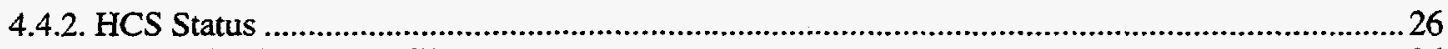

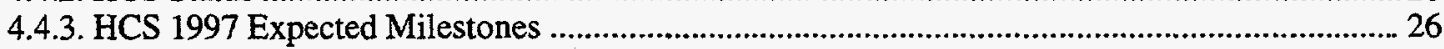

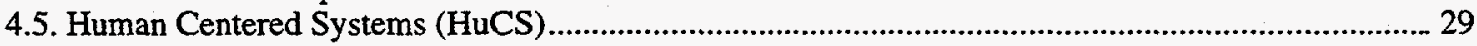

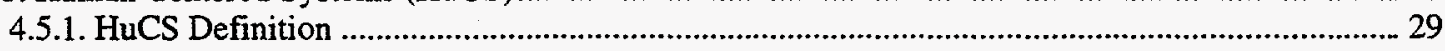

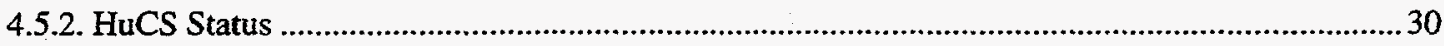

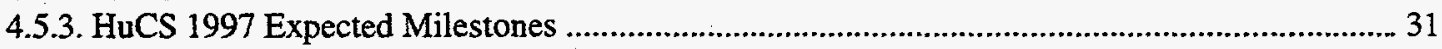

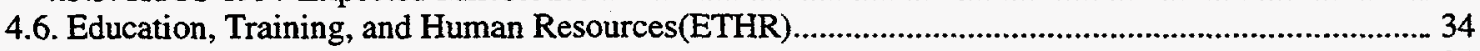

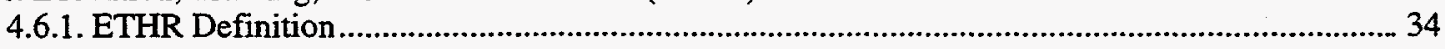

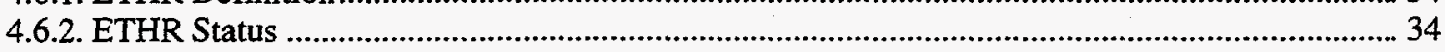

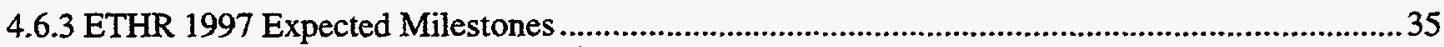

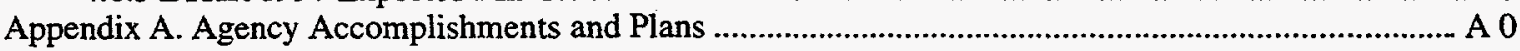




\section{List of Tables}

Table

Table 1: HPCC FY 1997 IP Budget Summary

Table 1A: HPCC Budget Comparisons for FY 1997

Table 2: HECC Program Activity Summary

Table 3: LSN Program Activity Summary

Table 4: HCS Program Activity Summary

Table 5: HuCS Program Activity Summary

Table 6: ETHR Program Activity Summary

\section{Appendix A}

DARPA Program Activity Summary

NSF Program Activity Summary

DOE Program Activity Summary .

NASA Program Activity Summary. A65

NIH Program Activity Summary.

NSA Program Activity Summary

NIST Program Activity Summary. A106

NOAA Program Activity Summary

EPA Program Activity Summary 


\title{
High Performance Computing and Communications Program
}

\author{
FY 1997 Implementation Plan
}

\section{Executive Summary}

Based on the President's budget request of over $\$ 1$ billion, this document presents in detail the FY 1997 Implementation Plan for the Federal High Performance Computing and Communications (HPCC) Program. Established by the High Performance Computing Act 1991, the Program is carried out by twelve Federal agencies. ${ }^{1}$ Throughout its existence, this Program has achieved extraordinary results relative to the original goals established in FY 1992.

The budget figures included in this Implementation Plan derive from a base of $\$ 489$ million in place at the beginning of the official Program in FY 1992. Additional funding was added over the following five years.

Since the HPCC Program is part of the Administration's R\&D portfolio, program oversight is the responsibility of the National Science and Technology Council's (NSTC) Committee on Computing, Information, and Communications R\&D (CCIC). In FY 1996, the High Performance Computing, Communications, and Information Technology (HPCCIT) Subcommittee became the Computing, Information, and Communications R\&D (CIC) Subcommittee of the CCIC and is currently responsible for coordinating agencies' collaborative R\&D activities.

The HPCC Program forms the core of the CIC R\&D programs. Starting with this fiscal year, CCIC has reorganized its collaborative programs into five Program Component Areas (PCAs) whose structure evolved from the five components of the original HPCC Program (HPCS, NREN, ASTA, IITA, and BRHR) ${ }^{2}$. The PCAs are areas of high priority investments by the Federal agencies that participate in the coordinated R\&D programs. The PCAs and their goals are:

High End Computing and Computation (HECC): The goal of HECC R\&D is to provide the foundation for U.S. leadership in computing through investments in leading-edge hardware and software innovations, and in algorithms and software for modeling and simulation needed to address Grand Challenge-class applications.

Large Scale Networking Technologies (LSN): The goal of LSN R\&D is to assure U.S. leadership in communications in high performance network components; technologies that enable wireless, optical, mobile, and wireline communications; large-scale network engineering, management, and services; and systems software and program development environments for network-centric computing.

High Confidence Systems (HCS): The goal of HCS R\&D is to develop technologies that provide users with high levels of security, protection of privacy and data, reliability, and restorability of information services.

Human Centered Systems (HuCS): The goal of HuCS R\&D is to make computing and networking more useful through collaboratories, technologies that provide knowledge from distributed repositories, multimodal interactive systems, and virtual reality environments.

Education, Training, and Human Resources (ETHR): The goal of ETHR R\&D is to support research that enables modern education and training technologies, including technologies that support lifelong and distance learning, and curriculum development.

\footnotetext{
${ }^{1}$ DARPA, NSF, DOE, NASA, NIH, NSA, NIST, NOAA, EPA, ED, AHCPR, and VA. DOT may be added to the Program later.

${ }^{2}$ High Performance Computing Systems (HPCS), National Research and Education Network (NREN), Advanced Software Technology and Algorithms (ASTA), Information Infrastructure Technology and Applications (IITA), and Basic Research and Human Resources (BRHR)
} 
Many applications span several PCAs, and numerous areas of research may contribute to more than one PCA. In this document, descriptions of program activities for each agency indicate which activities contribute to which PCAs.

Research directions for the FY 1997 Program include the following:

In HECC, emphasis is being placed on system software technology for high performance systems--especially scalable clusters of shared memory processors. There is new emphasis on research on fundamental technologies based on quantum effects and biological phenomena. There will be less emphasis on procurements of large-scale experimental systems this fiscal year. Re-competition of the NSF Supercomputer Centers and the DOE High Performance Computing Research Centers is taking place during this period. ${ }^{3}$

In LSN, emphasis is on very high bandwidth optical, wireline, and wireless communications, very large aggregates of very small processors, connectivity for large numbers of universities and schools, distributed cooperative computing, and applications using computer-based patient records.

In HCS, agencies focus on the high performance aspects of system reliability, provability, and privacy of sensitive unclassified data, with some emphasis on information security. As the importance of this area grows, additional R\&D activities will be supported to provide for computer based patient records, electronic commerce, and emergency management.

In HuCS, emphasis is on human-computer interaction techniques, including interactive problem-solvirig, software development technology, speech and document understanding, digital library technology, collaboratories, virtual reality applications in telemedicine, remote operation of expensive and unique equipment, and technologies required for remote collaboration.

In ETHR, closer cooperation between the NSTC Committee on Education and Training and the CCIC is planned. Some of the prior year HPCC education and training budget has been redefined and is no longer considered part of the HPCC crosscut. Education activities, such as supporting fellowships for advanced training in CCIC areas, are still high priorities for the HPCC agencies. New efforts to bring health care to remote individuals is also a high priority.

The world has entered the Information Age and the U.S. is leading the way. This leadership is due in large measure to long-term Federal research and development in advanced computing, communications, and information technology. The new CIC R\&D programs will be key driving forces for advancing these technologies and their application to a more secure and better life in 21st century America.

This Implementation Plan contains the details of FY 1997 HPCC Program activities reflected in the President's FY 1997 budget request.

${ }^{3}$ Re-competition of the DOE NERSC center was completed in FY 1996. 
FY 1997 HPCC Activities by Program Component Area

\begin{tabular}{|c|c|c|c|c|}
\hline 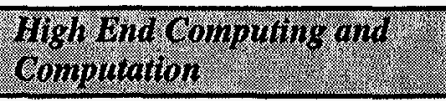 & 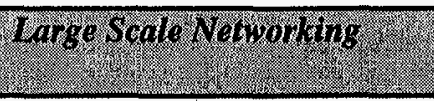 & 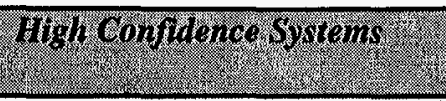 & 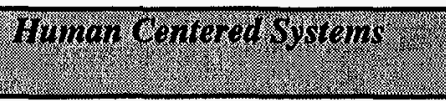 & 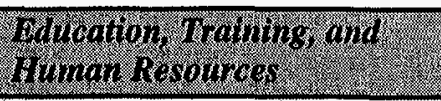 \\
\hline $\begin{array}{l}\text { Scalable systems, software, } \\
\text { and environments }\end{array}$ & $\begin{array}{l}\text { Multi-gigabit network testbed } \\
\text { including wireline, wireless, } \\
\text { and mobile technologies }\end{array}$ & $\begin{array}{l}\text { Infrastructure protocols for } \\
\text { secure and reliable networks }\end{array}$ & $\begin{array}{l}\text { Intelligent systems and } \\
\text { software }\end{array}$ & $\begin{array}{l}\text { Integration of research and } \\
\text { education technologies }\end{array}$ \\
\hline $\begin{array}{l}\text { Uniform memory access } \\
\text { systems with low latency and } \\
\text { high bandwidth }\end{array}$ & $\begin{array}{l}\text { Very high speed backbones } \\
\text { for network, scientific, and } \\
\text { engineering applications }\end{array}$ & $\begin{array}{l}\text { High speed cryptography for } \\
\text { information security in virtual } \\
\text { laboratories }\end{array}$ & $\begin{array}{l}\text { Knowledge acquisition, } \\
\text { fusion, aggregation, and } \\
\text { summarization tools }\end{array}$ & $\begin{array}{l}\text { Lifelong and distance } \\
\text { learning technologies }\end{array}$ \\
\hline $\begin{array}{l}\text { Integrated circuit simulation } \\
\text { for microsystems }\end{array}$ & $\begin{array}{l}\text { Network reconfiguration } \\
\text { tools }\end{array}$ & Interoperability standards & $\begin{array}{l}\text { Virtual environments with } \\
\text { remote instruments }\end{array}$ & Teacher development tools \\
\hline Distributed operating systems & $\begin{array}{l}\text { Global grid communications } \\
\text { and inter-network protocols }\end{array}$ & Secure interface protocols & $\begin{array}{l}\text { Multi-modal communication, } \\
\text { speech understanding }\end{array}$ & $\begin{array}{l}\text { K- } 12 \text { education cooperative } \\
\text { agreements }\end{array}$ \\
\hline $\begin{array}{l}\text { Compilers, debuggers, } \\
\text { program development } \\
\text { environments }\end{array}$ & $\begin{array}{l}\text { Network management, cost } \\
\text { accounting, and } \\
\text { authentication }\end{array}$ & $\begin{array}{l}\text { Network security and } \\
\text { restorability }\end{array}$ & $\begin{array}{l}\text { Collaboratories, tools for } \\
\text { group authoring, remote } \\
\text { control of instruments }\end{array}$ & $\begin{array}{l}\text { Summer school in high } \\
\text { performance computation }\end{array}$ \\
\hline $\begin{array}{l}\text { Algorithms and software for } \\
\text { Grand Challenge scientific } \\
\text { and engineering applications }\end{array}$ & $\begin{array}{l}\text { Network-centric computation } \\
\text { for National Challenge } \\
\text { applications }\end{array}$ & $\begin{array}{l}\text { Reliability and security for } \\
\text { mobile computing } \\
\text { environments }\end{array}$ & $\begin{array}{l}\text { Graphical user interfaces for } \\
\text { medical imaging and patient } \\
\text { record systems }\end{array}$ & $\begin{array}{l}\text { Research fellowships for } \\
\text { science, engineering, and } \\
\text { health professionals }\end{array}$ \\
\hline $\begin{array}{l}\text { Supercomputer centers, } \\
\text { centers for advanced research } \\
\text { on experimental systems }\end{array}$ & $\begin{array}{l}\text { Data mining, integration, and } \\
\text { visualization for scientific } \\
\text { data }\end{array}$ & $\begin{array}{l}\text { Telemedicine testbed } \\
\text { networks, remote surgical } \\
\text { procedures }\end{array}$ & $\begin{array}{l}\text { Clinical decision support } \\
\text { systems, patient records, } \\
\text { visible human database }\end{array}$ & $\begin{array}{l}\text { Health care information } \\
\text { infrastructure }\end{array}$ \\
\hline $\begin{array}{l}\text { Advanced manufacturing } \\
\text { design and processes } \\
\text { Superconducting components } \\
\text { Quantum and biological } \\
\text { computing methods }\end{array}$ & $\begin{array}{l}\text { Optoelectronic and optical } \\
\text { media } \\
\text { Convergence of computing } \\
\text { and communications }\end{array}$ & $\begin{array}{l}\text { Authentication and } \\
\text { verification procedures }\end{array}$ & $\begin{array}{l}\text { Remote visualization of } \\
\text { environmental data in digital } \\
\text { libraries } \\
\text { Automatic document } \\
\text { translation }\end{array}$ & $\begin{array}{l}\text { Technologies for ubiquitous } \\
\text { network access to the World } \\
\text { Wide Web }\end{array}$ \\
\hline$\$ 454$ Million & $\$ 260$ Million & $\$ 32$ Million & $\$ 249$ Million & $\$ 45$ Million \\
\hline
\end{tabular}




\section{Introduction}

The High Performance Computing and Communications (HPCC) Program was formally authorized by passage, with bipartisan support, of the High-Performance Computing Act of 1991 (Public Law 102-194), signed on December 9 , 1991. The original Program, in which eight Federal agencies participated, has now grown to twelve agencies. This Plan provides a detailed description of the agencies' FY 1996 HPCC accomplishments and FY 1997 HPCC plans.

HPCC activities are coordinated by the Computing, Information, and Communications R\&D (CIC) Subcommittee of the Committee on Computing, Information and Communications R\&D (CCIC), one of the nine committees of the National Science and Technology Council. This CIC R\&D Subcommittee is the new name for the High Performance Computing, Communication, and Information Technology (HPCCIT) Subcommittee.

In FY 1991, before the formal start of the Program, the HPCC-related activities by the original eight agencies totaled approximately $\$ 489$ million-the Program's original base level. The Program goals justified annual funding levels that were expected to grow to approximately $\$ 1,500$ million over five years. Although the funding has not grown to this level, the Program has remained a healthy and vital mechanism for R\&D in the enabling technologies required for computing, information, and communications.

The overall funding profile for the HPCC Program, is as follows:

\begin{tabular}{|c|c|c|c|c|}
\hline Fiscal Year & $\begin{array}{c}\text { Participating } \\
\text { Agencies } \\
\text { (Dollars in } \\
\text { millions) }\end{array}$ & $\begin{array}{c}\text { Change } \\
\text { from } \\
\text { Previous } \\
\text { Year }\end{array}$ & $\begin{array}{c}\text { Original } \\
\text { Eight } \\
\text { Agencies } \\
\text { (Dollars } \\
\text { in } \\
\text { millions) }\end{array}$ & $\begin{array}{c}\text { Change } \\
\text { from } \\
\text { Previous } \\
\text { Year for } \\
\text { Original } \\
\text { Eight }\end{array}$ \\
\hline $\begin{array}{c}\text { FY 1992 Actual } \\
\text { (Eight Agencies) }\end{array}$ & $\$ 655$ & $+33.9 \%$ & $\$ 655$ & $+33.9 \%$ \\
\hline $\begin{array}{c}\text { FY 1993 Actual } \\
\text { (Ten Agencies) }\end{array}$ & $\$ 795$ & $+21.4 \%$ & $\$ 783$ & $+19.5 \%$ \\
\hline $\begin{array}{c}\text { FY 1994 Actual } \\
\text { (Ten Agencies, IITA added) }\end{array}$ & $\$ 938$ & $+18.0 \%$ & $\$ 925$ & $+18.1 \%$ \\
\hline $\begin{array}{c}\text { FY 1995 Actual } \\
\text { (12 Agencies) }\end{array}$ & $\$ 1,129$ & $+20.4 \%$ & $\$ 1,019$ & $+10.2 \%$ \\
\hline $\begin{array}{c}\text { FY 1996 Actual } \\
\text { (12 Agencies) }\end{array}$ & $\$ 1,043$ & $-7.6 \%$ & $\$ 949$ & $-6.9 \%$ \\
\hline $\begin{array}{c}\text { FY 1997 President's } \\
\text { Request } \\
\text { (12 Agencies) }\end{array}$ & $\$ 1,040$ & $-0.1 \%$ & $\$ 966$ & $+1.8 \%$ \\
\hline
\end{tabular}

Section 3 of this Plan provides an overview of the HPCC Program. Section 4 contains more detailed definitions of the Program Component Areas, with an emphasis on the overall directions and milestones planned for each PCA. Appendix A provides a detailed look at HPCC Program activities within each agency.

${ }^{4}$ The published FY 1997 President's HPCC Budget also included funding for the Department of Transportation, which is not yet part of the HPCC Program. 


\section{Program Overview}

Through its goals and objectives, the HPCC Program provides the focus for the member agencies' planning, implementation, and management of activities. Through collaborative coordination, the participating agencies seek to leverage each other's activities wherever possible and to minimize redundancy in activities. However, funding for each agency's HPCC activities flows directly to the agency, and each agency has its own mechanisms to select and evaluate projects funded under this Program. Published reports, workshops, meetings, and continuously updated World Wide Web sites are used to distribute the results of HPCC research and to evaluate overall Program progress.

\subsection{Goals and Objectives}

The High Performance Computing and Communications Program goals are to:

Extend U. S. technological leadership in high performance computing and computer communications;

Provide wide dissemination and application of these technologies to speed the pace of innovation and improve the national economic competitiveness, national security, education, health care, and the environment; and

Provide key enabling technologies for the National Information Infrastructure (NII) and demonstrate selected NII applications

These goals are consistent with the goals of the CIC R\&D programs, and will be realized by focusing the HPCC activities within each of the participating Federal agencies and coordinating those activities among the participants. Throughout the life of the Program, many important applications in government, academia, and inclustry have required far greater computing capability than was available at the time, and that remains true today. The HPCC Program will continue to accelerate the development of scalable computing systems that will have the capability (in terms of computational cycles) and capacity (in terms of memory and communication speed) to address more of these critical applications. It will also accelerate development of the supporting technologies, such as file storage systems, computing environments, and network communications required for effective use of these systems. The Administration's vision for a National Information Infrastructure (NII) makes unprecedented demands for network connectivity, capacity, database availability, information management, access security, and ease of use. The HPCC Program will continue to work with industry to create important elements of the technology base needed for a universally accessible NII and will use this technology to develop and demonstrate prototype National Challengeclass applications. All of these activities depend on inventing more cost-effective approaches to developing and maintaining scalable algorithms and software. Active support must be provided for developing a cadre of highlytrained professionals capable of using these modern computing systems and networks.

\subsection{Definition of Program Component Areas}

The HPCC Program is organized into five Program Component Areas (PCAs) that cover a broad spectrum of computing, information, and communications technology R\&D supported by the Federal government. The PCAs are areas of high priority investments by the Federal agencies that participate in the coordinated R\&D programs. These PCAs represent a new structure that evolved from the original components-High Performance Computing Systems (HPCS), National Research and Education Network (NREN), Advanced Software Technology and Algorithms (ASTA), Information Infrastructure Technology and Applications (IITA), and Basic Research and Human Resources (BRHR). These original components were crafted more than five years ago and characterized the new directions that were being explored in high performance computing and communications. Advances in knowledge and the rapid pace of development of new technologies have necessitated new descriptions of the activities funded under this Program. The PCAs are defined, briefly, in the following. More extensive definitions and characteristics are given in section 4 .

\section{High End Computing and Computation (HECC):}

HECC R\&D is focused on continued U.S. leadership in high performance computing and computation. Investments concentrate on leading-edge innovations in hardware and software such as storage and data technologies for highend computing systems, experimentation with novel devices, development of system software technologies, advanced simulation techniques, and fast, efficient algorithms for simulation and modeling. In addition, HECC research supports exploration of advanced computing concepts in quantum, biological, and optical computing at both the hardware and software levels. At the high end, these technologies enable distributed, multidisciplinary 
computation-intensive, scientific and engineering applications. Scalable systems allow effective deployment of these technologies to the workplace, school, and home.

Large Scale Networking Technologies (LSN):

LSN R\&D will assure U.S. technological leadership in communications through R\&D that advances the leading edge of networking technologies and services. This includes advanced network components and technologies for engineering and management of large-scale networks, both for scientific and engineering R\&D and for other purposes. Areas of particular focus include: (1) technologies and services that enable wireless, optical, mobile and wireline communications; (2) networking software that enables information to be disseminated to individuals, multicast to select groups, or broadcast to an entire network; (3) software for efficient development and execution of scalable distributed applications; (4) software components for distributed applications, such as electronic commerce, digital libraries, and health care; and (5) R\&D infrastructure support and testbeds.

High Confidence Systems (HCS):

HCS R\&D will provide users with high levels of security, protection, reliability, and restorability of information services. Such systems are resistant to system failure and malicious penetration or damage and readily adapt or respond to interference. These systems include both physical components, wired and wireless technologies, the data they contain and transmit, and the software that manipulates these data. HCS R\&D focuses on (1) system reliability (such as management of networks under load, failure, or intrusion; emergency response; firewalls; secure enclaves; and formal methods), (2) security and privacy (including personal identification, access control, authentication, encryption and other privacy assurance techniques, public key infrastructures, and trusted agents for secure distributed computing), and (3) testing and evaluation. Key applications include national security, law enforcement, life- and safety-critical requirements, personal privacy, and protection of critical elements of the National Information Infrastructure.

\section{Human Centered Systems (HuCS):}

HuCS R\&D makes computing systems and communications networks more easily accessible to and usable by a wide range of user communities. These communities include scientists and engineers, educators and students, the workforce, and the general public. Technologies enabling such systems include: (1) "knowledge repositories" and "information agents" for managing, analyzing, and presenting massive amounts of multimedia and multi-source information; (2) "collaboratories" that provide access to knowledge repositories and that facilitate knowledge sharing, group authorship, and control of remote instruments; (3) systems that enable multi-modal human system interactions including speech, touch, and gesture recognition and synthesis; and (4) virtual reality environments and their application to fields including scientific research, health care, manufacturing, and training.

\section{Education, Training, and Human Resources(ETHR):}

The focus of ETHR R\&D is on education and training technologies. The goals of this education and training are to produce (1) researchers and students in high performance computing, communications, information technologies, and their application, and (2) a citizenry with the skills to compete and prosper in the 21st century's information age. ETHR includes curriculum development, fellowships, and scholarships for computational, computer, and information sciences and engineering. It includes the application of interdisciplinary research to learning technologies, and R\&D in information-based learning tools, lifelong learning, and distance learning for people in remote locations.

\subsection{HPCC Management, Planning and Organization}

The HPCC Program is implemented as a partnership among Federal agencies, with strong involvement by U.S. academia and industry. Program oversight and budgetary review are provided by the CCIC and its CIC R\&D Subcommittee. The National Coordination Office for Computing, Information, and Communication (NCO) provides a central focus for interagency R\&D activities and coordinates the activities of the HPCC agencies. The NCO also provides an interface to Congress, academia, industry, and the public. John C. Toole, NCO Director and Chair of the CIC R\&D Subcommittee, reports jointly to the Director of Office of Science and Technology Policy and the President's Science Advisor, Dr. John H. Gibbons, and to the Chair of the CCIC, Dr. Anita K. Jones. 


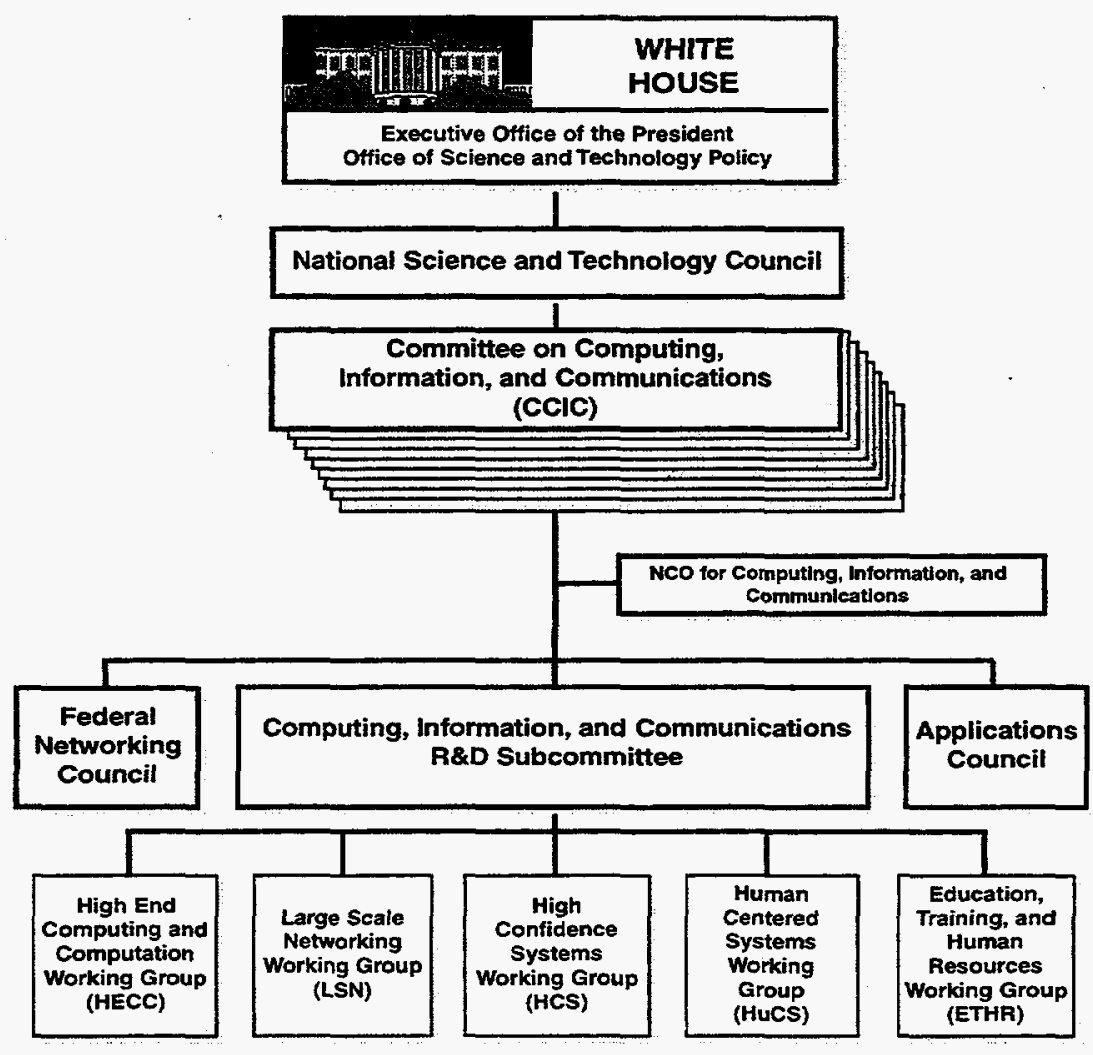

The CIC R\&D Subcommittee meets quarterly to coordinate agency HPCC Programs through information exchanges, common development of interagency programs, and the review of individual agency plans and budgets. The CIC R\&D Subcommittee charters a Working Group for each PCA to coordinate activities in specific areas. Currently active groups include:

- The High End Computing and Computation Working Group, has evolved from the Scientific and Engineering Working Group, and coordinates the development of systems software, software tools, and Grand Challenge applications software. Grand Challenges are computation-intensive fundamental problems in science and engineering with broad economic and scientific impact, in areas such as weather, climate, the environment, manufacturing design, biomedicine, and basic scientific research. This Group is developing agendas for high performance system software technology R\&D and for high performance computing systems research.

- The Human Centered Systems Working Group has evolved from the Information Infrastructure Technologies and Applications Working Group and is developing an agenda for R\&D in human-centered information systems and in distributed collaborative computing.

- Three other Working Groups have been formed, each also associated with a PCA-Large Scale Networking; High Confidence Systems; and Education, Training, and Human Resources.

The Federal Networking Council (FNC) and the Applications Council also report to the CCIC. The FNC is a forum for networking collaboration among Federal organizations operating advanced computer communications networks to meet their research, education, and operational mission goals. The FNC bridges the gap between the advanced networking technologies being developed by R\&D activities and the ultimate acquisition of mature versions of those technologies from the commercial sector. 
The CCIC Applications Council charter is to: (1) provide a forum for exchanging information between information and communications R\&D communities and applications communities that need advanced information and communications technologies, (2) identify information and communications R\&D needed for information technology applications, (3) assess critical information and communications technologies needed by applications (for example, security technologies), and (4) facilitate wider R\&D collaborations on information and communications applications.

\subsection{Selecting Projects for Funding}

To minimize redundancy and encourage adequate funding of all HPCC areas, the CIC R\&D Subcommittee works with the agencies to define complementary areas of responsibility. Within each agency, the mechanisms used to evaluate and fund activities differ. The HPCC agencies support R\&D conducted by agency staff and researchers at universities, in industry, and in national laboratories. Each agency that funds external (university-or industry-based) $R \& D$ activities has a program to review and fund competitive, merit-based awards that are consistent with the agency's mission. Calls for proposals for these grants and contracts may be in the form of Broad Area Announcements (BAAs), Requests for Proposals (RFPs), Cooperative Research and Development Agreements (CRADAs), Cooperative Agreement Notices (CANs), etc. These receive wide distribution, including both electronic means (e.g., electronic bulletin boards and World Wide Web sites on the Internet) and traditional media (e.g., Commerce Business Daily). R\&D projects are usually subjected to the peer review process to aid in selection of the highest quality work and to ensure active participation by the research community.

\subsection{Progress Measures}

The Federal HPCC Program provides the stimulation and coordination essential to accelerate progress in R\&D in high performance computing, communications, and information technology. Success is measured using both quantitative metrics, which characterize the capabilities of the technologies in these areas, and qualitative characteristics, which attempt to capture the impact of using new information technologies in the Federal government, academia, industry, and by the general public.

- Examples of quantitative metrics include peak or sustained computer speed (measured in billions of floating point operations per second, "gigaflops"), data storage capacity (trillions of bytes, "terabytes"), the computational time needed to solve a specific application problem, network communication speed (measured in billions of bits per second, gigabits/s or "Gb/s"), and network deployment (number of HPCC nodes, number of universities connected to the Internet through very high bandwidth network links).

- Examples of qualitative characteristics are greater national security, improved public health and safety, and a cleaner environment.

One method for measuring Program progress is the establishment and subsequent review of yearly milestones described in the next section. This document reports on milestones for three Fiscal Years. The FY 1995 milestones are those that were accomplished by the end of FY 1995 (September 30, 1995). The FY 1996 milestones reported herein are the expected accomplishments for FY 1996, based on Congressional appropriations. FY 1997 milestones describe anticipated accomplishments, assuming Congressional appropriations at the President's requested level. Achievement of these milestones depends on specific program activities that take place within the agencies. The successful completion of these activity-specific milestones across all agencies meets the HPCC Program goals and objectives.

HPCC Program objectives and agencies' project activity milestones are the primary measures of progress when success can be measured quantitatively. When progress cannot obviously be measured quantitatively, qualitative measures are used. One aspect of HPCC research is to identify and use effective measures of progress. Whether quantitative or qualitative, the HPCC Program works with program managers and technical reviewers to assess progress.

\subsection{Reporting Results and Interactions}

At the individual project level, investigators present their results in workshops, conferences, and journal publications. In addition, investigators rely on electronic means (such as the National HPCC Software Exchange) to share and distribute software tools. At the agency level, periodic reports summarize the results of HPCC activities. In addition, HPCC agencies' Web sites provide direct access to the results of their research projects. The NCO has established its own Web site that is linked to all of the agency-level sites. 
In FY 1996, the CIC R\&D Subcommittee and its Working Groups (or their predecessors) sponsored the following outreach conferences and workshops:

- "Computing Systems Briefings" (October, 1995) in which major U.S. vendors gave briefings on technology trends, $R \& D$ needs, and markets and products

- "Electronic Payments Workshop" (November, 1995), which addressed interoperability of multiple electronic payment systems

- A workshop on "Federal Strategies and Programs in the Age of Information" at Supercomputing '95 (December 1995)

- "Petaflop Architecture Workshop, PAWS" (April, 1996)

- "PetaSoft Systems Software Summer Study (PetaSoft '96)" (June 1996)

\subsection{Program Accomplishments}

In 1996 the HPCC Program will complete its fifth year of interagency coordination. Its accomplishments include:

- The R\&D needed to address scaling issues of the Internet from the dozens of users when it was created to several million users in 1996

- Technologies underlying gigabit-speed networks that are a thousand times faster than networks available five years ago. This high transfer rate is required for multimedia communications

- Computing systems architectures, such as high performance scalable parallel systems and networks of workstations, that provide computing speeds up to three orders of magnitude faster than those available five years ago

- Software that makes these communications and computing technologies easy to use for developing scientific, engineering, and business applications

- Technologies that build upon this communications, computing, and software base:

- Scientific visualization

- Virtual reality

- Internet access technologies

- Digital libraries

- Electronic commerce

- Security and privacy technologies

- Grand Challenge and National Challenge applications software that use these computing and communications technologies to yield new knowledge and capabilities never before possible. Some of the areas are:

- The environment-more accurate weather and severe storm forecasts; fundamental understanding of the global climate; improved modeling of air and water resulting in better environmental quality and better management of natural resources; better understanding of the effects of the environment on humans and the effects of humans on the environment; and improved modeling of energy resources

- Design and manufacturing-design of safer and more fuel-efficient engines for cars, trucks, and airplanes; more energy-efficient homes, offices, and factories; new products that are lighter, stronger, safer, and cheaper to manufacture and use and that have faster time to market

- Biomedicine and health care-improved understanding of the human body and the effects of disease, injury, and surgical intervention; better understanding of biological molecules; a more effective and more efficient health care system; improved clinical decision making; and more accurate, uniform, and retrievable patient care data

- National security and national defense-improved civil and defense infrastructure including U.S. transportation, energy, and communications systems; and protection of critical information systems against attack and in times of emergency

- Basic and applied research - better understanding of the physical world and the universe through simulation of events too large, too small, too fast, too slow, too dangerous, or too far away to observe directly

- Education-better educational, vocational, and cultural materials available to all citizens regardless of age, geographic location, or ability

- Government-supported information, including scientific data from HPCC agencies and Federal R\&D program descriptions, is freely and easily available to all citizens

- The education and training of new generations of developers and users of these technologies (ranging from researchers in science and engineering to students of all ages) 
Previous years accomplishments are also documented in:

- "Advancing the Frontiers of Information Technology," a Supplement to the President's FY 1997 Budget

- "High Performance Computing and Communications: Foundation for America's Information Future," a Supplement to the President's FY 1996 Budget

- "Federal HPCC FY 1996 Implementation Plan," which details HPCC plans and budget crosscut

- A four-page flier that documents five years of HPCC accomplishments

These publications are available in print from the NCO and at the Program's Web site www.hpcc.gov.

\subsection{Program Oversight}

The President has selected a High Performance Computing Advisory Committee of non-Federal members, including representatives of the research, education, library communities, network providers, and industry, who are specially qualified to provide advice and information on high performance computing and communications. While this Advisory Committee is now being appointed, representatives from academia, industry, and professional associations have conferred with and advised the CIC R\&D Subcommittee in several public sessions.

\subsection{Agency Program Oversight}

Detailed program reviews are also carried out by individual agencies via their management review structures, including official advisory committees. This section highlights recent and current oversight and review activities and describes existing review mechanisms within each of the agencies.

The Defense Advanced Research Projects Agency (DARPA) reviews its HPCC efforts at many different levels to ensure consistent program evaluation in a dynamic $R \& D$ environment. Projects produce reports on a regular basis that are reviewed by program managers. An annual process of updating accomplishments, milestones, and project plans is tied to the incremental funding process. Site visits, project meetings, principal investigator meetings, and regular interactions using the Internet as an information infrastructure enable DARPA staff to fulfill their program management responsibilities. In addition, contracting details are handled by contracting agents that work with DARPA as part of the program management process. Office directors and program managers develop plans and milestones that are approved by senior management during the planning and budget cycle. New programs and ideas are proposed during this process. In conjunction with yearly funding decisions by DARPA and DoD, senior DARPA technical management critically review program areas, plans, and accomplishments. Guidance is provided to reflect programmatic, technical, and funding directions. At the DoD level, programs are described through a formal process that requires agency, DARPA Comptroller, DoD Comptroller, senior DARPA management, and senior DoD approvals. Once approved, these descriptions become part of the Defense budget submitted to Congress for approval. In addition to other internal Federal HPCC reviews, there are Congressional briefings, HPCC agency crosscuts, technical working groups, DoD advisory panels, and several National Academy of Science studies that contribute to the planning process.

The National Science Foundation (NSF) HPCC program long-term goals and objectives consist of numerous processes and mechanisms that contribute to the definition and review of the supported projects. In addition, more specific goals and objectives are established for each of the activities within the program. The National Science Board, panels and committees commissioned to study and recommend program activities, and external advisory committees contribute primarily to the more general, long-term goals. The establishment of goals, objectives, implementation mechanisms, evaluative measures, and the broader value of specific activities is accomplished through such means as external peer review (mail reviews, panel reviews, and site visits), workshops for developing research agendas, program committees of visitors, technical oversight teams, ongoing site visits by program staff and outside experts, program officer review of final project reports, and the bodies of opinion held by the community of researchers themselves. Evaluation of progress is integrated into the management process as appropriate to each class of HPCC activities. Examples are:

- Federal Networking Council Advisory Committee for the Internet

- Directorate Advisory Committees

- Committee of Visitors for each research program 
The Department of Energy (DOE) HPCC program focuses on basic mathematics and computational research and on developing and delivering technology for use by other scientists and engineers in DOE and associated U.S. academia and industry. Performance evaluation is an integral part of these programs. Because of this focus, external review by prospective users of the technology is a critical component of measuring performance. In previous years this has been most explicitly present in the use of committees of users such as the ESNet (Energy Sciences Network) Steering Committee and the ER (Energy Research) Supercomputer Users Group to evaluate the effectiveness of the access and networking programs. Many of the education programs established under the DOE HPCC program have evaluation built in. In software technology, employing prospective users of technologies as reviewers has ensured that the technology developed is that required by users. DOE further formalized these procedures to include program-wide reviews of the basic technology components of the program by significant prospective users of those technologies. These include reviews of effectiveness in all categories as well as specific numerical targets.

The National Aeronautics and Space Administration (NASA) HPCC program evaluations take place at several levels. At the agency level, the NASA Advisory Council (NAC) has established the Ad Hoc Task Force on Supercomputing, which completed a review and report on the NASA HPCC program. It is expected that other such bodies will be permanently chartered under the Aeronautics Advisory Committee to advise the NASA HPCC program. Within the program, annual comprehensive reviews are conducted for each of the projects. In addition to appropriate NASA personnel, representatives from other Federal agencies, academia, and industry may be invited to participate. Annual reviews of program progress and plans will also be conducted by the NASA HPCC Working Group. In addition, quarterly reviews of the NASA HPCC program are conducted by program managers, the associate administrator of aeronautics, and the director of the NASA HPCC Office.

The National Institutes of Health (NIH) HPCC program goals are enhancements of existing NIH program missions to support biomedical science and expand biomedical knowledge. Program objectives are developed by Institute Directors, advisory bodies, and senior program staff, and are peer reviewed for determination of merit. Each of the participating NIH components has one or more standing external advisory committees that review new and existing programs. These include the National Library of Medicine (NLM) Board of Regents, NLM Board of Scientific Counselors, National Center for Research Resources (NCRR) Advisory Council, NCRR Biomedical Research Technology Review Committee, the National Cancer Advisory Board, the National Cancer Institute Division of Cancer Biology and Diagnosis Board of Scientific Counselors, and the Division of Computer Research and Technology Advisory Council. The final decision regarding individual HPCC programs within each of the participating Institutes rests with the Director of that Institute. Within each of the participating NIFI Institutes, mechanisms exist to ensure objective evaluation of progress and results and identification of possible new activities.

The National Security Agency (NSA) reviews its HPCC support efforts on a yearly basis in several separate reviews. A steering group composed of senior managers from the technical components provides high level guidance prior to the formal budget process. The steering group receives individual project assessments from the project managers and determines whether any major shifts or changes are needed to the NSA program. Senior management has the flexibility to sponsor HPCC efforts in several budget reviews. Individual projects are proposed and budgeted within the technical components, and are constantly evaluated by the project managers. Monthly status reports are evaluated and meetings held with the project staff to ensure that the correct focus is maintained. During the budget review cycle, the projects are evaluated and terminated or retained based on their performance, importance relative to other initiatives, and priority based on the steering group guidance. New projects can be proposed by the technical components each year during the NSA Technology Program review process.

The National Institute of Standards and Technology (NIST) has the National Academy of Science review annually all the programs and activities of each operating unit, as part of its normal operation. Assessment includes relevance, performance measures, and achievements. Consequently, programs and activities that are a part of the Federal HPCC Program are subject to review and comments of an external panel of experts from academia and industry. A written report is presented to the Director of NIST, the Administration, and Congress. Selection of individual projects and subsequent progress reviews are conducted by the program manager. These are reviewed by an inter-operating unit panel composed of senior managers for relevance to agency mission and the Program, and for continued acceptable performance.

The National Oceanic and Atmospheric Administration (NOAA) HPCC Program supports and enhances NOAA programs in environmental prediction and stewardship. Comprehensive NOAA science reviews of these programs are held periodically. Quarterly reviews of NOAA HPCC program progress are conducted as an integral part of 
NOAA-wide quarterly reviews by the NOAA Administrator and other NOAA senior line and program managers. Overall NOAA HPCC program goals and plans are reviewed annually as part of the NOAA strategic planning process.

The Environmental Protection Agency (EPA) HPCC program is focused on incorporating advances in computing and communications technology into critical environmental assessment applications and transferring those advanced tools to key state, Federal, and industrial users. EPA senior management officials review the EPA HPCC program annually to assess its relevance to the agency mission and program achievements. Agency guidelines require an external peer review of the EPA HPCC program every two years. The external review panel is composed of representatives from other Federal agencies, academia, and industry. Within the program, each major project is reviewed at least twice a year to evaluate progress toward the program objectives.

The Department of Education (ED) technology initiatives are focused on expanding the use of advanced computer and telecommunication technologies to help students achieve challenging academic standards. ED has awarded 5year grants to 43 consortia of local school systems, colleges, universities, and private businesses to develop creative ways to use technology for learning. These and other R\&D efforts also focus on the needs of handicapped learners, migrant students, and other populations with special needs, as well as to strengthen the links between home and school, teachers and their colleagues, and students over wide geographic areas and among heretofore diverse communities of learners.

The Agency for Health Care Policy Research (AHCPR) HPCC program focuses on health care technology applications of computer based patient records, computerized clinical decision support systems, and patient care data standards. These HPCC activities are predominantly technology applications support for promoting the development and evaluation of systems to foster their economic and medical feasibility.

The Department of Veterans Affairs (VA) HPCC program focuses on data capture, information systems, local area and wide area networks connecting a significant number of its member facilities, which includes 171 VA medical centers, 362 outpatient clinics, 129 nursing homes, and 35 domiciliaries.

\subsection{HPCC Planning Beyond FY 1997}

The CIC R\&D Subcommittee will continue to seek input and comment from academia, industry, other segments of the government, and the public, through a wide range of interchanges.

The assessment of options and benefits that derive from continued efforts to stimulate computing and communications technologies will be investigated by sponsoring a variety of workshops with technical experts and potential users of these technologies. In FY 1996, the numerous HPCC-related workshops listed in section 3.6 have been a part of that process. As an additional part of this process, the Subcommittee will actively seek comments on this Implementation Plan.

Computing and communications are ongoing strategic enabling technologies. The HPCC Program provides additional stimulation to accelerate progress in developing these technologies and in benefiting from their use. Through broad input and extensive discussions both within and outside the Federal government, the CIC R\&D Subcommittee expects to develop a long-term R\&D plan as well as detailed implementation proposals that will evolve naturally into a budget planning process. 


\section{HPCC FY 1997 Budget Overview by Program Component Areas}

This section presents an HPCC Program overview by agency by year by PCA. The presentation begins with the proposed agency-level funding on which the planned activities are based. The following summary information is presented for each PCA:

- Description of the types of activities included

- Status report describing changes from FY 1996 and their possible future impact

- List of milestones expected to be accomplished in FY 1997

- Table of the agency activities supporting the area

\subsection{Budget Planning Assumptions by PCA}

Table 1 summarizes the financial planning information prepared by each HPCC agency for this Implementation Plan. The "FY 95 Actual" column refers to the actual funds appropriated for that year. The "FY 96 Pres." and "FY 97 Pres." columns refer to the funds requested by the President in his budget request to Congress for those two years. The "FY 96 Est." column estimates the funds each agency will have been authorized to spend on HPCC as a result of Congressional appropriations, effective as this Implementation Plan goes to press. The last five columns break down the FY 1997 Presidential Request into the planned spending level for each of the five PCAs. These breakdowns are subjective, since an activity may span several PCAs.

In Appendix A, the FY 1995 milestones identify actual accomplishments, and the FY 1996 milestones assume the FY 1996 estimated funding level for each agency. The FY 1997 milestones assume the FY 1997 Presidential Request funding level in each agency. All discussions of program status also assume these numbers.

Table 1A provides a comparison by PCA of funding in FY 1996 with FY 1997. Both tables are obtained from a database that is constantly being updated as new information is obtained from the agencies about Congressional action, agency funding redirections, etc. 
Table 1: HPCC FY 1997 IP Budget Summary

\begin{tabular}{|c|c|c|c|c|c|c|c|c|c|}
\hline \multirow[b]{2}{*}{ Agency } & \multicolumn{4}{|c|}{ Budget (BA, \$ M) } & \multicolumn{5}{|c|}{ HPCC PCAs by 1997 President's Request } \\
\hline & $\begin{array}{l}\text { FY 95 } \\
\text { Actual } \\
\end{array}$ & $\begin{array}{c}\text { FY } 96 \\
\text { Pres. } \\
\end{array}$ & $\begin{array}{l}\text { FY } 96 \\
\text { Est. }\end{array}$ & $\begin{array}{l}\text { FY } 97 \\
\text { Rqst. }\end{array}$ & HECC & LSN & HCS & HuCS & ETHR \\
\hline DARPA & 335.06 & 353.61 & 302.41 & 325.52 & 75.05 & 115.93 & 10.00 & 117.03 & 7.51 \\
\hline NSF & 321.73 & 313.64 & 291.10 & 279.51 & 129.17 & 72.26 & 1.21 & 57.76 & 19.11 \\
\hline DOE & 122.35 & 109.69 & 109.69 & 124.56 & 93.29 & 14.79 & & 12.98 & 3.50 \\
\hline NASA & 131.40 & 136.30 & 126.60 & 110.10 & 87.20 & 15.30 & 1.00 & 3.40 & 3.20 \\
\hline $\mathrm{NIH}$ & 67.35 & 77.91 & 79.74 & 86.21 & 23.40 & 22.90 & 4.94 & 29.59 & 5.38 \\
\hline NSA & 39.93 & 40.03 & 40.03 & 36.73 & 25.93 & 3.50 & 7.30 & & \\
\hline NIST & 23.51 & 34.10 & 23.51 & 23.51 & 5.79 & 2.96 & 5.20 & 9.56 & \\
\hline $\mathrm{ED}$ & 27.98 & 16.95 & 28.93 & 18.01 & & & & 11.40 & 6.61 \\
\hline VA & 37.55 & 36.00 & 21.93 & 14.55 & 1.00 & 9.45 & 2.30 & 1.80 & \\
\hline NOAA & 5.50 & 15.40 & 6.50 & 9.50 & 6.30 & 2.70 & & 0.50 & \\
\hline EPA & 12.43 & 12.00 & 9.38 & 7.18 & 6.58 & & & 0.60 & \\
\hline AHCPR & 4.10 & 8.40 & 3.20 & 4.20 & & & & 4.20 & \\
\hline Totals & 1128.89 & 1154.03 & 1043.02 & 1039.58 & 453.71 & 259.79 & 31.95 & 248.82 & 45.31 \\
\hline
\end{tabular}




\begin{tabular}{|c|c|c|c|c|r|r|r|r|r|r|}
\hline \multicolumn{1}{|c|}{ HPCC Program Component Areas by 1997 President's Request } & \multicolumn{3}{|c|}{ HPCC Program Component Areas by 1996 Expected } \\
\hline AGENCY & HECC & LSN & HCS & HuCS & ETHR & HECC & LSN & HCS & HuCS & ETHR \\
\hline DARPA & 75.05 & 115.93 & 10.00 & 117.03 & 7.51 & 77.96 & 96.04 & 10.00 & 112.17 & 6.24 \\
\hline NSF & 129.17 & 72.26 & 1.21 & 57.76 & 19.11 & 140.32 & 104.47 & & 15.28 & 31.03 \\
\hline DOE & 93.29 & 14.79 & & 12.98 & 3.50 & 84.49 & 12.64 & & 8.56 & 4.00 \\
\hline NASA & 87.20 & 15.30 & 1.00 & 3.40 & 3.20 & 75.55 & 27.45 & & & 23.60 \\
\hline NIH & 23.40 & 22.90 & 4.94 & 29.59 & 5.38 & 22.40 & 21.51 & 4.38 & 24.34 & 7.11 \\
\hline NSA & 25.93 & 3.50 & 7.30 & & & 29.48 & 3.00 & 7.40 & & 0.15 \\
\hline NIST & 5.79 & 2.96 & 5.20 & 9.56 & & 5.59 & 2.20 & 5.36 & 10.36 & \\
\hline ED & & & & 11.40 & 6.61 & & & & 11.40 & 17.53 \\
\hline VA & 1.00 & 9.45 & 2.30 & 1.80 & & 3.00 & 14.13 & 2.90 & 1.90 & \\
\hline NOAA & 6.30 & 2.70 & & 0.50 & & 3.30 & 2.70 & & 0.50 & \\
\hline EPA & 6.58 & & & 0.60 & & 8.70 & & & 0.60 & 0.08 \\
\hline AHCPR & & & & 4.20 & & & & & 3.20 & \\
\hline Totals & $\mathbf{4 5 3 . 7 1}$ & $\mathbf{2 5 9 . 7 9}$ & $\mathbf{3 1 . 9 5}$ & $\mathbf{2 4 8 . 8 2}$ & $\mathbf{4 5 . 3 1}$ & $\mathbf{4 5 0 . 7 9}$ & $\mathbf{2 8 4 . 1 4}$ & $\mathbf{3 0 . 0 4}$ & $\mathbf{1 8 8 . 3 1}$ & $\mathbf{8 9 . 7 4}$ \\
\hline
\end{tabular}




\subsection{High End Computing and Computation (HECC)}

\subsubsection{HECC Definition}

As defined in the FY 1997 Blue Book:

"HECC R\&D is focused on continued U.S. leadership in high performance computing and computation. Investments concentrate on leading-edge innovations in hardware and software such as storage and data technologies for highend computing systems, experimentation with novel devices, development of system software technologies, advanced simulation techniques, and fast, efficient algorithms for simulation and modeling. In addition, HECC research supports exploration of advanced computing concepts in quantum, biological, and optical computing at both the hardware and software levels. At the high end, these technologies enable distributed, multidisciplinary computationintensive, scientific and engineering applications. Scalable systems allow effective deployment of these technologies to the workplace, school, and home."

HECC R\&D investments are made in four key science and technologies areas: hardware, software, systems architecture, and applications R\&D. Hardware R\&D includes low latency, high bandwidth memory technologies, innovative mass storage concepts, and fast, efficient logic devices employing new superconducting, optical, biological and semiconducting materials technology. Software R\&D includes innovative compilers, debuggers, and fast, efficient algorithms for simulation and modeling. Systems architecture R\&D is focused on scalable and distributed operating systems. The applications area R\&D includes support for centers for supercomputing and advanced experimental computing systems architectures. $R \& D$ investments in all four areas are necessary to enable development of the distributed, multidisciplinary computation-intensive applications mandated by future national and international scientific, military, and commercial markets.

Many of the HPCC agencies support scientific mission-driven applications called "Grand Challenge" projects. These projects span the spectrum of scientific problems from cosmology to quantum chromodynamics, to applications in global climate modeling and protein folding. These projects involve available high end computing systems, such as parallel vector processors (PVPs), symmetric multiprocessors (SMPs), and massively parallel processors (MPPs). Grand Challenge projects depend on high performance compatible hardware and software, such as memories, processors, mass storage, operating systems, program development and data management tools, and network protocols.

Scalability is a very important consideration in HECC R\&D. How well a mid-range computing system scales to a high end computing system is of paramount interest. The challenge is to find a scaling process, such that marketdriven mid-range computing systems will scale in performance to the higher end systems in a cost-effective way. The same mid-range program development and management models must be viable for the large end systems necessary to meet the computational requirements of advanced problems associated with the Grand Challenge projects. For clusters of SMPs to be effective in high end computing, the technologies and parameters associated with software, storage, computing, and networking must scale in a fashion that minimizes costs and maximizes computing power.

Very large scale parallel computing will eventually garner wide scale acceptance outside the scientific and engineering communities by successfully addressing the following challenges:

- Developers of software tools and programming environments must keep pace with developers of advanced computational hardware

- An acceptable unified reference model for programming large-scale applications such as the Grand and postGrand Challenge projects must be developed

- Data management tools for large, diverse data sets must be developed

The consolidation and focus of research on the shared-memory programming model, as commercially delivered in medium scale (fewer than 100 processors) SMPs, is one evolutionary method that addresses the first challenge. Research should be focused on developing new hardware to overcome latency and transportable software technologies that cost-effectively scale the SMP model to significantly larger numbers of processors across diverse hardware architectures. The primary benefit will be to stabilize the underlying programming model for the development of software tools and applications, enabling the software-developer community to more easily support high performance systems. 
An important requirement for addressing the second challenge is a unified programming model that can be adopted consistently across a wide range of architectures. The diversity of parallel architectures and associated programming models has expanded our understanding of scalable computing, indicating the need for the generation of portable software development tools, execution environments, and scalable applications.

Large scale data management presents a third primary challenge to technological advancement in high performance parallel computing; the development of the storage hierarchy hardware technology, from petabyte tape robots to large, fast on-chip memories. Effective data management demands systems engineering and integration of components supplied by many vendors. Existing hardware components must be integrated into very large dataintensive systems. Experience has shown that data management testbeds must be tested at full scale.

The Supercomputer Centers and High Performance Computation Research Centers provide some of the largest systems of scalable SMPs available today for addressing large-scale applications. Researchers using these systems are currently faced with the lack of versatile, portable programming environment tools needed to implement their applications. Support for operating systems software and program development environments is an important thrust of the HECC R\&D activity.

High end computing also requires R\&D focused on logic devices that will have delay-power products orders of magnitude less than the delay-power products of current commercial devices. This can be accomplished by a revolutionary redesign of the logic circuits and migration to a new materials technology. Some promising concepts in the research stage include logic circuits based on semiconducting materials such as the III-V binary alloys. R\&D must be conducted on other novel materials, such as low and high temperature superconductors used for logic circuits and memories. Other promising concepts are on- and off-chip interconnections based on guided optics that employ wave division multiplexing, massive holographic optical memory devices, and other mass storage concepts. Basic research must be done on innovative logic devices based on nanotechnology and biological materials that may exploit the information contained in large molecules such as deoxyribonucleic acid (DNA).

\subsubsection{HECC Status}

Funds to support activities in High End Computing and Computation for FY 1997 total approximately \$4.54 million, compared to the FY 1996 Estimated budget of $\$ 451$ million, an increase of $0.6 \%$.

Effective in FY 1997, NSF has discontinued reporting as HPCC Program activities approximately $\$ 9$ million in the Supercomputer Centers' budgets and approximately $\$ 16$ million in activities in theoretical computer science-these actions were recommended in the National Research Council's Computer Science and Telecommunications Board report reviewing the HPCC Program. However, new programs in Computing Systems make up for this decrease, emphasizing fundamental research in the design and implementation of computing systems. NSF will initiate the Partnerships for Advanced Computational Infrastructure (PACI) to provide access to high performance computing for the academic research community at a level of one to two orders of magnitude greater than that typically available at a major research university. NSF will begin a new, interdisciplinary initiative to apply massively parallel computing using biological materials such as DNA. NSF plans to support research to determine how formal, automated design techniques can be used to enable rapid advances in design for nanomanufacturing.

In DOE, new activities, such as the new Advanced Computational Testing and Simulation (ACTS) project, are designed to provide simulations of difficult (even impossible) or dangerous experimental phenomena. DOE will recompete both the HPCRC and the Grand Challenges projects for a second phase. They will begin development of software tools that are designed around the ACTS Toolkit interface definitions and integrate most critical existing software tools into the ACTS framework. DOE plans to deliver high performance data management and archival storage software to users of DOE high performance computer centers by integrating file systems of supercomputers in access centers using the High Performance Storage System. DOE will also begin development of ACTS-enabled applications development in compressible fluid dynamics, combustion, environmental chemistry, and materials sciences. This effort is coordinated with parallel development in DOE Defense Programs of ACT.S-enabled applications in weapons hydrodynamics and materials characterization and aging.

DARPA is developing new operating systems capabilities that include object management, high assurance, and support for real time distributed applications. They are continuing their high performance language and compiler research and developing advanced software environments. DARPA will provide scalable versions of widely used 
commercial engineering software, including MSC NASTRAN, leveraging scalable software library technology available to the defense community.

NASA plans to support long-term HECC projects in high performance computing systems research and high performance systems software and technology, in coordination with DARPA, NSF, and DOE. NASA's Earth and Space Sciences (ESS) scalable testbed will support the achievement of 50 gigaFLOPS sustained on ESS investigator codes. NASA is also interested in an end-to-end reduction in cost and time to solution for aerospace design applications on distributed workstation clusters at $25 \%$ of the capital cost of a 1994 comparison base. In support of this effort, NASA will develop commercially available systems software that provides high availability and portability demonstrated in a large-scale production environment.

NIH will continue use of high performance computer resources accessible through high speed networks to generate biomedical images, support structure-based drug design, and perform large-scale computer simulations to elucidate the structure and function of biological macromolecules including assemblies of macromolecules such as cell membranes. The intent of the NIH program is to expand activities in high performance computing applications to determine and predict the structure of proteins, apply computational chemistry to understanding of drug interactions with enzymes and nucleic acids as a basis for discovery of new drugs, and develop new positron emission tomography imaging methods.

NSA will begin a new initiative in materials, devices, and optoelectronics for very high communication rates and computing. They will continue with the design of a uniform memory access supercomputer without a backplane, seeking to improve processor-to-memory latency and bandwidth by an order of magnitude. They will continue development of a superconducting crossbar switch and associated electronics.

NIST will stabilize and release a scalable parallel multigrid solver for unstructured adaptive grids and demonstrate high-performance object-oriented libraries for sparse linear algebra on challenging applications.

NOAA plans to implement its ETA regional model ("ETA" refers to the type of vertical coordinate used in the model) on one of its massively parallel systems. They will evaluate the model's performance at several grid resolutions and assess its potential for use in operational forecasting and for updating real-time experimental forecast models to support nesting: NOAA's National Centers for Environmental Prediction (formerly the National Meteorological Center) plan to use scalable high performance computing systems to enable future improvements in operational weather forecasting and to initiate algorithm development on scalable systems with the goal of achieving 5-10 kilometer resolution in mesoscale atmospheric models.

\subsubsection{HECC 1997 Expected Milestones}

- Demonstrate 100 gigaops/cu. ft. militarized high performance computing systems

- High performance CAD demonstration of $64 \mathrm{Mb} / \mathrm{s}$ CMOS wireless transceiver

- Demonstrate advanced object management systems integrated with operating systems and applications to achieve efficient use of memory while enhancing execution speed

- Demonstrate high-level, portable parallel test generation system with fully-integrated, parameterized, constraint-driven design libraries, including portable software templates for teraFLOPS scalable testbeds

- Demonstrate initial multisite collaborative design research environment for integrated circuit process simulation and remote experimentation over the Internet, using advanced software environment that supports composition tools for composing software, integration, and software development and testing using animation techniques

- Demonstrate optimizing compilers with 5-10 times runtime performance improvement through partial compilation and late optimization during program execution

- Demonstrate real-time distributed operating systems for embeddable high performance computing systems

- Demonstrate integrating testbed architecture incorporating advanced distributed simulation, advanced distributed collaboration, advanced communications and control, and advanced human computer interfaces

- Demonstrate initial capabilities of intelligent information services architecture with multiple mechanisms for describing resource capabilities and with a uniform interface to hybrid search methods for resource retrieval

- Demonstrate systems for coordinating sets of workstations as a single computing system and demonstrate scalability from distributed workstation clusters to teraFLOPS supercomputers on the identical technology base

- Demonstrate end-to-end reduction in cost by a factor of four and in time to solution by a factor of five for aerospace design applications on heterogeneous workstation clusters 


\begin{tabular}{|c|c|c|c|c|}
\hline $\begin{array}{|cc|}\text { FY } 97 \\
\text { Total Pres } \\
\text { (BA } \quad \$ M) \\
\end{array}$ & $\begin{array}{c}\text { FY } 96 \\
\text { Total Est } \\
(\mathrm{BA} \quad \$ \mathrm{M}) \\
\end{array}$ & $\begin{array}{l}\text { Agency / Program Activity } \\
\text { High End Computing and Computation }\end{array}$ & $\begin{array}{c}\text { FY } 97 \\
\text { Pres. } \\
\text { (BA \$M) }\end{array}$ & $\begin{array}{c}\text { FY } 96 \\
\text { Est } \\
\text { (BA \$M) }\end{array}$ \\
\hline \multirow[t]{6}{*}{75.05} & 77.96 & DARPA & & \\
\hline & & Scalable Systems and Software & 27.17 & 32.63 \\
\hline & & Microsystems & 14.29 & 16.25 \\
\hline & & System Environments & $\mathbf{1 5 . 5 0}$ & 18.28 \\
\hline & & Defense Technology Integration and Infrastructure & 14.00 & 8.20 \\
\hline & & Information Sciences & 4.09 & 2.60 \\
\hline \multirow[t]{11}{*}{129.17} & 140.32 & NSF & & \\
\hline & & Supercomputer Centers & 57.73 & 69.36 \\
\hline & & Computing Systems & 45.95 & \\
\hline & & Applications & 25.49 & \\
\hline & & Research Centers & & 6.30 \\
\hline & & Research Infrastructure & & 8.64 \\
\hline & & Grand Challenge Applications Groups & & 7.32 \\
\hline & & Computing Systems and Components & & 17.20 \\
\hline & & Software Systems and Algorithms & & 26.66 \\
\hline & & Engineering (non-NC/GC) & & 1.65 \\
\hline & & Geosciences (non-NC/GC) & & 3.19 \\
\hline \multirow[t]{6}{*}{93.29} & 84.49 & DOE & & \\
\hline & & Advanced Computational Testing and Simulation Research & 32.90 & 31.81 \\
\hline & & Grand Challenge Applications & $\mathbf{1 0 . 0 0}$ & 10.00 \\
\hline & & DOE2000 ACTS & 5.00 & \\
\hline & & National Energy Research Scientific Computing Center & 29.76 & 30.30 \\
\hline & & High Performance Computiting Resource Providers & .15 .63 & 12.39 \\
\hline
\end{tabular}

Table 2: High End Computing and Computation Program Activity Summary for FY 1997 Presidential Budget 


\begin{tabular}{|c|c|c|c|c|}
\hline $\begin{array}{c}\text { FY } 97 \\
\text { Total Pres } \\
\text { (BA } \$ \text { \$M) } \\
\end{array}$ & $\begin{array}{c}\text { FY } 96 \\
\text { Total Est } \\
\text { (BA } \$ M \text { ) }\end{array}$ & $\begin{array}{l}\text { Agency / Program Activity } \\
\text { High End Computing and Computation }\end{array}$ & $\begin{array}{c}\text { FY } 97 \\
\text { Pres. } \\
\text { (BA \$M) }\end{array}$ & $\begin{array}{c}\text { FY } 96 \\
\text { Est } \\
(\mathrm{BA} \$ \mathrm{M})\end{array}$ \\
\hline \multirow[t]{6}{*}{87.20} & 75.55 & NASA & & \\
\hline & & Testbeds & 15.39 & 12.91 \\
\hline & & Grand Challenge Support & 56.75 & 52.62 \\
\hline & & Systems Software & 7.56 & 10.02 \\
\hline & & Information Infrastructure Technology & 3.50 & \\
\hline & & Information Infrastructure Applications & 4.00 & \\
\hline \multirow[t]{8}{*}{23.40} & 22.40 & NIH & & \\
\hline & & NCRR Biomolecular Computing & 6.30 & 5.80 \\
\hline & & NCRR Software Tools for Receptor-Based Drug Design & 2.20 & 2.20 \\
\hline & & NCRR Modeling/Simulation & 4.50 & 4.50 \\
\hline & & DCRT High Performance Biomedical Computing Program & 6.10 & 6.10 \\
\hline & & NCI Frederick Biomedical Supercomputing Center & 3.60 & 3.60 \\
\hline & & NCl High Speed Networking and Distributed Conferencing & 0.20 & 0.20 \\
\hline & & NIGMS & 0.50 & \\
\hline \multirow[t]{3}{*}{25.93} & 29.48 & NSA & & \\
\hline & & Supercomputing Research & 23.43 & 27.48 \\
\hline & & Superconducting Research & 2.50 & 2.00 \\
\hline \multirow[t]{4}{*}{5.79} & 5.59 & NIST & & \\
\hline & & Development and Dissemination of Scientific Software for HPCS & 2.77 & 2.37 \\
\hline & & Infrastructure for Information Technology & 1.22 & 1.22 \\
\hline & & Systems Integration for Manufacturing Applications & 1.80 & 2.00 \\
\hline \multirow[t]{2}{*}{1.00} & 3.00 & VA & & \\
\hline & & VA Hybrid Open Systems Technology (VA HOST) & 1.00 & 3.00 \\
\hline
\end{tabular}

N

Table 2: High End Computing and Computation

Program Activity Summary for FY 1997 Presidential Budget

Page 17 


\begin{tabular}{|c|c|c|c|c|}
\hline $\begin{array}{c}\text { FY } 97 \\
\text { Total Pres } \\
(\mathrm{BA} \$ \mathrm{M})\end{array}$ & 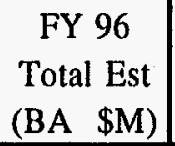 & $\begin{array}{l}\text { Agency / Program Activity } \\
\text { High End Computing and Computation }\end{array}$ & $\begin{array}{c}\text { FY } 97 \\
\text { Pres. } \\
\text { (BA \$M) }\end{array}$ & $\begin{array}{c}\text { FY 96 } \\
\text { Est } \\
\text { (BA \$M) }\end{array}$ \\
\hline \multirow[t]{2}{*}{6.30} & 3.30 & NOAA & & \\
\hline & & Advanced Computation & 6.30 & 3.30 \\
\hline \multirow[t]{3}{*}{6.58} & 8.70 & EPA & & \\
\hline & & Environmental Modeling & 3.45 & 5.53 \\
\hline & & Computational Techniques & 3.13 & 3.17 \\
\hline 453.71 & 450.79 & HECC FY 1997 Total & 453.71 & 450.79 \\
\hline
\end{tabular}




\subsection{Large Scale Networking (LSN)}

\subsubsection{LSN Definition}

\section{As defined in the FY 1997 Blue Book:}

"LSN R\&D will assure U.S. technological leadership in communications through $R \& D$ that advances the leading edge of networking technologies and services. This includes advanced network components and technologies for engineering and management of large-scale networks, both for scientific and engineering $R \& D$ and for other purposes. Areas of particular focus include (1) technologies and services that enable wireless, optical, mobile, and wireline communications; (2) networking software that enables information to be disseminated to individuals, multicast to select groups, or broadcast to an entire network; (3) software for efficient development and execution of scalable distributed applications; (4) software components for distributed applications, such as electronic commerce, digital libraries, and health care; and (5) software for infrastructure support and testbeds."

Existing network transport protocols are very successful in enabling data transport across heterogeneous systems. In addition to this basic level of service, interoperability requires higher level functions that include partitioning, placement, migration of applications into distributed components of communications, computation, and storage, and the allocation of system resources to their execution. As servers and their caches of information move through the network to different host systems and adapt under different service loads, applications must be able to discover their location. Applications will need to locate and access global libraries of multimedia objects. As the various national information infrastructures aggregate into a true Global Information Infrastructure, more reliable mechanisms for cooperative authentication, resource allocation, and charging are needed.

Components for high-level object services must be designed to be highly adaptive and composable. Reliable mechanisms are needed, especially in a complex inter-networked environment, to locate and register object services and to certify object and service functionality. More capable scripting and agent services are needed to describe and execute arbitrary tasks. Competent high-level support services are needed to create, publish, record, disseminate, and protect digital objects, such as multimedia documents. To insure that objects interoperate, new technologies such as mediators and wrappers are needed, as well as frameworks that support interoperability through common object protocols, interfaces, and adapters. This research includes the development and implementation of Asynchronous Transfer Mode (ATM) and Wavelength Division Multiplexing (WDM) technologies.

In order to rapidly and accurately analyze varied information, technologies must be developed to enable the rapid correlation of collections of multimedia objects physically distributed across different repositories, which may be geographically dispersed. This requires the development of the next generation of object (data and computation) retrieval, filtering, indexing, and digital library technologies. These technologies will enable personal adaptive information spaces critical for:

- scientific analysis environments

- remote instruments

- remote computational models of physical phenomena

- complex system and product design and analysis environments

- business trend analysis and filtering

Information brokering refers to the storage of information, whereas analysis deals with the retrieval of information. Technology for information brokering must be developed to make it significantly easier to author new kinds of information objects and to charge for the access and use of such objects. This includes tools for group authoring, annotation of objects, and collaboration on evolving objects. An important form of brokering service will be recording and sharing annotated navigation paths through the network, in order to provide value-added summaries of the materials available.

\subsubsection{LSN Status}

Funds to support activities in Large Scale Networking for FY 1997 total approximately $\$ 260$ million, compared to the FY 1996 Estimated budget of $\$ 284$ million, a decrease of $8.5 \%$.

DARPA plans to develop advanced multicast-based services to include refinements of collaboration systems and autonomous network processes. Emphasis will be placed on developing transport protocols, reference 
implementations, and demonstrations of applications such as real-time image understanding, target recognition, and autonomous navigation for reconnaissance and surveillance. DARPA, in collaboration with other agencies including NSA, will continue to develop an ATM network, with demonstrations of multimedia workstations and parallel computing technologies for medical imaging and scientific visualization. They will continue the development of optical switching and WDM devices to support up to $10 \mathrm{~Gb} / \mathrm{s}$ data rates. DARPA will finalize network control and management strategy of the WDM network and demonstrate WDM in a field trial at ATDnet. DARPA will deploy a reference implementation of a common base set of network infrastructure protocols and services necessary for secure and reliable network operation. Programs include wireless communications and wireless network access.

As a result of ongoing privatization, NSF will reduce support of commodity-level network services to educational institutions, and increase support of very high bandwidth services which focus on experimental applications of high bandwidth networks and international networking. Among the applications areas targeted for emphasis at NSF are distributed high performance computing, information based learning technologies, remote visualization and imaging, and tele-collaboration. NSF will support software systems research to enable the seamless convergence of computing and communications leading to a transparent information processing system. NSF will also support new applications of very high bandwidth communications demonstrating the integration of research and education. There will be increased emphasis on wireless communications and wireless network access, and on very broadband networks including optical networks.

The NASA projects formerly classified as IITA projects will all be completed in FY 1997, and no new projects in IITA will be started. NASA intends to upgrade the interconnect between the ESS and CAS testbeds to $622 \mathrm{Mb} / \mathrm{s}$. They will demonstrate network technologies needed for network management, cost accounting, security, and support of high performance applications in the NII.

The Digital Library projects, a joint research initiative with NSF, DARPA and NASA, will continue; related new work in very large databases and knowledge repositories will be supported.

DOE will prototype a system allowing multiple commercial building monitoring and operations from a single remotely sited control room. ESNet bandwidth services will be upgraded as budget allows, to support high speed Program Integration and Advanced Computational Testing and Simulation applications.

NSA will continue to develop LIGHTNING, a project to provide support for a WDM testbed. It will also develop an optically addressed spatial light modulator and complete development of the superconducting crossbar switch and associated electronics. It plans to transition out-of-band network control for ATM to multi-wavelength overlay terminal devices in anticipation of FY 1998-1999 testing of multi-wavelength switching devices on the ATDnet testbed multi-wavelength overlay. NSA will begin implementation and multi-network testing of interoperable network management over ATDnet and attached networks.

$\mathrm{NIH}$ will continue funding projects promoting the application of HPCC technologies to health care, the evaluation of telemedicine, and the testing of methods for protecting the privacy of electronic health data. There will also be a special emphasis on R\&D for computer-based patient records and public health applications of the NII. NIH will deploy new capabilities for automatic source selection and for retrieving and sorting information from multiple databases both within the Internet Grateful Med and Unified Medical Language System (UMLS). The scheduled increase in the number of funded genome centers will increase sequence data output to greater than 500,000 sequences per year. One of the goals of the program is to bring 3,000 health care institutions on to the Internet.

NIST will collaborate with industry and standards bodies to support the specification, development, validation, and testing of standards and interoperation of conforming products. NIST will initiate R\&D related to passive optical, non-ATM networks and R\&D related to coding and compression, and related testing technologies for wireless networks.

The VA intends to develop and test an interactive voice response system to enable access to information from existing VA computer systems 24 hours a day, 7 days a week. They will test and select clinical and administrative projects for migration to multiple facilities and develop a prototype epidemiological server for use by clinicians and clinical researchers. VA intends to enhance and test its Computerized Patient Record System. 


\subsubsection{LSN 1997 Expected Milestones}

- Demonstrate transport protocols for multi gigabit networks

- Demonstrate wide-area $40 \mathrm{~Gb} / \mathrm{s}$ and lab-prototype $100+\mathrm{Gb} / \mathrm{s}$ electro-optical transmission and switching systems

- Demonstrate integrating testbed architecture incorporating advanced distributed simulation, advanced distributed collaboration, advanced communications and control, and advanced human computer interfaces

- Demonstrate initial capabilities of intelligent information services architecture with multiple mechanisms for describing resource capabilities and with a uniform interface to hybrid search methods for resource retrieval

- Demonstrate a virtual reality advancement to allow a CAVE-to-CAVE interaction of at least twenty minutes

- Demonstrate WDM field trial at ATDnet with the final phase field trial at San Francisco Bay area

- Demonstrate integration of digital library technologies with remote sensing demonstrations

- Demonstrate completed Remote Sensing Database Applications over the Internet

- Demonstrate electronic switching of individual data flows at $2.4 \mathrm{~Gb} / \mathrm{s}$ data streams

- Demonstrate a $\mathrm{Gb} / \mathrm{s}$ Internet capability by connecting $\mathrm{Gb} / \mathrm{s}$ ATM networks with a $\mathrm{Gb} / \mathrm{s}$ IP router, with individual data flow of $2.4 \mathrm{~Gb} / \mathrm{s}$ over same

- Demonstrate as a system a soliton source and transmission, driving a couple of all-optical soliton-based logic gates, including the optical synchronization of the soliton transmission with the logic gate soliton signal

- Demonstrate bandwidth-adaptive multimedia node for mobile computing

- Demonstrate transparent application relocation within a mobile environment 


\begin{tabular}{|c|c|c|c|c|}
\hline $\begin{array}{r}\text { FY } 97 \\
\text { Total Pres } \\
\text { (BA } \quad \text { \$M) } \\
\end{array}$ & $\begin{array}{c}\text { FY } 96 \\
\text { Total Est } \\
(\mathrm{BA} \$ \mathrm{M}) \\
\end{array}$ & $\begin{array}{l}\text { Agency / Program Activity } \\
\text { Large Scale Networking }\end{array}$ & $\begin{array}{c}\text { FY } 97 \\
\text { Pres. } \\
\text { (BA \$M) }\end{array}$ & $\begin{array}{c}\text { FY } 96 \\
\text { Est } \\
\text { (BA \$M) }\end{array}$ \\
\hline \multirow[t]{5}{*}{115.93} & 96.04 & DARPA & & \\
\hline & & Networking Systems & 28.76 & 28.72 \\
\hline & & Defense Technology Integration and Infrastructure & $\mathbf{3 7 . 4 7}$ & 24.19 \\
\hline & & Global Mobile Infosystems & $\mathbf{1 7 . 5 8}$ & 16.30 \\
\hline & & Global Grid Communications & 32.12 & 26.83 \\
\hline \multirow[t]{16}{*}{72.26} & 104.47 & NSF & & \\
\hline & & NSFNET & 41.64 & 44.04 \\
\hline & & Networking, Communications and the Convergence of Computing \&Comm. & 19.92 & \\
\hline & & Applications & 8.20 & \\
\hline & & Education and Training & 2.50 & 2.50 \\
\hline & & Research Centers & & 0.68 \\
\hline & & Research Infrastructure & & 2.48 \\
\hline & & Ubiquitous Computing and Communication & & 14.44 \\
\hline & & Human-Machine Interaction \& Information Access & & 5.29 \\
\hline & & Biological Sciences (non-NC/GC) & & 12.42 \\
\hline & & Engineering (non-NC/GC) & & 1.38 \\
\hline & & Geosciences (non-NC/GC) & & 1.47 \\
\hline & & Computational Mathematics (non-NC/GC) & & 2.67 \\
\hline & & Physical Sciences (non-NC/GC) & & 6.41 \\
\hline & & Social, Behavioral \& Economic Sciences (non-NC/GC) & & 2.14 \\
\hline & & National Challenges & & 8.55 \\
\hline \multirow[t]{2}{*}{14.79} & 12.64 & DOE & & \\
\hline & & ESnet & 14.79 & 12.64 \\
\hline
\end{tabular}

Table 3: Large Scale Networking Program Activity Summary for FY 1997 Presidential Budget
Page 22 


\begin{tabular}{|c|c|c|c|c|}
\hline $\begin{array}{r}\text { FY } 97 \\
\text { Total Pres } \\
\text { (BA } \quad \$ M) \\
\end{array}$ & $\begin{array}{c}\text { FY 96 } \\
\text { Total Est } \\
(\mathrm{BA} \quad \$ \mathrm{M})\end{array}$ & $\begin{array}{l}\text { Agency / Program Activity } \\
\text { Large Scale Networking }\end{array}$ & $\begin{array}{c}\text { FY } 97 \\
\text { Pres. } \\
(\mathrm{BA} \$ \mathrm{M})\end{array}$ & $\begin{array}{c}\text { FY } 96 \\
\text { Est } \\
\text { (BA \$M) }\end{array}$ \\
\hline \multirow[t]{3}{*}{15.30} & 27.45 & NASA & & \\
\hline & & Grand Challenge Support & & 6.60 \\
\hline & & NREN & 15.30 & 20.85 \\
\hline \multirow[t]{12}{*}{22.90} & 21.51 & $\mathrm{NIH}$ & & \\
\hline & & NLM Medical Connections Program & 1.22 & 0.82 \\
\hline & & NLM Biotechnology Informatics & 4.92 & 4.54 \\
\hline & & NLM IAIMS grants & 2.00 & 2.00 \\
\hline & & NLM Intelligent Agent DB searching & 6.42 & 6.27 \\
\hline & & NLM HPCC Health Care Applications & 3.33 & 2.87 \\
\hline & & NCRR Biomolecular Computing & 0.20 & 0.20 \\
\hline & & NCRR Modeling/Simulation & 0.10 & 0.10 \\
\hline & & DCRT High Performance Biomedical Computing Program & 2.30 & 2.30 \\
\hline & & NCI Frederick Biomedical Supercomputing Center & 1.39 & 1.39 \\
\hline & & NCI High Speed Networking and Distributed Conferencing & 0.72 & 0.72 \\
\hline & & NCI High Perf. Comms for PDQ, CancerNet, and Electronic Publishing & 0.30 & 0.30 \\
\hline \multirow[t]{2}{*}{3.50} & 3.00 & NSA & & \\
\hline & & Very High Speed Networking & 3.50 & 3.00 \\
\hline \multirow[t]{4}{*}{2.96} & 2.20 & NIST & & \\
\hline & & Development and Dissemination of Scientific Software for HPCS & 0.60 & 0.50 \\
\hline & & Infrastructure for Information Technology & 1.86 & 1.20 \\
\hline & & Systems Integration for Manufacturing Applications & 0.50 & 0.50 \\
\hline \multirow[t]{3}{*}{9.45} & 14.13 & VA & & \\
\hline & & Computerized Patient Record and Telemedicine & 1.20 & 0.80 \\
\hline & & Clinical Workstations and Medical Imaging & 2.00 & 4.00 \\
\hline
\end{tabular}

Table 3: Large Scale Networking

Program Activity Summary for FY 1997 Presidential Budget 


\begin{tabular}{|c|c|c|c|c|}
\hline $\begin{aligned} \text { FY } 97 \\
\text { Total Pres } \\
\text { (BA } \quad \$ M) \\
\end{aligned}$ & $\begin{array}{c}\text { FY } 96 \\
\text { Total Est } \\
(\mathrm{BA} \quad \$ \mathrm{M}) \\
\end{array}$ & $\begin{array}{l}\text { Agency / Program Activity } \\
\text { Large Scale Networking }\end{array}$ & $\begin{array}{c}\text { FY } 97 \\
\text { Pres. } \\
\text { (BA \$M) }\end{array}$ & $\begin{array}{c}\text { FY } 96 \\
\text { Est } \\
\text { (BA \$M) }\end{array}$ \\
\hline & & Improve Telecommunications Infrastructure and Internet Connectivity & 0.50 & 0.18 \\
\hline & & VA Hybrid Open Systems Technology (VA HOST) & 4.75 & 8.50 \\
\hline & & VA/DoD Sharing & 1.00 & 0.65 \\
\hline \multirow[t]{2}{*}{2.70} & 2.70 & NOAA & & \\
\hline & & Networking Connectivity & 2.70 & 2.70 \\
\hline 259.79 & 284.14 & LSN FY 1997 Total & 259.79 & 284.14 \\
\hline
\end{tabular}

Table 3: Large Scale Networking 


\subsection{High Confidence Systems (HCS)}

\subsubsection{HCS Definition}

\section{As defined in the FY 1997 Blue Book:}

"HCS research will develop technologies that provide users with high levels of security, protection, reliability, and restorability of information services. Such systems are resistant to system failure and malicious penetration or damage and readily respond to interference by adaptation or recovery. These systems include both physical components, wired and wireless technologies, the data they contain and transmit, and the software that manipulates these data. HCS R\&D focuses on (I) system reliability (such as management of networks under load, failure, or intrusion; emergency response; firewalls; secure enclaves; and formal methods), (2) security and privacy (including personal identification, access control, authentication, encryption and other privacy assurance techniques, public key infrastructures, and trusted agents for secure distributed computing), and (3) testing and evaluation. Key applications include national security, law enforcement, life-and safety-critical requirements, personal privacy, and protection of critical elements of the National Information Infrastructure."

High confidence systems imply a level of reliability, privacy, and security necessary for a wide variety of applications, including:

- National defense secure systems

- Electronic commerce

- Remote operation of scientific instruments such as synchrotron light sources, telescopes, surgical instruments, undersea and outer space vehicles, and data collection systems

- Health care patient record exchange systems and telemedicine

Fields of high confidence systems research include fault tolerance, real time operation, security, and functional correctness. HCS research requires integrating these fields and creating standard metrics for properties such as safety, security, performance, and reliability. Developers need theories and tools to predict the confidence of different architectures to be able to use high confidence methods to compose high confidence components. The limits of composability must be explored to determine if there are collections of high confidence properties (e.g., security and performance) that cannot be derived from compositional principles.

A challenge is to develop an integrated security fabric in which trusted components can interact closely and rapidly, while mutually suspicious agents can cooperate through security gateways. Central to this challenge for security is scalability of such an integrated security fabric. Scalability is also critical for other properties of high confidence such as performance. Methods and tools such as automated and semi-automated verifiers have not been demonstrated for complex distributed systems, and as systems get ever more complex the issue of scalability becomes critical.

Mechanical verification tools already used for verifying important kernels must be extended to make them easier to use and interoperable for distributed operating systems. Research support for the development of educational programs for safety-critical systems is of vital importance. Areas once considered separate (safety, security, functional correctness, performance in real time, and fault tolerance) are parts of this emerging area. To improve the educational environment for high confidence systems, industry, government, and academia must organize curricula, case studies, electronic textbooks on the Internet, and shared software.

The HCS research includes support of the development of a suite of prototypical high confidence system specifications so that students in different universities can work on similar sets of reasonably realistic problems. This requires research into formulating the problems representative of problems faced in industry and government that are simple enough that students can develop at least partial solutions in a year.

A high confidence system has clearly defined usage and environmental parameter ranges. The benefits from using the system must exceed the costs of non-use, failures, and misuse. The user community must have a high level of confidence that a system will not perform incorrectly due to system errors, faults in the environment, or attempts to compromise the system. New technologies developed to ease the creation of critical high confidence systems can also be used to increase confidence and trust in all computing systems. 


\subsubsection{HCS Status}

Funds to support the HPCC portion of activities in High Confidence Systems for FY 1997 total \$32 million, compared to the FY 1996 Estimated budget of $\$ 30$ million, an increase of $6.7 \%$.

DARPA will demonstrate a prototype scalable operating system that incorporates high assurance capabilities for the Information Survivability program. They will also deploy a reference implementation of a common base set of network infrastructure protocols and services necessary for secure and reliable network operation.

NSA will release an expanded Synergy prototype including enhancements and improvements derived from collaboration with vendors and universities, to influence the design of commercial operating system development.

DOE will design and demonstrate adaptable high speed distributed computing communication libraries with multiple security and authentication, as well as performance, mechanisms.

NIST will begin an initiative in improved methods for software engineering, making use of powerful, mathematically-based techniques for analysis and synthesis of software systems. The goal is to establish laboratory facilities to conduct R\&D and assess candidate technologies that support automated testing and integrated software engineering. NIST will also initiate R\&D in coding and compression, as well as related testing technologies for wireless networks.

VA will expand testing of the Nationwide Authentication, Authorization, and Encryption Services to allow secure transmission of medical data across unsecured telecommunication links such as public networks, phone systems, and the Internet.

\subsubsection{HCS 1997 Expected Milestones}

- Demonstrate a prototype scalable operating system that incorporates high assurance capabilities supporting realtime, distributed, and limited fault-tolerant scalable computing applications

- Demonstrate and make available higher assurance components for distributed operating systems

- Develop architectures for high speed key management processors or servers

- Demonstrate an extended set of interface protocols and integration capabilities for the design, planning, and production of mechanical production

- Publish an extended series of interface specification documents for integrating software for manufacturing applications 


\begin{tabular}{|c|c|c|c|c|}
\hline $\begin{array}{c}\text { FY } 97 \\
\text { Total Pres } \\
\text { (BA } \$ \text { M })\end{array}$ & $\begin{array}{c}\text { FY } 96 \\
\text { Total Est } \\
(\mathrm{BA} \quad \$ \mathrm{M})\end{array}$ & $\begin{array}{l}\text { Agency / Program Activity } \\
\text { High Confidence Systems }\end{array}$ & $\begin{array}{c}\text { FY } 97 \\
\text { Pres. } \\
\text { (BA \$M) }\end{array}$ & $\begin{array}{c}\text { FY } 96 \\
\text { Est } \\
\text { (BA \$M) }\end{array}$ \\
\hline \multirow[t]{3}{*}{10.00} & 10.00 & DARPA & & \\
\hline & & Scalable Systems and Software & 5.00 & 5.00 \\
\hline & & Networking Systems & 5.00 & 5.00 \\
\hline \multirow[t]{2}{*}{1.21} & & NSF & & \\
\hline & & Computing Systems & 1.21 & \\
\hline \multirow[t]{2}{*}{1.00} & & NASA & & \\
\hline & & Grand Challenge Support & 1.00 & \\
\hline \multirow[t]{5}{*}{4.94} & 4.38 & $\mathrm{NIH}$ & & \\
\hline & & NLM Biotechnology Informatics & 0.75 & 0.68 \\
\hline & & NLM IAIMS grants & 0.50 & 0.50 \\
\hline & & NLM HPCC Health Care Applications & 3.55 & 3.06 \\
\hline & & NCI Frederick Biomedical Supercomputing Center & 0.14 & 0.14 \\
\hline \multirow[t]{3}{*}{7.30} & 7.40 & NSA & & \\
\hline & & Secure Operating System Development & 4.50 & 4.70 \\
\hline & & High Speed Data Protection Electronics & 2.80 & 2.70 \\
\hline \multirow[t]{3}{*}{5.20} & 5.36 & NIST & & \\
\hline & & Infrastructure for Information Technology & 4.20 & 4.20 \\
\hline & & Systems Integration for Manufacturing Applications & 1.00 & 1.16 \\
\hline \multirow[t]{6}{*}{2.30} & 2.90 & $\mathrm{VA}$ & & \\
\hline & & Computerized Patient Record and Telemedicine & 0.20 & 0.20 \\
\hline & & Clinical Workstations and Medical Imaging & 0.60 & 1.20 \\
\hline & & Improve Telecommunications Infrastructure and Internet Connectivity & 0.25 & \\
\hline & & VA Hybrid Open Systems Technology (VA HOST) & 1.00 & 1.50 \\
\hline & & VA/DoD Sharing & 0.25 & \\
\hline
\end{tabular}

Table 4: High Confidence Systems

Program Activity Summary for FY 1997 Presidential Budget 


\begin{tabular}{|c|c|c|c|c|}
\hline $\begin{array}{c}\text { FY } 97 \\
\text { Total Pres } \\
\text { (BA } \$ \mathrm{M}) \\
\end{array}$ & $\begin{array}{cc}\text { FY } & 96 \\
\text { Total } & \text { Est } \\
(\mathrm{BA} & \mathbf{\$ M})\end{array}$ & $\begin{array}{l}\text { Agency / Program Activity } \\
\text { High Confidence Systems }\end{array}$ & $\begin{array}{c}\text { FY } 97 \\
\text { Pres. } \\
\text { (BA \$M) }\end{array}$ & $\begin{array}{c}\text { FY } 96 \\
\text { Est } \\
\text { (BA \$M) }\end{array}$ \\
\hline 31.95 & 30.04 & HCS FY 1997 Total & 31.95 & 30.04 \\
\hline
\end{tabular}




\subsection{Human Centered Systems (HuCS)}

\subsubsection{HuCS Definition}

\section{As defined in the FY 1997 Blue Book:}

"HuCS R\&D makes computing systems and communications networks more easily accessible to and useable by a wide range of user communities. These communities include scientists and engineers, educators and students, the workforce, and the general public. Technologies enabling such systems include: (1) "knowledge repositories" and "information agents" for managing, analyzing, and presenting massive amounts of multimedia and multi-source information; (2) "collaboratories" that provide access to knowledge repositories and that facilitate knowledge sharing, group authorship, and control of remote instruments; (3) systems that enable multi-modal human system interactions including speech, touch, and gesture recognition and synthesis; and (4) virtual reality environments and their application to fields including scientific research, health care, manufacturing, and training."

"Knowledge repositories" are huge electronic databases that are being created for access by users ranging in size from small groups to the general public. The content is multimedia (text, voice, images, and video), and the data reside on distributed heterogeneous computing systems that use different database management software. There is a critical need for tools to manage these databases, as well as for "information agents" to analyze the data and present the results of this analysis most effectively. Analysis technologies include document translation and understanding. Technologies required for presenting these data and analyses include visualization, audio, and touch, and need to be selected by a user based on personal preference or need. Technologies that allow the user to interact with the information include virtual environments and telerobotics.

Collaboratories, which permit geographically distant people to work together and to use remote resources as if there were no geographical separation, build upon knowledge repositories and information agents. Collaboratories also require new technologies for creating multimedia information (for example, middleware for group authorship) and for controlling remote instruments that will enable researchers to access scarce and expensive research resources from their desktops.

Although today's graphical user interfaces are designed for a well-trained and unimpaired individual seated in front of a desktop monitor and keyboard, tomorrow's diverse user population requires user-friendly interfaces that are not only easy for the novice or infrequent user to use-regardless of physical ability, education, and culture-but remain powerful and productive for the expert. Enhanced Braille interfaces are needed by people who cannot see, and eyetracking technologies can be employed by people who cannot type. To this end, HuCS technologies will enable speech recognition and synthesis, as well as recognition by facial expression, touch, and gesture. Human-centered application development environments will allow users to tailor services and applications to meet individual needs. Other areas of HuCS research include interfaces to support computing systems embedded in household appliances and in wireless hand-held and wrist-mounted devices.

Virtual environments are a requirement for innovative knowledge presentation and collaboratories. They are interactive, computer-generated multi-dimensional models of real or artificial worlds designed so that the user's view of the environment changes in real time in response to user control. This control of viewpoint induces kinesthetic depth perception in which a user can perceive and interactively change a virtual world through direct manipulation of virtual objects in the environment. Intelligent real-time response and multi-dimensional sensory information can facilitate explorations involving complex tasks in science, medicine, and manufacturing. High-quality sensory information - that derived from sight, sound, and touch-is generated by computing systems and delivered to users by special interface devices. These devices provide the sensation that a user is interacting with an environment just as that person would interact with the real world, and in addition employ "intelligence amplification" to expand decision-making capabilities.

Virtual laboratories are virtual environments that facilitate scientific research by enabling interactive exploration in a three-dimensional environment of large, complex sets of multi-dimensional data. These data may be produced by modeling or simulation (for example, computational fluid dynamics used to model the flow of air or water), or may be empirical data (for example, from on-line experiments feeding observations to the researcher in real time), or both. This interactive exploration capability will enhance significantly the ability of the researcher to observe and understand scientific phenomena. 
Virtual environments promise to foster faster and more effective learning. By tailoring virtual worlds to specific educational or training tasks (for example flight simulators), people can be trained to use complex and costly equipment without acquiring that equipment or putting equipment and trainee at risk. Virtual laboratory infrastructures can be easily adapted to educational purposes.

HuCS R\&D in the area of virtual environments will include immersion in simulated environments, advanced modeling and simulation technologies, and group collaborations in virtual spaces. Expert systems that aid in the design of virtual environments are also required, so that complex environments appropriate to a specialized task can be automatically constructed. High quality visual displays, and possibly high-quality force and touch displays are required. Augmented-reality-based systems will require very accurate tracking and methods to closely align the virtual environment with the real world. Highly portable, wearable, virtual environment systems must he developed to support product maintenance in the field.

Used in knowledge presentations, collaboratories, and virtual environments, visualization systems and tools for multimodal presentations are key HuCS technologies. Advances are needed in feature classification, comparative visualization for trend analysis, merging of multisource data for visualization, combining data from multiple disciplines for visualization, integrated visualization of both source data and analysis products, and visualization for the blind.

Telemedicine consultation and treatment allows the patient and health care practitioners to be in different locations while the patient is examined, diagnosed, and one day treated remotely. In such activities, images and data from diverse medical instruments (for example, Magnetic Resonance Imaging, X-ray, and sonograms) will be integrated and displayed in a three-dimensional environment. In the longer term, remotely operated surgical robots may be used to perform operations, including unusual, difficult, or time-critical operations such as on a battlefield. Such technologies require substantial improvements over current HuCS technologies, including data management, accurate registration of remote instruments, and very accurate visual, touch, and force displays and tracking systems.

Other application areas include battlefield management and planning, emergency management, multi-government interactions, and industrial competition and collaboration. In each of these areas, human centered systems can provide an information-rich overview of highly complex situations involving many participants. HuCS technologies can be used to simulate these applications for training and evaluation purposes and to use real-time input to monitor actual situations for situation awareness and decision making. These applications also require special authoring tools for creating and maintaining simulations and the ability to partition a simulation into hierarchical levels of detail. In product manufacturing, for example, these technologies can support the total life cycle of a manufactured product, resulting in improved design, development, testing, manufacture, training and use, and maintenance and repair.

\subsubsection{HuCS Status}

Funds to support activities in Human Centered Systems for FY 1997 total \$249 million, compared to the FY 1996 Estimated budget of $\$ 188$ million, an increase of $32 \%$.

Major activities are reflected in DARPA programs in Intelligent Systems, where the intent is to develop modular human language technologies to support easy, low-cost, rapid technology transfer and application development for document understanding, machine translation, and speech understanding. In the Intelligent Integration of Information area, DARPA will support the development of tools and techniques to enable the rapid construction of information fusion, aggregation, and summarization software. DARPA will extend and evaluate large-scale statistical modeling, machine learning, and knowledge representation methods for spoken and written language understanding and develop hub formalization that will infuse existing programming languages with new advances in formal methods. They will continue the experimental evaluation of design technology for high performance computational prototyping of systems.

NSF, NASA, and DARPA will continue the Digital Library projects. This research seeks to develop real-time image understanding algorithms for use in image registration, target recognition, and autonomous navigation for ground level and overhead reconnaissance and surveillance and to implement initial tools and toolkits for development and evaluation of highly interactive, agent and dialogue-based human computer interactions.

The NASA IITA program, which includes some HuCS activities, will be completed in FY 1997. NASA, working collaboratively with other Federal agencies whose primary focus is HuCS, will continue to invest in the area of 
Human Centered Systems using expertise from its Information Technology Center of Excellence. These investments will be through some of NASA's more traditional efforts such as computational aerosciences.

NSF will increase support for research in information-based learning technologies with the potential to transform education at all levels in the 21st century and form a new enabler for the integration of research and education.

NSF will initiate the multi-agency program STIMULATE (Speech, Text, Image, and MULtimedia Advanced Technology Effort) in order to understand multimodal human communication and apply it to computer technology.

NIH will continue its Telemedicine and Visible Human programs and develop and test a graphical user interface for an existing medical imaging system. NLM will begin projects for the full object identification of the Visible Human data sets. NCRR will define the requirements to establish and evaluate two or three collaboratory testbeds (possibly with NSF and DARPA as partners).

DOE will provide a prototype integrated, distributed multimedia scientific visualization environment for HPCC researchers by integrating existing collaborative tools into a virtual Laboratory Framework.

NIST will support projects on models, architectures, conformance testing and collaboratory technology supporting manufacturing integration, information metrology, and improvement in the accessibility of standard reference data and algorithms for scientists, educators, the workforce and the public.

ED's National Institute on Disability and Rehabilitation will continue funding of 15 continuing and one new Rehabilitation Engineering Research Centers (RERCs). These centers support programs designed to conduct research, demonstration, and training activities. RERCs focus on issues dealing with rehabilitation technology, including rehabilitation engineering and assistive technology devices and services.

AHCPR supports research into the barriers to a successful installation of comprehensive health information systems, emphasizing the speed, cost, and human factors that affect success-to accelerate the transfer of computer-based information technology. They will evaluate the medical effectiveness and economic impact (cost benefits) of automated clinical decision support systems in diverse health care settings.

\subsubsection{HuCS 1997 Expected Milestones}

- Demonstrate integrating testbed architecture incorporating advanced distributed simulation, advanced distributed collaboration, advanced communications and control, and advanced human computer interfaces

- Demonstrate enhanced feature, real-time distributed operating systems for embedable high performance computing systems

- Demonstrate initial capabilities of intelligent information services architecture with multiple mechanisms for describing resource capabilities and with a uniform interface to hybrid search methods for resource retrieval

- Demonstrate initial multisite collaborative design research environment for integrated circuit process simulation and remote experimentation over the Internet

- Demonstrate feasibility of utilizing advanced software environment that supports composition tools for composing software, integration, and software development and testing using animation techniques in military environment

- Demonstrate desktop virtual reality for Earth and Space Science Investigator applications

- Implement the prototype for the complete second National Health and Nutrition Examination Survey (NHANES II) image data base

- Demonstrate and evaluate new applications for prototype systems of virtual environments for scientific instruments

- Demonstrate an extended set of interface protocols and integration capabilities for the design, planning, and production of mechanical systems

- Develop and demonstrate modular human language technologies to support easy, low cost, rapid technology transfer and application development for document understanding, machine translation, and speech understanding

- Develop and demonstrate high performance, collaborative, knowledge-acquisition tools for problem solving tasks and decision aids

- Initiate new, inter-disciplinary research projects in STIMULATE across several agencies 


\begin{tabular}{|c|c|c|c|c|}
\hline $\begin{array}{rr}\text { FY } 97 \\
\text { Total } \\
\text { (BA } & \$ M \\
\end{array}$ & $\begin{array}{c}\text { FY } 96 \\
\text { Total Est } \\
(\mathrm{BA} \quad \$ \mathrm{M}) \\
\end{array}$ & $\begin{array}{l}\text { Agency / Program Activity } \\
\text { Human Centered Systems }\end{array}$ & $\begin{array}{c}\text { FY } 97 \\
\text { Pres. } \\
\text { (BA \$M) }\end{array}$ & $\begin{array}{c}\text { FY } 96 \\
\text { Est } \\
\text { (BA \$M) }\end{array}$ \\
\hline \multirow[t]{6}{*}{117.03} & 112.17 & DARPA & & \\
\hline & & Microsystems & 18.21 & 17.05 \\
\hline & & System Environments & 2.18 & 3.68 \\
\hline & & Defense Technology Integration and Infrastructure & 5.24 & 9.01 \\
\hline & & Information Sciences & 19.26 & 20.50 \\
\hline & & Intelligent Systems and Software & 72.14 & 61.93 \\
\hline \multirow[t]{3}{*}{57.76} & & NSF & & \\
\hline & & Human Centered Systems & 48.10 & \\
\hline & & Applications & 9.66 & \\
\hline \multirow[t]{3}{*}{12.98} & 8.56 & DOE & & \\
\hline & & National Collaboratory Research & 7.98 & 8.38 \\
\hline & & DOE2000 NC & 5.00 & 0.18 \\
\hline \multirow[t]{2}{*}{3.40} & & NASA & & \\
\hline & & Systems Software & 3.40 & \\
\hline \multirow[t]{10}{*}{29.59} & 24.34 & NIH & & \\
\hline & & NLM Biotechnology Informatics & 0.50 & 0.45 \\
\hline & & NLM Electronic Imaging & 1.44 & 1.44 \\
\hline & & NLM IAIMS grants & 0.90 & 0.90 \\
\hline & & NLM Intelligent Agent DB searching & 2.15 & 2.10 \\
\hline & & NLM HPCC Health Care Applications & 13.98 & 12.03 \\
\hline & & NCRR Biomolecular Computing & 0.80 & 0.60 \\
\hline & & NCRR Modeling/Simulation & 0.70 & 1.20 \\
\hline & & NCRR Virtual Reality/Environments & $\mathbf{7 . 7 0}$ & 4.20 \\
\hline & & DCRT High Performance Biomedical Computing Program & $\mathbf{0 . 5 0}$ & 0.50 \\
\hline
\end{tabular}

Table 5: Human Centered Systems

Program Activity Summary for FY 1997 Presidential Budget 


\begin{tabular}{|c|c|c|c|c|}
\hline $\begin{array}{l}\text { FY } 97 \\
\text { Total Pres } \\
\text { (BA } \$ M \text { ) }\end{array}$ & $\begin{array}{c}\text { FY 96 } \\
\text { Total Est } \\
\text { (BA } \quad \text { \$M) } \\
\end{array}$ & $\begin{array}{l}\text { Agency / Program Activity } \\
\text { Human Centered Systems }\end{array}$ & $\begin{array}{c}\text { FY } 97 \\
\text { Pres. } \\
\text { (BA \$M) }\end{array}$ & $\begin{array}{c}\text { FY } 96 \\
\text { Est } \\
\text { (BA } \$ M) \\
\end{array}$ \\
\hline & & NCI Frederick Biomedical Supercomputing Center & 0.28 & 0.28 \\
\hline & & NCI High Speed Networking and Distributed Conferencing & 0.34 & 0.34 \\
\hline & & NCI High Perf. Comms for PDQ, CancerNet, and Electronic Publishing & 0.30 & 0.30 \\
\hline \multirow[t]{3}{*}{9.56} & 9.86 & NIST & & \\
\hline & & Infrastructure for Information Technology & 2.20 & 2.86 \\
\hline & & Systems Integration for Manufacturing Applications & 7.36 & 7.00 \\
\hline \multirow[t]{2}{*}{11.40} & 11.40 & $\mathrm{ED}$ & & \\
\hline & & National Institute on Disability and Rehabilitation Research & 11.40 & 11.40 \\
\hline \multirow[t]{4}{*}{1.80} & 1.90 & $\mathrm{VA}$ & & \\
\hline & & Computerized Patient Record and Telemedicine & 0.40 & 0.40 \\
\hline & & Clinical Workstations and Medical Imaging & 0.40 & \\
\hline & & VA Hybrid Open Systems Technology (VA HOST) & 1.00 & 1.50 \\
\hline \multirow[t]{2}{*}{0.50} & 0.50 & NOAA & & \\
\hline & & Information Dissemination Pilots & 0.50 & 0.50 \\
\hline \multirow[t]{2}{*}{0.60} & 0.60 & EPA & & \\
\hline & & Public Data Access & 0.60 & 0.60 \\
\hline \multirow[t]{2}{*}{4.20} & 3.20 & AHCPR & & \\
\hline & & Computer-Based Patient Records & 4.20 & 3.20 \\
\hline 248.82 & 172.53 & HuCS FY 1997 Total & 248.82 & 172.53 \\
\hline
\end{tabular}




\title{
4.6. Education, Training, and Human Resources (ETHR)
}

\subsubsection{ETHR Definition}

\begin{abstract}
As defined in the FY 1997 Blue Book:
"The focus of ETHR R\&D is on education and training technologies. The goals of this education and training are to produce (1) researchers and students in high performance computing, communications, information technologies, and their application, and (2) a citizenry with the skills to compete and prosper in the 21st century's information age. ETHR includes curriculum development, fellowships, and scholarships for computational, computer, and information sciences and engineering. It includes the application of interdisciplinary research to learning technologies, and $R \& D$ in information-based learning tools, lifelong learning, and distance learning for people in remote locations."
\end{abstract}

One major goal of the CIC's R\&D program is accelerating the use of high performance computing, communications, and information technologies in U.S. education. Citizens should be prepared with the knowledge, skills, and insights to lead research in science and technology and the ability to apply the resulting discoveries to industrial needs. This activity complements the mission of the NSTC's Committee on Education and Training (CET), which is formulating strategies to raise the science and mathematical skills of all Americans. All citizens should be able to become fully engaged in the technological society of the future. In collaboration with the CET, the CIC will sponsor research in advanced information and learning technologies to establish a foundation for a new educational methodology.

Educational applications build on all of the computing, information, and communications technologies described above. These applications demand ease of use, interfaces that span a wide range of user sophistication, access to distributed collections of information and expertise, ease of authoring new materials, ease of indexing existing collections, low cost network access, management capabilities that must scale, extensive use of simulation, visualization, and virtual reality technologies to support training. They also demand privacy, security, protection of the learning infrastructure, intellectual property protection for authored materials, and associated billing and payment systems.

Research is needed to accelerate the development of educational authoring and presentation tools that provide seamless, friendly, portable, mobile, easy-to-use and easy-to-maintain environments for learning, and strong links to Human-Centered Systems. Learning environments provide particular challenges in the user interface arena since interfaces are needed for learners at all levels of sophistication.

Techniques for improving the efficiency of digitizing holdings in our national archives and museums, of critical interest in Large-Scale Networking Technologies, would also have great benefit for educational technologies. Navigation assistants to help educational users browse through huge databases touch on both LSN and HuCS.

\subsubsection{ETHR Status}

Funds to support activities in Education, Training, and Human Resources for FY 1997 total \$45 million, compared to the FY 1996 Estimated budget of $\$ 90$ million, a decrease of $50 \%$.

In ED, the Education Resources Information Center (ERIC) will continue to maintain, and provide access to, the world's largest electronic bibliographic database of education-related publications and documents through a national system of specialist clearing houses. The ERIC system provides access to its network of bibliographic information to educators, policy makers, parents and the general public. It provides access to the database clearinghouses via mail, telephone, and electronic routes. It maintains a question and answer service via the Internet. Improvements are planned in two areas: providing increased access through the Internet node (gopher and WWW) to the National Library's repository of information about ED programs, projects, publications, and statistics by beginning the development of a digital library; and actively disseminating syntheses of research and development findings and other materials through electronic networking.

DARPA will facilitate transition of the Combat Care Associate software to emergency services.

NSF no longer reports the Directorate for Education and Human Resources budget as part of the HPCC Program, but the educational activity has not decreased in that agency. NSF will continue support of the Grand Challenges and National Challenges initiated in FY 1993, FY 1994, and FY 1995. It will also continue to support the paradigm shift 
involving increased use of high performance computing to enhance or replace the experimental phase of the scientific method.

NASA will demonstrate education products from K-14 Education Cooperative Agreement Notice and issue a second K-14 Education Cooperative Agreement.

NIH/NCRR will continue to provide National Research Service Awards (NRSA) for formalized training for biomedical scientists in the use of high performance computing. NIH/NCI will apply computational chemistry to understanding of drug interactions with enzymes and nucleic acids as a basis for discovery of new drugs and provide support for increasing numbers of users in extramural and intramural research in biomedical computing.

In the DOE Advances in Supercomputing program, the number of state sites will be reduced from five to the original three.

\subsubsection{ETHR 1997 Expected Milestones}

- Demonstrate protocol based care in all battlefield and peacetime settings

- Demonstrate performance gains of advanced software engineering collaborators

- Demonstrate improved life cycle systems management using SEP/DSSA (domain-specific software architecture)

- Initiate a new program in Learning Technologies that will combine human centered information systems research with research in education to provide the basis for the next generation of technologies for education 


\begin{tabular}{|c|c|c|c|c|}
\hline $\begin{array}{r}\text { FY } 97 \\
\text { Total Pres } \\
\text { (BA } \$ \mathrm{M}) \\
\end{array}$ & $\begin{array}{c}\text { FY } 96 \\
\text { Total Est } \\
\text { (BA } \$ \text { M ) }\end{array}$ & $\begin{array}{r}\text { Agency / Program Activity } \\
\text { Education, Training, and Human Resources }\end{array}$ & $\begin{array}{c}\text { FY } 97 \\
\text { Pres. } \\
\text { (BA \$M) }\end{array}$ & $\begin{array}{c}\text { FY } 96 \\
\text { Est } \\
\text { (BA \$M) }\end{array}$ \\
\hline \multirow[t]{2}{*}{7.51} & 6.24 & DARPA & & \\
\hline & & Health Information Infrastructure & 7.51 & 6.24 \\
\hline \multirow[t]{8}{*}{19.11} & 31.03 & NSF & & \\
\hline & & Applications & 11.86 & \\
\hline & & Education and Training & 7.25 & 14.12 \\
\hline & & Research Centers & & 4.40 \\
\hline & & Research Infrastructure & & 2.30 \\
\hline & & Human-Machine Interaction \& Information Access & & 3.60 \\
\hline & & Computational Mathematics (non-NC/GC) & & 3.52 \\
\hline & & Physical Sciences (non-NC/GC) & & 3.09 \\
\hline \multirow[t]{2}{*}{3.50} & 4.00 & DOE & & \\
\hline & & Advanced Computational Testing and Simulation Research & 3.50 & 4.00 \\
\hline \multirow[t]{5}{*}{3.20} & 23.60 & NASA & & \\
\hline & & Grand Challenge Support & & 0.62 \\
\hline & & BRHR & 3.20 & 3.98 \\
\hline & & Information Infrastructure Technology & & 8.80 \\
\hline & & Information Infrastructure Applications & & 10.20 \\
\hline \multirow[t]{7}{*}{5.38} & 7.11 & $\mathrm{NIH}$ & & \\
\hline & & NLM HPCC Training Grants & 3.04 & 3.04 \\
\hline & & NCRR HPCC Training & 1.80 & 3.50 \\
\hline & & NCI Frederick Biomedical Supercomputing Center & 0.49 & 0.49 \\
\hline & & NCI High Speed Networking and Distributed Conferencing & 0.05 & 0.08 \\
\hline & 0.15 & NSA & & \\
\hline & & Technology Based Training & & 0.15 \\
\hline
\end{tabular}

Table 6: Education, Training, and Human Resources

Program Activity Summary for FY 1997 Presidential Budget 


\begin{tabular}{|c|c|c|c|c|}
\hline $\begin{array}{l}\text { FY } 97 \\
\text { Total Pres } \\
(\mathrm{BA} \$ \mathrm{M}) \\
\end{array}$ & $\begin{array}{l}\text { FY } 96 \\
\text { Total Est } \\
\text { (BA } \$ M \text { ) }\end{array}$ & $\begin{array}{r}\text { Agency / Program Activity } \\
\text { Education, Training, and Human Resources }\end{array}$ & $\begin{array}{c}\text { FY } 97 \\
\text { Pres. } \\
(\text { BA } \$ \text { M) }\end{array}$ & $\begin{array}{c}\text { FY } 96 \\
\text { Est } \\
\text { (BA } \$ M)\end{array}$ \\
\hline \multirow[t]{9}{*}{6.61} & 17.53 & ED & & \\
\hline & & AskERIC Service & 1.00 & 0.34 \\
\hline & & OERI Institutional Communications Network (INET) & 1.90 & 0.93 \\
\hline & & Regional Education Laboratory Program & 1.50 & 1.50 \\
\hline & & Teacher Networking Project & & 13.21 \\
\hline & & National Parents Information Network & 0.23 & 0.20 \\
\hline & & ERIC Clearinghouses & 1.98 & 1.35 \\
\hline & 0.08 & EPA & & \\
\hline & & Education/Training & & 0.08 \\
\hline 45.31 & 89.74 & ETHR FY 1997 Total & 45.31 & 89.74 \\
\hline
\end{tabular}

ज

Table 6: Education, Training, and Human Resources

Program Activity Summary for FY 1997 Presidential Budget

Page 37 


\section{Appendix A. Agency Accomplishments and Plans}

Implementation of the HPCC Program takes place within each of the twelve HPCC agencies, where the final decisions are made on the funding, management, and evaluation of program activities. This section organizes HPCC activities by agency to reflect the operational structure of the funding. The level of information does not reflect individual projects. Since many grants are at the level of $\$ 100,000-\$ 300,000$, a full breakout of each project in a $\$ 1$ billion program is impractical to assemble and maintain for this plan. Agencies group together projects that are technically related to reaching similar goals and objectives, as described in the PCAs. Each grouping constitutes a "program activity," which range in size from $\$ 0.1 \mathrm{M}$ to over $\$ 100 \mathrm{M}$.

Although this structure tracks funds clearly, it has several drawbacks. Interagency collaborations are often difficult to recognize and may in fact appear as duplication of effort, since similar efforts may be reported within several agencies' activities. Also, although the HPCC Program broadly describes activities under the heading of five major program component areas, actual program activities often span multiple areas, thus making the actual funding difficult to categorize. Because program activities need not follow strict guidelines of funding within each focus area, program managers make better decisions, at finer levels of program implementation, to determine the most effective use of research funds to meet Program objectives.

Each agency section contains three parts:

- Brief overview of the agency's perspective on HPCC efforts,

- Table summarizing financial data and collaborative ties for its program activities, and

- One- or two-page form describing each program activity and its milestones.

The table at the beginning of each agency's section of the report summarizes the financial data by activity and indicates collaborative ties.

The individual program activity form contains some fields that require explanation. The "Budget Code" field is an internal label used by some agencies to track funding. The budget fields provide actual spending (Act.), estimated spending based on Congressional appropriations and rescissions (Est.), and Presidential requests (Pres.) for various fiscal years. The numbers below the HPCC component labels estimate the breakdown of the FY 1997 request into the five PCAs. These numbers are estimates, in that many activities span a variety of PCAs and categorization of efforts includes subjective judgment.

The "Agency Ties" section of the form permits two. labels. A "Partner" label indicates that the corresponding agency participates in this activity as a funding and research partner. Note that two different agencies may view partnering differently (e.g., developing a scalable system in DARPA would be HECC, but its use for National Challenges could be inside LSN in NIST or NOAA). A "User" label indicates that the corresponding agency needs the results of this program activity for another, related activity. The large field in the center of the form gives an overview description of each activity. The remaining fields provide highlights of major accomplishments and milestones planned for future years. These entries highlight significant objectives and results; they should not be viewed as complete descriptions of any activity. 


\section{Defense Advanced Research Projects Agency}

DARPA is the lead DoD agency for advanced technology research, and has the leadership responsibility for the HPCC program within DoD. This is based on DARPA's history of technical innovations in computer architecture, integrated circuits, networking, and system software. DARPA's strategy is to focus on developing the underlying technology base for high performance computing and communications while other agencies apply these technologies within the context of their mission-specific application focus.

The High End Computing and Computation (HECC) activity focuses on developing high performance technologies (both hardware and software) for computing. Computing elements of the program are aimed at producing scalable software and architectures to meet defense computing requirements.

The Large Scale Networking (LSN) activity focuses on distributed services over broadly based, large scale interconnections of networks. Efforts are aimed at information management, secure transaction support, and integrated testbeds including special defense applications. The network elements are aimed at interoperability, mobility, and high performance systems.

The Human Centered Systems (HuCS) activity includes language understanding, knowledge representation, intelligent systems and human computer interaction. Virtual environments addresses distributed design environments, languages, and experimental applications.

The Education, Training, and Human Resources (ETHR) activity addresses intelligent software systems for combat casualty care.

The High Confidence Systems (HCS) activity addresses technologies for increasing systems reliability and recoverability under conditions of load, failure and intrusion. 
Defense Advanced Research Projects Agency

$\stackrel{\infty}{\infty}$

\begin{tabular}{|c|c|c|c|c|c|c|c|c|c|c|c|}
\hline \multirow[b]{2}{*}{ Program Activity } & \multirow[b]{2}{*}{ Budget Account Code } & \multirow{2}{*}{$\begin{array}{l}\text { Partner/User } \\
\text { Agencies }\end{array}$} & \multicolumn{4}{|c|}{ Budget $(\mathrm{BA}, \$ \mathrm{M})$} & \multicolumn{5}{|c|}{ HPCC PCAs by 1997 Pres. Request } \\
\hline & & & $\begin{array}{l}\text { FY } 95 \\
\text { Actual }\end{array}$ & $\begin{array}{c}\text { FY } 96 \\
\text { Pres }\end{array}$ & $\begin{array}{c}\text { FY } 96 \\
\text { Est. }\end{array}$ & $\begin{array}{l}\text { FY } 97 \\
\text { Rast }\end{array}$ & HECC & LSN & HCS & HuCS & ETHR \\
\hline Scalable Systems and Software & ST-19 & All HPCC & 52.45 & 46.70 & 37.63 & 32.17 & 27.17 & & 5.00 & & \\
\hline Microsystems & ST. 19 & NSF, NSA & 33.64 & 31.87 & 33.30 & 32.50 & 14.29 & & & 18.21 & \\
\hline System Environments & ST-19 & $\begin{array}{l}\text { All HPCC } \\
\text { Anencies }\end{array}$ & 35.15 & 24.62 & 21.96 & 17.68 & 15.50 & & & 2.18 & \\
\hline Networking Systems & ST- 19 & $\begin{array}{l}\text { NSF, DOE, } \\
\text { NASA }\end{array}$ & 35.31 & 31.43 & 33.72 & 33.76 & & 28.76 & 5.00 & & \\
\hline Defense Technology Integration and Infrastructure & ST-19 & NSF & 22.35 & 23.25 & 41.40 & 56.71 & 14.00 & 37.47 & & 5.24 & \\
\hline $\begin{array}{l}\text { Defense Technology Integration and Infrastructure } \\
\text { centined) }\end{array}$ & ST -19 & NSF & & & & & & & & & \\
\hline Global Mobile Infosystems & ST-19 & & 0.00 & 0.00 & 16.30 & 17.58 & & 17.58 & & & \\
\hline Information Survivability & ST-24 & $\begin{array}{l}\text { NSF, DOE, } \\
\text { NASA }\end{array}$ & 9.88 & & & & & & & & \\
\hline Health Information Infrastructure & MPT-07 & $\mathrm{NIH}$ & 9.11 & 10.24 & 6.24 & 7.51 & & & & & 7.51 \\
\hline Information Sciences & $\mathrm{CCS}-02$ & NSF & 20.56 & 23.72 & 23.10 & 23.35 & 4.09 & & & 19.26 & \\
\hline Intelligent Systems and Software & ST-11 & & 59.21 & 77.34 & 61.93 & 72.14 & & & & 72.14 & \\
\hline Intelligent Systems and Software (continued) & ST-11 & & & & & & & & & & \\
\hline Global Grid Communications & EE-45 & NSA & 21.79 & 26.83 & 26.83 & 32.12 & & 32.12 & & & \\
\hline Integrated Command and Control Technology & IC-03 & & 6.00 & 0.00 & & & & & & & \\
\hline Defense Information Enterprise Technologies & ST-19 & $\begin{array}{l}\text { NSF, NASA, } \\
\text { MIST }\end{array}$ & 29.61 & 57.61 & & & & & & & \\
\hline & & Totals: & 335.06 & 353.61 & 302.41 & 325.52 & 75.05 & 115.93 & 10.00 & 117.03 & 7.51 \\
\hline
\end{tabular}




\section{Defense Advanced Research Projects Agency}

\begin{tabular}{|c|c|c|c|c|c|c|c|c|c|c|c|c|c|c|}
\hline \multirow[b]{2}{*}{ Program Activity } & \multicolumn{4}{|c|}{ Budget $(\mathrm{BA}, \$ \mathrm{M})$} & \multicolumn{5}{|c|}{ HPCC PCAs by 1997 Pres. Request } & \multicolumn{5}{|c|}{ HPCC PCAs by 1996 Estimated } \\
\hline & $\begin{array}{l}\text { FY 95 } \\
\text { Actual }\end{array}$ & $\begin{array}{c}\text { FY } 96 \\
\text { Pres. }\end{array}$ & $\begin{array}{c}\text { FY } 96 \\
\text { Est. }\end{array}$ & $\begin{array}{l}\text { FY } 97 \\
\text { Rast. }\end{array}$ & HECC & LSN & HCS & HuCS & ETHR & HECC & LSN & HCS & HuCS & ETHR \\
\hline Scalable Systems and Software & 52.45 & 46.70 & 37.63 & 32.17 & 27.17 & & 5.00 & & & 32.63 & & 5.00 & & \\
\hline Microsystems & 33.64 & 31.87 & 33.30 & 32.50 & 14.29 & & & 18.21 & & 16.25 & & & 17.05 & \\
\hline System Environments & 35.15 & 24.62 & 21.96 & 17.68 & 15.50 & & & 2.18 & & 18.28 & & & 3.68 & \\
\hline Networking Systems & 35.31 & 31.43 & 33.72 & 33.76 & & 28.76 & 5.00 & & & & 28.72 & 5.00 & & \\
\hline Defense Technology Integration and Infrastructure & 22.35 & 23.25 & 41.40 & 56.71 & 14.00 & 37.47 & & 5.24 & & 8.20 & 24.19 & & 9.01 & \\
\hline $\begin{array}{l}\text { Defense Technology Integration and Infrastructure } \\
\text { (cantinued) }\end{array}$ & & & & & & & & & & & & & & \\
\hline Global Mobile Infosystems & 0.00 & 0.00 & 16.30 & 17.58 & & 17.58 & & & & & 16.30 & & & \\
\hline Information Survivability & 9.88 & & & & & & & & & & & & & \\
\hline Health Information Infrastructure & 9.11 & 10.24 & 6.24 & 7.51 & & & & & 7.51 & & & & & 6.24 \\
\hline Information Sciences & 20.56 & 23.72 & 23.10 & 23.35 & 4.09 & & & 19.26 & & 2.60 & & & 20.50 & \\
\hline Intelligent Systems and Software & 59.21 & 77.34 & 61.93 & 72.14 & & & & 72.14 & & & & & 61.93 & \\
\hline Intelligent Systems and Software (continued) & & & & & & & & & & & & & & \\
\hline Global Grid Communications & 21.79 & 26.83 & 26.83 & 32.12 & & 32.12 & & & & & 26.83 & & & \\
\hline Integrated Command and Control Technology & 6.00 & 0.00 & & & & & & & & & & & & \\
\hline Defense Information Enterprise Technologies & 29.61 & 57.61 & & & & & & & & & & & & \\
\hline Totals: & 335.06 & 353.61 & 302.41 & 325.52 & 75.05 & 115.93 & 10.00 & 117.03 & 7.51 & 77.96 & 96.04 & 10.00 & 112.17 & 6.24 \\
\hline
\end{tabular}


The Scalable Systems and Software program supports the development of computing and advanced software technologies needed to enable the $O$ development, introduction, and effective use of secure scalable and distributed high performance computing technologies. The program accelerates the evolution of computing and communications technologies. The high performance computing technologies developed by this program drive national defense capabilities in areas that benefit from the ability to do complex modeling and secure distributed communication.

Prototype Scalable Systems is focused on developing, demonstrating, and transferring the scalable computing systems technology base. Projects in this area cover a broad range of computing systems technology issues from research into future generation computing systems technology to the validation of scalable concepts through supporting the incorporation of advanced technology into industrial prototypes.

Operating Systems and Services supports the development of advanced software technologies to enable the development, introduction, and effective use of secure, scalable and distributed high performance computing technologies. In the past, it was sufficient to develop a "fast" computer system in the absence of a software environment. This is no longer the case as the time between generations of advanced hardware has been reduced from many years to less than two years, making it essential to maintain a stable software development environment that can span several generations of computer hardware. This is an absolutely mandatory need for DoD which cannot afford to redevelop software for each new generation of computers.

Budget (\$ M)

\begin{tabular}{|c|c|}
\hline FY 95 Act & 52.45 \\
\hline FY 96 Pres. & 46.70 \\
\hline FY 96 Est. & 37.63 \\
\hline FY 97 Rqst. & 32.17 \\
\hline
\end{tabular}

Program

Component

Areas

FY 96 FY 97

\begin{tabular}{l|l|l|l|} 
HECC & 32.63 & 27.17 \\
\hline
\end{tabular}

Milestone FY 96 milestone for scalable OS moved from System Environments

Changes
FY 1995 Actual Milestones

Designed system architectures incorporating components such as programmable protocol engines to support scalability and high performance.

Demonstrated systems tools for on-line analysis of a real-time operating systems for scalable, distributed HPC systems.

Demonstrated operating system ability to confine processes to isolated domains.

Demonstrated first HPC single node operating at 1 GFLOP/s.

\section{FY 1996 Estimated Milestones \\ Demonstrate high-availability systems scalable in performance to 1 teraFLOP/s.}

Demonstrate extensible modular operating system framework supporting real-time, distributed, and limited fault-tolerant scalable computing applications.

Demonstrate user-extensible microkernel operating system technology, integrating compiler and run-time support services.

Demonstrate computing node architectures that dramatically increase internal memory and communications bandwidths.

Demonstrate $\mathrm{I} / \mathrm{O}$ enhancements to a scalable operating system that overcomes identified bottlenecks leading to significant improvements in throughput.

Evaluate first generation of fully scalable OS software and programming environments on small-scale versions of teraops computing systems.
FY 1997 Agency Request Milestones Demonstrate scalability from distributed

workstation clusters to teraFLOP supercomputers on the identical technology base.

Demonstrate distributed cluster technology scalable to teraFLOPS.

Demonstrate advanced object management systems integrated with operating systems and applications to achieve efficient use of memory while enhancing execution speed.

Demonstrate the prototype of a scalable operating system that incorporates high assurance capabilities for the Defensive Information Warfar program

Demonstrate extensible modular operating system framework supporting real-time,

distributed, and limited fault-tolerant scalable computing applications.

\begin{tabular}{|c|c|c|}
\hline LSN & & \\
\hline HCS & 5.00 & 5.00 \\
\hline HuCS & & \\
\hline ETHR & & \\
\hline & ency & ies \\
\hline DARPA & & \\
\hline NSF & & ner \\
\hline DOE & & ner \\
\hline NASA & & ner \\
\hline NIH & & ner \\
\hline NSA & & ner \\
\hline NIST & & ner \\
\hline NOAA & & ner \\
\hline EPA & & ner \\
\hline ED & & \\
\hline$\overline{A H C P R}$ & & \\
\hline VA & & \\
\hline
\end{tabular}




\begin{tabular}{|c|c|}
\hline & $\forall \Lambda$ \\
\hline & УdगHV \\
\hline & स् \\
\hline & $\forall d G$ \\
\hline & $\forall \forall O N$ \\
\hline & LSIN \\
\hline \multirow[t]{4}{*}{. } & VSN \\
\hline & HIN \\
\hline & $\forall S V N$ \\
\hline & GOO \\
\hline Jәuน" & ASN \\
\hline & $\forall d \& \forall a$ \\
\hline
\end{tabular}

Sə!ป א๖uวశี V

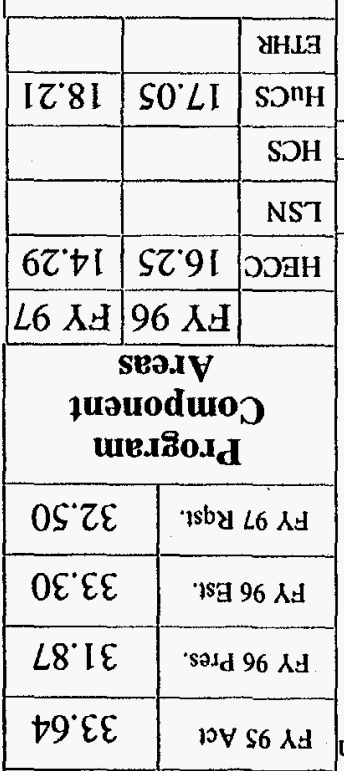

(W \$) 1วรีpng

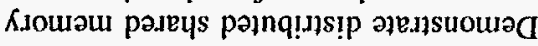

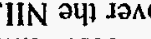

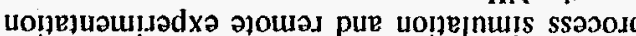

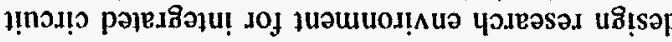

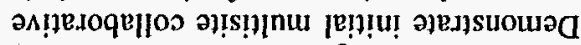

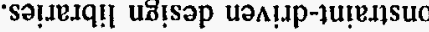

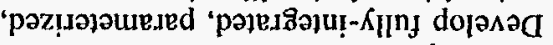

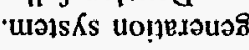

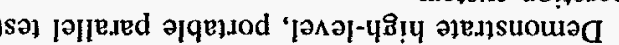

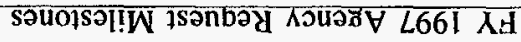

'suo!neısyrom jo dolsnjo uo sjuəuoduoo

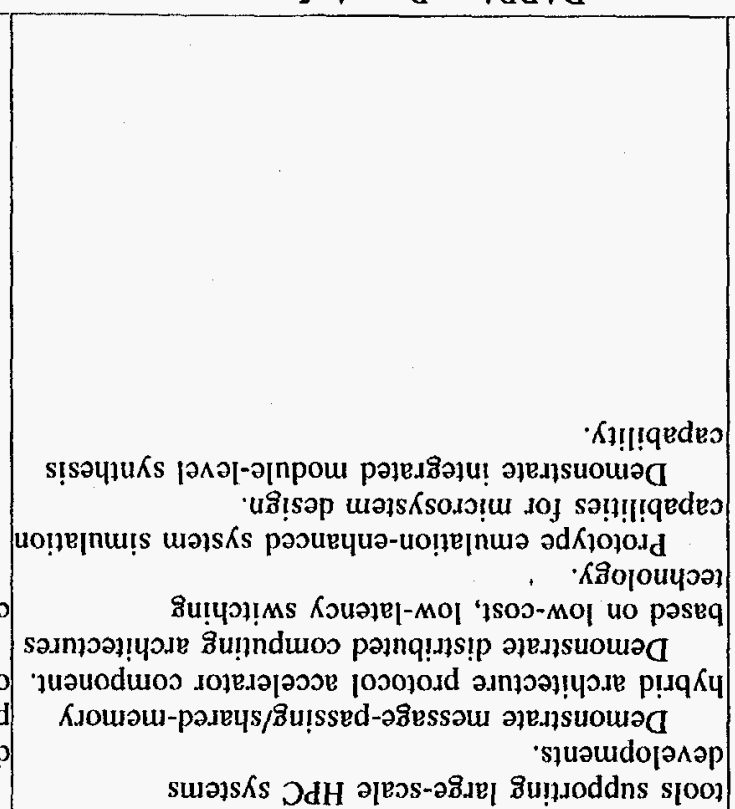

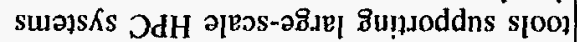

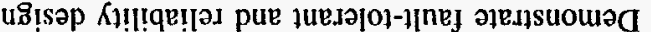

s)!noj!̣ je!nuonbos pue jвuo!jeu!quos ypoq
JoJ uo!̣viauos iso $\mathrm{L}$ oṇeuoin

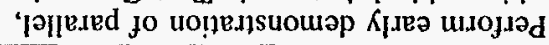

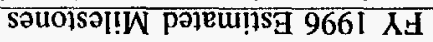

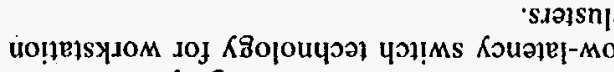

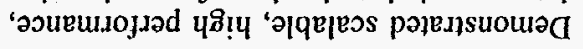

uo!̣es! [da.

jo s)!un әโqe Ioj sammoa!! suelsks gluonoaja dof luavuodinu

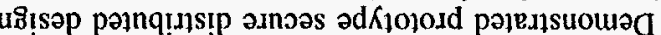
spoypau su!d 1 1010.10

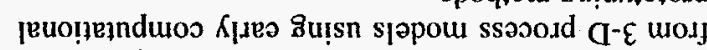

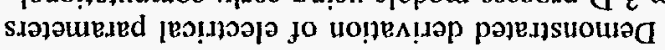

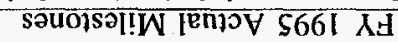

saduey

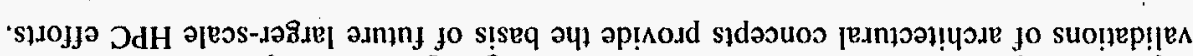

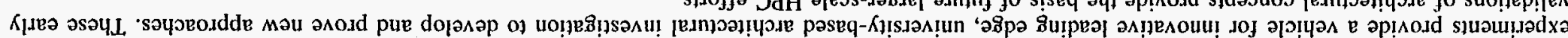

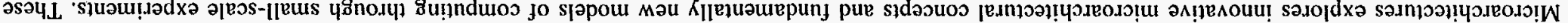

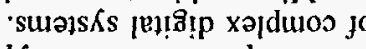

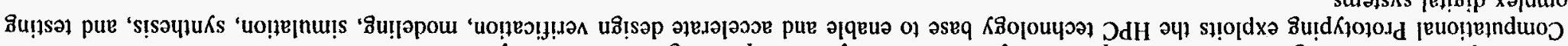

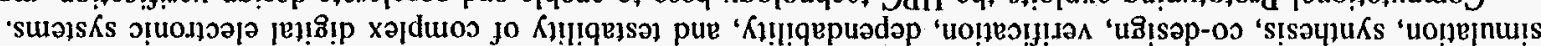

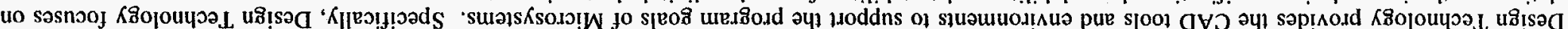

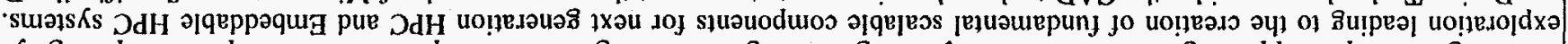

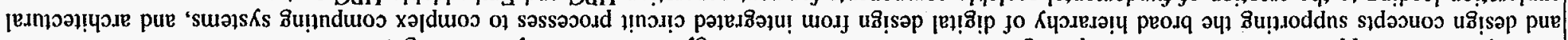

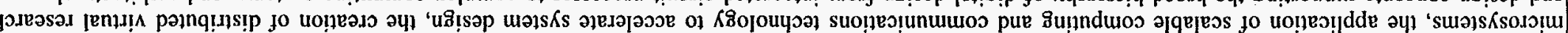

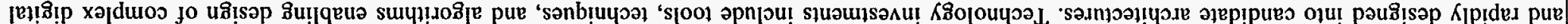

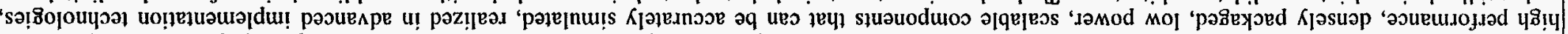

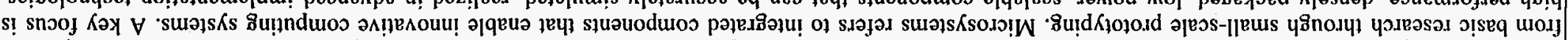

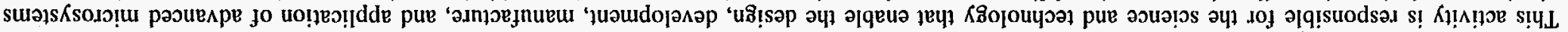


The Systems Environments Program supports the development of advanced software technologies needed to enable and accelerate the development, $\mathrm{N}$ introduction, and effective use of scalable high performance computing technologies. It is motivated by the need for a stable scalable software environment to enable defense mission agencies to leverage scalable systems and preserve their software investments across multiple generations of computing systems. The Systems Environments Program seeks to address this problem in each of three areas: Languages and Runtime Services, Scalable Software Library Technology, and Experimental Applications.

Languages and Runtime Services focuses on the technologies to develop high performance, cross architecture services for application programs. The project is coordinating three aspects: minimal extensions to well-known programming languages, compiler technologies that automate exploitation of concurrency and data locality, and advanced languages such as ORCA and SISAL in which concurrency is explicit.

Scalable Software Library Technology develops a suite of compatible library routines for major classes of HPC architectures and for major application areas. This enables application writers to simply link in the appropriate library for each class of machine. This project's initial focus is on demonstration of application portability and high performance using libraries that contain versions of major algorithms for several architectures.

Experimental Applications develops scalable versions of defense critical applications leveraging the HPC environments developed by the Languages and Runtime Services project and Scalable Software Library Technology components. The Experimental Applications component is coordinated with other

DARPA offices, the Defense HPC Modernization office, other defense agencies, and other government agencies, as appropriate.

Budget (\$ M)

\begin{tabular}{|c|c|}
\hline FY 95 Act & 35.15 \\
\hline FY 96 Pres. & 24.62 \\
\hline FY 96 Est. & 21.96 \\
\hline FY 97 Rqst. & 17.68 \\
\hline
\end{tabular}

Program

Component

Areas

FY $9 6 \longdiv { \text { FY } 9 7 }$

\begin{tabular}{|l|l|l|}
\hline HECC & 18.28 & 15.50 \\
\hline LSN & & \\
\hline HCS & & \\
\hline HuCS & 3.68 & 2.18 \\
\hline ETHR & & \\
\hline
\end{tabular}

Milestone

FY 96 milestone for scalable OS moved to Scalable Systems and Software

Changes
Demonstrated prototype integrated HPC programming environment for Fortran and $\mathrm{C}++$ on

which applications run transparently on several

distinct scalable computer architectures without change.

Completed detailed study of $1 / O$ characteristics of scalable computers under real application load, identifying significant bottlenecks.

Demonstrated tools for performance tuning of application software using dynamically-collected statistics.

FY 1996 Estimated Milestones

Evaluate small-scale teraops class systems and individual gigaops processors.

Define second generation of High Performance Fortran with extensions for task parallelism and support for scalable $\mathrm{I} / \mathrm{O}$.

Demonstrate extensions of portable scalable

libraries to incorporate object-oriented technology and a broader set of applications.

Enhance and experimentally cvaluate advanced software environment that supports composition tools for software creation, integration,

Demonstrated portable scalable software libraries development, and testing using animation across three major computer architectures applied to techniques.

semiconductors device simulation.
Demonstrate optimizing compilers with 5-to-10 times runtime performance improvement through partial compilation and late optimization during program execution.

Define High Performance $\mathrm{C}++$ with extensions for both Data Parallel and Task Parallel

exploitation of concurrency.

Prototype common runtime services reducing urden on individual compiler R\&D efforts.

Provide scalable versions of widely-used commercial engineering software, including MCS NASTRAN, leveraging scalable software library technology available to the defense community. Demonstrate feasibility of utilizing advanced software environment that supports composition tools for composing software, integration, and software development and testing using animation

\section{FY 1997 Agency Request Milestones} techniques in military environment.

\begin{tabular}{|c|l|}
\multicolumn{2}{|c|}{ Agency Ties } \\
\hline DARPA & \\
\hline NSF & Partner \\
\hline DOE & Partner \\
\hline NASA & Partner \\
\hline NIH & Partner \\
\hline NSA & Partner \\
\hline NIST & Partner \\
\hline NOAA & Partner \\
\hline EPA & Partner \\
\hline ED & \\
\hline AHCPR & \\
\hline VA & \\
\hline
\end{tabular}




\begin{tabular}{|c|c|c|l}
\hline DARPA & Networking Systems & Budget Code: & ST-19 \\
\hline This activity is developing high performance networking technologies to provide enabling technology in support of global climate simulation, high
\end{tabular}

This activity is developing high performance networking technologies to provide enabling technology in support of global climate simulation, high resolution remote imagery exploitation, telemedicine as well as national- and global-scale applications requiring high data-rate wide and local area communication and information services. This activity will produce networking technologies needed to enable the effective interconnection of the high performance computing systems produced by the other parts of the HPCC Program. The Internetworking activity develops technology in four areas: Internet Protocols, Communication Services, Routing and Scaling, and Network Security. Internet Protocols insure that differing types of network "bitways" work together as a cohesive system to build networks from a local to global scale and from slowest to fastest. Research in this area also insures that integration of the newest high performance technology continues to interwork with existing and slower technology in a systematic manner. Routing and Scaling technologies insure that networks continue to provide reliable services as the number of networks and connected endpoints continue to increase. Communication Services builds on the basic transmission capability of the underlying technology to provide additional services, such as multicast and guaranteed Quality of Service, to applications. Finally, the Network Security activity collaborates with the Defensive Information Warfare program to integrate security techniques into the evolving network infrastructure.

The High Performance Networking activity is concerned with satellite, broadcast communication, switching technology, telecommunications standards, optical transmission, and affordable local area designs. This activity develops technology to reduce latency and increase bandwidth by focusing on new techniques to interface computers and their applications to networks, to provide distributed computing services that enables high inter-application performance, and to evaluate and measure performance through continual experimental applications. The objective is to create a suite of complementary communication systems that can be integrated into an overall system with all of the strengths and none of the weaknesses of individual systems. Through the use of testbeds, a continually escalating level of performance for application development is provided while validating the effectiveness of the supporting technologies.

\section{Milestone Changed budget from HECC to LSN}

Changes

FY 1995 Actual Milestones

Demonstrated bandwidth, delay, and service reservation guarantees for networks in support of real-time control and critical services.

Demonstrated Synchronous Optical Network (SONET) and Asynchronous Transfer Mode (ATM) encryption technologies at $155 \mathrm{Mb} / \mathrm{s}$ (OC-3c). Deployed small-scale, initial prototype of gigabit-per-second class, nation-spanning infrastructure in support of high performance computing applications.

Demonstrated advanced network capabilities, including multicast-based services and next generation Internet protocols with improved ease of use.

\section{FY 1996 Estimated Milestones}

Demonstrate higher level communication services that coordinate distributed computing resources across the network environment. Prototype networks at greater than 40-gigabit-per-second speed using optical technologies and experimentally validate scalable network protocols at the higher speeds.

Prototype secure nomadic computing architecture integrated into existing wide area networks.

Deploy reference implementation of protocol-independent, multicast-capable infrastructure as basis for devclopment of advanced services.

Demonstrate robust and secure network-level infrastructure protocols to include directory services and resource allocation.

Demonstrate technology for autonomous, node-level network management.
FY 1997 Agency Request Milestones

Demonstrate transport protocols for mult gigabit networks.

Demonstrate systems for coordinating sets of workstations as a single computing system.

Deploy reference implementation of a common base set of network infrastructure protocols and services necessary for secure and reliable network operation.

Demonstrate wide-area 40-gigabit-per-secon and lab-prototype $100+$ gigabit-per-second electro-optical transmission and switching systems.

Develop advanced multicast-based services to include refinements of collaboration systems and autonomous network processes.
Budget (\$ M)

\begin{tabular}{|c|c|}
\hline FY 95 Act & 35.31 \\
\hline FY 96 Pres. & 31.43 \\
\hline FY 96 Est. & 33.72 \\
\hline FY 97 Rqst. & 33.76 \\
\hline \multicolumn{3}{|c|}{ Program } \\
Component \\
Areas
\end{tabular}

Agency Ties

\begin{tabular}{|c|l|}
\hline DARPA & \\
\hline NSF & Partner \\
\hline DOE & Partner \\
\hline NASA & Partner \\
\hline NIH & Partner \\
\hline NSA & Partner \\
\hline NIST & Partner \\
\hline NOAA & \\
\hline EPA & \\
\hline ED & \\
\hline AHCPR & \\
\hline VA & \\
\hline
\end{tabular}

December 20, 1996 
Advanced technologies are of value when they are integrated into usable systems in support of national defense capabilities. This activity is intended to $\$$ help transition advanced technologies into the defense mainstream by:

- Developing "middleware" adaptive services technology enabling interoperable secure transactions, resource discovery, information management, and survivable distributed computing:

- Integrating and demonstrating "middleware" services technology within the context of realistic large-scale systems;

- Leveraging commercial high performance computing hardware and software developments tailored for military applications;

- Enabling interoperability by establishing a building-block architecture common to many defense solutions.

The Embeddable Systems activity focuses on leveraging and extending the commercial scalable computing technology base to support present and future defense embedded computing applications. This activity makes strategic embedded-specific investments through leveraging advanced systems packaging technology to achieve system "smallness" with regard to power, weight, and size.

The Application Support Technology activity focuses on extending general commercial service technologies to support sophisticated

information-intensive applications developed for defense needs.

The Technology and Services Validation activity consists of focused efforts for fundamentally enabling applications, such as Digital Libraries and Electronic Commerce.

(continued)

Changes

FY 1995 Actual Milestones

Demonstrated use of advanced visualization

environment in a defense application.

Developed a set of communication benchmarks communication protocols, and prototype for

embedded, scalable military systems.

First Message-Passing Interface (MPI) demonstration of cross-architecture application portability.

Demonstrated integrated access to several

different special, classified defense and intelligence information systems.

Demonstrated 10 GigaFLOPS/cu.ft. militarized HPC System.

\section{FY 1996 Estimated Milestones}

Develop and provide experimental testbed services employing advanced high performance computing technologies for special defense users.

Prototype embedded computing system modules with scalability concepts containing memory hierarchy and power on a single unit of replication. Perform integration tests in key defense applications such as advanced distributed simulation, advanced distributed collaboration, advanced communications and control, and advanced human computer interfaces.

Demonstrate first fine-grained high performance embedded and scalable computer system.

Demonstrate graphical program environments for HPC embedded systems

Demonstrate prototype toolkits supporting development of applications adaptive to changes i the computing and communication environment.

Demonstrate prototype of information services through a testbed incorporating information management and secure transactions. (continued)

\section{FY 1997 Agency Request Milestones}

Complete the developments and transition of experimental testbed services employing high performance computing technologies to special defense users.

Demonstrate integrating testbed architecture ncorporating advanced distributed simulation, advanced distributed collaboration, advanced communications and control, and advanced human computer interfaces.

Demonstrate enhanced feature, real-time distributed operating systems for Embeddable HPC.

Demonstrate 100 gigaops $/ \mathrm{cu}$. ft. militarized

Demonstrate initial capabilities of intelligent information services architecture with multiple mechanisms for describing resource capabilities and with a uniform interface to hybrid search methods for resource retrieval.

Develop real-time image understanding algorithms for use in image registration, target recognition, and autonomous navigation for ground level and overhead reconnaissance and surveillance.
Budget (\$M)

\begin{tabular}{|c|c|}
\hline FY 95 Act & 22.35 \\
\hline FY 96 Pres. & 23.25 \\
\hline FY 96 Est. & 41.40 \\
\hline FY 97 Rqst. & 56.71 \\
\hline
\end{tabular}

Program

Component

Areas

FY 96 FY 97

\begin{tabular}{|l|l|l|}
\hline HECC & 8.20 & 14.00 \\
\hline
\end{tabular}

LSN

HCS

HuCS

ETHR

\begin{tabular}{l|l|l|}
24.19 & 37.47 \\
\hline
\end{tabular}

9.0

$01 \quad 5.24$

\section{Agency Ties}

\begin{tabular}{|c|c|}
\hline DARPA & \\
\hline NSF & Partner \\
\hline DOE & \\
\hline NASA & \\
\hline NIH & \\
\hline NSA & \\
\hline NIST & \\
\hline NOAA & \\
\hline EPA & \\
\hline ED & \\
\hline AHCPR & \\
\hline VA & \\
\hline
\end{tabular}

December 20, 1996 


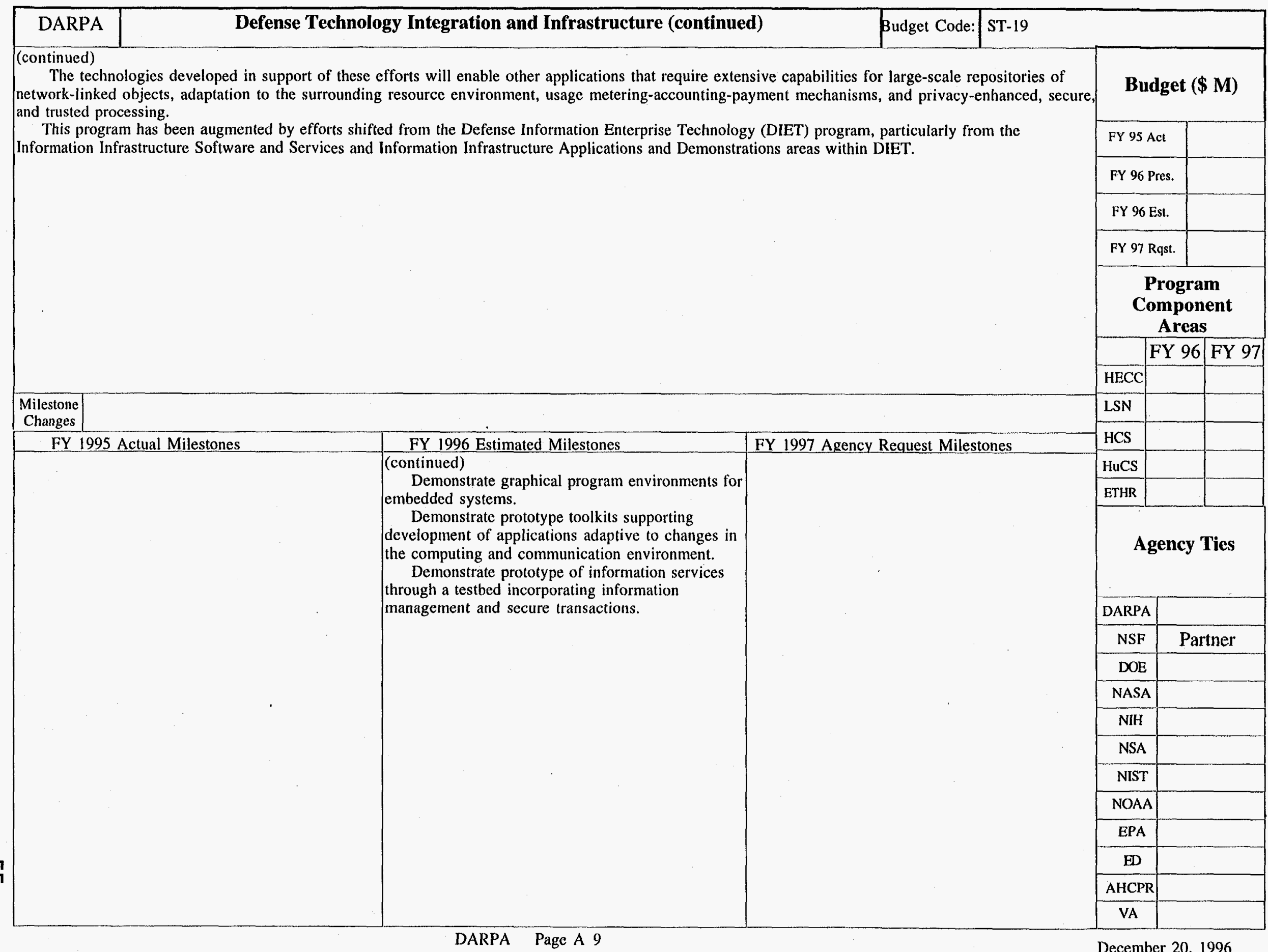




\begin{tabular}{|l|l|l|l|l|l|l|l} 
DARPA & Global Mobile Infosystems & Budget Code: & ST-19 \\
\hline
\end{tabular}

To satisfy defense requirements for rapidly deployable and robust information systems, it is critical that the technologies be available to support operation G) in mobile environments. This program will develop and demonstrate technologies to support this requirement. Design techniques, tools, languages, and environments, developed under the Microsystems program, will be extended to support design and prototyping of wireless computing systems. Architectures for modular untethered nodes will be developed and demonstrated that will leverage commercial advances in wireless technologies through the use of advanced design methodologies. New networking techniques will be developed and demonstrated that support rapid deployment and robust networking service in a hostile and dynamic environment. Advances in end-to-end networking technologies will be leveraged to provide highly capable communications services that can operate over a heterogeneous mix of networks including a variety of wireless networks. Finally, new computing paradigms will be developed that provide an underlying infrastructure supporting applications operation in an environment characterized by sporadic connectivity and varying quality of service.

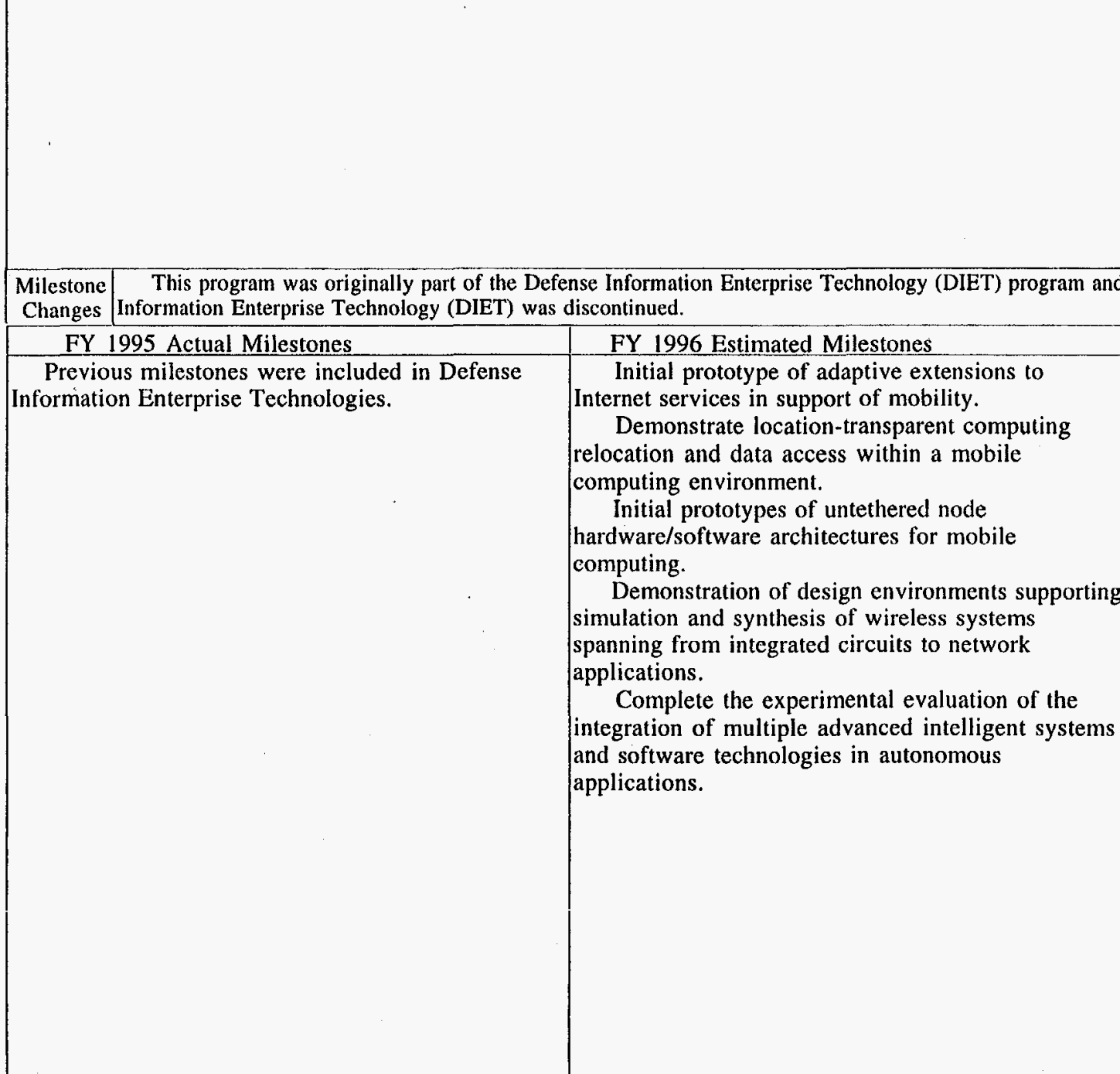

\section{Budget (\$ M)}

\begin{tabular}{|c|r|}
\hline FY 95 Act & 0.00 \\
\hline FY 96 Pres. & 0.00 \\
\hline FY 96 Est. & 16.30 \\
\hline FY 97 Rqst. & 17.58 \\
\hline
\end{tabular}

Program

Component

Areas

FY 96 FY 97

HECC

\begin{tabular}{|l|l|l|}
\hline LSN & 16.30 & 17.58 \\
\hline HCS & & \\
\hline HuCS & & \\
\hline ETHR & & \\
\hline
\end{tabular}

High performance CAD demo of 64 MBPS

CMOS wireless transceiver.

Demonstrate bandwidth-adaptive multimedia node for mobile computing.

Demonstrate advanced mobile networking

algorithms and protocols.

Demonstrate transparent application relocation within mobile environment.

\section{with}

imulation and synthesis of wireless systems

spanning from integrated circuits to network applications.

Complete the experimental evaluation of the ntegration of multiple advanced intelligent systems and software technologies in autonomous applications.
Agency Ties

\begin{tabular}{|c|c|}
\hline DARPA & \\
\hline NSF & \\
\hline DOE & \\
\hline NASA & \\
\hline NIH & \\
\hline NSA & \\
\hline NIST & \\
\hline NOAA & \\
\hline EPA & \\
\hline ED & \\
\hline AHCPR & \\
\hline VA & \\
\hline
\end{tabular}

December 20, 1996 


\begin{tabular}{|c|c|c|c|}
\hline DARPA & Information Survivability & Budget Code: & ST-24 \\
\hline
\end{tabular}

The Information Survivability activity develops the technology base underlying the solutions to protect DoD's mission-critical information systems against attack upon or through the supporting infrastructure. These technologies lead to generations of stronger protection, higher performance, and more cost-effective security solutions scalable to several thousand sites and to high performance computing technologies. The activity focuses on early prototypes of software and hardware technologies leading to scalable protection for large-scale heterogeneous systems usable over a wide range of performance in diverse threat environments. Technologies developed under this project will be exploited in other DARPA programs, including Networking Systems, Scalable Systems and Software, Microsystems, Defense Technology Integration and Infrastructure, Defense Information Enterprise Technologies, Intelligent Integration of Information, Advanced Distributed Simulation, Health Information Infrastructure, Optical Networking, Software Assurance, Software Foundations, Embedded Computing, and Global Mobile Communications, to satisfy defense requirements for secure and survivable systems. The activity is organized into four areas: High Confidence Networking, High Confidence Computing Systems, Assurance and Integration, and Survivability and Vulnerabilities.

High Confidence Networking technologies will be developed consisting of security mechanisms and value-added security services for integration into network technologies, as well as robust networking protocols that provide modular security services and mechanisms.

High Confidence Computing Systems includes secure and fault-tolerant operating systems, firewalls, and system management tools to provide high reliability for distributed computations and to allow geographically-separated parts of an organization to interact as if they shared a common security perimeter.

Assurance and Integration tools will allow the development of high assurance and trusted systems that add expression of modular system structures, networking, and other distributed-system protocols and the ability to reason about their security and robustness properties.

The Survivability and Vulnerabilities area studies other vulnerabilities in the defense computing infrastructure and in emerging critical technologies that could be exploited by an information warfare enemy.

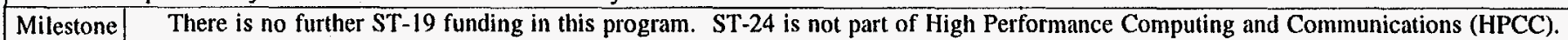

Changes

FY 1995 Actual Milestones

FY 1996 Estimated Milestones

Developed basic authentication and authorization This program was not part of HPCC in 1996. mechanisms based on digital signatures,

cryptography, and privacy-enhanced mail for use in a common infrastructure.

Completed proof-of-concept Asynchronous

Transfer Mode (ATM) encryption units for use in experimental ATM networks.

Began operation of certification authority

supporting privacy enhanced mail and other secure services.

Completed prototype implementation of digital signature hierarchy toolkit and domain name system enhancements.

Demonstrated operating system capability for strict process separation.

Demonstrated prototype signature/time stamp server with associated access tools for location-independent object security.

This program was not part of HPCC in 1996.

a

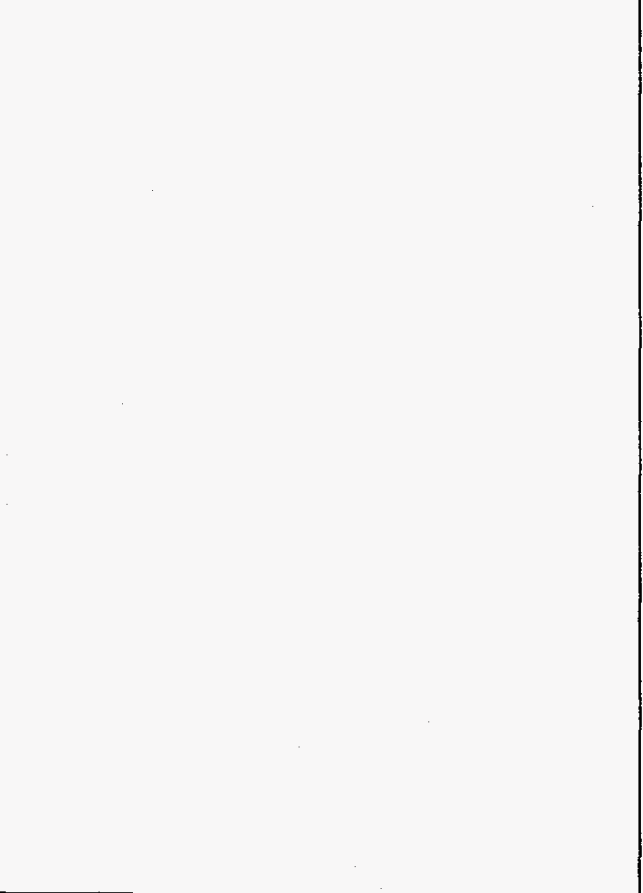

DARPA Page A 11
Budget (\$ M)

\begin{tabular}{|c|c|c|}
\hline \multicolumn{2}{|c|}{ FY $95 \mathrm{Act}$} & 9.88 \\
\hline \multicolumn{2}{|c|}{ FY 96 Pres. } & \\
\hline \multicolumn{2}{|c|}{ FY 96 Est. } & \\
\hline \multicolumn{3}{|c|}{ FY 97 Rqst. } \\
\hline \multicolumn{3}{|c|}{$\begin{array}{c}\text { Program } \\
\text { Component } \\
\text { Areas }\end{array}$} \\
\hline & FY 96 & FY 97 \\
\hline HECC & & \\
\hline LSN & & \\
\hline $\mathrm{HCS}$ & & \\
\hline $\mathrm{HuCS}$ & & \\
\hline ETHR & & \\
\hline
\end{tabular}

Agency Ties

\begin{tabular}{|c|l|}
\hline DARPA & \\
\hline NSF & Partner \\
\hline DOE & Partner \\
\hline NASA & \\
\hline NIH & \\
\hline NSA & Partner \\
\hline NIST & Partner \\
\hline NOAA & \\
\hline EPA & \\
\hline ED & \\
\hline AHCPR & \\
\hline VA & \\
\hline
\end{tabular}

December 20, 1996 
$\cup$ This activity will develop an intelligent, agent-based clinical associate software system and demonstrate its application in Ambulatory Care and Combat $\infty$ Casualty and Trauma Care. The program vision is to greatly improve the tools that support decision-making by clinical users and patients-consumers. The objectives of this activity are to:

(1) conduct visionary pilot projects of CLASS that demonstrate significant impact in health care; and

(2) develop a health care-specific information infrastructure that promotes use and reuse of component-based architectures for health information systems.

Models of the health care domain are constructed using a scenario-based engineering process. Domain-specific software architecture (DSSA) tools are used to derive reference architecture and requirements and to generate instances of user-based evaluation systems (visionary pilots). Important features and attributes of the sub-domain pilots are generalized to produce an associate systems reference architecture for health care (health care specific tools). The key functional components of this intelligent systems architecture include: (1) The human-computer interaction manager; (2) The task and context manage and (3) The information broker.

This architecture stresses multi-modal user interfaces; information integration from heterogeneous sources, multi-modal information acquisition, communication, and presentation, intelligent on-line support for medical personnel, logistical planning and resource allocation, and simulation-based systems for decision-makers in health care management. It provides a unique environment for developing tools and techniques for integrating and leveraging existing and emerging HPCC technologies. A key criterion for success is the transfer of the associate system and health care reference architectures into operational use in the Nation's industrial base.

\section{Milestone}

\section{Changes}

FY 1995 Actual Milestones

Developed domain models for ambulatory and trauma-combat casualty care.

Demonstrated CLASS -. Composite Health Care System mediator.

Piloted CLASS components in two clinic at National Naval Medical Center.

Developed CLASS reference architecture for ambulatory care.

Captured clinical data directly from physicians in two different clinics.

\section{FY 1996 Estimated Milestones}

Extend CLASS architecture to provide trauma guidelines directly to medics.

Integrate user-task models and knowledge-based decision support tools.

Demonstrate hands-free capture of patient data on battlefield.

Provide one-stop shopping for geographically dispersed human services clients.

Create reference architecture for generalized associate system.
FY 1997 Agency Request Milestones

Demonstrate protocol based care in all

battlefield and peacetime settings.

Facilitate transition of combat care associate to emergency services.

Initiate a new program in Learning

Technologies that will combine human centered information systems research with research in education to provide the basis for the next generation of technologies for education.

Demonstrate performance gains of advanced software engineering collaborators.
Budget (\$ M)

\begin{tabular}{|c|r|}
\hline FY 95 Act & 9.11 \\
\hline FY 96 Pres. & 10.24 \\
\hline FY 96 Est. & 6.24 \\
\hline FY 97 Rqst. & 7.51 \\
\hline
\end{tabular}

Program

Component

Areas

FY 96 FY 97

\begin{tabular}{|l|l|l|}
\hline HECC & & \\
\hline LSN & & \\
\hline HCS & & \\
\hline HuCS & & \\
\hline ETHR & 6.24 & 7.51 \\
\hline
\end{tabular}

Agency Ties

\begin{tabular}{|c|c|}
\hline DARPA & \\
\hline NSF & \\
\hline DOE & \\
\hline NASA & \\
\hline NIH & Partner \\
\hline NSA & \\
\hline NIST & \\
\hline NOAA & \\
\hline EPA & \\
\hline ED & \\
\hline AHCPR & \\
\hline VA & \\
\hline
\end{tabular}

December 20, 1996 


\begin{tabular}{|l|l|l|l|l|l|}
\hline DARPA & Information Sciences & Budget Code: & CCS-02 \\
\hline
\end{tabular}

The HPCC Information Sciences activity leverages basic software technology research supporting military, mission-oriented needs. This software technology research develops advanced concepts for methods and tools to produce high assurance software, language concepts that facilitate the rapid specification and evolution of software intensive defense systems, and techniques to manage shared complex-structured data objects in larger heterogeneous, distributed information systems.

Intelligent systems technology focuses on advanced techniques for knowledge representation, reasoning, and machine learning to enable computer understanding of spoken and written language and to advance methods for planning, scheduling, and resource allocation.

Human-computer interaction technology focuses on design methods and enabling technology for more natural interaction between people and computers.

Microelectronic science calibrates fundamental concepts to produce reliable, testable, and high performance design.

High Performance Computing (HPC) science generates concepts and methods for validating and verifying design components, and unique approaches to rapidly develop high performance libraries across multiple HPC architectures.

\section{Milestone}

\section{FY 1995 Actual Milestones}

Experimentally evaluated advanced information processing methods in spoken language

understanding, written language understanding, and automated planning systems.

Developed initial tool kits for interactive,

dialogue-based human computer interaction and

demonstrate them in a clinical environment.

Developed initial language-based methods for image understanding, high assurance, software

engineering system composition and experimentally evaluated process model approaches for prototyping large-scale software environments.

Experimentally evaluated library research that supports multiple parallel architectures.

Demonstrated health information network using South Florida Clinic.

Developed initial planning and decision aids prototypes for heterogeneous, distributed software system architectures and tools to support construction and maintenance of advanced intelligent systems. \begin{tabular}{l|c} 
FY 1996 Estimated Milestones & FY 1997 Agency Request Milestones \\
\hline Enhance benchmark problems, metrics, and test & Develop initial tools and tool kits for
\end{tabular} data sets and conduct experimental evaluations involving multiple intelligent systems and software engineering foundations technologies, utilizing knowledge acquisition.

Enhance advanced information processing methods in spoken \& written language understanding and automated planning systems.

Evaluate tool kits for interactive, dialogue-based uman computer interaction.

Evaluate language-based methods for image understanding, high assurance, and software environments system composition.

Begin experimental evaluation of design technology to include high performance computational prototyping of systems.

Evaluate planning and decision aids prototypes for heterogeneous, distributed software system architectures and tools to support construction and maintenance of advanced intelligent systems. development and evaluation of highly interactive agent and dialogue-based human computer interactions.

Experimentally support software evolution by integrating numerous formal and informal information sources in a "byperweb"; enhance formal notations for software design to include both syntactic and semantic information; and demonstrate multi-language architecture definition and analysis tools.

Advance the capabilities of spoken and written language understanding to solve real-world problems and provide widely usable human-computer interface functionality.

Extend and evaluate large-scale statistical modeling, machine learning, and knowledge representation methods for spoken and written language understanding and develop hub formalization that will infuse existing programming languages with new advances in formal methods.

Continue the experimental evaluation of design technology for high performance computational prototyping of systems.
Budget (\$M)

\begin{tabular}{|c|c|}
\hline FY 95 Act & 20.56 \\
\hline FY 96 Pres. & 23.72 \\
\hline FY 96 Est. & 23.10 \\
\hline FY 97 Rqst. & 23.35 \\
\hline
\end{tabular}

Program

Component

Areas

FY 96 FY 97

H

LSN

HCS

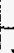

\begin{tabular}{|l|l|l|}
\hline HuCS & 20.50 & 19.26 \\
\hline ETHR & & \\
\hline
\end{tabular}

ETHR

Agency Ties

\begin{tabular}{|c|c|}
\hline DARPA & \\
\hline NSF & Partner \\
\hline DOE & \\
\hline NASA & \\
\hline NIH & \\
\hline NSA & \\
\hline NIST & \\
\hline NOAA & \\
\hline EPA & \\
\hline ED & \\
\hline AHCPR & \\
\hline VA & \\
\hline
\end{tabular}

December 20, 1996 
This activity leverages exploratory intelligent systems and software technology development that supports military, mission-oriented needs. It develops new information processing technology concepts that lead to fundamentally new software and intelligent systems capabilities. This will enable advanced information systems (involving both humans and computers) to more effectively accomplish decision-making tasks in stressful, time-sensitive situations and create efficient software systems supporting computer and software-intensive defense systems.

Major areas of technical emphasis are in:

(1) Intelligent systems (artificial intelligence), including autonomous systems, interactive problem solving, and intelligent integration of information from heterogeneous sources;

(2) Software development technology including languages, algorithms, data and object bases, domain specific software architectures, software prototype technology, software design tools, software reuse, and advanced software engineering environments; and

(3) Manufacturing automation and design engineering, including the development of advanced software systems that support sharing of engineering knowledge, advanced product and process design representations, integrated product and process design, software tools for design process management, manufacturing process planning, manufacturing process control and demonstrations.

Milestone

Changes

FY 1995 Actual Milestones

Evaluated the integration of advanced intelligent systems and technologies in multiple autonomous vehicles.

Upgraded the Image Understanding Environment and develop prototype implementations of advanced methods for vision guided navigation and cartographic modeling.

Developed initial prototype implementations for human-aided machine translation, document understanding, and robust speech understanding in adverse acoustic conditions.

Developed initial prototype implementations of advanced real-time planning and control algorithms. Enhanced knowledge based decision aids to support the rapid construction of multiple-crisis action plans.

Developed concepts and implementations of scalable machine intelligent algorithms for autonomous associate systems.

Developed initial prototype implementations of advanced methods for information fusion, aggregation, summarization, and explanation. (continued)

\section{FY 1996 Estimated Milestones}

Enhance test case scenarios and Internet accessible software testbeds.

Enhance and evaluate advanced methods for

vision guided navigation and cartographic modeling Evaluate implementations of real-time planning and control.

Evaluate knowledge-based decision aids.

Evaluate methods for information fusion,

aggregation, summarization, and explanation.

Evaluate scalable machine intelligent methods

for machine learning, automated reasoning and real time problem solving.

Enhance and evaluate advanced software environment that supports composition tool integration.

Enhance agent based architectures to include machine learning techniques and advanced information processing.

Demonstrate feasibility of authoring tools in creating domain-specific multimedia curricula.

Expand network design and manufacturing

services to include factory simulation and reusable product and process design libraries.

(continued)

\section{FY 1997 Agency Request Milestones}

Continue development of human-compute interaction, heterogeneous testbed products and insertion. Test, evaluate and demonstrate enhancements to the developer and user communities.

Develop modular Human Language Technologies to support easy, low-cost, rapid

technology transfer and application development for Document Understanding, Machine Translation, and Speech Understanding

Develop in the Intelligent Integration of Information area,tools and techniques to enable the rapid construction of information fusion, aggregation, and summarization software.

Demonstrate a software environment rapid construction facilities for robust software and intelligent systems technology prototypes.

Image understanding applications effort that will transition results in automatic target

recognition,terrain modeling for simulation, video surveillance, image database retrieval, and integrated reconnaissance and operations planning to other DoD agencies; continue multidisciplinary vision research with Office of Naval Research.

\section{Budget (\$ M)}

FY 95 Act

FY 96 Pres 77.34

FY 96 Est. 61.93

FY 97 Rqst. 72.14

Program

Component

Areas

FY 96 FY 97

HECC

LSN

HCS

HuCS

ETHR

61.93

72.14

Agency Ties

\begin{tabular}{|c|c|}
\hline DARPA & \\
\hline NSF & \\
\hline DOE & \\
\hline NASA & \\
\hline NIH & \\
\hline NSA & \\
\hline NIST & \\
\hline NOAA & \\
\hline EPA & \\
\hline ED & \\
\hline AHCPR & \\
\hline VA & \\
\hline
\end{tabular}

December 20, 1996 


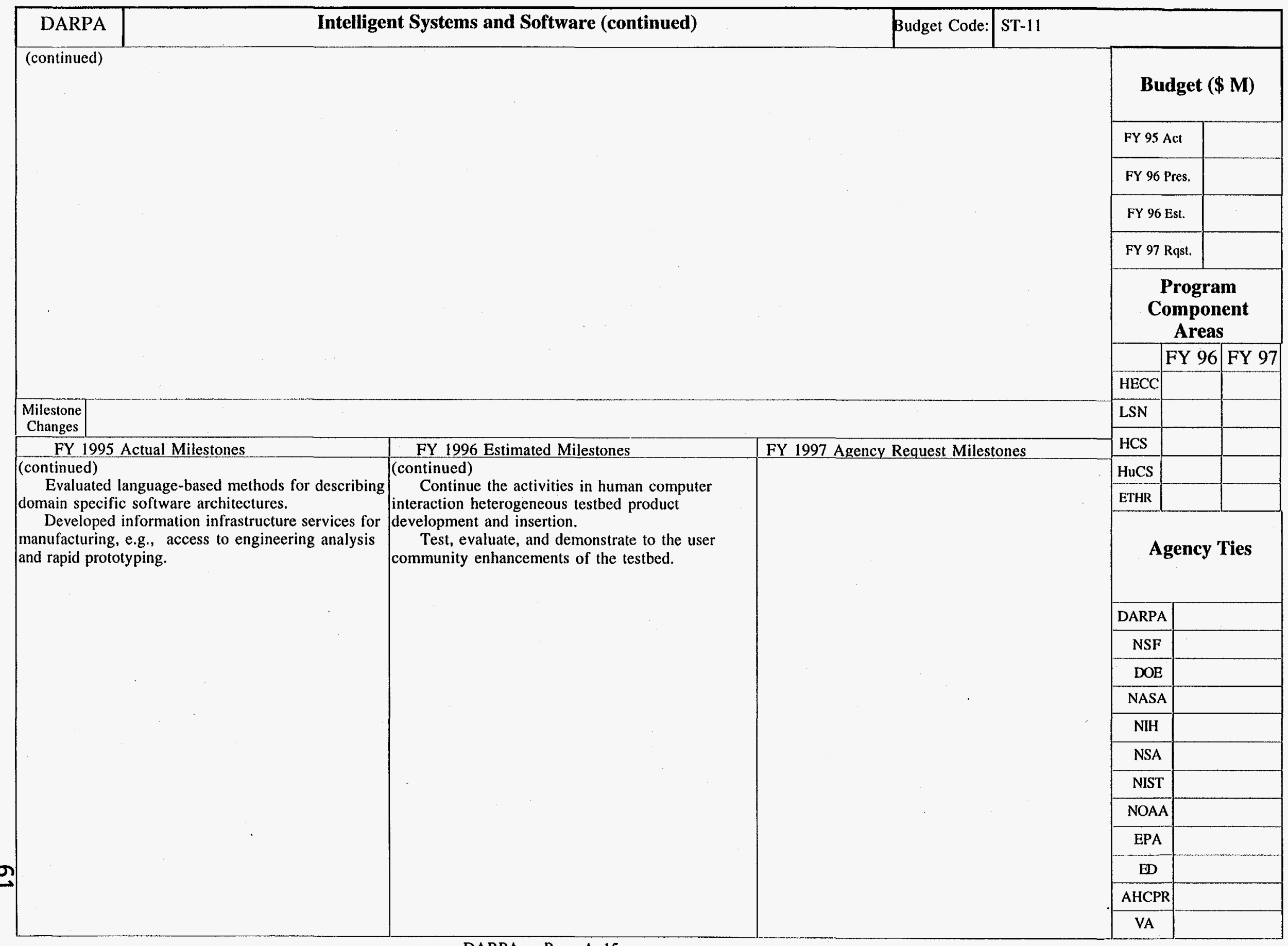


The objective of this activity is to develop and demonstrate key optical networking technologies with global reach meeting critical Department of Nofense needs by leveraging over commercial network developments.

The major emphasis of this activity is on optical Wavelength Division Multiplexing (WDM) network technologies. Optical fiber has a low loss transmission window of over $30 \mathrm{Thz}$, but today's network is only utilizing about $1 \%$ of this bandwidth. The transmission of data is limited to a single wavelength carrier and switching is done electronically. This activity will investigate the issues of multiple wavelength transmission and switching in both long-distance and local-access network topologies. To the extent possible, optical switching will be used to replace electronic switching to investigate the advantages of data-rate, format and protocol transparency. This activity supports device development, architecture, network control and management, testbeds and field trials.

A small part of this activity will investigate the technologies of ultra-fast optical Time-Division-Multiplexing. All-optical technologies will be emphasized, which includes switching, storage, logic gate, and switching and synchronization. The pulse width is in the picosecond range and the bandwidth is over 100 gigabits per second. These pulses are usually transmitted as solitons to eliminate the dispersive effect in optical fibers.

Milestone FY 97 milestone delete: integration with advanced optical testbeds; large scale planning demonstrations; and deployable JTF C3.

Changes Changed budget from HECC to LSN

FY 1995 Actual Milestones
Demonstrated liquid-crystal cross-connect for
high speed data routing.
high speed data routing.

Demonstrated network access module: 8 wavelengths, $2.5 \mathrm{~Gb} / \mathrm{s}$ per channel, that performs transmit, receive, add/drop, multiplex and demultiplex functions.

Demonstrated amplifier gain equalization with multiple wavelengths.

Completed long-distance WDM network

architecture analysis.

Completed candidate architecture for local exchange WDM network.

Completed initial phase of fiber link installation at ATDnet.

Completed target signaling parameters for WDM optical networks.

Demonstrated tunable receiver operation for a 32 wavelength transceiver set.

Demonstrated $100 \mathrm{~Gb} / \mathrm{s}, 375-\mathrm{km}$ soliton transmission.

FY 1996 Estimated Milestones
Demonstrate a WDM testbed at the San
Francisco Bay area.
Francisco Bay area.

Demonstrate $50 \mathrm{~Gb} / \mathrm{s}$ optical buffer.

Demonstrate initial operation of $40 \mathrm{~Gb} / \mathrm{s}$ cross-connect switch.

Complete local exchange traffic analysis.

Demonstrate WDM SONET/ATM self-protection ring network.

Complete phase I of network control \&

management software of local exchange network.

Demonstrate 32 wavelength tunable transmitter.

Demonstrate WDM ring network at San

Francisco Bay area.

\section{FY 1997 Agency Request Milestones}

Finalize network control and management strategy of WDM network.

Demonstrate WDM field trial at ATDnet.

Demonstrate final phase field trial at San

Francisco Bay area.
Budget (\$ M)

\begin{tabular}{|c|c|c|}
\hline \multicolumn{2}{|c|}{ FY 95 Act } & 21.79 \\
\hline \multicolumn{2}{|c|}{ FY 96 Pres. } & 26.83 \\
\hline \multicolumn{2}{|c|}{ FY 96 Est. } & 26.83 \\
\hline \multicolumn{2}{|c|}{ FY 97 Rqst. } & 32.12 \\
\hline \multicolumn{3}{|c|}{$\begin{array}{c}\text { Program } \\
\text { Component } \\
\text { Areas }\end{array}$} \\
\hline & FY 96 & FY 97 \\
\hline \multicolumn{3}{|l|}{$\mathrm{HECC}$} \\
\hline LSN & 26.83 & 32.12 \\
\hline \multicolumn{3}{|l|}{$\mathrm{HCS}$} \\
\hline \multicolumn{3}{|l|}{ HuCS } \\
\hline ETHR & & \\
\hline
\end{tabular}

\section{Agency Ties}

\begin{tabular}{|c|c|}
\hline DARPA & \\
\hline NSF & \\
\hline DOE & \\
\hline NASA & \\
\hline NIH & \\
\hline NSA & Partner \\
\hline NIST & \\
\hline NOAA & \\
\hline EPA & \\
\hline ED & \\
\hline AHCPR & \\
\hline VA & \\
\hline
\end{tabular}

December 20, 1996 


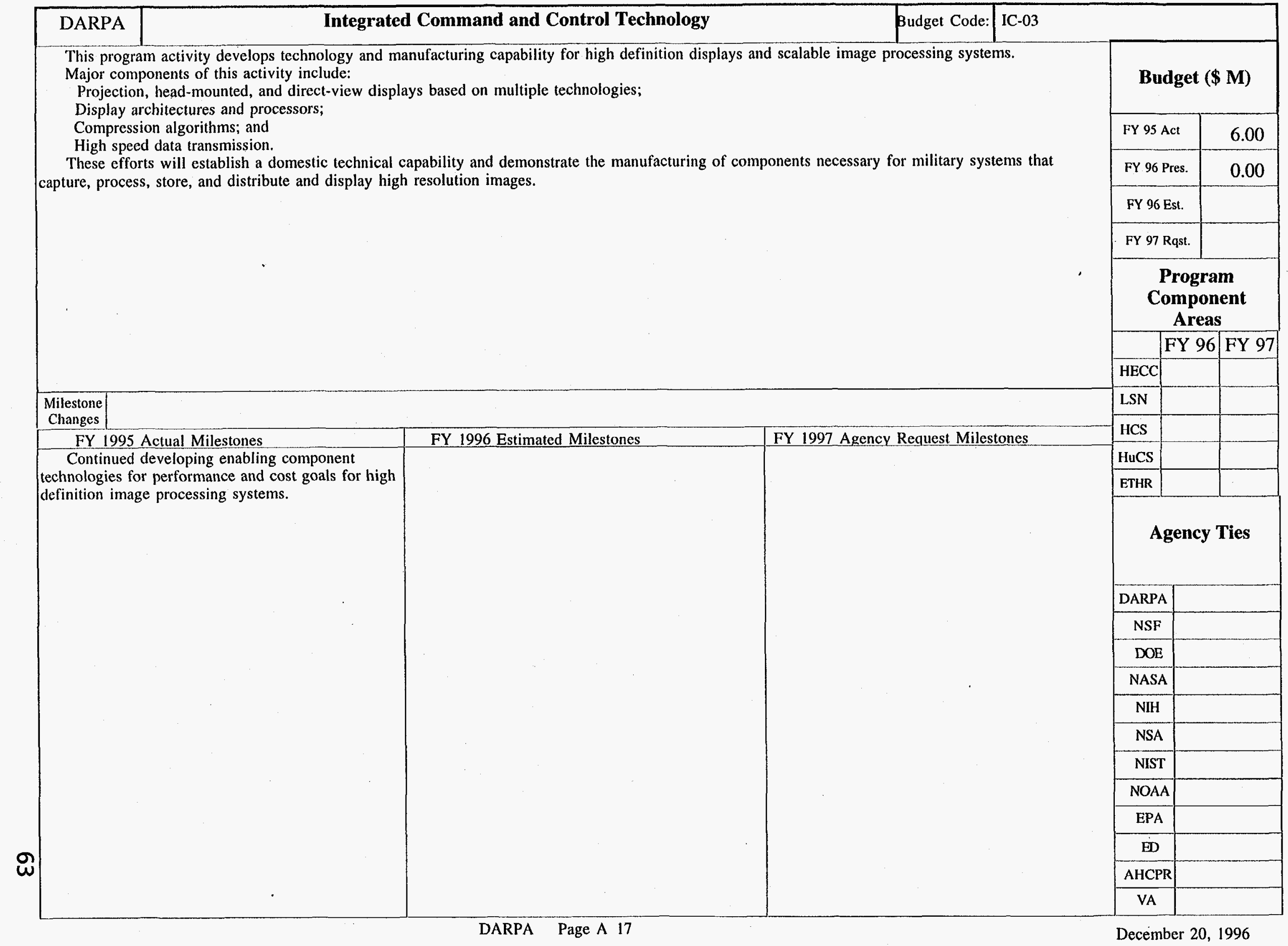




\begin{tabular}{|c|c|c|c|}
\hline DARPA & Defense Information Enterprise Technologies & Budget Code: & ST-19 \\
\hline
\end{tabular}

This activity develops the underlying computer systems technology to enable applications developers to demonstrate prototype solutions to national- and global-scale problems. The technology is based on those developed for High Performance Computing and Communications, but exhibiting more extensive capabilities for ubiquitous information access, dynamic discovery of and adaptation to new resources, linkage of objects and their dissemination and distribution over inter networked distributed systems, and trusted system operation on behalf of users and applications. To drive the development of these technologies, this activity supports integrated systems technology projects. In particular, this activity will develop the underlying technology base for the fundamental enabling applications of Digital Libraries and Electronic Commerce.

The first element is developing service technologies available to applications developers, built upon bitways, that provides a ubiquitously available, network-aware, adaptive interface upon which to construct national- and global-scale applications. Information Infrastructure Applications Demonstrations are focused efforts for fundamentally enabling applications, such as Digital Libraries and Electronic Commerce. The technologies developed in support of these will enable other applications that require extensive capabilities for large-scale repositories of network-linked objects, adaptation to the surrounding resource environment, usage metering-accounting-payment mechanisms, and privacy-enhanced, secure, and trusted processing. The Global Mobile Information Systems activity is developing the technologies to support applications that must operate in the presence of platform mobility, sporadic connectivity, and dynamically varying bandwidths. Experimental services support experimental design for computational and physical prototyping.

Milestone This program was eliminated in FY 1996. Some efforts were moved to the Defense Technology Integration and Infrastructure program, and the Global Mobile Changes Infosystem subprogram was continued as a stand-alone program.

\begin{tabular}{|l|l|}
\hline FY 1995 Actual Milestones & FY 1996 Estimated Milestones \\
\hline Developed initial prototype of common & \\
authentication, authorization, and accounting \\
services infrastructure based on security mechanisms \\
in Defensive Information Warfare (ST-24) program. \\
Demonstrated prototypes of distributed digital \\
library technology including techniques for scalable \\
storage management and data repositories, persistent \\
object bases, and multimedia objects. \\
Demonstrated copyright management system, \\
providing proof of concept including fully electronic \\
copyright registration, recordation, rights transfer, \\
and management. \\
Demonstrated mobile computing system \\
Computer Aided Design (CAD) environment through \\
the design of early prototype, high bandwidth, \\
pico-cellular, and wireless access points to the \\
wireline infrastructure. \\
Demonstrated network-based access to Multichip \\
Module fabrication services.
\end{tabular}
FY 1997 Agency Request Milestones

Budget (\$ M)

FY 95 Act 29.61

FY 96 Pres. 57.61

FY 96 Est.

FY 97 Rqst.

Program

Component

Areas

FY $9 6 \longdiv { \text { FY } 9 7 }$

HECC

LSN HCS HuCS ETHR

Agency Ties

\begin{tabular}{|c|c|}
\hline DARPA & \\
\hline NSF & Partner \\
\hline DOE & \\
\hline NASA & Partner \\
\hline NIH & \\
\hline NSA & \\
\hline NIST & Partner \\
\hline NOAA & \\
\hline EPA & \\
\hline ED & \\
\hline AHCPR & \\
\hline VA & \\
\hline
\end{tabular}

December 20, 1996 


\section{National Science Foundation}

NSF supports and elaborates upon the Federal HPCC Program goals of extending U.S. technological leadership in high performance computing and communications, accelerating wide dissemination and application of technologies to speed the pace of innovation and to serve the national interests in many critical areas, and spurring gains in U.S. productivity and industrial competitiveness through the use of high performance computing and networking technologies. Program objectives, as the term is used here, refers to more specific ends, the attainment of which signals a major step toward achieving programmatic goals. For NSF, the objectives include:

Developing national research and education networking services and capabilities for connecting universities, high schools, research laboratories, libraries, and businesses at speeds of up to one billion bits per second;

Providing early access to new generations of scalable parallel high performance computers and software technologies in order to achieve performance of one trillion computer calculations per second on application areas representing Grand Challenges;

Generating fundamental knowledge with the potential for radically changing the state of high performance computing and communications;

Creating a cadre of scientists, engineers, and technical personnel knowledgeable in the ideas, methods, and value of computational science and engineering and prepared to take advantage of these new capabilities;

Encouraging industrial partnerships and affiliations to enhance innovation, technology transfer and U.S. productivity and industrial competitiveness;

Making advanced computing and communications information infrastructure available to a larger segment of the society to solve information intensive National Challenges and advance education.

The NSF strategy for meeting its goals and objectives consists of balanced programs of support for:

Individual investigators performing long-term curiosity-driven research;

Small group research teams studying single, broader problems;

Grand Challenge Applications Groups working on complex problems requiring multidisciplinary teams;

Science and Technology Centers, targeted on major and significant research areas;

Deployment of infrastructure, including general availability of networking services, access to specialized high performance computing capabilities, and provision of local small-scale state-of-art computing instrumentation; and

Developing new opportunities and technologies for enhancing science and engineering education.

For FY 1997, NSF has restructured its program into activities that reflect recommendations incorporated in reports such as the CIC Strategic Implementation Plan 'America in the Age of Information' and the National Research Council report 'Evolving the High Performance Computing and Communications Initiative to Support the Nation's Information Infrastructure.' This restructuring reflects both the Strategic Focus Areas of the CIC plan and the structure of the NSF. The new organization of the program is reflected in the following seven activity sheets; the original seventeen activities are included to capture past accomplishments and to help elucidate the transition. 


\section{National Science Foundation}

\begin{tabular}{|c|c|c|c|c|c|c|c|c|c|c|c|}
\hline \multirow[b]{2}{*}{ Program Activity } & \multirow[b]{2}{*}{ Budget Account Code } & \multirow{2}{*}{$\begin{array}{c}\text { Partner/User } \\
\text { Agencies }\end{array}$} & \multicolumn{4}{|c|}{ Budget $(\mathrm{BA}, \$ \mathrm{M})$} & \multicolumn{5}{|c|}{ HPCC PCAs by 1997 Pres. Request } \\
\hline & & & $\begin{array}{l}\text { FY } 95 \\
\text { Actual }\end{array}$ & $\begin{array}{c}\text { FY } 96 \\
\text { Pres. }\end{array}$ & \begin{tabular}{c|c|} 
FY 96 \\
Est.
\end{tabular} & $\begin{array}{c}\text { FY } 97 \\
\text { Rqs. }\end{array}$ & HECC & LSN & HCS & HuCs & ETHR \\
\hline NSFNET & & $\begin{array}{l}\text { All HPCC } \\
\text { Asencies }\end{array}$ & 44.18 & 46.22 & 44.04 & 41.64 & & 41.64 & & & \\
\hline Supercomputer Centers & & $\begin{array}{l}\text { DARPA, } \\
\text { NASA_NH }\end{array}$ & 70.91 & 74.90 & 69.36 & 57.73 & 57.73 & & & & \\
\hline Computing Systems & & & & & & 47.16 & 45.95 & & 1.21 & & \\
\hline Human Centered Systems & & & & & & 48.10 & & & & 48.10 & \\
\hline $\begin{array}{l}\text { Networking, Communications and the Convergence of } \\
\text { Computine \& Comm }\end{array}$ & & $\begin{array}{l}\text { DARPA,DOE,N } \\
\text { ASA }\end{array}$ & & & & 19.92 & & 19.92 & & & \\
\hline Applications & & DARPA,NASA, & & & & 55.21 & 25.49 & 8.20 & & 9.66 & 11.86 \\
\hline Education and Training & & DARPA,NASA,. & 20.66 & 20.24 & 16.62 & 9.75 & & 2.50 & & & 7.25 \\
\hline Research Centers & & & 10.68 & 11.08 & 11.38 & & & & & & \\
\hline Research Infrastructure & & & 19.23 & 21.59 & 18.20 & & & & & & \\
\hline Grand Challenge Applications Groups & & $\widehat{A R P A}$, EPA & 12.83 & 7.25 & 7.32 & & & & & & \\
\hline Computing Systems and Components & & & 19.58 & 22.33 & 17.20 & & & & & & \\
\hline Software Systems and Algorithms & & & 29.82 & 29.01 & 26.66 & & & & & & \\
\hline Ubiquitous Computing and Communication & & $\begin{array}{l}\text { ARPA, DOE, } \\
\text { NASA }\end{array}$ & 10.52 & 14.80 & 14.44 & & & & & & \\
\hline Human-Machine Interaction \& Information Access & & ARPA & 20.14 & 14.55 & 15.69 & & & & & & \\
\hline Biological Sciences (non-NC/GC) & & & 9.89 & 11.43 & 12.42 & & & & & & \\
\hline Engineering (non-NC/GC) & & & 19.81 & 3.98 & 4.73 & & & & & & \\
\hline Geosciences (non-NC/GC) & & NASA, NOAA & 4.28 & 3.59 & 4.66 & & & & & & \\
\hline Computational Mathematics (non-NC/GC) & & & 8.14 & 6.11 & 8.19 & & & & & & \\
\hline Physical Sciences (non-NC/GC) & & NIST & 10.68 & 7.09 & 9.50 & & & & & & \\
\hline Social, Behavioral \& Economic Sciences (non-NC/GC) & & & 3.10 & 2.14 & 2.14 & & & & & & \\
\hline National Challenges & & ARPA, NASA & 7.28 & 17.33 & 8.55 & & & & & & \\
\hline & & Totals: & 321.73 & 313.64 & 291.10 & 279.51 & 129.17 & 72.26 & 1.21 & 57.76 & 19.11 \\
\hline
\end{tabular}




\section{National Science Foundation}

\begin{tabular}{|c|c|c|c|c|c|c|c|c|c|c|c|c|c|c|}
\hline \multirow[b]{2}{*}{ Program Activity } & \multicolumn{4}{|c|}{ Budget $(\mathrm{BA}, \$ \mathrm{M})$} & \multicolumn{5}{|c|}{ HPCC PCAs by 1997 Pres. Request } & \multicolumn{5}{|c|}{ HPCC PCAs by 1996 Estimated } \\
\hline & $\begin{array}{l}\text { FY 95 } \\
\text { Actual }\end{array}$ & $\begin{array}{c}\text { FY } 96 \\
\text { Pres }\end{array}$ & $\begin{array}{c}\text { FY } 96 \\
\text { Est. }\end{array}$ & $\begin{array}{l}\text { FY } 97 \\
\text { Rast: }\end{array}$ & HECC & LSN & HCS & HuCS & ETHR & HECC & LSN & HCS & HuCS & ETHR \\
\hline NSFNET & 44.18 & 46.22 & 44.04 & $\overrightarrow{41.64}$ & & 41,64 & & & & & 44.04 & & & \\
\hline Supercomputer Centers & 70.91 & 74.90 & 69.36 & 57.73 & 57.73 & & & & & 69.36 & & & & \\
\hline Computing Systems & & & & 47.16 & 45.95 & & 1.21 & & & & & & & \\
\hline Human Centered Systems & & & & 48.10 & & & & 48.10 & & & & & & \\
\hline $\begin{array}{l}\text { Networking, Communications and the Convergence of } \\
\text { Comouline \& Comm }\end{array}$ & & & & 19.92 & & 19.92 & & & & & & & & \\
\hline Applications & & & & 55.21 & 25.49 & 8.20 & & 9.66 & 11.86 & & & & & \\
\hline Education and Training & 20.66 & 20.24 & 16.62 & 9.75 & & 2.50 & & & .7 .25 & & 2.50 & & & 14.12 \\
\hline Research Centers & 10.68 & 11.08 & 11.38 & & & & & & & 6.30 & 0.68 & & & $\overline{4.40}$ \\
\hline Research Infrastructure & 19.23 & 21.59 & 18.20 & & & & & & & 8.64 & 2.48 & & 4.78 & 2.30 \\
\hline Grand Challenge Applications Groups & 12.83 & 7.25 & 7.32 & & & & & & & 7.32 & & & & \\
\hline Computing Systems and Components & 19.58 & 22.33 & 17.20 & & & & & & & 17.20 & & & & \\
\hline Software Systems and Algorithms & 29.82 & 29.01 & 26.66 & & & & & & & 26.66 & & & & \\
\hline Ubiquitous Computing and Communication & 10.52 & 14.80 & 14.44 & & & & & & & & 14.44 & & & \\
\hline Human-Machine Interaction \& Information Access & 20.14 & 14.55 & 15.69 & & & & & & & & 5.29 & & 6.80 & 3.60 \\
\hline Biological Sciences (non-NC/GC) & 9.89 & 11.43 & 12.42 & & & & & & & & 12,42 & & & \\
\hline Engineering (non-NC/GC) & 19.81 & 3.98 & 4.73 & & & & & & & 1.65 & 1.38 & & 1.70 & \\
\hline Geosciences (non-NC/GC) & 4.28 & 3.59 & 4.66 & & & & & & & 3.19 & 1.47 & & & \\
\hline Computational Mathematics (non-NC/GC) & 8.14 & 6.11 & 8.19 & & & & & & & & 2.67 & & 2.00 & 3.52 \\
\hline Physical Sciences (non-NC/GC) & 10.68 & 7.09 & 9.50 & & & & & & & & 6.41 & & & 3.09 \\
\hline Social, Behavioral \& Economic Sciences (non-NC/GC & 3.10 & 2.14 & 2.14 & & & & & & & & 2.14 & & & \\
\hline National Challenges & 7.28 & 17.33 & 8.55 & & & & & & & & 8.55 & & & \\
\hline Totals: & 321.73 & 313.64 & 291.10 & 279.51 & 129.17 & 72.26 & 1.21 & 57.76 & 19.11 & 140.32 & 104.47 & & 15.28 & 31.03 \\
\hline
\end{tabular}




\begin{tabular}{|c|c|c|c|}
\hline NSF & NSFNET & Budget Code: \\
\hline
\end{tabular}

9 The purpose of the NSFNET program activity is to provide for the high performance data networking needs of the U.S. research and education community $\infty$ This program activity supports operation of the worldwide Internet, and also funds enhancements to and participation in it.

More than 1,100 U.S. colleges and universities have been connected to the Internet through the NSFNET program activity, and several thousand high schools have had their connection facilitated. Libraries, medical schools, and public health facilities have also been connected. The program activity has directly stimulated the emergence of a vigorous and highly competitive private-sector industry in Internet hardware, software, and connectivity in which the U.S. is a world leader with an overwhelmingly positive balance of trade.

A new, quasi-operational very high speed network backbone service (the 'vBNS') links NSF-supported high performance computing centers, and links are being established competitively to scientific applications that demand the performance available. For less demanding, general-purpose networking needs of the community, this activity supplies funds to regional networks --most of which have their roots in regional university consortia, and, under the Connections Program, to individual academic institutions needing Internet connectivity. Together with networking programs in other Federal agencies, the NSFNET program activity participates in funding administrative functions of the Internet, and collaborates in provisioning international Internet links. The NSFNET activity also supports technical development in such areas as database access and bibliographic protocols, routing and addressing, security and privacy, and network management.

\section{Milestone
Changes}

\section{FY 1995 Actual Milestones}

Completed transition to new NSFNET architecture including: ANS;

Overseeing Merit's audit of subcontract award to Closing out Merit cooperative agreement for old NSFNET architecture.

Completed initiation of award for vBNS.

Began vBNS to NSF supercomputer centers at U $155 \mathrm{Mb} / \mathrm{s}$.

Connected vBNS to four NAPs at $>45 \mathrm{Mb} / \mathrm{s}$.

Issued solicitations:

for $v B N S$;

for connections to qualifying high-bandwidth applications; and

for additional International Internet Services. Made targeted awards in the network theory

initiative.

Supported connection of 50 additional institutions to the Internet.

Made two awards in vBNS Connections Program.

\section{FY 1996 Estimated Milestones}

Continue to expand connection of qualifying scientific applications to the vBNS.

Participate with other Federal agencies and the private sector in implementing new network-layer protocol on the Internet.

Support connection of 50 additional institutions to the Internet.

Pilot deployment of emerging privacy and security tools for the Internet.

\section{FY 1997 Agency Request Milestones}

As a result of ongoing privatization, reduce support of commodity-level network services to educational institutions, and increase support of very high bandwidth services which focus on experimental applications of high bandwidth networks and international networking.

Among the applications areas targeted for emphasis are distributed high performance computing, information based learning technologies, remote visualization and imaging, and tele-collaboration.

\section{Budget (\$ M)}

FY 95 Act

FY 96 Pres.

FY 96 Est. FY 97 Rgst. 41.64

Program

Component

Areas

\begin{tabular}{|l|l|l|}
\hline & FY 96 & FY 97 \\
\hline HECC & & \\
\hline LSN & 44.04 & 41.64 \\
\hline HCS & & \\
\hline HuCS & & \\
\hline ETHR & & \\
\hline
\end{tabular}

Agency Ties

\begin{tabular}{|c|c|}
\hline DARPA & Partner \\
\hline NSF & \\
\hline DOE & Partner \\
\hline NASA & Partner \\
\hline NIH & Partner \\
\hline NSA & User \\
\hline NIST & User \\
\hline NOAA & User \\
\hline EPA & User \\
\hline ED & User \\
\hline AHCPR & User \\
\hline VA & User \\
\hline
\end{tabular}

December 20, 1996 


\begin{tabular}{|c|c|c|}
\hline NSF & Supercomputer Centers & Budget Code: \\
\hline The four NSF Supercomputer Centers are:
\end{tabular}

The four NSF Supercomputer Centers are:

1. Cornell National Supercomputer Facility at Cornell University;

2. National Center for Supercomputing Applications at the University of Illinois;

3. Pittsburgh Supercomputing Center at Carnegie Mellon University, University of Pittsburgh,and Westinghouse;

4. San Diego Supercomputer Center at the University of California at San Diego.

They represent major activities that serve the computational needs of all NSF science and engineering disciplines by providing over 8,000 users from 49 States access to state-of-the-art high performance computing resources. Additional activities at the Centers include: information on and access to emerging technologies; software tools for high performance computing; support for Grand Challenge applications; and education, training, and outreach at all levels. The Centers have pioneered partnerships with the private sector working to introduce HPC technologies intonational industries to solve design and manufacturing problems. They are also involved in testing the next generation network capabilities, including developing tools for ease of network navigation. The strategic goals of the Centers are:

Provide access to state of the art equipment to academic community;

Research emerging technologies; interact with vendors;

Develop tools and software for all aspects of high performance computing and communications;

Lead in computational science applications (Grand Challenges);

Provide education and training in the use of HPC (vector \&scalable systems, workstation access);

Act as a resource for State and local Centers;

Provide educational outreach at all levels.

\section{Milestone FY 1997 will be a transition year from the existing program to the new Partnerships for Advanced Computational Infrastructure.}

\section{Changes}

FY 1995 Actual Milestones

Installed new systems and upgrades of current systems at Supercomputer Centers to more powerful parallel computing systems needed to support the academic computing community.

Enhanced metacenter activities, especially in the area of mass storage for the national file system being developed at the Centers.

Added additional Regional Alliances (4-6) to enhance computing and communications expertise of individuals in university, education, public and private sectors.

Hosted annual Supercomputing meeting in San Diego, with Center Directors as General Chair and Program Chair (5,000 attendees anticipated).

\section{FY 1996 Estimated Milestones} Renew Supercomputer Centers cooperative agreements.

Establish National Metacenter as a seamless national computing environment across all four NS Centers and other affiliates.

Establish parallel computing as production method in computational research.

Provide access to computer systems with capacity approaching a teraFLOP.

Support demonstration of National Challenge applications.
FY 1997 Agency Request Milestones Initiate the Partnerships for Advanced Computational Infrastructure in order to provide access to high performance computing for the

academic research community at a level of one to two orders of magnitude greater than that typically available at a major research university.
Budget (\$ M)

\begin{tabular}{|c|c|}
\hline FY 95 Act & 70.91 \\
\hline FY 96 Pres. & 74.90 \\
\hline FY 96 Est. & 69.36 \\
\hline FY 97 Rqst. & 57.73 \\
\hline
\end{tabular}

Program

Component

Areas

FY 96 FY 97

\begin{tabular}{|l|l|l|}
\hline HECC & 69.36 & 57.73 \\
\hline
\end{tabular}

LSN

HCS

HuCS

$$
\text { ETHR }
$$

\section{Agency Ties}

\begin{tabular}{|c|c|}
\hline DARPA & Partner \\
\hline NSF & \\
\hline DOE & \\
\hline NASA & Partner \\
\hline NIH & Partner \\
\hline NSA & \\
\hline NIST & \\
\hline NOAA & \\
\hline EPA & \\
\hline ED & \\
\hline AHCPR & \\
\hline VA & \\
\hline
\end{tabular}

December 20, 1996 
Computing Systems Research is concerned with developing a fundamental understanding of computing systems, including their design and

Zimplementation, and the evaluation of novel computing and information processing systems and architectures with respect to their design criteria, usually

involving high levels of performance. Computing Systems deals with computer architecture, hardware implementation, system software(compilers, operating systems), interconnection networks, storage and $1 / O$ architectures, and novel computing structures and technologies that hold the promise for radically new computer systems of the next century. A unifying 'systems' focus is the development and demonstration of balanced scalable, parallel systems that can gracefully scale across a wide range of underlying numbers of processor nodes and interconnection structures.

TECHNOLOGY: (1) design and evaluation of instruction set architectures and the organization of central processing units; (2) memory systems; (3) computer system interfaces to communications networks and other high speed peripherals; (4) multilevel storage structures; (5)interconnection structures among processors, memories, and input/output channels; (6)compilers; (7) operating systems; (8) parallel algorithms; (9) fault tolerant and redundant hardware structures; and (10) high-performance input/output systems.

FOUNDATIONS: Development of a fundamental understanding of architectural design,interconnection structures, computational complexity, programming language semantics, and models of computation, with current emphasis on parallel and distributed algorithms and systems.

EXPERIMENTAL: Design, construction, and evaluation of high performance computing systems.

INFRASTRUCTURE: Access to high performance networks and computing systems for teams of university researchers.

\section{Budget (\$ M)

\begin{tabular}{|l|l|}
\hline FY 95 Act & \\
\hline FY 96 Pres. & \\
\hline FY 96 Est. & \\
\hline FY 97 Rast. & 47.16 \\
\hline
\end{tabular}

Program

Component

Areas

\begin{tabular}{|l|l|l|}
\hline & FY 96 & FY 97 \\
\hline HECC & & 45.95 \\
\hline LSN & & \\
\hline HCS & & 1.21 \\
\hline HuCS & & \\
\hline ETHR & & \\
\hline
\end{tabular}

Milestone

Changes

FY 1995 Actual Milestones

These are reported under Computing Systems and Components and Software Systems and Algorithms for FY 95.

FY 1996 Estimated Milestones

These are reported under Computing Systems and Components and Software Systems and Algorithms for FY 96

\section{FY 1997 Agency Request Milestones}

Continue an initiative, begun in FY 1996, to explore problem-solving environments for computation in science, through a multi-disciplinary approach.

Begin a new initiative on improved methods for software engineering, making use of powerful, mathematically-based techniques for analysis and synthesis of software systems.

Begin a new, inter-disciplinary initiative to apply massively parallel computing using biological materials, including DNA.

Determine whether and how the understanding that enabled the VLSI revolution might reapplied to the design of electromechanical systems.

Continue support of research that can enable more effective compilers for both tightly coupled and loosely distributed parallel systems.

Enable rapid advances in design for nano-manufacturing by supporting research to determine how formal, automated design techniques can be utilized.

\begin{tabular}{|c|c|}
\hline \multicolumn{2}{|c|}{ Agency Ties } \\
\hline DARPA & \\
\hline NSF & \\
\hline DOE & \\
\hline NASA & \\
\hline NIH & \\
\hline NSA & \\
\hline NIST & \\
\hline NOAA & \\
\hline EPA & \\
\hline ED & \\
\hline AHCPR & \\
\hline VA & \\
\hline
\end{tabular}

December 20, 1996 


\section{NSF}

Human Centered Systems

Budget Code:

Human-centered Systems research is concerned with improving the interactions among humans, computing systems, and information resources. Among the research issues addressed are data capture; information store, management and access;knowledge representation, delivery and distribution; intelligent human and computer interfaces;group and organizational interactions; determination of usability and adaptability; and programming paradigms and software environments tailored to problem domains and task specifications. The key challenge in this research is how to harness new information technologies for the benefits of diverse end users.

TECHNOLOGY: (1) Intelligent sensors and input/output devices; (2) Database and knowledge processing technology for data capture and store, knowledge acquisition and representation, information management and retrieval, and knowledge mining; (3) Human-system interfaces, including speech recognition, natural language understanding, and other modalities of human/machine communication; (4) Multi-media information technologies; (5)Machine learning technology, enabling the system to adapt its operations and interactions to human preferences; (6) Collaboration technology; (7) Virtual

environments, including both the advanced simulation and modeling technology and the virtual enterprise technology enabling the restructuring of businesses and corporations in the distributed workplace; (8) End-user enhancement technology, including large-scale robotics and very small-scale, embedded systems.

FOUNDATIONS: Development of the fundamental theories and models required to understand basic aspects of human/computer interactions.

EXPERIMENTAL: Design, construction, and evaluation of systems to support human/computer interactions and to validate models and theories for that interaction.

INFRASTRUCTURE: Access to high performance networks and computing systems for teams of university researchers and development of sharable data resources to support experimental work.

\section{Milestone \\ Changes}

FY 1995 Actual Milestones

These are reported under Human-Machine Interaction and Information Access.
FY 1996 Estimated Milestones These are reported under Human-Machine Interaction and Information Access.
FY 1997 Agency Request Milestones

Continue the Digital Library projects, a joint research initiative with DARPA and NASA, and support related new work in very large databases and knowledge repositories.

As part of a long-term goal to enable better and more facile access to information and computing resources by ordinary citizens emphasize research including virtual environments, multi-modal human-computer communications and human-language technology. Increase support for research in information-based learning technologies with the potential to transform education at all levels in the 21 st century and form a new enabler for the integration of research and education.

Initiate the multi-agency program,

STIMULATE: Speech, Text, Image, and

MUltimedia Advanced Technology Effort, in orde to understand multimodal human communication and apply it to computer technology.

\section{Budget (\$ M)}

\begin{tabular}{|l|l|}
\hline FY 95 Act & \\
\hline FY 96 Pres. & \\
\hline FY 96 Est. & \\
\hline FY 97 Rqst. & 48.10 \\
\hline
\end{tabular}

Program

Component

Areas

FY 96 FY 97

\begin{tabular}{|l|l|l|}
\hline HECC & & \\
\hline LSN & & \\
\hline HCS & & \\
\hline HuCS & & 48.10 \\
\hline ETHR & & \\
\hline
\end{tabular}

\section{Agency Ties}

\begin{tabular}{|c|c|}
\hline DARPA & Partner \\
\hline NSF & \\
\hline DOE & \\
\hline NASA & \\
\hline NIH & \\
\hline NSA & \\
\hline NIST & \\
\hline NOAA & \\
\hline EPA & \\
\hline ED & \\
\hline AHCPR & \\
\hline VA & \\
\hline
\end{tabular}

December 20, 1996 


\begin{tabular}{|c|c|c|c|c|c|c|c|}
\hline NSF & \multicolumn{3}{|c|}{ Networking, Communications and the Convergence of Computing \&Comm. } & Budget Code: & & & \\
\hline \multirow{8}{*}{\multicolumn{5}{|c|}{$\begin{array}{l}\text { The overall goal is to facilitate access to information and computing in order to effectively and efficiently overcome distance and time barriers. } \\
\text { Networking research focuses on architecture, protocols, and performance of different types of networks including high band-width networks with multiple } \\
\text { qualities of service guarantees, as well as wireless and all-optical networks. Communications research focuses on improving communication over optical and } \\
\text { electromagnetic channels and the systems that enable that communication and on developing new approaches to digital storage systems. It is increasingly } \\
\text { important to explore communications, computing, and networking as a single system. This convergence is emerging as computers become more universal } \\
\text { and integrated parts of networked environments, as communication becomes mostly digital, as distributed databases become networked, as the demand for } \\
\text { interactive and on-demand multimedia services increases, and as on-demand remote computing becomes available. } \\
\text { TECHNOLOGY: (1) Network access and control protocols; (2) Network management tools\& techniques; (3) Wireless networks; (4) Mobile computing; } \\
\text { (5) Optical systems; (6) Software to support distributed computing; (7) Software to support resource discovery and access to networked resources; (8) I/O } \\
\text { devices \& subsystems. } \\
\text { FOUNDATIONS: Theoretically based techniques for the design, specification, analysis,implementation, testing, maintenance, and modification of } \\
\text { architectures and protocols for networks and on-demand remote computing systems. } \\
\text { EXPERIMENTAL: Design, construction, and evaluation of networking, communications,and on-demand remote computing systems. } \\
\text { INFRASTRUCTURE: Multiple levels ranging from laboratory optical networks to wide area, complex, high speed networks; from wireless systems to } \\
\text { widely distributed databases and network storage devices, and networks of workstations to networks of supercomputers. The gigabit testbeds are examples of } \\
\text { infrastructure that supports research integrating communications, networking and computing. The vBNS is another example of infrastructure that will support } \\
\text { several classes of research activity. }\end{array}$}} & \multicolumn{3}{|c|}{ Budget $(\$ \mathbf{M})$} \\
\hline & & & & & \multicolumn{2}{|c|}{ FY 95 Act } & \\
\hline & & & & & \multicolumn{2}{|c|}{ FY 96 Pres. } & \\
\hline & & & & & \multicolumn{2}{|c|}{ FY 96 Est. } & \\
\hline & & & & & \multicolumn{2}{|c|}{ FY 97 Rqst. } & 19.92 \\
\hline & & & & & \multicolumn{3}{|c|}{$\begin{array}{l}\text { Program } \\
\text { Component } \\
\text { Areas }\end{array}$} \\
\hline & & & & & \multicolumn{2}{|c|}{ FY 96 } & FY 97 \\
\hline & & & & & \multicolumn{2}{|l|}{ HECC } & \\
\hline \begin{tabular}{|c|} 
Milestone \\
Changes
\end{tabular} & & & & . & LSN & & 19.92 \\
\hline FY 19 & Milestones & FY 1996 Estimated Milestones & FY 1997 & Request Milestones & HCS & & \\
\hline \multirow{15}{*}{\multicolumn{2}{|c|}{$\begin{array}{l}\text { These are reported under Software Systems and } \\
\text { Algorithms and Ubiquitous Computing and } \\
\text { Communication. }\end{array}$}} & \multirow{15}{*}{$\begin{array}{l}\text { These are reported under Software Systems and } \\
\text { Algorithms and Ubiquitous Computing and } \\
\text { Communication. }\end{array}$} & \multirow{15}{*}{\multicolumn{2}{|c|}{$\begin{array}{l}\text { Support software systems research to enable } \\
\text { the seamless convergence of computing and } \\
\text { communications leading to a transparent } \\
\text { information processing system. } \\
\text { Support new applications of very high } \\
\text { bandwidth communications demonstrating the } \\
\text { integration of research and education. } \\
\text { Increase emphasis on wireless } \\
\text { communications and wireless network access, and } \\
\text { on very broadband networks including optical } \\
\text { networks. }\end{array}$}} & HuCs & & \\
\hline & & & & & ETHR & & \\
\hline & & & & & \multicolumn{3}{|c|}{ Agency Ties } \\
\hline & & & & & DARPA & Par & tner \\
\hline & & & & & NSF & & \\
\hline & & & & & $\mathrm{DOE}$ & & ser \\
\hline & & & & & NASA & & ser \\
\hline & & & & & $\mathrm{NIH}$ & & \\
\hline & & & & & NSA & & \\
\hline & & & & & NIST & & \\
\hline & & & & & NOAA & & \\
\hline & & & & & EPA & & \\
\hline & & & & & ED & & \\
\hline & & & & & AHCPR & & \\
\hline & & & & & VA & & \\
\hline
\end{tabular}




\begin{tabular}{|c|c|c|}
\hline NSF & Applications & Budget Code: \\
\hline
\end{tabular}

Societal goals and the pursuit of fundamental knowledge in science and engineering identify the applications to be pursued. These applications fall into the following three categories:

High Performance Applications for Science and Engineering: These applications are intended to push the envelope of computational capabilities in order to enable new discoveries in science and engineering. Thus they require access to the highest performance computing systems available, interconnected by high speed networks. The Grand Challenge problems fall into this category.

High Confidence Applications for Dynamic Enterprises: These applications are intended to push the envelope of information processing in order to demonstrate and advance new technologies in the Information Age. Improvements in integration, privacy, security, and reliability of information flows within and across organizations are a consequence of pursuing these applications. Some of the National Challenge problems are examples of this type of application.

High Capability Applications for the Individual: These applications are focused on societal needs and are enabled by universal, easy to use access to information resources, powerful methods of presenting information for ease of understanding, and customization of 'information space' for personal use. National Challenges such as digital libraries and medical information servers are examples of this type of application.

These applications have one or both of the following attributes. They will drive and stress the enabling research areas outlined in the descriptions of Computing Systems, Human Centered Systems, and Networking, Communications, and the Convergence of Computing \&Communications given elsewhere in this document; they will lead to a paradigm shift in the application area involving a fundamentally different way of solving an important class of problems. The applications come from the physical and biological sciences, geosciences, social and behavioral sciences, and engineering.

\section{Milestone
Changes}

FY 1995 Actual Milestones

These are reported under Grand Challenges Applications Groups,Biological Sciences, Engineering, Geosciences, Computational Engineering, Geosciences, Computational $\quad$ Engineering, Geosciences, Computational and Economic Sciences, and National Challenges.
FY 1996 Estimated Milestones These are reported under Grand Challenges Applications Groups,Biological Sciences, Mathematics, Physical Sciences,Social,

Behavioral, and Economic Sciences, and National Challenges.

\section{FY}

FY 1997 Agency Request Milestones

Continue support of the Grand Challenges and National Challenges initiated in FY 1993, FY 1994, and FY 1995.

Continue to support the paradigm shift involving increased use of high performance computing to enhance or replace the experimental phase of the scientific method.

Continue the Digital Library projects, a joint research initiative with DARPA and NASA, and support related new work in very large databases and knowledge repositories.
Budget (\$ M)

\begin{tabular}{|l|l|}
\hline FY 95 Act & \\
\hline FY 96 Pres. & \\
\hline FY 96 Est. & \\
\hline FY 97 Rqst. & 55.21 \\
\hline
\end{tabular}

FY 97 Rqs

Program

Component

Areas

FY 96 FY 97

\begin{tabular}{|c|c|}
\hline $\mathrm{HECC}$ & 25.49 \\
\hline LSN & 8.20 \\
\hline HCS & \\
\hline HuCS & 9.66 \\
\hline ETHR & 11.86 \\
\hline
\end{tabular}

11.86

\section{Agency Ties}

\begin{tabular}{|c|c|}
\hline DARPA & Partner \\
\hline NSF & \\
\hline DOE & \\
\hline NASA & Partner \\
\hline NIH & \\
\hline NSA & \\
\hline NIST & Partner \\
\hline NOAA & Partner \\
\hline EPA & \\
\hline ED & \\
\hline AHCPR & \\
\hline VA & \\
\hline
\end{tabular}

December 20, 1996 


\begin{tabular}{|c|c|c|}
\hline NSF & Education and Training & Budget Code: \\
\hline
\end{tabular}

$\checkmark$ The program in education and training is focused on increasing the pool of people with the knowledge, skills and insights to lead research in the science and technology required to make high performance computing and information processing more easily utilized, and to apply those developments in the pursuit of fundamental knowledge in all disciplines of science and engineering. A secondary goal is to increase the percentage of the populace with an understanding of the power of and opportunities for high performance computing and information processing in the 21 st century. Example activities include:

MOSIS - An activity training students and providing research infrastructure for the design and manufacture of custom VLSI chips.

Undergraduate Education - An activity which provides funding for new course and curriculum development in high performance computing and communication and information processing.

Research Experiences for Undergraduates - Opportunities for undergraduates to perform research in high performance computing and communication an information processing.

Postdoctoral Research Associates - Postdoctoral training in computational science and engineering and experimental computer science.

Pilot Educational Networks - Develop networks to develop, implement, test, and evaluate applications of computer and communications to education.

Network Infrastructure for Education - A joint CISE-EHR activity that addresses issues of large-scale networking for education.

\begin{tabular}{|l|r|}
\hline \multicolumn{2}{|c|}{ Budget (\$ M) } \\
\hline FY 95 Act & 20.66 \\
\hline FY 96 Pres. & 20.24 \\
\hline FY 96 Est. & 16.62 \\
\hline FY 97 Rqst. & 9.75 \\
\hline
\end{tabular}

Program

Component

Areas

FY $9 6 \longdiv { \text { FY } 9 7 }$

HECC

Milestone

Changes

\section{FY 1995 Actual Milestones}

Monitored and evaluated existing projects and create new testbeds to demonstrate scalability,

cost/benefits, policy, effective applications,

educational impact, and other characteristics of

successful user-driven models of computer

networking in education.

Coordinated the results from existing NIE projects

and establish a base of knowledge for new applicants

to the NIE and programs.

Coordinated NIE activities with ED Goals 2000

planning grants and with NTIA infrastructure awards.

Initiated pilot studies for educational

applications of Digital Library awards.

\section{FY 1996 Estimated Milestones}

Evaluate and analyze ongoing educational networking projects to create a base of knowledge for successful user-driven model transfer and expansion.

Support large-scale models for educational applications of Digital Libraries.

Monitor and evaluate existing projects that demonstrate scalability, cost/benefits, policy, effective applications and other characteristics of successful user-driven models of computer networking in education.

Develop plans for technology transfer of networked-based educational materials.

\section{FY 1997 Agency Request Milestones}

Increase support for the incorporation of research findings into the undergraduate curriculum.

Increase support for the integration of research and education through supplements to existing research awards and through the initiation of new programs.

Initiate a new program in Learning

Technologies that will combine human centered information systems research with research in education to provide the basis for the next generation of technologies for education.

\begin{tabular}{|l|l|l|}
\hline LSN & 2.50 & 2.50 \\
\hline HCS & & \\
\hline HuCS & & \\
\hline ETHR & 14.12 & 7.25 \\
\hline
\end{tabular}

\section{Agency Ties}

\begin{tabular}{|c|c|}
\hline DARPA & Partner \\
\hline NSF & \\
\hline DOE & \\
\hline NASA & Partner \\
\hline NIH & \\
\hline NSA & \\
\hline NIST & Partner \\
\hline NOAA & Partiner \\
\hline EPA & \\
\hline ED & \\
\hline AHCPR & \\
\hline VA & \\
\hline
\end{tabular}

December 20, 1996 


\begin{tabular}{|c|c|c|}
\hline NSF & Research Centers & Budget Code: \\
\hline
\end{tabular}

The HPCC program provides support for four Science and Technology Centers. These centers share several important characteristics: a unifying, cross-disciplinary intellectual focus; an emphasis on knowledge-transfer and linkages with private sector organizations; and significant education and outreach components. The centers and their focus are:

Center for Research in Parallel Computation, CRPC, at Rice University -- making parallel computers as easy to use as conventional computers;

Center for Computer Graphics and Scientific Visualization, CG \& SV, at the University of Utah -- building and displaying models that are visually and measurably indistinguishable from real world entities;

Center for Discrete Mathematics and Theoretical Computer Science, DIMACS, at Rutgers University -- applying discrete mathematics and theoretical computer science to the solution of fundamental problems in science and engineering;

Center for Cognitive Science at the University of Pennsylvania -- human cognition, perception, natural language processing, and the application of parallel computation.

Milestone

Changes

$$
\text { FY } 1995 \text { Actual Milestones }
$$

Released a set of software tools for the support of experimental analysis in discrete mathematics and theoretical computer science.

Released a portable prototype library for sparse direct matrix computation that can be optimized for different computing systems.

Developed an object-oriented multi-disciplinary analysis and design tool set to solve optimization problems in industrial design.

Demonstrated with biological modeling, a fluent, physically based modeling language that extends geometric modeling languages and operators.

Released a second version of High Performance Fortran that provides support for adaptive, dynamic computations.

Began a special year on Mathematical Support for Molecular Biology at DIMACS focused on the application of discrete mathematics and theoretical computer science on problems in DNA sequencing and in protein structure.

\section{FY 1996 Estimated Milestones}

Build models that are visually and measurably indistinguishable from real-world entities.

Provide universal Fortran-based language for which vendors can provide efficient compilers and which will assure program portability.

Identify and exploit applications areas where discrete mathematics and theoretical computer science developments can lead to more accurate and efficient computations.

Begin a special year on Logic and Algorithms at DIMACS focused on the relationship between mathematics and computational algorithms.

\section{FY 1997 Agency Request Milestones}

\section{This activity is now incorporated into the}

activities described under Computing Systems an Human-Centered Systems.
Budget (\$ M)

\begin{tabular}{|l|l|}
\hline FY 95 Act & 10.68 \\
\hline FY 96 Pres. & 11.08 \\
\hline FY 96 Est. & 11.38 \\
\hline FY 97 Rqst. & \\
\hline
\end{tabular}

FY 97 Rqst.

Program

Component

Areas

FY 96 FY 97

L

LS

HCS

HuCS

ETHR

4.40

Agency Ties

\begin{tabular}{|c|c|}
\hline DARPA & \\
\hline NSF & \\
\hline DOE & \\
\hline NASA & \\
\hline NIH & \\
\hline NSA & \\
\hline NIST & \\
\hline NOAA & \\
\hline EPA & \\
\hline ED & \\
\hline AHCPR & \\
\hline VA & \\
\hline
\end{tabular}

December 20, 1996 
The Research Infrastructure activity aids in the establishment,enhancement, and operation of major experimental facilities and in the acquisition of o. equipment such as workstations in order to support research activities in the areas of computer and information science, computer engineering, or computational science. In general, support is provided for equipment, maintenance, technical support staff, and other appropriate costs. The groups supported range from single researchers requiring workstations to cross departmental or cross institutional groups requiring access to special purpose instrumentation.

The largest part of the activity is the Institutional Infrastructure Program. The program activity supports both the acquisition of Scalable Parallel computers for research in parallel computing and computational science and engineering and the acquisition of facilities for experimental research.

The Research Instrumentation program, a smaller activity, supports experimental research with awards for workstations for experimental facilities and also for smaller Scalable Parallel computers.

\section{Milestone \\ Changes}

\section{FY 1995 Actual Milestones}

Supported infrastructure for research on the

input/output problems with scalable parallel system

Developed infrastructure to support networked applications in education and advanced

manufacturing.

Provided infrastructure support for gigabit network research.

Provided infrastructure support for virtual

manufacturing.

Provided infrastructure support for multi-media systems research.

Continued support of identified infrastructure needs for basic experimental research.

Participated in the Network Infrastructure for Education (NIE) program supporting infrastructure for education applications of the NII.

\section{FY 1996 Estimated Milestones}

Conduct formal evaluation of existing programs to adjust to the infrastructure needs of experimental research in computing disciplines supporting HPCC.

Develop infrastructure support for basic research necessary for educational uses of the NII.

Continue infrastructure support for virtual manufacturing, and other applications of information infrastructure technologies

Increase participation in the NIE program.

\section{FY 1997 Agency Request Milestones}

This activity is now incorporated into the activities described under Computing Systems, Human-Centered Systems, Networking,

Communications and the Convergence of Computing and Communications, and Applications.
Budget (\$ M)

\begin{tabular}{|c|c|}
\hline FY 95 Act & 19.23 \\
\hline FY 96 Pres. & 21.59 \\
\hline FY 96 Est. & 18.20 \\
\hline FY 97 Rqst. & \\
\hline
\end{tabular}

Program

Component

Areas

\begin{tabular}{|l|l|} 
FY 96 & FY 97 \\
\hline
\end{tabular}

HECC 8.64

\begin{tabular}{|l|l|l|}
\hline LSN & 2.48 & \\
\hline HCS & & \\
\hline HuCS & 4.78 & \\
\hline ETHR & 2.30 & \\
\hline
\end{tabular}

Agency Ties

\begin{tabular}{|c|c|}
\hline DARPA & \\
\hline NSF & \\
\hline DOE & \\
\hline NASA & \\
\hline NIH & \\
\hline NSA & \\
\hline NIST & \\
\hline NOAA & \\
\hline EPA & \\
\hline ED & \\
\hline AHCPR & \\
\hline VA & \\
\hline
\end{tabular}

December 20, 1996 


\begin{tabular}{|c|c|c|}
\hline NSF & Grand Challenge Applications Groups & Budget Code: \\
\hline
\end{tabular}

This activity supports multidisciplinary groups of scientists, engineers, mathematicians, and computer scientists to apply emerging high performance computing and communications systems to advance the solution of fundamental problems in science and engineering. These activities will generate

significant new research in mathematics, computer science, engineering, and other scientific disciplines. The groups are listed below.

High Performance Computing for Learning--Massachusetts Institute of Technology

High Performance Computing for Land Cover Dynamics--University of Maryland

Black Hole Binaries: Coalescence and Gravitational Radiation--University of Texas

High Performance Imaging in Biological Research--Carnegie Mellon University

Earthquake Ground Motion Modeling in Large Basins--Carnegie Mellon University

Computational Biomolecular Design--University of Houston

Adaptive Coordination of Predictive models with Experimental Observations--Stanford University

The Formation of Galaxies and Large-Scale Structure--Princeton University

High Performance Computational Methods for Coupled Field Problems and GAFD Turbulence--University of Colorado at Boulder

Radio Synthesis Imaging: An HPCC Application--University of Illinois at Champaign-Urbana

High Capacity Atomic-Level Simulations for Design of Materials Modeling--Carnegie Mellon University

A Distributed Computational System for Large Scale Environmental Modeling--California Institute of Technology

Parallel I/O Methodologies for I/O-Intensive Grand Challenge Applications--California Institute of Technology

Understanding Human Joint Mechanics Through Advanced Computational Models--Rensselaer Polytechnic Institute

Massively Parallel Simulation of Large Scale, High Resolution Ecosystem Models Determination--University of Illinois

Advanced Computational Approaches to Biomolecular Modeling and Structure--University of Arizona

\section{Milestone}

Changes
FY 1995 Actual Milestones

Demonstrated the utility of direct sensitivity analysis in air quality models such as those for the Los Angeles basin or the Northeast Corridor.

Demonstrated the integrated AIM microscope

develop capability to automatically detect events of interest.

Demonstrated on high performance platforms such as the CM-5 a simulation of a watershed using a discrete event dynamic model coupled to a geographic information system data base with visualization on a SGI-based system.

Brought the real-time transfer of data from the data archive to full, routine operational status.

Incorporated Fast Multipole type methods into molecular dynamics simulations of large biomolecular systems.

Made five new Grand Challenge awards. system for monitoring biomedical experiments and BIMA telescope system in California to the NCSA

\section{FY 1996 Estimated Milestones}

Extend face recognition system to larger data sets including recognition in groups.

Release of Scientist's Visual Workbench to astronomers, with development versions of

interactive, distributed visualization and remote rendering of very large images.

Demonstrate effectiveness of Archimedes, a special purpose compiler for unstructured mesh computations, through simulation of the seismic response of Los Angeles Basin.

Enhance the CM-5 watershed simulation model with hydrological/ecological relationships to

demonstrate increased realism within reasonable run times.

Begin work with industry to produce an affordable, stand- alone successor to AIM, with specialized high performance computing hardware built in.
FY 1997 Agency Request Milestones

This activity is now incorporated into the activity described under Applications.

\section{Budget (\$ M)}

\begin{tabular}{|l|r|}
\hline FY 95 Act & 12.83 \\
\hline FY 96 Pres. & 7.25 \\
\hline FY 96 Est. & 7.32 \\
\hline FY 97 Rqst. & \\
\hline
\end{tabular}

Program

Component Areas FY 96 FY 97

HECC 7.32

LSN

HCS

Agency Ties

\begin{tabular}{|c|c|}
\hline DARPA & Partner \\
\hline NSF & \\
\hline DOE & \\
\hline NASA & \\
\hline NIH & \\
\hline NSA & \\
\hline NIST & \\
\hline NOAA & \\
\hline EPA & User \\
\hline ED & \\
\hline AHCPR & \\
\hline VA & \\
\hline
\end{tabular}

January 22, 1997 


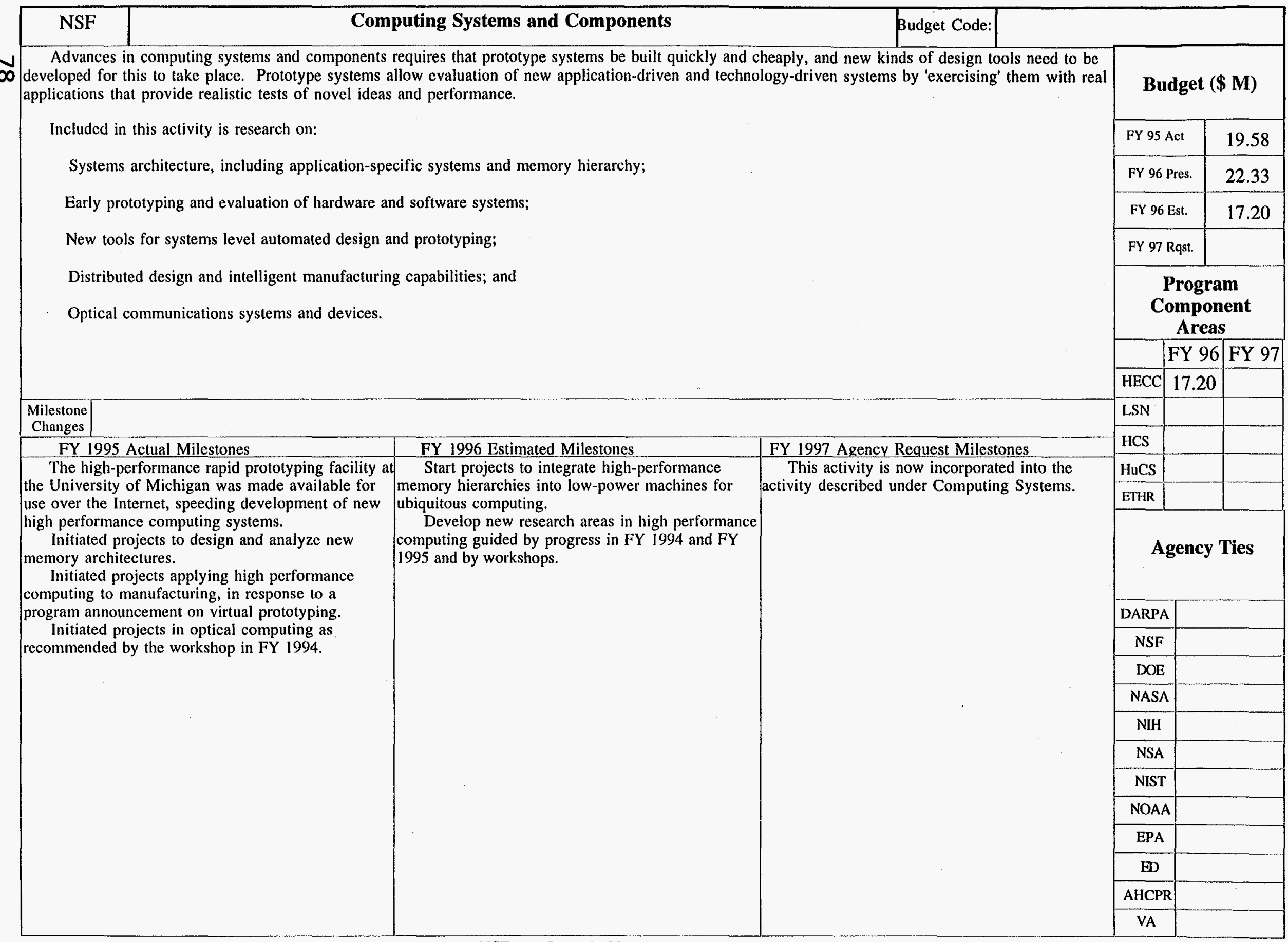




\begin{tabular}{|c|c|c|c|}
\hline NSF & Software Systems and Algorithms & Budget Code: & $\mid$ \\
\hline Research emphasizes the development of scalable parallel algorithms and software technologies, and the development of problem solving environments.
\end{tabular}

Areas of research include:

Parallel languages and optimizing compilers;

Parallel operating systems;

Performance evaluation and prediction;

High performance systems for numeric and symbolic computations;

Parallel algorithms and data structures;

Algorithms for biological applications;

Problem solving environments;

Software engineering;

Computer graphics;

Computational geometry; and

Real-time systems.

In addition, a significant fraction of the activity is devoted to fundamental research, with potential impact on high performance computing, in areas such as the theory of computing,software engineering, and theoretical aspects of computer systems and operating systems.

The activity also includes a postdoctoral program for interdisciplinary computational scientists.

\section{Milestone}

Changes

\section{FY 1995 Actual Milestones}

Developed compilers for high performance

Fortran for several parallel machines.

Developed scalable multiprocessing operating systems.

Developed efficient probabilistic algorithms

based on information-based complexity for importan scientific problems.

Demonstrated software engineering techniques ford

developing and analyzing designs and software with

real-time or other critical dependability constraints.

Demonstrated software to support transparent

computations on a heterogeneous collection of

computing systems that are geographically

distributed.

Developed program of Challenges in Computer Science to support research on technologies that wil

enhance the usability of high performance computing

systems.

ખে

\section{FY 1996 Estimated Milestones}

Increase the support for compiler and operating systems research focused on heterogeneous,

geographically distributed collections of computing systems.

Introduce a sabbatical program to support

scientists and engineers to become more proficient

in applying paraliel computing technology to their

Initiate a program to bring promising computer science research to a point of maturity that will permit evaluation by the user community.

Identify and exploit applications areas where

discrete mathematics and theoretical computer

science developments can lead to more accurate

and efficient computations.

Extend ongoing research in parallel and

distributed computing to address problems for

universal services including heterogeneity,

interoperability, and real-time operating systems.
FY 1997 Agency Request Milestones

This activity is now incorporated into the

activities described under Computing Systems and

Networking, Communications and the

Convergence and Computing and

Communications.
Budget (\$ M)

\begin{tabular}{|c|c|}
\hline FY 95 Act & 29.82 \\
\hline FY 96 Pres. & 29.01 \\
\hline FY 96 Est. & 26.66 \\
\hline FY 97 Rqst. & \\
\hline
\end{tabular}

Program

Component

Areas

FY 96 FY 97

\begin{tabular}{|l|l|l|}
\hline HECC & 26.66 & \\
\hline LSN & & \\
\hline HCS & & \\
\hline HuCS & & \\
\hline ETHR & & \\
\hline
\end{tabular}

ETHR

Agency Ties

\begin{tabular}{|c|c|}
\hline DARPA & \\
\hline NSF & \\
\hline DOE & \\
\hline NASA & \\
\hline NIH & \\
\hline NSA & \\
\hline NIST & \\
\hline NOAA & \\
\hline EPA & \\
\hline ED & \\
\hline AHCPR & \\
\hline VA & \\
\hline
\end{tabular}

December 20, 1996 
The focus of this program activity is basic research on technology for the transfer of information from one point in space to another (transmission and networking) and/or one point in time to another (information storage and retrieval) including multiple access techniques to enable effective collaborative access to information resources. Research issues include: the efficient utilization of spectral bandwidth for wireless and wired systems; new architectures and multiple access techniques for lightwave systems; optical technologies; reliable and secure transmission or storage of information in local and in multiple access environments; and fundamental aspects of the characterization, control, and management,of information networks.

Areas of currently funded research include:

Gigabit testbed research;

Design and analysis of gigabit switching systems;

Protocols and software structures for network management;

Resource discovery among collaborative information spaces in large, decentralized environments;

Network information theory;

Multi-sender and multi-receiver network security;

Modulation, detection, and coding of reliable information storage and retrieval;

All optical networks; and

Optical technologies for computing and communications.

Budget (\$ M)

\begin{tabular}{|c|c|}
\hline FY 95 Act & 10.52 \\
\hline FY 96 Pres. & 14.80 \\
\hline FY 96 Est. & 14.44 \\
\hline FY 97 Rqst. & \\
\hline
\end{tabular}

Program

Component

Areas

FY $9 6 \longdiv { \text { FY } 9 7 }$

Milestone The name of this activity has been changed from Very High Speed Networks and Communications in the previous Implementation Plan to reflect the broader Changes nature of the program.

\section{FY 1995 Actual Milestones}

Reported on the accomplishments of the gigabit testbed project,including lessons learned and the

strengths and weaknesses of technologies deployed in the testbeds.

Sponsored a conference in November to discuss testbed results.

Held workshops on Wireless Access, Open

Bearer Service, and Network Storage.
FY 1996 Estimated Milestones

Build on new gigabit research infrastructure by funding research aimed at more effective utilization access to, distribution of, and effective interactions Computing and Communications. with these resources.

Hold competitive program solicitation and make awards for wireless access testbeds.

Continue collaboration with ARPA in $10 \mathrm{~Gb} / \mathrm{s}$ testbed initiated under the Technology Reinvestment Program.

Continue basic research in coding and coded modulation relevant to challenges in wireless acces such as mobility management and channels of widely and rapidly fluctuating capacity.

Examine convergence of computing, entertainment, and telecommunications from the standpoint of the Internet experience and the Open Bearer Service.

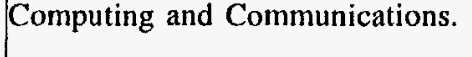
Computing and Communications.

(

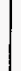

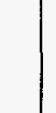

\section{FY 1997 Agency Request Milestones}

This activity is now incorporated into the ergence of

Held workshops on Wireless Access, Open
Bearer Service, and Network Storage.




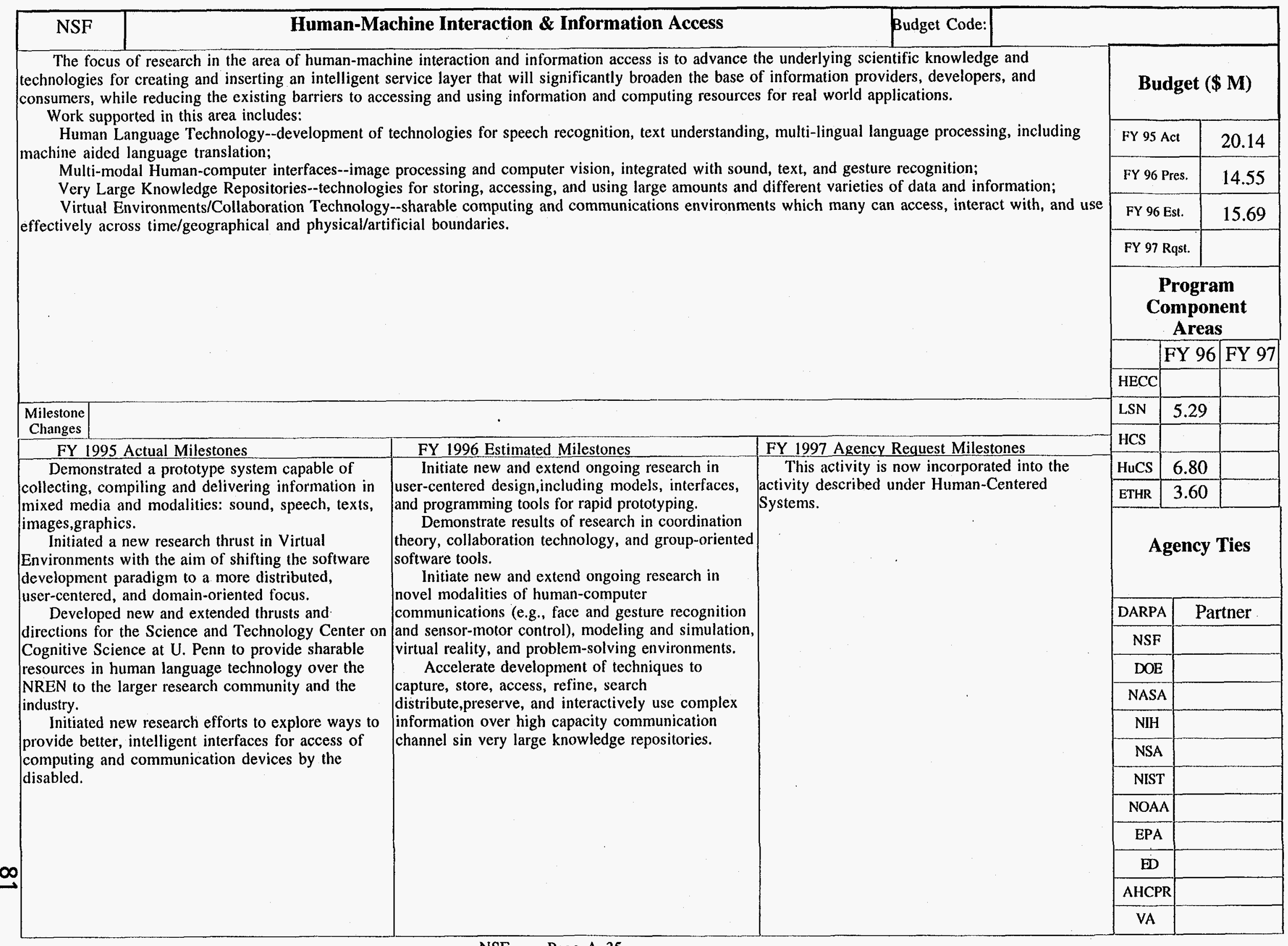


Many areas of both basic and applied biology require the use of computational tools to resolve significant questions. HPCC has had a dramatic impact on many of these areas. Three examples are mentioned below. Each one must analyze experimental results, construct detailed theory and then interrelate the two. They must also develop database systems to handle heterogeneous databases and to develop networking technologies to link various databases. This program activity supports the development of necessary computational tools: software; optimization techniques; and large volume data accessing, storage and managing techniques. In addition, the activity supports modeling of biological phenomena on new computer architectures, which will allow new questions to be posed and answered in these areas. In many cases projects are supported that involve multidisciplinary groups of investigators.

Structural Biology: The challenge is to determine macromolecular structure, and to relate structure to function. Determining structure of a molecule directly from experimental data or inferring it through comparison with known structures are computationally intensive problems that will not be solved without HPCC systems. The impact of solving these problems will be felt in both basic and applied biology, e.g., rational drug design, as well as biomaterials.

Neuroscience: The challenge is to understand how networks of neurons work, and ultimately how the entire nervous system operates, from sensory systems to learning and memory. New HPCC architectures allow for explorations of these questions. The impact of further understanding of experimental and theoretical systems will be felt in the areas of health care (neuropharmacology, psychotherapy), artificial intelligence, and robotics.

Ecology: The challenge is to understand the relationship of individuals that interact at various scales, both spatial -- from microorganisms to higher organisms, from populations to entire ecosystems, ultimately to global scale -. and temporal .- from nanoseconds to geological time. The complexity of the data and of the relationships requires computational solutions. Impact of advances in this area will be felt on issues of global change, biodiversity, and the environment.

Milestone Several Milestones listed below contribute to and benefit from other NSF themes such as Ubiquitous Computing and Communication and Human-Machine Changes Interaction \& Information Access.

\section{FY 1995 Actual Milestones \\ Developed collaborative internet research} environments (e.g., Khoros).

Established the Center for Ecological Analysis and Synthesis, prototyping the linking of heterogeneous databases.

Continued development and distribution of computational tools for molecular dynamics,neural simulators, and ecological simulation studies, with aims of increasing size and realism of simulations.

$\mid$

\section{FY 1996 Estimated Milestones}

Develop prototype information workplace of the

21 st century including tools for use and accessibility

of heterogeneous (multimedia) distributed

databases.

Develop search tools for databases of images.

Develop postdoctoral and training programs in the use of high performance computing and information technology.

Begin partnerships with other agencies regarding information infrastructure support and maintenance, e.g., the Network of Networks.

Continue development of the Center for Ecological Analysis and Synthesis, and of models and tools to make computer simulations of biological phenomena more realistic over temporal and spatial scales.

\section{FY 1997 Agency Request Milestones}

This activity is now incorporated into the ctivity described under Applications.
Budget (\$ M)

\begin{tabular}{|c|c|}
\hline FY 95 Act & 9.89 \\
\hline FY 96 Pres. & 11.43 \\
\hline FY 96 Est. & 12.42 \\
\hline FY 97 Rqst. & \\
\hline
\end{tabular}

Program

Component

Areas

FY $9 6 \longdiv { \text { FY } 9 7 }$

HECC

\section{\begin{tabular}{l} 
LSN \\
\hline HCS
\end{tabular}}

HCS

\begin{tabular}{l} 
HuCS \\
\hline ETHR
\end{tabular}

Agency Ties

\begin{tabular}{|c|c|}
\hline DARPA & \\
\hline NSF & \\
\hline DOE & \\
\hline NASA & \\
\hline NIH & \\
\hline NSA & \\
\hline NIST & \\
\hline NOAA & \\
\hline EPA & \\
\hline ED & \\
\hline AHCPR & \\
\hline VA & \\
\hline
\end{tabular}

December 20, 1996 


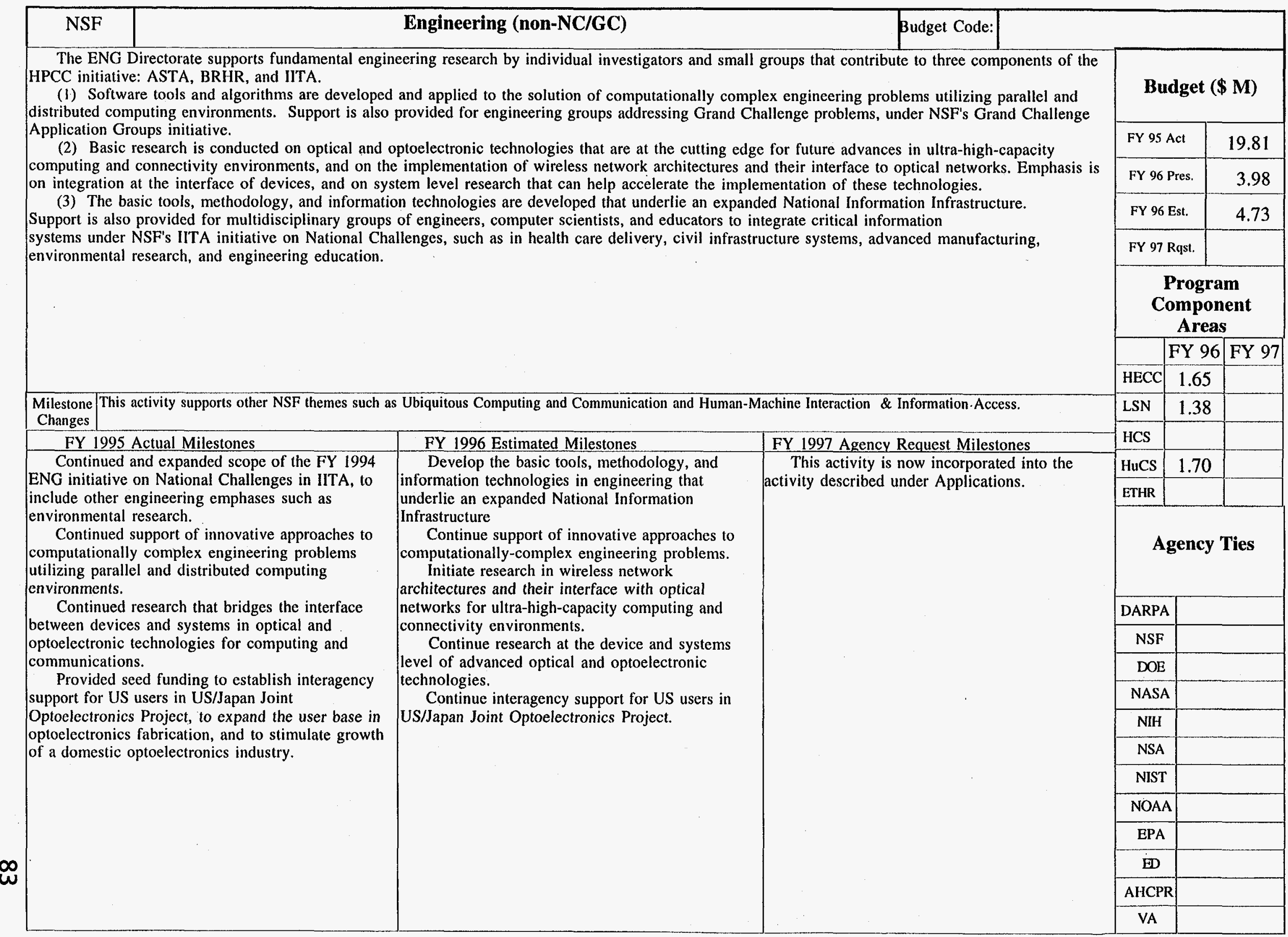




\begin{tabular}{|c|c|c|}
\hline NSF & Geosciences (non-NC/GC) & Budget Code: \\
\hline
\end{tabular}

$\infty$ Geosciences and the Office of Polar Programs HPCC activities build on a substantial infrastructure within the directorate. Incremental funds are used to enhance present activities and to synergistically integrate existing efforts into a geosciences program and the Global Change Research Program that is responsive and effective in using emerging technologies. The acquisition and testing (using Geoscience codes) of parallel computers of various architectures is being carried out to determine which technology offers the most promising capabilities. This initial work will guide the acquisition of a massively parallel machine in the FY 95-97 time frame.

Software that effectively uses parallel computers for geoscience problems is being developed and enhanced, including parallel algorithms to be used in models such as the Community Climate, global atmospheric chemistry, and eddy-resolving global ocean circulation models.

Extended activities are carried out to provide access to unique scientific and informational resources by connecting the Antarctic science facilities to the Internet for high speed data transmission (especially during the southern hemisphere winter) of global data,images from telescopes, and general interactive tele-science from remotely located experimental centers.

The education and training of geoscientists in high performance computing and communication is being addressed through a broad spectrum of courses ranging from program son a parallel computer to effective visualization techniques. These courses are offered at NCAR and the other NSF supercomputer centers. Graduate assistantships and postdoctoral positions that focus on HPCC activities are established at NCAR.

Technologies are being developed for Internet distribution of real-time geophysical data that have multiple sources at separate locations. Geoscience data necessary for monitoring global change is widely distributed with total databases anticipated to grow to 10 's of petabytes. The development of services, standards, tools, and user interfaces for storing, finding, transmitting,manipulating, displaying, comparing, and analyzing three dimensional historical and near real-time geophysical data is a major activity. It is the aim of this effort to allow a university user transparent access to petabytes of geosciences data located at several centers from which data of interest can be selected.

\section{Milestone}

Changes

\section{FY 1995 Actual Milestones}

Demonstrated Earth Simulation models simultaneously running on MPP, clustered and shared memory computers and connected by

'flux-coupler' software and high speed networking technology.

Upgraded Climate Simulation Laboratory 5 processor CRAY Y-MP/T3D machine to 8 processor CRAY Y-MP.

Completed partial implementation of internet data distribution.

Together with NASA and NOAA demonstrated the $256 \mathrm{Kbps}$ data transmission between the U.S. and South Pole Station.
FY 1996 Estimated Milestones

Replace shared memory computer at NCAR and upgrade MPP machines.

Complete final development phase of Earth

Simulation models running on different computers.

Establish preliminary Volcano Network and fully implement geophysical data distribution.

Establish an HPCC graduate and postdoctoral program for geosciences and revise exiting postdoctoral program to add HPCC thrust. Six to eight people will be supported.

Enhance Mass Store software to effectively work with high performance MPP computers,

Bring to operation 1.5Mbps link at Palmer Station, Antarctica using ACTS satellite.

\section{FY 1997 Agency Request Milestones}

This activity is now incorporated into the activity described under Applications.
Budget (\$ M)

\begin{tabular}{|c|c|}
\hline FY 95 Act & 4.28 \\
\hline FY 96 Pres. & 3.59 \\
\hline FY 96 Est. & 4.66 \\
\hline FY 97 Rqst. & \\
\hline
\end{tabular}

Program

Component

Areas

FY 96 FY 97

HECC 3.19

LSN

1.47

HCS

HuCS

ETHR

Agency Ties

\begin{tabular}{|c|c|}
\hline DARPA & \\
\hline NSF & \\
\hline DOE & \\
\hline NASA & Partner \\
\hline NIH & \\
\hline NSA & \\
\hline NIST & \\
\hline NOAA & Partner \\
\hline EPA & \\
\hline ED & \\
\hline AHCPR & \\
\hline VA & \\
\hline
\end{tabular}

December 20, 1996 


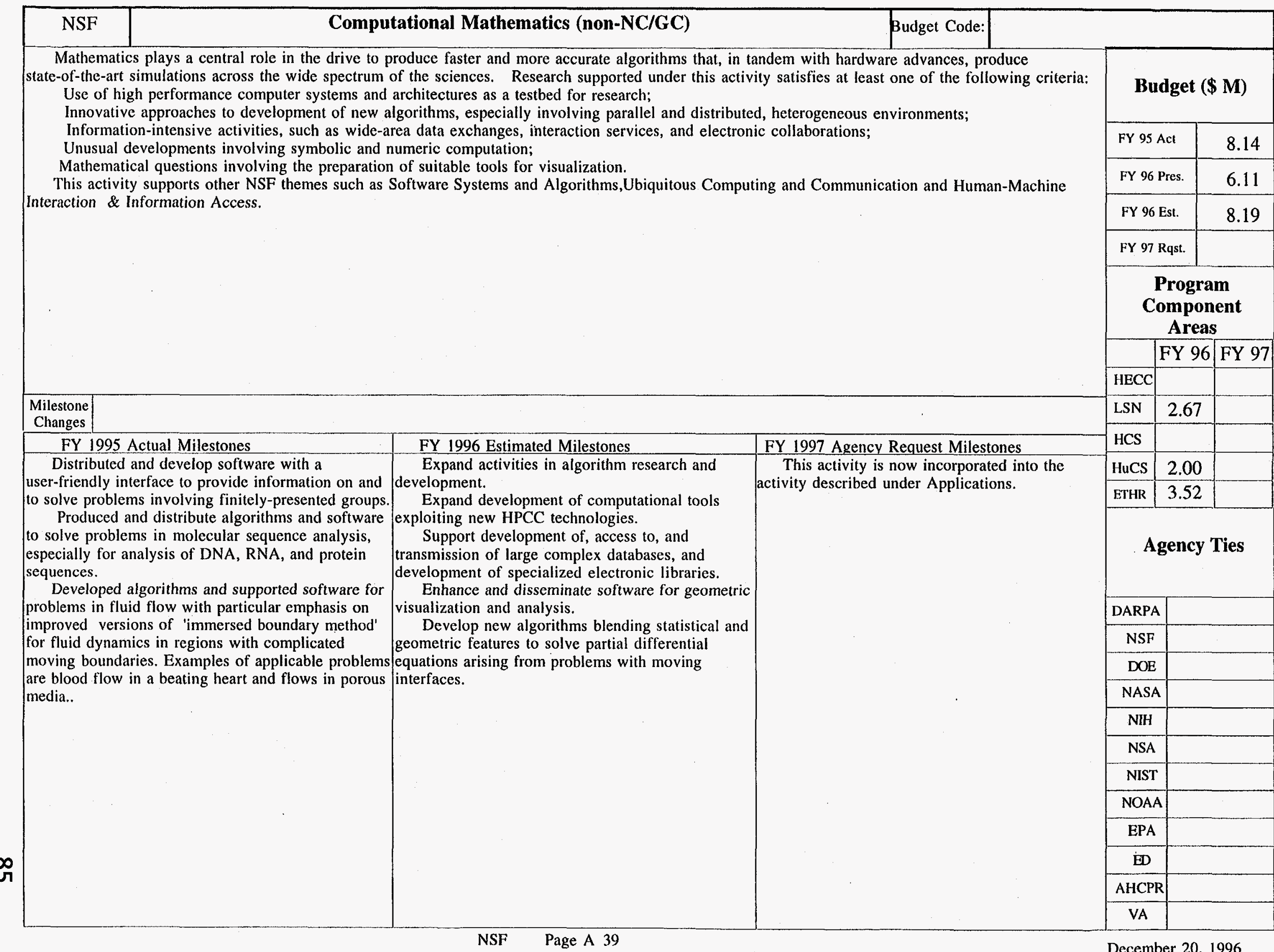


$\infty$ This activity provides support for research on fundamental problems in Astronomy, Chemistry, Material Science, and Physics using state of the art vector supercomputers or emerging massively parallel systems. The emphasis is on developing models and solution techniques that provide for qualitative and quantitative improvements in the simulations. From these more accurate simulations the researchers are able to gain new insights into the nature of the physical phenomena being simulated. In addition, this activity broadens the base of users of advanced computing systems by exposing graduate students and postdoctoral researchers to the benefits of computational science as an intrinsic part of the scientific method.

This activity supports other NSF themes such as Software Systems and Algorithms,Ubiquitous Computing and Communication, and Human-Machine Interaction \& Information Access.

\section{Milestone
Changes}

\section{Changes}

Demonstrated the efficacy of parallelized $\mathrm{CMM} / \mathrm{MD}$ codes by using them to predict glass transition temperatures of polymer blends and copolymers.

Extended the Newton-Euler Inverse Mass Operator method to fast dynamics on periodic systems with up to one million atoms.

A new project to enable scientists in physics and other disciplines to acquire and distribute information easily and rapidly in the form of electronic preprints was initiated.

The interdisciplinary CARM program that supports high performance computing research in the material sciences was expanded to include a wider variety of materials, algorithms and computing platforms.

Linkages between NSF and NIST were explored in the area of computational materials.

\section{FY 1996 Estimated Milestones}

Working parallelized versions of GAUSSIAN

FY 1997 Agency Request Milestones CHARM, or other molecular dynamics codes will belactivity described under Applications.

available for public use at all of the NSF

Supercomputer Centers.

Through the Supercomputer Centers and the parallelized versions of codes supported for public

use, strengthen the ties between academic

searchers and industrial affiliates.

As appropriate, establish interactions between NSF and NIST in applications of HPCC to computational modeling of materials.

Expand the CARM program to include computation for materials engineering problems.

Develop rapid, open, and widespread access to electronic preprints over a broad spectrum of physics, chemistry, astronomy, and materials science.

\section{Budget (\$ M)}

FY 95 Act

FY 96 Pres

FY 96 Est.

FY 97 Rqs

Program

Component

Areas

FY 96 FY 97

HECC

6.41

HCS

HuCs

ETHR

3.09

Agency Ties

\begin{tabular}{|c|c|}
\hline DARPA & \\
\hline NSF & \\
\hline DOE & \\
\hline NASA & \\
\hline NIH & \\
\hline NSA & \\
\hline NIST & partner \\
\hline NOAA & \\
\hline EPA & \\
\hline ED & \\
\hline AHCPR & \\
\hline VA & \\
\hline
\end{tabular}

December 20, 1996 


\begin{tabular}{|c|c|c|}
\hline NSF & Social, Behavioral \& Economic Sciences (non-NC/GC) & Budget Code: \\
\hline
\end{tabular}

The Division of Social, Behavioral and Economic Research(SBER) was created at the beginning of 1993, by amalgamation of many existing programs. SBER is currently engaged in the development of a general plan for computing in the social and behavioral sciences, and it is examining how best to contribute to the HPCC component, IITA. There are five program clusters in SBER, and each one has held a workshop on aspects of high performance computing that relate to its disciplines:

Cognitive, Psychological, and Language Sciences cluster: 'Cognitive Science' workshop;

Anthropological and Geographic Sciences cluster: 'Computational Geography' workshop;

Economics, Decision and Management Sciences cluster: 'Computational Economics' workshop;

Social and Political Sciences cluster: Artificial Social Intelligence' workshop; and

Science, Technology and Society cluster: 'Electronic Networks' workshop.

SBER will be heavily involved in the IITA component, because its goals are close to the social and behavioral sciences.

\section{Milestone}

Changes

FY 1995 Actual Milestones

Established distributed processing geographic information systems for the National Information Infrastructure.

Adapted genetic algorithms, neural networks, and symbolic processors to a wide range of problems in
the social sciences.

Demonstrated new methods for providing wide access to previously unavailable government statistics, while preserving data integrity and preventing misuse.
FY 1996 Estimated Milestones

Launch new competition for multi-disciplinary research groups to conduct research in advanced computing for the social, behavioral, and economic

FY 1997 Agency Request Milestones

This activity is now incorporated into the activity described under Applications.

Support work on software and mathematical tools for the National Information Infrastructure.

Develop Cognitive Science activity in a partnership of the programs in Linguistics and in Human Cognition and Perception.

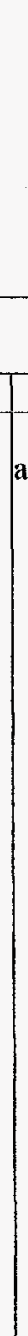




\begin{tabular}{|c|c|c|}
\hline NSF & National Challenges & Budget Code: \\
\hline
\end{tabular}

$\infty$ National Challenges are information intensive applications that have broad and direct impact on the Nation's well-being and economic competitiveness.

$\infty$ The IITA component supports the use and integration of component technologies developed in other parts of HPCC to seek solutions to such applications.

The research needed to accelerate these solutions will span a spectrum of activities from concept demonstrations and experimental testbeds to the actual delivery of application systems in specific domains. Areas identified for focus include:

Digital Libraries;

Advanced Manufacturing;

Education, Training, and Lifelong Learning;

Health Care;

Civil Infrastructure Systems;

Physical, Biological, and Geological Network Centers;

Government Information Delivery;

Environmental Monitoring;

Electronic Commerce;

Crisis Management;

Energy Management.

Initially, the NSF program will focus on the first seven activities.

\section{Milestone \\ Changes}

FY 1995 Actual Milestones

Developed a supplemental program for additional institutions to participate in the Digital Libraries initiative.

Demonstrated preliminary models and data developed by the research teams under the cross-agency Digital Libraries initiative.

Implemented a cross-disciplinary research program on Virtual Prototyping for Advanced Manufacturing.

Developed and implemented a program on

Reliable Computer Controlled Systems to accelerate progress in providing safe and reliable computing and communication devices and systems for National Challenge application areas.

Developed laboratories as part of the new institutional infrastructure needed for investigating effective human utilization of massive information stores.

Conducted a new round of competition for National Challenges.

\section{FY 1996 Estimated Milestones}

Demonstrate preliminary results of research and the experimental testbeds developed under the Digital Libraries initiative.

Demonstrate results of research from National

Challenges through workshops and symposia.

Initiate new research to accelerate development of enabling technologies (e.g., wireless, lightwave)

for the NII to help provide universal access to

diverse bitways, including high speed networks. Expand programs in National Challenges.
Budget (\$ M)

\begin{tabular}{|c|c|}
\hline FY 95 Act & 7.28 \\
\hline FY 96 Pres. & 17.33 \\
\hline FY 96 Est. & 8.55 \\
\hline FY 97 Rqst. & \\
\hline
\end{tabular}

Program

Component

Areas

FY 96 FY 97

LSN

8.55

This activity is now incorporated into the activity described under Applications.

\begin{tabular}{|l|l|l|}
\hline HCS & & \\
\hline HuCS & & \\
\hline ETHR & & \\
\hline
\end{tabular}

Agency Ties

\begin{tabular}{|c|c|}
\hline DARPA & Partner \\
\hline NSF & \\
\hline DOE & \\
\hline NASA & Partner \\
\hline NIH & \\
\hline NSA & \\
\hline NIST & \\
\hline NOAA & \\
\hline EPA & \\
\hline ED & \\
\hline AHCPR & \\
\hline VA & \\
\hline
\end{tabular}

December 20, 1996 


\section{Department of Energy}

DOE faces unprecedented challenges challenges as it approaches the 21st century. DOE is committed to:reducing the global nuclear danger through its national security and nonproliferation activities; replacing underground nuclear testing with science; understanding and dealing with risks associated with environmental problems resulting from nuclear weapons production during the Cold War; promoting clean and efficient supply of energy; ensuring continuing US world leadership in science and technology research; and maintaining U.S. global competitiveness through leadership in environmentally-conscious materials, technologies, and industrial processes.

The DOE High Performance Computing Program is focused on providing DOE with tools to address these challenges. It is a forefront, diverse applied mathematical sciences, high performance computing, communications and information infrastructure program which spans the spectrum of activities from strategic fundamental research to technology development and demonstration. The diverse activities supported by this program are integrated to support two major strategic thrusts: National Collaboratories (NC) and Advanced Computational Testing and Simulation (ACTS).

The thrust in National Collaboratories is developing a set of tools and capabilities which will permit scientists and engineers working at different DOE and other facilities to collaborate on solving problems as easily as if they were in the same building.

The thrust in Advanced Computational Testing and Simulation is developing an integrated set of algorithms, software tools and infrastructure which will enable computer simulation to be used in place of experiments when real experiments are too dangerous, expensive, inaccessible, or politically infeasible. These two strategic thrusts support the underlying mathematical concepts and information technology needs of all Department of Energy (DOE) mission areas (e.g., Defense, Energy Efficiency, Environmental and Fossil programs, etc.) and the efforts in these areas are closely coordinated with related activities supported by Defense Programs.

The DOE program also supports and responds to the Energy Policy Act (EPACT) and to the High Performance Computing Act of 1991 (also known as the Gore Bill) and provides supercomputer access and advanced communication capabilities (through the ESnet computer network) to scientific researchers. Finally, the this program also serves as an advocate within the Department to formulate and coordinate the Department's National Information Infrastructure (NII) initiative, especially to promote economically beneficial energy-related 'National Challenges' applications such as energy demand and supply management and to develop the underlying technologies to enable these applications. 


\section{Department of Energy}

\begin{tabular}{|c|c|c|c|c|c|c|c|c|c|c|c|}
\hline \multirow[b]{2}{*}{ Program Activity } & \multirow[b]{2}{*}{ Budget Account Code } & \multirow{2}{*}{$\begin{array}{c}\text { Partner/User } \\
\text { Agencies }\end{array}$} & \multicolumn{4}{|c|}{ Budget $(\mathrm{BA}, \$ \mathrm{M})$} & \multicolumn{5}{|c|}{ HPCC PCAs by 1997 Pres. Request } \\
\hline & & & $\begin{array}{l}\text { FY 95 } \\
\text { Actual } \\
\end{array}$ & $\begin{array}{c}\text { FY } 96 \\
\text { Pres. }\end{array}$ & $\begin{array}{c}\text { FY } 96 \\
\text { Est. }\end{array}$ & $\begin{array}{c}\text { FY } 97 \\
\text { Rqst. }\end{array}$ & HECC & LSN & HCS & HuCS & ETHR \\
\hline $\begin{array}{l}\text { Advanced Computational Testing and Simulation } \\
\text { Recearch }\end{array}$ & KJ010I, KJ350I & & & 35.81 & 35.81 & 36.40 & 32.90 & & & & 3.50 \\
\hline Grand Challenge Applications & KJ010I, KJ3501 & & 11.20 & 10.00 & 10.00 & 10.00 & 10.00 & & & & \\
\hline Grand Challenge Applications (continued) & KJ0101, KJ3501 & & & & & & & & & & \\
\hline DOE2000 NC & KJ0102 & & & 0.18 & 0.18 & 5.00 & & & & 5.00 & \\
\hline National Energy Research Scientific Computing Center & KJ0102, KJ3501 & & 34.00 & 30.30 & 30.30 & 29.76 & 29.76 & & & & \\
\hline ESnet & KJ0102, KJ3501 & & 15.00 & 12.64 & 12.64 & 14.79 & & 14.79 & & & \\
\hline ESnet (continued) & $\mathrm{KJ} 0102, \mathrm{KJ} 3501$ & & & & & & & & & & \\
\hline Collaboration & KP 05 & & 2.00 & & & & & & & & \\
\hline Information Infraservices & KC 0702 & & 2.00 & & & & & & & & \\
\hline National Challenges & KC 0702 & NASA, EPA, & 0.50 & & & & & & & & \\
\hline Education Programs & $\mathrm{KC} 0701$ & & 4.40 & & & & & & & & \\
\hline Basic Research for Applied Mathematics Research & KC 0701 & NSF & 16.50 & & & & & & & & \\
\hline & & Totals: & 122.35 & 109.69 & 109.69 & 124.56 & 93.29 & 14.79 & & 12.98 & 3.50 \\
\hline
\end{tabular}




\section{Department of Energy}

\begin{tabular}{|c|c|c|c|c|c|c|c|c|c|c|c|c|c|c|}
\hline \multirow[b]{2}{*}{ Program Activity } & \multicolumn{4}{|c|}{ Budget (BA, \$ M) } & \multicolumn{5}{|c|}{ HPCC PCAs by 1997 Pres. Request } & \multicolumn{5}{|c|}{ HPCC PCAs by 1996 Estimated } \\
\hline & $\begin{array}{l}\text { FY } 95 \\
\text { Actual } \\
\end{array}$ & $\begin{array}{l}\text { FY } 96 \\
\text { Pres. }\end{array}$ & $\begin{array}{l}\text { FY } 96 \\
\text { Est. }\end{array}$ & $\begin{array}{l}\text { FY } 97 \\
\text { Rqst. }\end{array}$ & HECC & LSN & HCS & HuCS & ETHR & HECC & LSN & HCS & HuCS & ETHR \\
\hline $\begin{array}{l}\text { Advanced Computational Testing and Simulation } \\
\text { Besearch }\end{array}$ & & 35.81 & 35.81 & 36.40 & 32.90 & & & & 3.50 & 31.81 & & & & 4.00 \\
\hline $\begin{array}{l}\text { Advanced Computational Testing and Simulation } \\
\text { Recearch (continued) }\end{array}$ & & & & & & & & & & & & & & \\
\hline Grand Challenge Applications & 11.20 & 10.00 & 10.00 & 10.00 & 10,00 & & & & & 10.00 & & & & \\
\hline Grand Challenge Applications (continued) & & & & & & & & & & & & & & \\
\hline National Collaboratory Research & & 8.38 & 8.38 & 7.98 & & & & 7.98 & & & & & 8.38 & \\
\hline DOE2000 ACTS & & & & 5.00 & 5.00 & & & & & & & & & \\
\hline DOE2000 NC & & 0.18 & 0.18 & 5.00 & & & & 5.00 & & & & & 0.18 & \\
\hline National Energy Research Scientific Computing Center & 34.00 & 30.30 & 30.30 & 29.76 & 29.76 & & & & & 30.30 & & & & \\
\hline ESnet & 15.00 & 12.64 & 12.64 & 14.79 & & 14.79 & & & & & 12.64 & & & \\
\hline ESnet (continued) & & & & & & & & & & & & & & \\
\hline High Performance Computing Resource Providers & 12.20 & 12.39 & 12.39 & 15.63 & 15.63 & & & & & 12.39 & & & & \\
\hline Gigabit Research and Development & 2.00 & & & & & & & & & & & & & \\
\hline Software Components and Tools & 13.25 & & & & & & & & & & & & & \\
\hline High Performance Computer Systems & 9.30 & & & & & & & & & & & & & \\
\hline Collaboration & 2.00 & & & & & & & & & & & & & \\
\hline Information Infraservices & 2.00 & & & & & & & & & & & & & \\
\hline National Challenges & 0.50 & & & & & & & & & & & & & \\
\hline Education Programs & 4.40 & & & & & & & & & & & & & \\
\hline Basic Research for Applied Mathematics Research & 16.50 & & & & & & & & & & & & & \\
\hline Totals: & 122.35 & 109.69 & 109.69 & 124.56 & 93.29 & 14.79 & & 12.98 & 3.50 & 84.49 & 12.64 & & 8.56 & 4.00 \\
\hline
\end{tabular}


This activity has three fundamental research components: Applied Mathematics, Computer Science, and Software Tools. In addition, this activity supports education Thich are focused on educating the next generation of computational scientists.

The applied mathematics research component develops theory, algorithms, and tools for enabling the solution of large scientific and engineering problems. This component supports leading-edge research at ten DOE laboratories and over 30 universities. Applied mathematicians and computational scientists supported by the basic research program are also active in Grand Challenge projects and other multidisciplinary research projects. Supported laboratory and university researchers meet every two years at a workshop designed to foster collaborations and idea exchanges.

The computer science and software tools component of this effort focuses on developing advanced software to facilitate the use of high performance systems to scientific problems. Efforts range from efficient operating systems and 1/O software for MPP's, to frameworks for isolating application codes from the underlying hardware details, to tools for monitoring the performance of scientific applications. In addition, this component supports efforts to improve the management, visualization, and understanding of the results of high end computations.

The education programs focus on engaging and training students, teachers, and faculty from middle and high school through graduate school in computing, networking, and computational science, by leveraging the large DOE investment in these areas at universities and the national laboratories.

The Computational Science Graduate Fellowship (CSGF) Program supports over 50 doctoral students in computational science and engineering at selected universities. Participating fellows spend at least one summer working at a DOE laboratory in the area of their dissertation. (continued)

\section{Milestone}

Changes

FY 1995 Actual Milestones

\section{FY 1996 Estimated Milestones}

Relocated the Lawrence Livermore National

Laboratory (LLNL) applied mathematics group to

Lawrence Berkeley National Laboratory (LBNL) as part of the NERSC relocation.

Computational scientists at Los Alamos National Laboratory (LANL) and LBNL, working with university partners and a commercial combustion firm, released a code based on adaptive mesh refinement techniques that code based on adaptive mesh refinement techniq
simulates 3D combustion phenomena in complex geometries, with radiation effects included, accurately enough for the code to be used in the design of commercial low NOx burners.

Applied mathematicians at Argonne National Laboratory (ANL), in partnership with researchers at nearby Northwestern University, set up the Optimization Technology Center (OTC) at Northwestern, in order to enhance laboratory/university collaborations.

On-line services at the OTC enable researchers around the country to access software for solving large-scale optimization and programming problems.

Oak Ridge National Laboratory (ORNL) researchers developed and distributed improved codes for sparse matrix calculations that enabled progress on various Grand Challenge problems.

(continued)

\section{FY 1997 Agency Request Milestones} Reduce support for the Ames Laboratory and Brookhaven National Laboratory applied mathematic programs as part of an overall redirection of funds into more computationally intensive areas.

LBNL and LANL computational combustion

esearchers, along with their university partners, will initiate simulations of internal combustion engines, in collaboration with industry partners.

Continue collaboration between ANL applied mathematicians and the DOE Office of Integrated Analysis and Forecasting on improving the predictive capabilities of the National Energy Modeling System. Initiate research at LANL on the predictability of catastrophic events such as wildfires and earthquakes through the development of modeling and simulation tools.

Strengthen collaborative ties between laboratory and university researchers working on Grand Challenge problems and other multidisciplinary projects importan to the DOE mission. Establish interoperability of

MUSE and CAVE virtual reality technologies to

explore domains of most advantageous use of these two technologies.

(continued)
Budget (\$ M)

FY 95 Act

FY 96 Pres.

FY 96 Est.

35.81

FY 97 Rqst.

36.40

Program

Component

Areas

FY 96 FY 97

LSN

LSN

HCS

HuCS

ETHR

4.00

3.50

Agency Ties

\begin{tabular}{|c|c|}
\hline DARPA & Partner \\
\hline NSF & Partner \\
\hline DOE & \\
\hline NASA & Partner \\
\hline NIH & \\
\hline NSA & \\
\hline NIST & \\
\hline NOAA & \\
\hline EPA & \\
\hline ED & \\
\hline AHCPR & \\
\hline VA & \\
\hline
\end{tabular}

December 20, 1996 


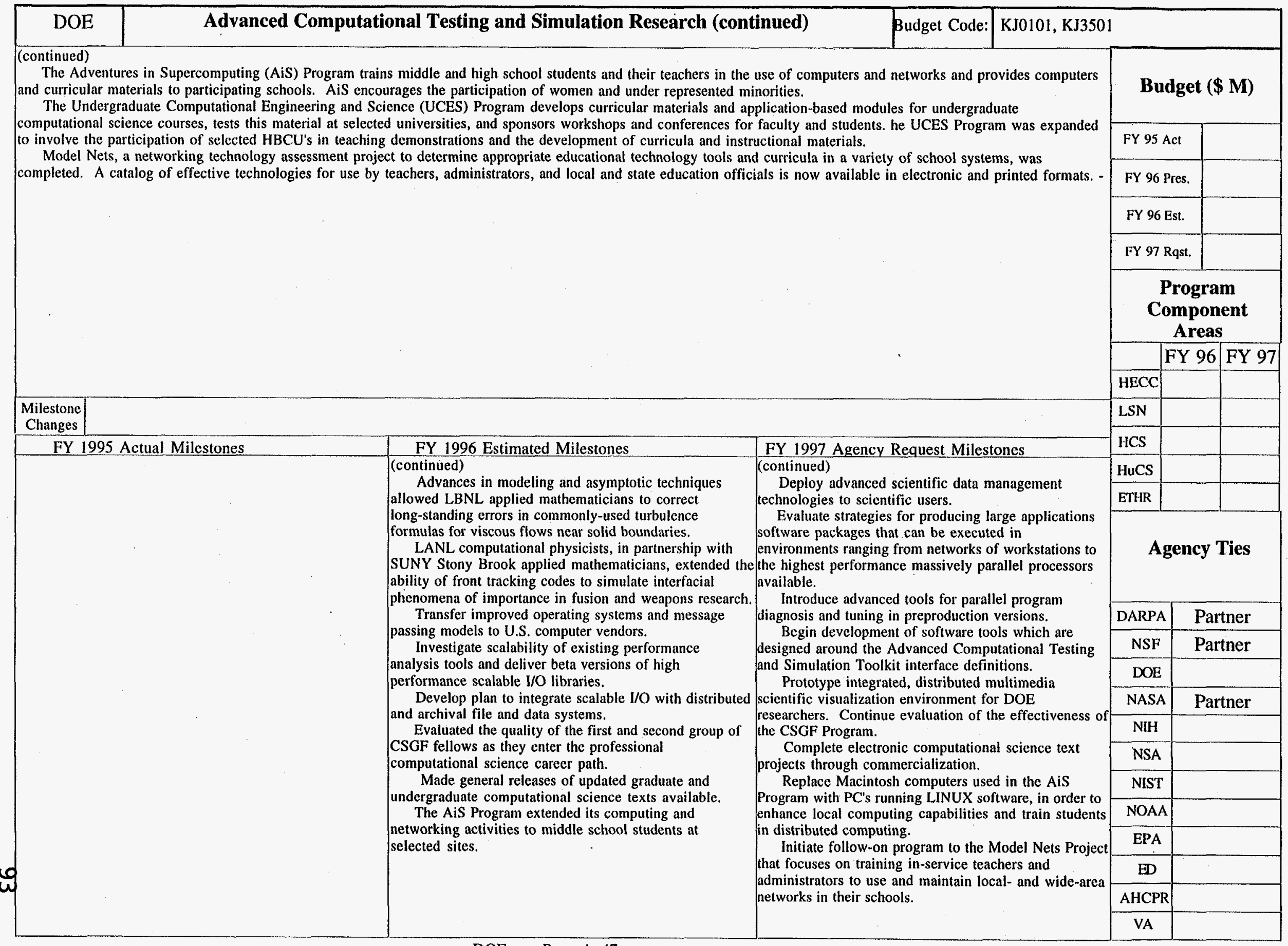


In FY 1992, DOE initiated nine Phase I Grand Challenge projects (GCs) crucial to energy issues. All projects are co-funded by other DOE programs and by industrial partners or other agencies. Participants include DOE laboratories, universities, industry and other HPCC agencies. These projects address the Grand Challenges through the development of advanced algorithms and software and the use of HPCC resources. The selection of these projects was made by a panel including DOE program managers and other HPCC agency participants. Each project undergoes periodic reviews to assess research progress and future plans for continued funding. The projects are:

1. Computational Chemistry (CC) -- parallelize key chemistry codes that permit researchers to study environment problems, using techniques such as self-consistent field (SCF), second order many-body perturbation theory (MP2), and Configuration Interaction (CI) codes;

2. Computational Structural Biology -- develop methods for modeling components of genomes and a parallel programming environment for structural biology;

3. Mathematical Combustion Modeling (MCM) -- develop adaptive parallel algorithms for computational fluid dynamics and apply these methods to key problems in commercial burner design;

4. Quantum Chromodynamics Calculations -- develop lattice gauge algorithms on massively parallel machines for high energy and particle physics applications;

5. Oil Reservoir Modeling -- construct efficient algorithms for parallel systems to model fluid flow through permeable media for better oil recovery methods from wells;

6. The Numerical Tokamak Project -- develop and integrate particle and fluid plasma models on MPPs as part of a study of Tokamak fusion reactors; (continued)

\section{Milestone \\ Changes}

$$
\text { FY } 1995 \text { Actual Milestones }
$$

$\mathrm{CC}$ - developed scalable nonlinear optimization techniques and solution schemes for SCF, MP2 and $\mathrm{Cl}$ codes. Expanded interactions and benchmarking with industrial collaborators.

MCM - extended code to simulate low-speed, 3 combustion flows in complex geometries, such as internal combustion engines.

GCM - applied differential sensitivity analysis and employed a barotropic closure model to resolve large-scale ocean dynamics. Improve strategies for coupling ocean and atmospheric models.

GTR - tested bioremediation and radionuclide decay strategies; incorporated two-phase and three-phase flow with relative permeabilities, additional chemical reactions, and simple mass transfer between phases and components into GCT. Developed a tight-binding molecular dynamics production code for more than 2000 atoms; implemented a full large-system multiple scattering method code on the Paragon in the MPGC.

\section{FY 1996 Estimated Milestones}

Some representative research accomplishments of Phase I GCs in FY_1996 are:

CC -- developed scalable approaches for evaluation of multi-configuration wave functions like

Multi-configuration SCF and CI.

Developed parallel algorithms for analytic SCF second derivatives and MP2 gradients. Integrated scalable input/output techniques into the codes. GCM -- demonstrated successful coupling of atmospheric-ocean-sea ice models on MPPs. GCT -- 3D front-tracking and simple fracture modeling were incorporated into the GCT code in the Groundwater Remediation Grand Challenge. This permitted studies of three-phase flow in

fractured regimes and the role of geostatics to obtain bounding calculations for uncertainties of remediation strategies.

Classical Molecular Dynamics, Tight- Binding

Molecular Dynamics, ab initio Pseudopotential, and Large Scale Multiple Scattering codes were ported to the Intel Paragon XP/S 150.

Unified graphical pre- and post-processing tools for major codes were developed. (continued)
FY 1997 Agency Request Milestones

Evaluate results from completed Phase I GC projects.

Complete Phase II GC applications review and make new awards.

The number of projects supported will be reduced to provide funding of the initiative in Advanced Computational Testing and

Simulation.'

Funding of computational infrastructural component at the DOE's High Performance Computing Resource Centers (HPCRCs) will be

coordinated with this activity.

and

\section{Budget ( $\$$ M)}

\begin{tabular}{|c|c|}
\hline FY 95 Act & 11.20 \\
\hline FY 96 Pres. & 10.00 \\
\hline FY 96 Est. & 10.00 \\
\hline FY 97 Rqst. & 10.00 \\
\hline
\end{tabular}

Program

Component

Areas

\begin{tabular}{|l|c|c|}
\hline & FY 96 & FY 97 \\
\hline HECC & 10.00 & 10.00 \\
\hline LSN & & \\
\hline HCS & & \\
\hline HuCS & & \\
\hline ETHR & & \\
\hline
\end{tabular}

\section{Agency Ties}

\begin{tabular}{|c|c|}
\hline DARPA & \\
\hline NSF & \\
\hline DOE & \\
\hline NASA & \\
\hline NIH & \\
\hline NSA & \\
\hline NIST & \\
\hline NOAA & \\
\hline EPA & \\
\hline ED & \\
\hline AHCPR & \\
\hline VA & \\
\hline
\end{tabular}




\begin{tabular}{|c|c|c|c|}
\hline DOE & Grand Challenge Applications (continued) & Budget Code: & KJ0101, KJ3501 \\
\hline (continued) & . & \\
\hline
\end{tabular}

7. Global Climate Modeling (GCM) -- develop and implement versions of large-scale atmosphere and ocean general circulation models for MPPs;

Groundwater Transport and Remediation (GTR) -- design and implement a multiphase groundwater transport code with interface tracking, fracture flow,

microtransport; and

9. First Principles Simulation of Materials Properties -- develop scalable parallel algorithms for performing local density approximation simulations of materials to novel properties for the Materials Properties Grand Challenge (MPGC).

At the end of FY 1996, Phase I of the GC program was terminated. An RFP was published for Phase II of the program with project starts early in FY 1997. The intent and grant procedures of Phase II are similar to Phase I but the projects now incorporate a infrastructural component to insure that the projects have the computational resources to complete their proposed goals.

\section{Milestone}

Changes

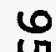

\begin{tabular}{|l|}
\hline FY 1995 Actual Milestones \\
\hline
\end{tabular}

FY 1996 Estimated Milestones

(continued)

MCM -- Incorporated radiation effects into codes

that simulate the behavior of low NOx commercial

burners and design algorithms for simulating high

speed combustion processes in realistic 3D

geometries.

RFP published for Phase II Grand Challenge

Projects and technical review was begun. Thirty-six

applications were submitted.

\section{FY 1997 Agency Request Milestones}

\begin{tabular}{|c|c|}
\hline LSN & \\
\hline HCS & \\
\hline HuCs & \\
\hline ETHR & \\
\hline \multicolumn{2}{|c|}{ Agency Ties } \\
\hline DARPA & \\
\hline NSF & \\
\hline $\mathrm{DOE}$ & \\
\hline NASA & \\
\hline $\mathrm{NIH}$ & \\
\hline NSA & \\
\hline NIST & \\
\hline NOAA & \\
\hline EPA & \\
\hline ED & \\
\hline AHCPR & \\
\hline $\mathrm{VA}$ & \\
\hline
\end{tabular}

December 20, 1996 
The National Collaboratory Research is structured to develop the technologies and tools that will enable scientists and engineers to easily collaborate across geographic boundaries and to interact cooperatively in common problem solving and experimental activities. Elements of this research address the basic technology and services that underlie a number of national challenge applications including energy demand management and remote environmental monitoring.

Information Infrastructure technology R\&D is conducted in information navigation and analysis tools, in hierarchical distributed information storage, advanced collaborative multimedia environments and in information surety.

Networking research activities include security (such as providing secure information retrieval and search mechanisms and interoperable authentications realms), secure software distribution intelligent user interfaces for accessing the network, interoperable interactive multimedia systems, high speed LANs and WANs, telepresence, protocols and services to support energy demand and supply management as well as collaborative work environments.

Part of this research activity will investigate and develop immersive environment (e.g. CAVE) use of high speed low latency networks and protocols. This activity includes evaluation and use of ATM capabilities (e.g., Quality Of Service, signaling) to support advanced applications as well as provide interagency collaborative environments.

\section{Milestone}

Changes

FY 1995 Actual Milestones

\section{FY 1996 Estimated Milestones}

Defined a security architecture that provides transparent and easily administered security services.

Software developed to allow remote access to Advanced Light Source (ALS) experiments across ESnet was deployed in version 1.0 release.

Developed a prototype system for monitoring power quality over the Internet and for providing associated energy services.
FY 1997 Agency Request Milestones

Integrate existing collaborative tools into the National Collaboratory Framework. Virtual real advancement to allow a CAVE to CAVE interaction of at least twenty minutes.

Generalize and package the component developed for the on-line access of the ALS for general use in remote collaborative access of facilities and instruments.

First release of LabSpace software--an integrated media server for providing hypermedia indexing and information navigation and discovery, n-way multipoint media services and parallel interactive serving.

Prototype a system allowing multiple commercial building monitoring and operations from a single remotely-sited control room.

Prototype, on a small scale, residential energy demand management using information infrastructure (i.e., the Internet)

Make the adaptive secure high speed communications library for hi-end systems available for evaluation and use.

Prototype the Secure Software distribution system.
Budget ( $\$ \mathbf{M})$

\begin{tabular}{|c|c|}
\hline FY 95 Act & \\
\hline FY 96 Pres. & 8.38 \\
\hline FY 96 Est. & 8.38 \\
\hline FY 97 Rqst. & 7.98 \\
\hline
\end{tabular}

Program

Component

Areas

\begin{tabular}{|l|l|l|}
\hline & FY 96 & FY 97 \\
\hline HECC & & \\
\hline LSN & & \\
\hline HCS & & \\
\hline HuCS & 8.38 & 7.98 \\
\hline ETHR & & \\
\hline
\end{tabular}

Agency Ties

\begin{tabular}{|r|c|}
\hline DARPA & Partner \\
\hline NSF & Partner \\
\hline DOE & \\
\hline NASA & \\
\hline NIH & \\
\hline NSA & \\
\hline NIST & \\
\hline NOAA & \\
\hline EPA & \\
\hline ED & \\
\hline AHCPR & \\
\hline VA & \\
\hline
\end{tabular}

December 20, 1996 


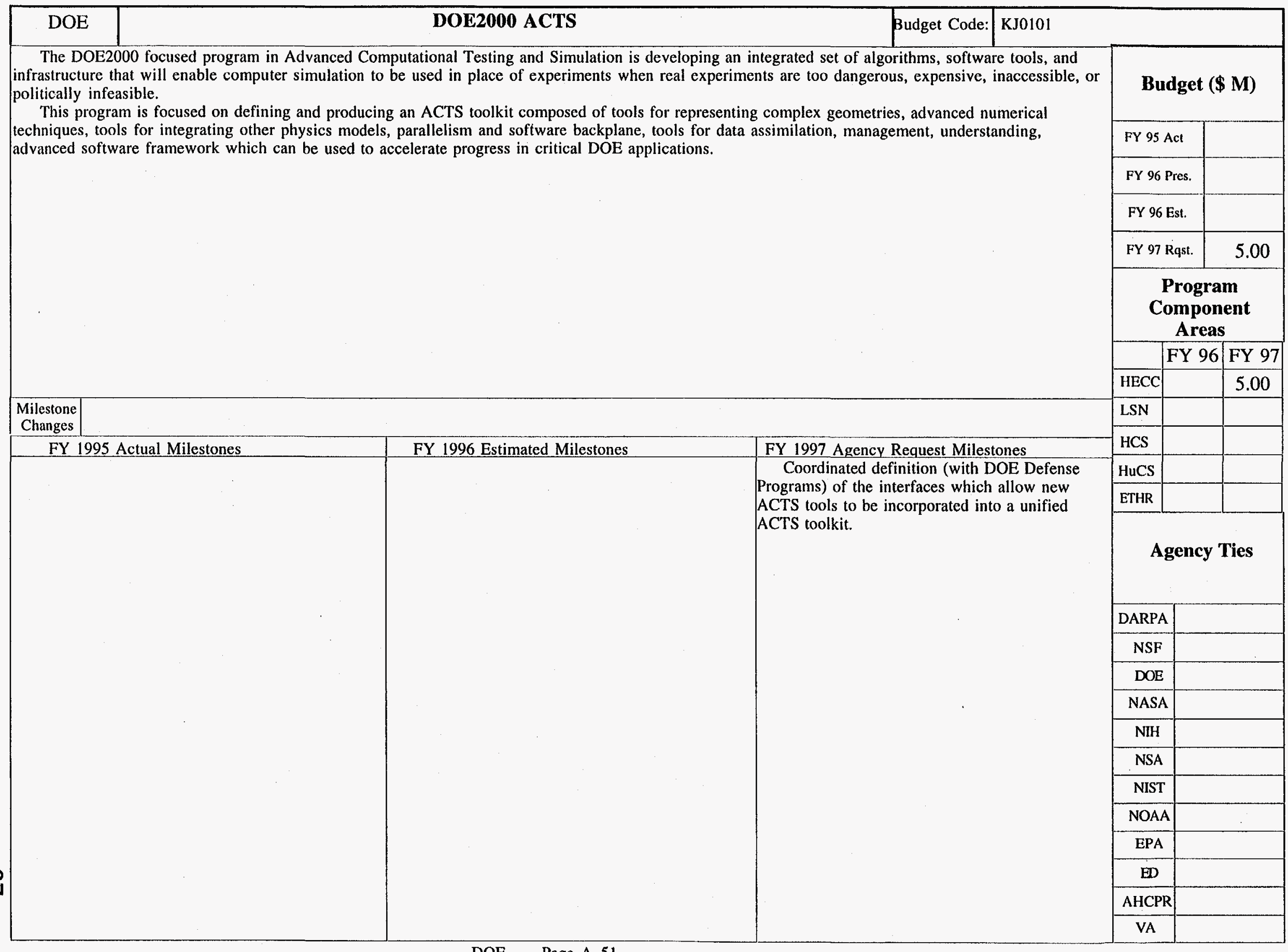


The DOE2000 focused program in National Collaboratories (NC) is developing a set of tools and capabilities which will permit scientists and engineers $\infty$ working at different DOE and other facilities to collaborate on solving problems as easily as if they were in the same building. The program supports research in the tools which a virtual laboratory requires: Collaborative tools; Information surety (authentication + security), and High-performance networking and one pilot implementations of these tools in partnership with other DOE programs.

\begin{tabular}{|c|c|}
\hline $\begin{array}{c}\text { Milestone } \\
\text { Changes }\end{array}$ & FY 1996 Estimated Milestones \\
\hline FY 1995 Actual Milestones & $\begin{array}{c}\text { Conduct workshop on DOE2000 NC technologies } \\
\text { and initiate planning for integrated technology } \\
\text { development program and pilot projects. }\end{array}$ \\
\hline
\end{tabular}

FY 1997 Agency Request Milestones Initiate R\&D efforts in critical NC technologies.

Select 2 NC pilot projects from partnership prospectuses from other Offices in DOE.

\section{Budget (\$ M)}

\begin{tabular}{|c|c|}
\hline FY 95 Act & \\
\hline FY 96 Pres. & 0.18 \\
\hline FY 96 Est. & 0.18 \\
\hline FY 97 Rqst. & 5.00 \\
\hline
\end{tabular}

Program

Component

Areas

FY $9 6 \longdiv { \text { FY } 9 7 }$

\begin{tabular}{|l|l|l|}
\hline HECC & & \\
\hline LSN & & \\
\hline HCS & & \\
\hline HuCS & 0.18 & 5.00 \\
\hline ETHR & & \\
\hline
\end{tabular}

\section{Agency Ties}

\begin{tabular}{|c|c|}
\hline DARPA & \\
\hline NSF & \\
\hline DOE & \\
\hline NASA & \\
\hline NIH & \\
\hline NSA & \\
\hline NIST & \\
\hline NOAA & \\
\hline EPA & \\
\hline ED & \\
\hline AHCPR & \\
\hline VA & \\
\hline
\end{tabular}


This activity provides funding for equipment and personnel for the Scientific Applications effort at the National Energy Research Scientific Computing Center (NERSC). This effort provides high performance computing resources for investigators supported by the Energy Research often through collaboration with the NERSC staff. Mission areas of Energy Research include: Material Sciences; Chemistry; Geosciences; Biosciences; Engineering; Health and Environmental Research; High Energy and Nuclear Physics; Fusion Energy; and Mathematical, Information and Computational Science.

The Center serves more than 4,000 users working on about 700 projects, of which about $30 \%$ are university based, $65 \%$ are in National Laboratories, and 5\% in industry. In FY 1996 NERSC operated 4 CRAY computers: a C-90 with 16 processors, with 256 Million words (Mw) of memory, a CRAY-2 with 8 processors with $128 \mathrm{Mw}$, a CRAY 2 with 4 processors and $128 \mathrm{Mw}$, and a J-90 with 32 processors and $512 \mathrm{Mw}$, and the National Education Supercomputer, single processor CRAY XMP donated by Cray Research, which is available over the Internet to high schools for educational programs.

The operating philosophy is not to continue to operate NERSC as a first class production computing facility as it successfully has for the last two decades, but to an interactive Center whose staff will collaborate with DOE/ER scientists and engineers to enable them to accomplish their missions through effective use of the Centers new computing systems with novel architectures.

\section{Milestone \\ Changes}

FY 1995 Actual Milestones

Implemented massively parallel computer in the production environment.

Delivered 576,000 Computer Resource Units

(CRUs) to ER Programs.

Supplied an additional 3 Terabytes of archival storage to ER programs.

The Special Parallel Processing Program was expanded to 70,000 Computer Resource Units by 4Q95. SPP workshops were held for winning. proposals.

Brought the NSL technology-based archival storage system into limited production use.

Brought up the Portable Batch System (PBS) to provide a uniform batch environment across all major platforms.

\section{FY 1996 Estimated Milestones}

While successfully delivering 600,000 Compute Resource Units (CRUs) in FY_1996 (more than in FY_1995) and an additional $\overline{3}$ Terabytes of archival storage to ER programs, the operating philosophy of NERSC was rewritten, the Center was recompeted, moved, and rebuilt at Lawrence Berkeley National Laboratory in response to a directive to operate the Center in a self sustaining mode for $20 \%$ less funding.

In FY_1996, the Center also acquired new computing systems that are not classical vector

Supercomputers. These systems, J90s with a

shared-memory multi-processor architecture and a T3E with a massively parallel architecture, will be acquired from SGI's Cray Research Division

providing certain performance benchmarks are met.

The new systems will increase the computational resources at the Center by more than $400 \%$ before the end of FY_1997. Also acquisition of Mass Storage will improve this component of the Center's system by an order of magnitude in the same time span.

\section{FY 1997 Agency Request Milestones}

Continue to rebuilt the Center to effectively operate within the new philosophy primarily by hiring scientists and engineers trained in the mission disciplines of DOE Energy Research to work interactively with Energy Research users of the Center.

Install new computing and mass storage systems, bring into operation, and train and otherwise aid Energy Research investigators in effective use of the new Center.

Expand the efforts of the Center to assess and use new computing, collaborating and

communications technologies important to Energy Research.

\section{Budget (\$M)}

\begin{tabular}{|c|c|}
\hline FY 95 Act & 34.00 \\
\hline FY 96 Pres. & 30.30 \\
\hline FY 96 Est. & 30.30 \\
\hline FY 97 Rqst. & 29.76 \\
\hline
\end{tabular}

Program

Component

Areas

FY 96 FY 97

\begin{tabular}{|l|l|l|}
\hline HECC & 30.30 & 29.76 \\
\hline LSN & & \\
\hline HCS & & \\
\hline HUCS & & \\
\hline ETHR & & \\
\hline
\end{tabular}

Agency Ties

\begin{tabular}{|c|c|}
\hline DARPA & \\
\hline NSF & \\
\hline DOE & \\
\hline NASA & \\
\hline NIH & \\
\hline NSA & \\
\hline NIST & \\
\hline NOAA & \\
\hline EPA & \\
\hline ED & \\
\hline AHCPR & \\
\hline VA & \\
\hline
\end{tabular}

December 20, 1996 


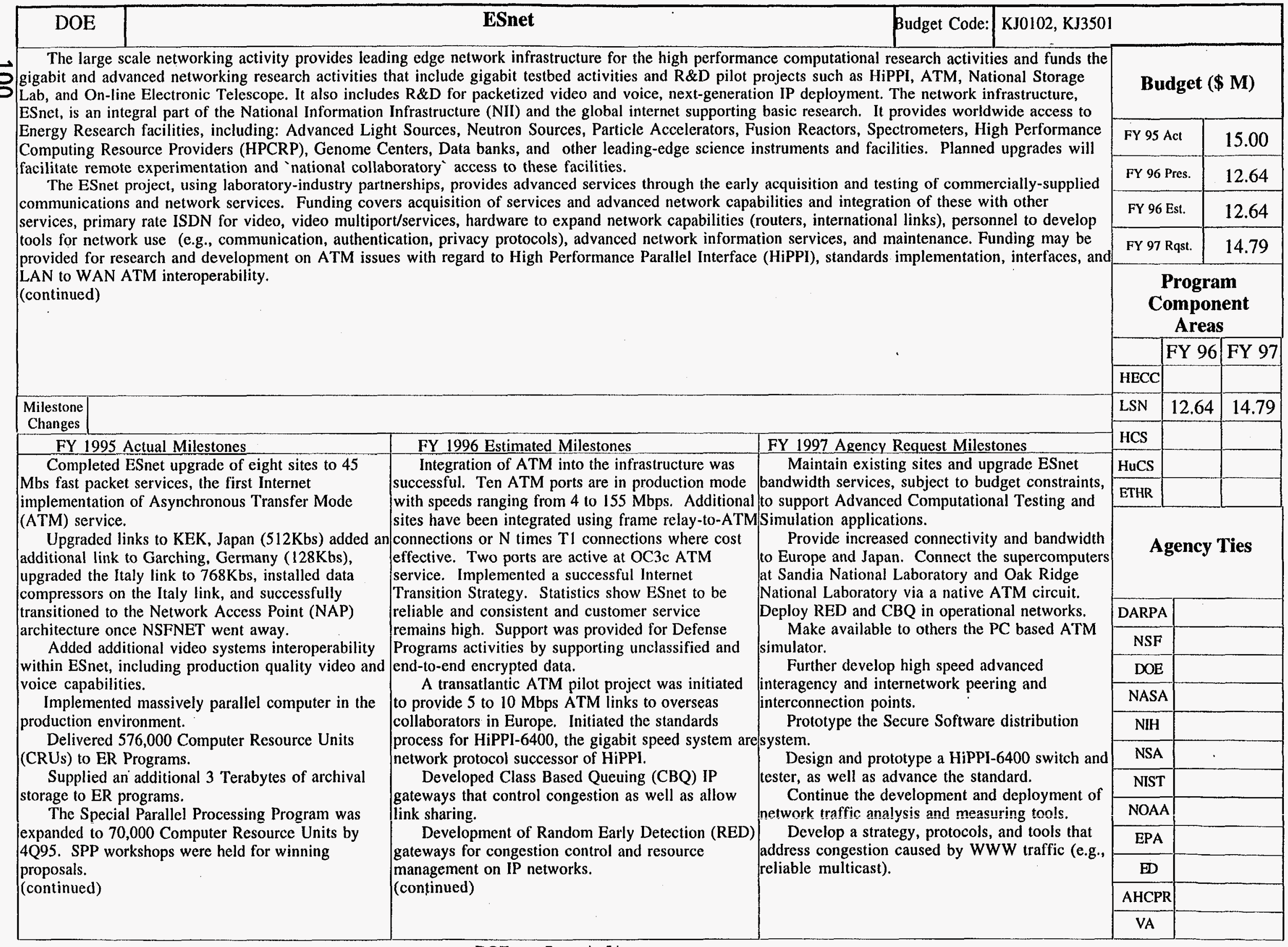




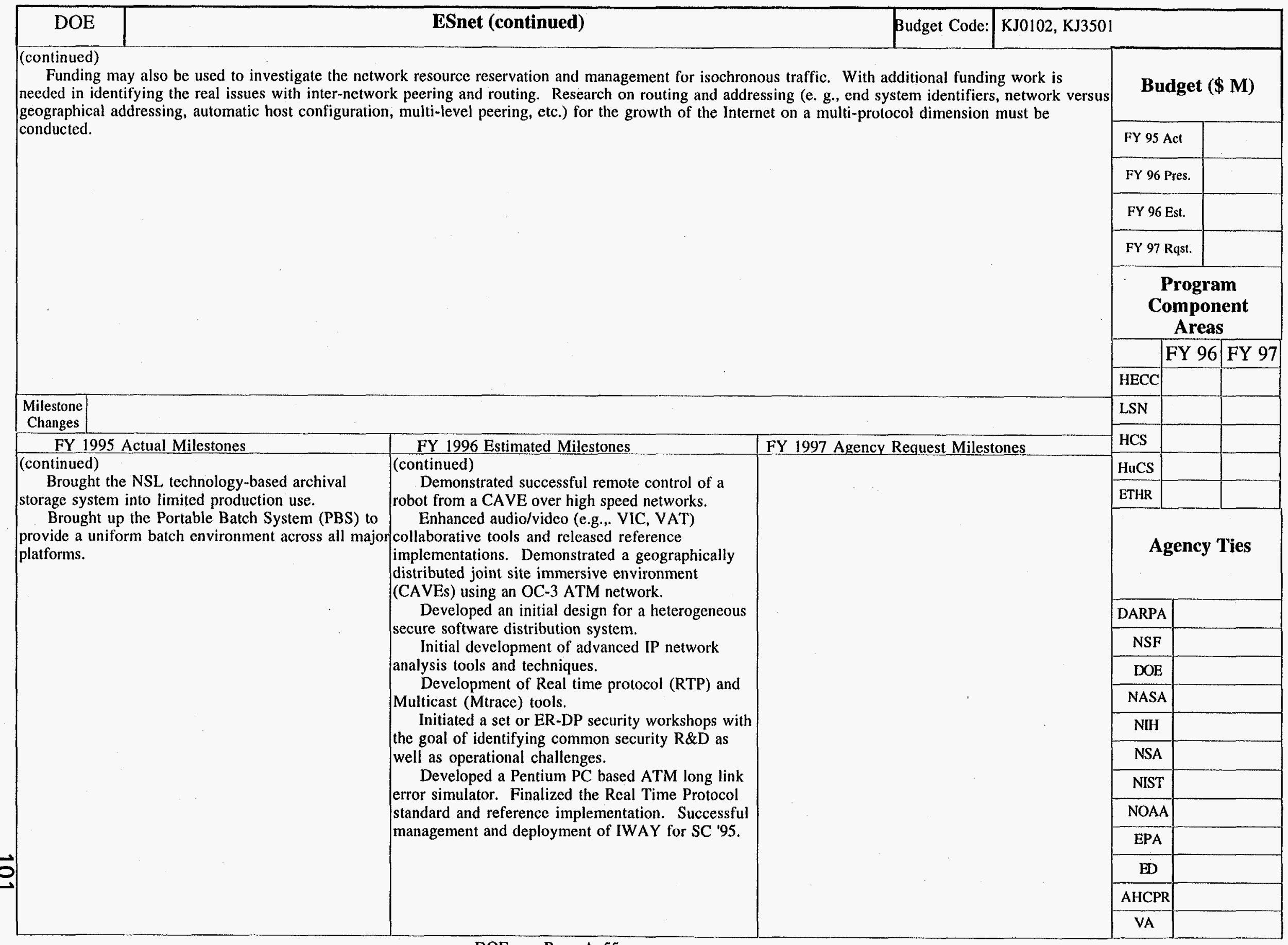




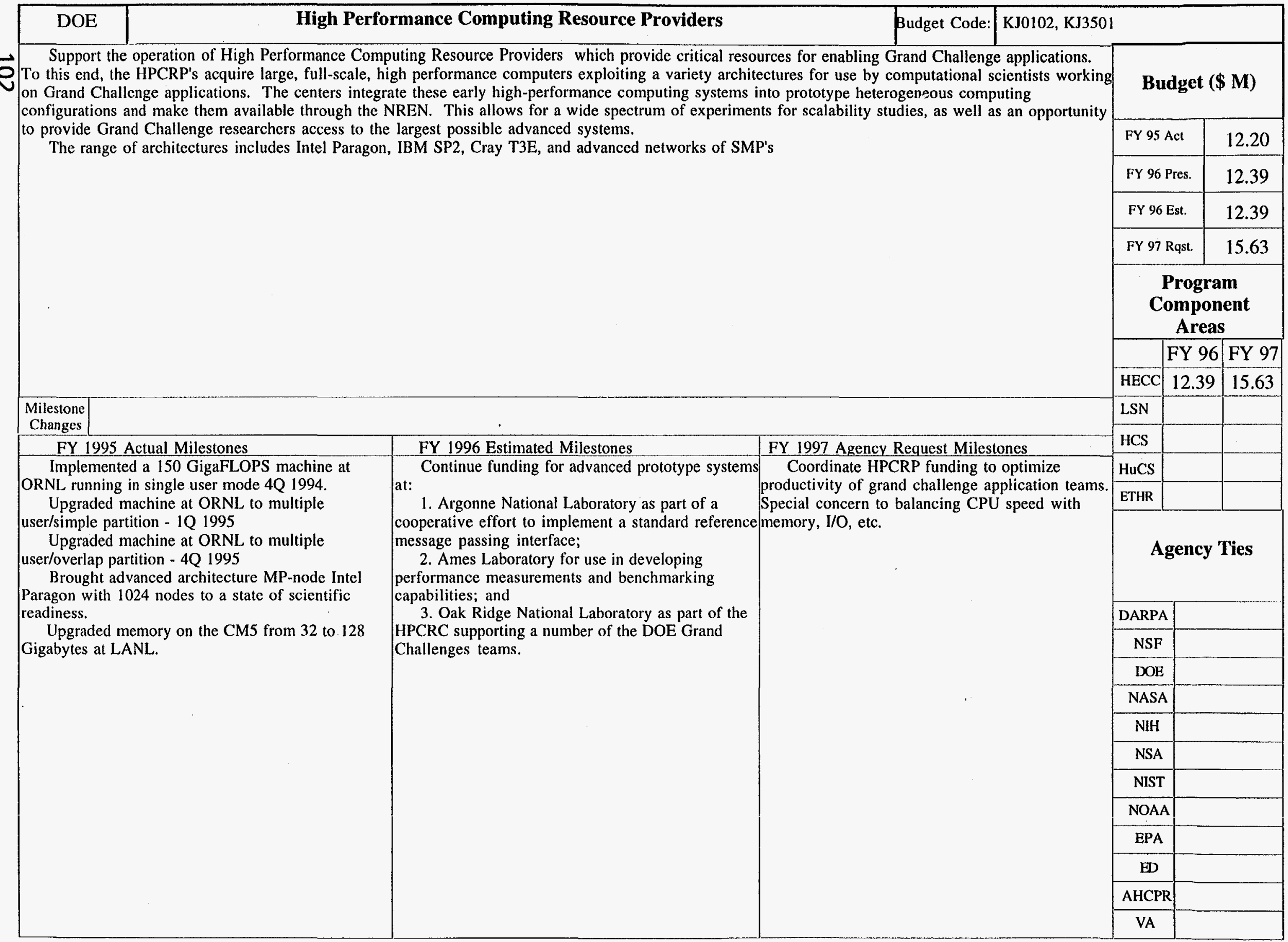


This activity funds the gigabit and advanced networking research activities of the NREN. It includes R\&D in gigabit testbed activities and pilot projects (HiPPI, ATM, National Storage Lab, Online Electronic Telescope, and more), packetized video and voice, collaborative workspace, telecommuting projects, technologies and mechanisms for supporting energy demand and supply management, next-generation IP deployment, and both secure and scalable communication libraries for distributed computing environments.

Funding may be provided for research and development on ATM issues with regard to High Performance Parallel Interface (HiPPI), standards implementation, interfaces, and LAN to WAN ATM interoperability. Funding may also be used to investigate the network resource reservation and management for isochronous traffic. Further work in security (such as providing secure information retrieval and search mechanisms and interoperable

authentications realms), intelligent user interfaces for accessing the network, interoperable video systems, high speed LANs, telecommuting, protocols and services to support energy demand and supply management, and collaborative work environments will be funded. With additional funding work is needed in identifying the real issues with inter-network peering and routing. Research on routing and addressing (e. g., end system identifiers, network versus geographical addressing, automatic host configuration, multi-level peering, etc.) for the growth of the Internet on a multi-protocol dimension must be conducted.

\section{Milestone}

Changes

FY 1995 Actual Milestones

Scalable communications library was completed.

LBL released beta code of collaborative

technologies to ARPA and other agencies for further refinement and development.

Packetization of commercial codec video/audio output was completed and allows ESnet to carry packetized video encapsulated in IP.

Initiated research, through a workshop, on the integration of wide area network and scalable $\mathrm{I} / \mathrm{O}$ techniques and technologies.

FY 1996 Estimated Milestones

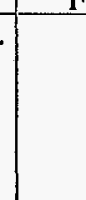

\footnotetext{
$\overrightarrow{\mathrm{\omega}}$
}

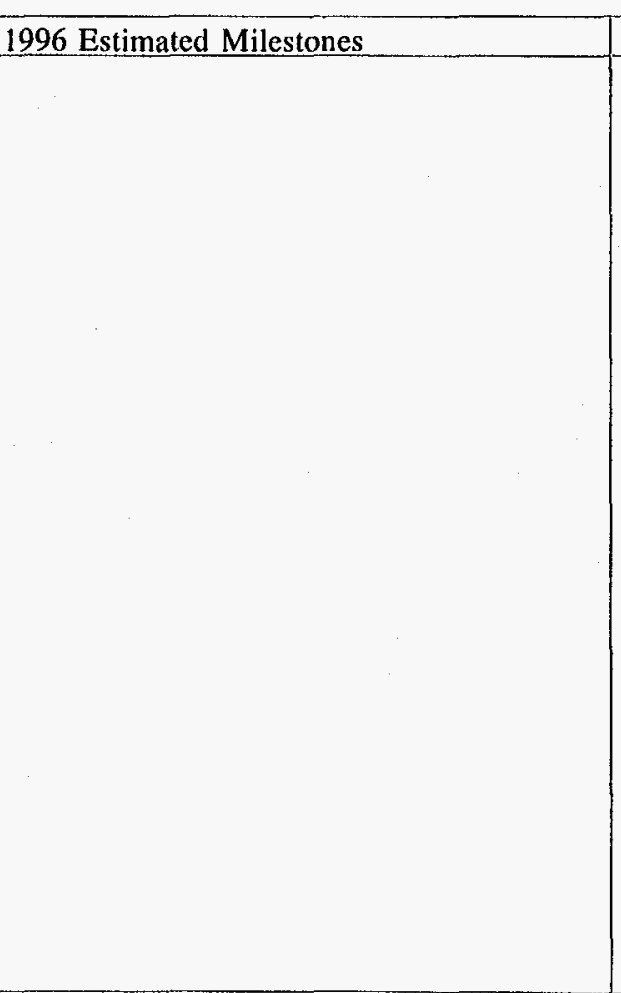

\section{Budget (\$ M)}

\begin{tabular}{|c|c|c|}
\hline \multicolumn{2}{|c|}{ FY $95 \mathrm{Act}$} & 2.00 \\
\hline \multicolumn{2}{|c|}{ FY 96 Pres. } & \\
\hline \multicolumn{2}{|c|}{ FY 96 Est. } & \\
\hline \multicolumn{3}{|c|}{ FY 97 Rqst. } \\
\hline \multicolumn{3}{|c|}{$\begin{array}{c}\text { Program } \\
\text { Component } \\
\text { Areas } \\
\end{array}$} \\
\hline & FY 96 & FY 97 \\
\hline \multicolumn{3}{|c|}{$\mathrm{HECC}$} \\
\hline LSN & & 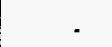 \\
\hline \multicolumn{3}{|l|}{$\mathrm{HCS}$} \\
\hline \multicolumn{3}{|l|}{ HuCS } \\
\hline ETHR & & \\
\hline
\end{tabular}

Agency Ties

\begin{tabular}{|c|c|}
\hline DARPA & Partner \\
\hline NSF & Partner \\
\hline DOE & \\
\hline NASA & Partner \\
\hline NIH & \\
\hline NSA & \\
\hline NIST & \\
\hline NOAA & \\
\hline EPA & \\
\hline ED & \\
\hline AHCPR & \\
\hline VA & \\
\hline
\end{tabular}

December 20, 1996 


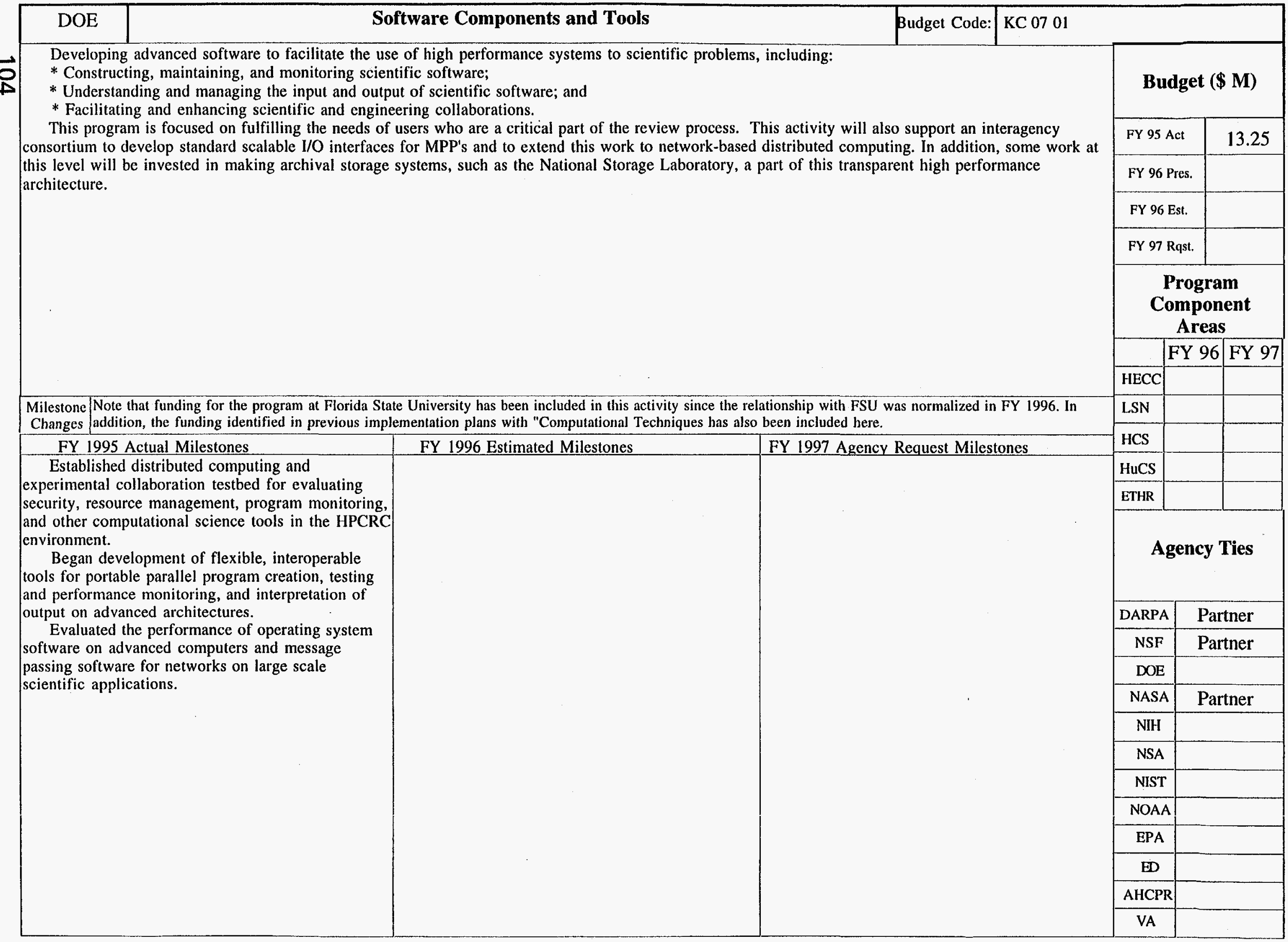




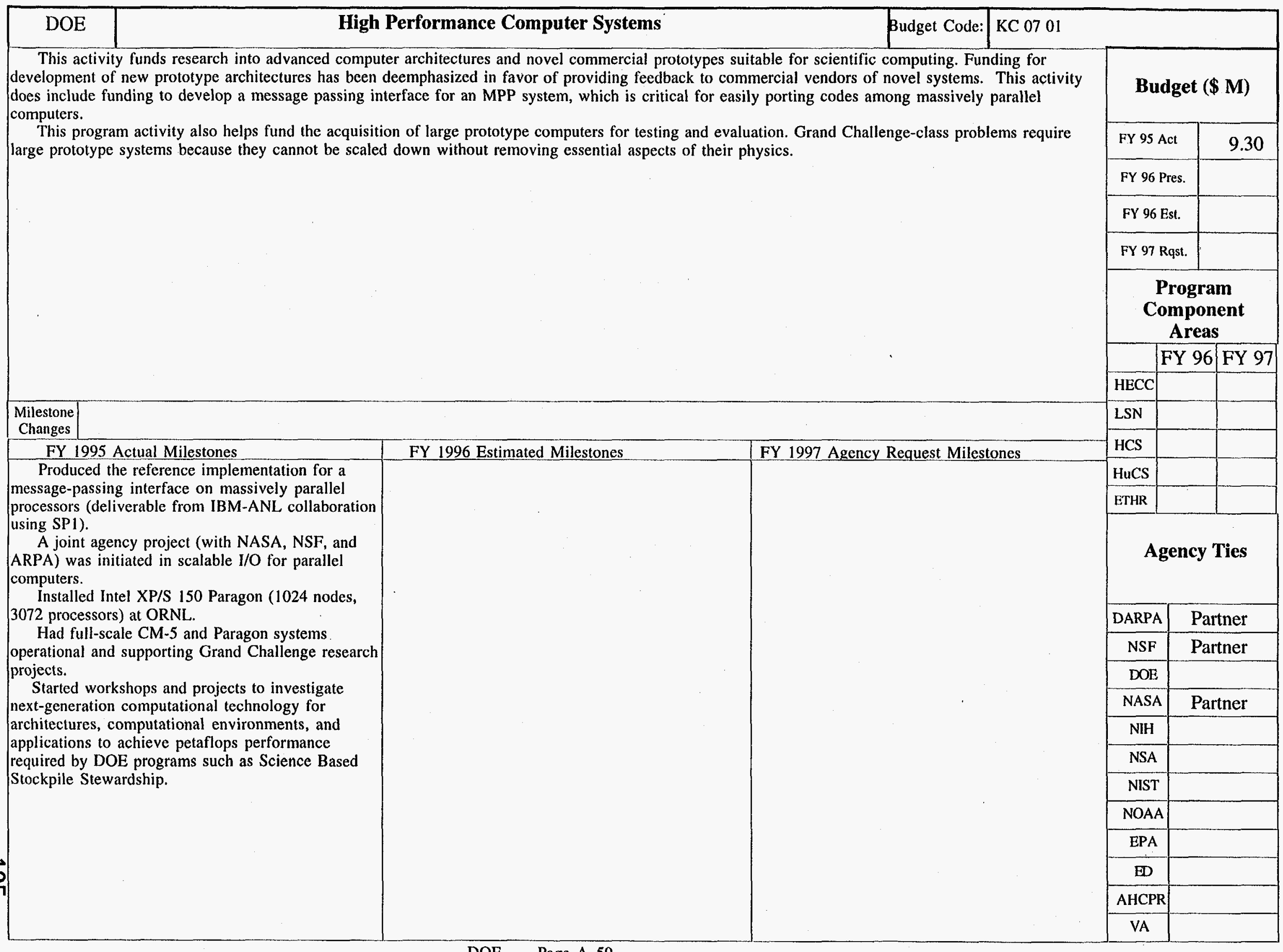




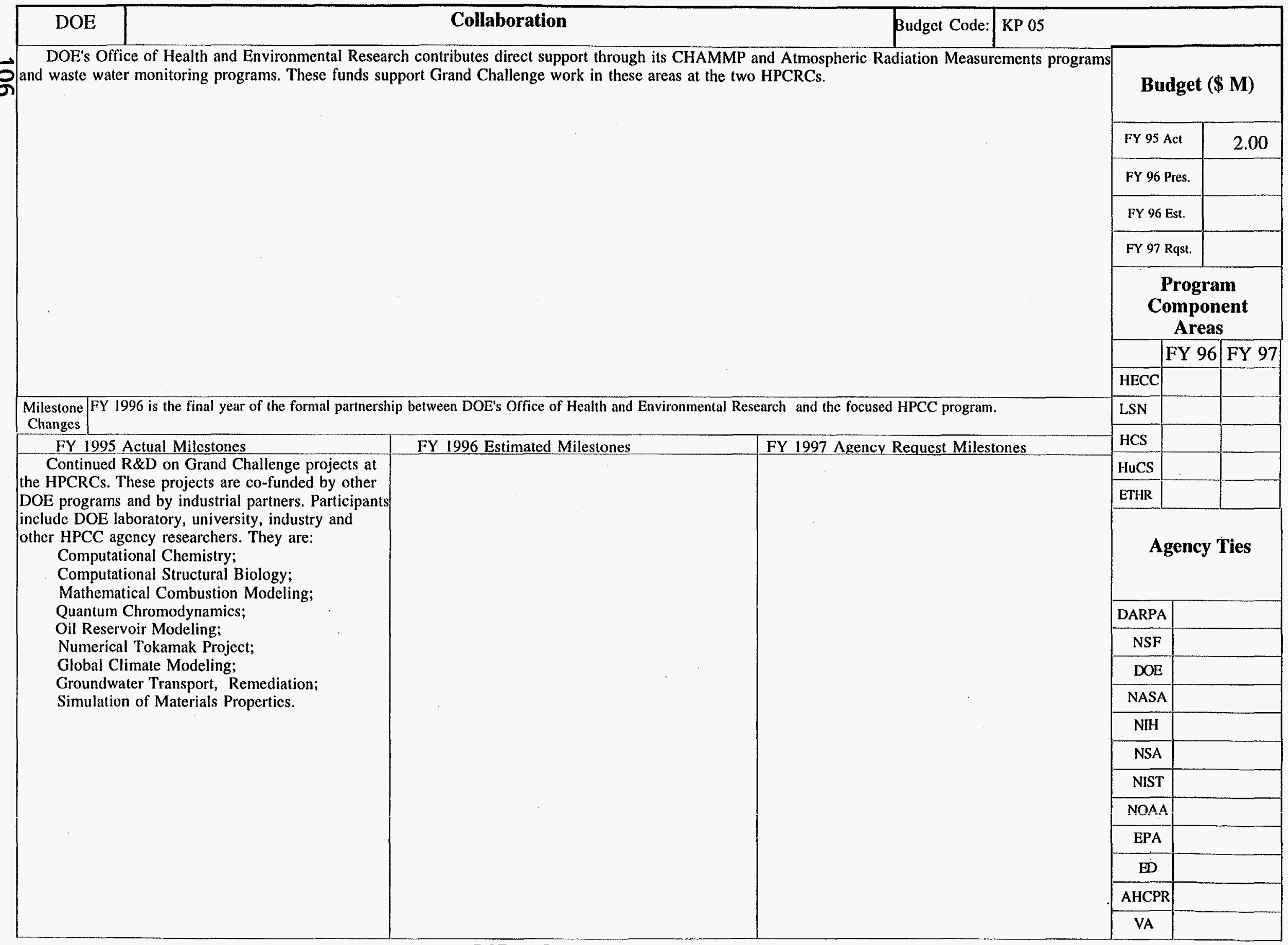


This element supports research in the basic technology and

services that underlie a number of IITA applications including energy demand management and environmental monitoring and waste minimization. The basic services being explored include authentication, privacy and security, distributed resource management, and distributed collaboration tools.

Much of this work is done in close cooperation with U.S. computer manufacturers and potential users in industry.

FY 1995 Actual Milestones

FY 1997 Agency Request Milestones

Established security (smart cards, authenti
etc.) for remote access to resources, such as

libraries, digital and object databases, video

conferences, robots in hazardous areas, etc.

Developed underlying technologies that are

required to implement an energy demand and supply

management National Challenge in close

collaboration with U.S. power utility companies.

FY 1996 Estimated Milestones

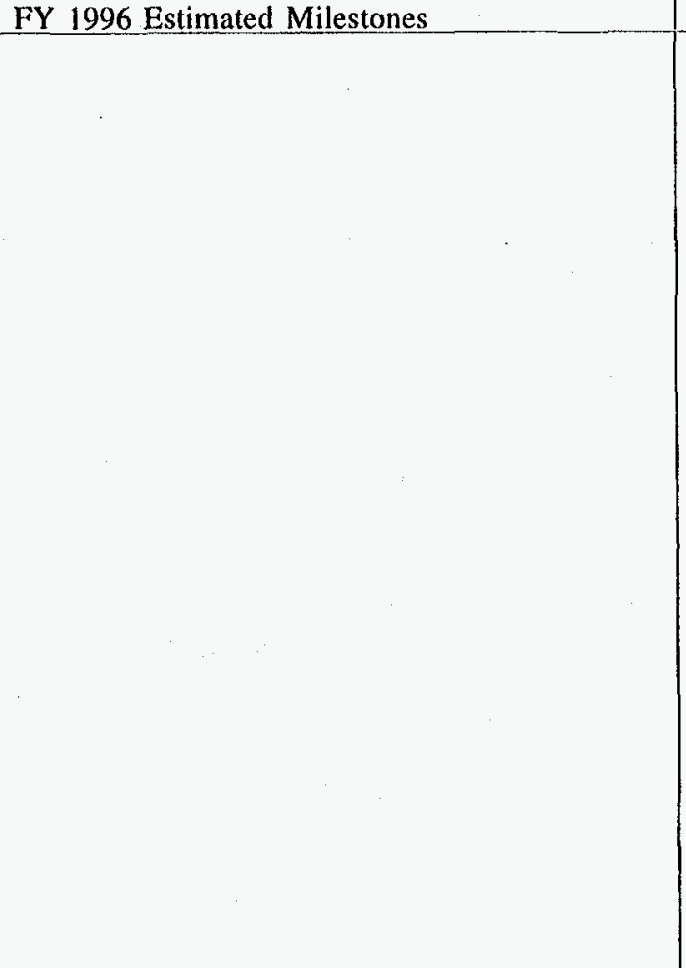

Budget (\$ M)

\begin{tabular}{|c|c|c|}
\hline \multicolumn{2}{|c|}{ FY 95 Act } & 2.00 \\
\hline \multicolumn{2}{|c|}{ FY 96 Pres. } & \\
\hline \multicolumn{2}{|c|}{ FY 96 Est. } & \\
\hline \multicolumn{2}{|c|}{ FY 97 Rqst. } & \\
\hline \multicolumn{3}{|c|}{$\begin{array}{c}\text { Program } \\
\text { Component } \\
\text { Areas }\end{array}$} \\
\hline & FY 96 & FY 97 \\
\hline HECC & & \\
\hline LSN & & \\
\hline HCS & & \\
\hline HuCS & & \\
\hline ETHR & & \\
\hline
\end{tabular}

Agency Ties

\begin{tabular}{|c|c|}
\hline DARPA & Partner \\
\hline NSF & \\
\hline DOE & \\
\hline NASA & \\
\hline NIH & \\
\hline NSA & \\
\hline NIST & \\
\hline NOAA & \\
\hline EPA & Partner \\
\hline ED & User \\
\hline AHCPR & \\
\hline VA & \\
\hline
\end{tabular}

December 20, 1996 


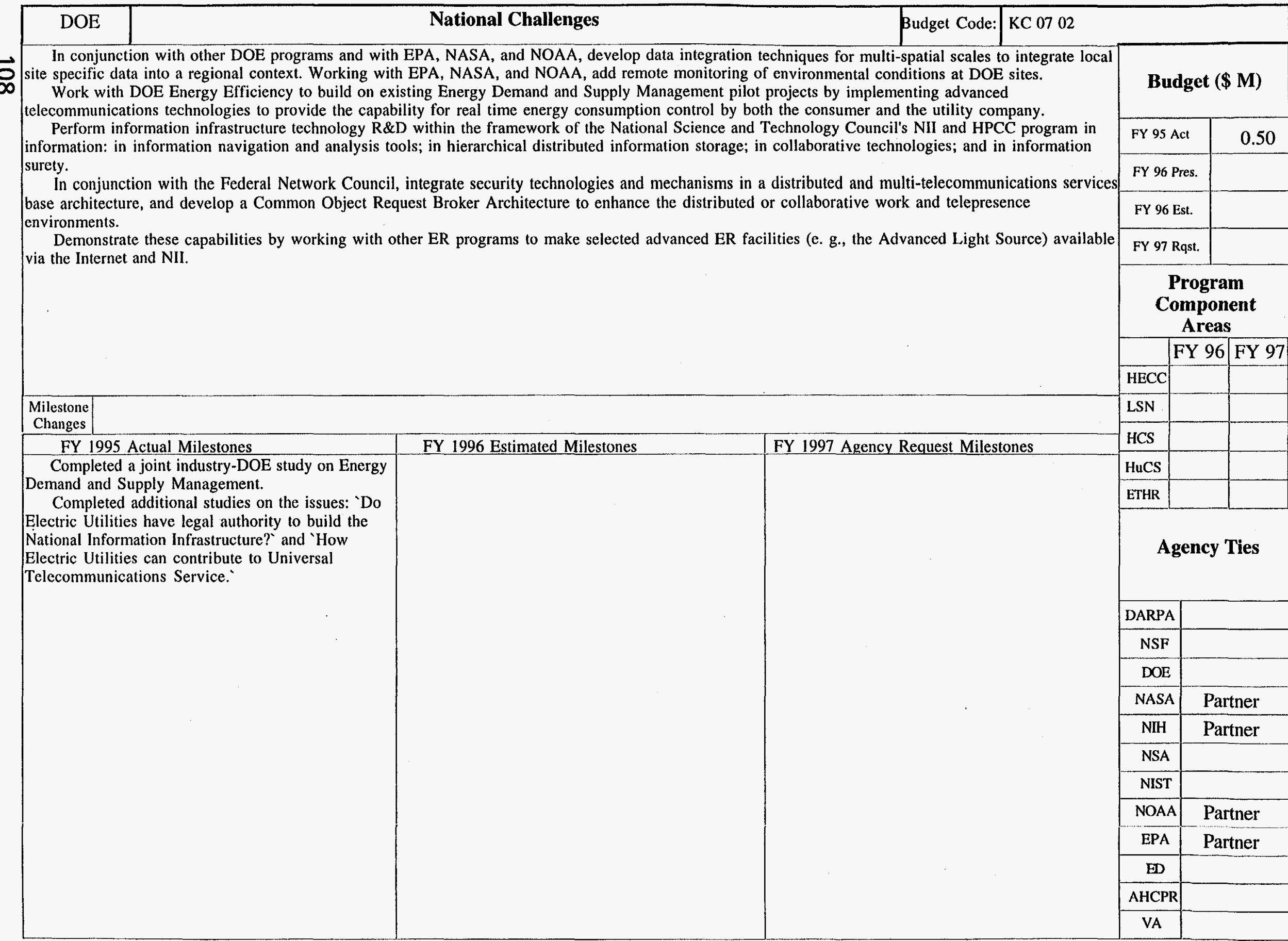




\begin{tabular}{|c|c|c|c|}
\hline DOE & Education Programs & Budget Code: & KC 07 01 \\
\hline
\end{tabular}

The Computational Science Graduate Fellowship program supports over 50 doctoral students in computational science and engineering.

In addition, support is provided for computational science education at various levels where opportunities for significant advancement are anticipated.

Adventures in Supercomputing (AiS) trains in-service teachers in the use of computers and networks. AiS also encourages the participation of women and minorities in computational science.

The National High School Honors Program provides summer enrichment in supercomputing for gifted high school students.

Textbook projects develop instructional materials for undergraduate and graduate courses in computational science.

A curriculum enhancement project is developing approaches for effecting permanent changes in university curricula at both the undergraduate and graduate levels. This activity also addresses the issue of engaging university administrators in the process of advocating computational science and engineering education.

Milestone

Changes

FY 1995 Actual Milestones

Evaluated quality of first group of fellows as they enter the professional computational science career path.

Made second general release of electronic computational science text available.

Continued AiS program at reduced level, but extend computing and networking

activities from high school students to middle school students at selected sites.

Produced a science textbook, using techniques developed in producing the

computational science textbook.

Held a workshop on the role of schools of

education in computational science education.

Initiated networking technology assessmen

project to determine appropriate educational

technology tools and curricula for a wide variety of

schools and to produce a catalog of effective

technologies for use by teachers, administrators and

local and state education officials.

\section{FY 1996 Estimated Milestones}

Moved to the ACTS research activity

\section{FY 1997 Agency Request Milestones}

Budget (\$ M)

\begin{tabular}{|l|l|}
\hline FY 95 Act & 4.40 \\
\hline FY 96 Pres. & \\
\hline FY 96 Est. & \\
\hline FY 97 Rqst. & \\
\hline
\end{tabular}

Program

Component

Areas

FY 96 FY 97

\section{(19)}

L

HCS

\begin{tabular}{l} 
HuC \\
\hline ET
\end{tabular}

\section{Agency Ties}

\begin{tabular}{|c|c|}
\hline DARPA & \\
\hline NSF & \\
\hline DOE & \\
\hline NASA & \\
\hline NIH & \\
\hline NSA & \\
\hline NIST & \\
\hline NOAA & \\
\hline EPA & \\
\hline ED & \\
\hline AHCPR & \\
\hline VA & \\
\hline
\end{tabular}

December 20, 1996 


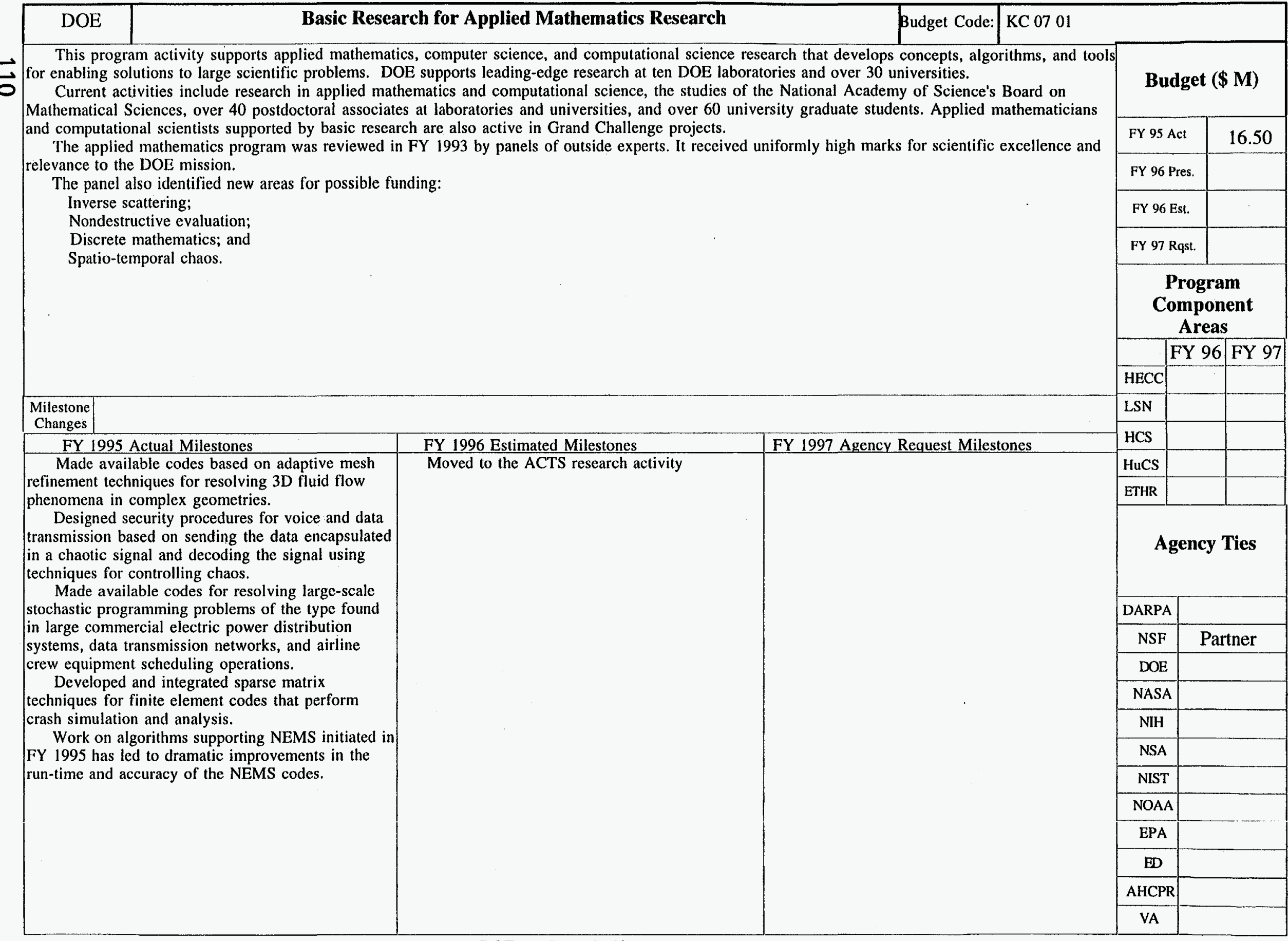




\section{National Aeronautics and Space Administration}

NASA's primary role in the HPCC Program includes leading the development of applications software and algorithms for scalable parallel computing systems that will increase system performance to the sustained teraFLOPS (trillion floating point operations per second) level for NASA applications. As HPCC technologies are developed, NASA will use them to solve its Grand Challenge research problems. These are fundamental problems whose solutions require significant increases in computational power and are critical to meeting national needs.

NASA's Grand Challenges include:

Improving the design and simulation of advanced aerospace vehicles;

Enabling people at remote locations to communicate more effectively and share information;

Increasing scientists' abilities to model the Earth's climate and forecast global environmental trends; and

Improving the capabilities of advanced spacecraft to explore the Earth and solar system.

NASA has initiated a program component to broaden the reach of the HPCC Program and begin the development of a National Information Infrastructure by supporting research and development in education, digital library technology, and access to Earth and space science data. The FY 1997 NASA HPCC Program has been organized into four vertically integrated computing projects:

The Computational Aerosciences (CAS) project;

The Earth and Space Sciences (ESS) project;

The Remote Exploration and Experimentation (REE) project; and

The Information Infrastructure Technology and Applications (IITA) component.

The REE project, inactive from FY 1993 through FY 1995, resumed activities in FY 1996, beginning with program definition and planning. This project seeks to provide scalable space borne computing to enable affordable and frequent missions involving powerful micro-instruments on sophistication miniature spacecraft to meet the exciting and challenging space science needs of the next century. The goal of REE is to demonstrate high performance computing capabilities for space borne applications afforded by a scalable family of low-cost, low power flight computers.

In cooperation with other participating Federal agencies, NASA's program involves deployment of experimental, scalable supercomputer capabilities essential for computational design of integrated aerospace vehicle systems and for predicting long-term global changes.

NASA conducts a series of workshops that address Federal HPCC Program needs in software technologies and algorithms, computational aerosciences, and supercomputing testbeds. NASA is responsible for implementing the National HPCC Software Exchange to foster software sharing and reuse across the Federal HPCC Program. NASA also is acquiring hardware for computational science testbeds and developing software tools to enhance productivity, including load balancing tools, run time optimizers, monitors, parallelization tools as well as data management and visualization tools.

The agency's NREN activity provides high-speed network connections among NASA, industry, and academic researchers.

NASA's Basic Research and Human Resources component encourages research into the underlying theory and concepts of high performance computing and will foster research in high performance computing at NASA centers, research institutes and universities.

The IITA component consists of critical information technologies and the application of these technologies to "National Challenges," problems where the application of HPCC technology can provide huge benefits to all Americans. 


\section{National Aeronautics and Space Administration}

$\overrightarrow{\vec{N}}$

\begin{tabular}{|c|c|c|c|c|c|c|c|c|c|c|c|}
\hline \multirow[b]{2}{*}{ Program Activity } & \multirow[b]{2}{*}{ Budget Account Code } & \multirow{2}{*}{\begin{tabular}{|} 
Partner/User \\
Agencies
\end{tabular}} & \multicolumn{4}{|c|}{ Budget $(\mathrm{BA}, \$ \mathrm{M})$} & \multicolumn{5}{|c|}{ HPCC PCAs by 1997 Pres. Request } \\
\hline & & & $\begin{array}{l}\text { FY 95 } \\
\text { Actual }\end{array}$ & $\begin{array}{l}\text { FY } 96 \\
\text { Pres. }\end{array}$ & $\begin{array}{c}\text { FY } 96 \\
\text { Est. }\end{array}$ & $\begin{array}{l}\text { FY } 97 \\
\text { Rast. }\end{array}$ & HECC & LSN & HCS & HuCs & ETHR \\
\hline Testbeds & \begin{tabular}{|c|}
$509-10,509-20,509-30,234,5$ \\
36
\end{tabular} & ARPA, NSF, & 25.12 & 14.81 & 12.91 & 15.39 & 15.39 & & & & \\
\hline Grand Challenge Support & \begin{tabular}{|c|}
$509-10,-20,-30,505,535-538$ \\
212232 \\
233306656 \\
\end{tabular} & $\begin{array}{l}\text { NSF, DOE, } \\
\text { NOAA EPA }\end{array}$ & 59.00 & 59.84 & 59.84 & 57.75 & 56.75 & & 1.00 & & \\
\hline Systems Software & $\begin{array}{|lll|}509-10 & 509-20 & 509-30 \\
\end{array}$ & $\begin{array}{l}\text { EPA, NOAA, } \\
\text { NSA NUH }\end{array}$ & 8.04 & 10.02 & 10.02 & 10.96 & 7.56 & & & 3.40 & \\
\hline NREN & $509-10,-20,428.538$ & DOE, DARPA, & 14.32 & 20.85 & 20.85 & 15.30 & & 15.30 & & & \\
\hline BRHR & $509-10,-20,233$ & $\begin{array}{l}\text { NSF, NIH, } \\
\text { NOAA FPA }\end{array}$ & 3.59 & 3.98 & 3.98 & 3.20 & & & & & 3.20 \\
\hline Information Infrastructure Technology & $509-40$ & $\begin{array}{l}\text { ARPA, NSF, } \\
\text { DOE NUH ED }\end{array}$ & 7.20 & 8.80 & 8.80 & 3.50 & 3.50 & & & & \\
\hline Information Infrastructure Applications & $509-40$ & NOAA, EPA, ED & 14.13 & 18.00 & 10.20 & 4.00 & 4.00 & & & & \\
\hline & & Totals: & 131.40 & 136.30 & 126.60 & 110.10 & 87.20 & 15.30 & 1.00 & 3.40 & 3.20 \\
\hline
\end{tabular}


National Aeronautics and Space Administration

\begin{tabular}{|c|c|c|c|c|c|c|c|c|c|c|c|c|c|c|c|}
\hline \multirow{2}{*}{\multicolumn{2}{|c|}{ Program Activity }} & \multicolumn{4}{|c|}{ Budget $(\mathrm{BA}, \$ \mathrm{M})$} & \multicolumn{5}{|c|}{ HPCC PCAs by 1997 Pres. Request } & \multicolumn{5}{|c|}{ HPCC PCAs by 1996 Estimated } \\
\hline & & $\begin{array}{l}\text { FY 95 } \\
\text { Actual }\end{array}$ & $\begin{array}{l}\text { FY } 96 \\
\text { Pres. }\end{array}$ & $\begin{array}{l}\text { FY } 96 \\
\text { Est. }\end{array}$ & $\begin{array}{l}\text { FY } 97 \\
\text { Rast. }\end{array}$ & HECC & LSN & HCS & HuCS & ETHR & HECC & LSN & HCS & HuCS & ETHR \\
\hline Testbeds & & 25.12 & 14.81 & 12.91 & 15.39 & 15.39 & & & & & 12.91 & & & & \\
\hline Grand Challenge Support & & 59.00 & 59.84 & 59.84 & 57.75 & 56.75 & & 1.00 & & & 52.62 & 6.60 & & & 0.62 \\
\hline Systems Software & & 8.04 & 10.02 & 10.02 & 10.96 & 7.56 & & & 3.40 & & 10.02 & & & & \\
\hline NREN & & 14.32 & 20.85 & 20.85 & 15.30 & & 15.30 & & & & & 20.85 & & & \\
\hline BRHR & & 3.59 & 3.98 & 3.98 & 3.20 & & & & & 3.20 & & & & & 3.98 \\
\hline Information Infrastructure Technology & & 7.20 & 8.80 & 8.80 & 3.50 & 3.50 & & & & & & & & & 8.80 \\
\hline Information Infrastructure Applications & & 14.13 & 18.00 & 10.20 & 4.00 & 4.00 & & & & & & & & & 10.20 \\
\hline & Totals: & 131.40 & 136.30 & 126.60 & 110.10 & 87.20 & 15.30 & 1.00 & 3.40 & 3.20 & 75.55 & 27.45 & & & 23.60 \\
\hline
\end{tabular}




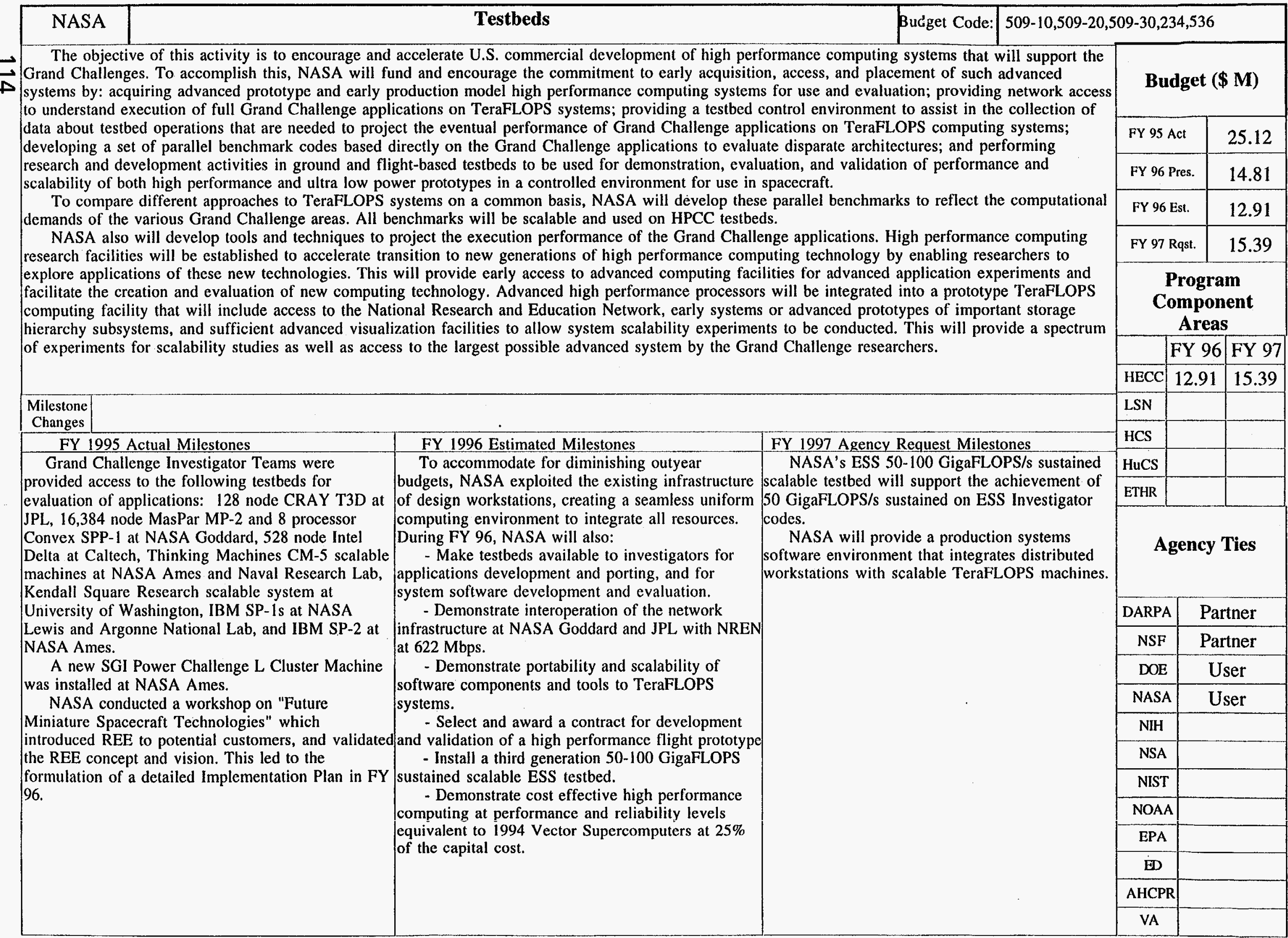


This research area develops, enhances, and evaluates techniques for the multidisciplinary modeling and simulation required by Grand Challenge problems. The Computational Aeroscience (CAS) research will focus on understanding the high performance computing environment and how it can be used to solve a range of problems of importance in aerospace engineering at a cost that represents the value, flexibility, and short cycle time required by the aerospace community. This will be accomplished with the aid and focus provided by several representative aerospace design problems related to nationally important programs. These are: a High Speed Civil Transport (HSCT); an Advanced Subsonics Civil Transport (ASCT); High-Performance Aircraft (HPA); and Rotorcraft.

Earth and Space Science (ESS) research will cover two critical scientific areas: the coupling of advanced discipline models into scalable global simulations providing realistic global change understanding; and the integration of models and analysis algorithms for processing, analyzing and understanding the enormous volumes of data expected from scientific missions. Research will focus on: large scale structure and galaxy formation; cosmology and accretion astrophysics; convective turbulence and mixing in astrophysics; solar activity and heliospheric dynamics; Earth system models; four-dimensional data assimilation; climate models, and knowledge discovery in geophysical databases and satellite data.

Collaborative groups including discipline scientists, software and systems engineers, professional software developers and algorithm designers are supported by shared computational and experimental facilities. Technical accomplishments will include development of application-specific codes for innovative high- performance computing systems, design and analysis of algorithms, and architecture and performance assessment of specific applications. NASA will evaluate the early research products and make the results available to system vendors as quickly as possible. Results in design and theory of algorithms are as important to breaking down computational scaling barriers as are performance improvements in computing hardware. Algorithms for common techniques such as multidimensional FFTs, Fast Poisson solvers, multigrid methods, Reimann solvers, sparse matrix methods, singular value decomposition, matrix factorization methods, and spectral methods are being re-implemented on a variety of architectures in order to understand how architecture affects efficiency and algorithm design.

\section{Milestone}

Changes

\section{FY 1995 Actual Milestones}

Developed multidisciplinary Earth and space science applications on 10-50 GigaFLOPS testbed, including: Cosmology and accretion astrophysics, large scale structure and galaxy formation,

convective turbulence and mixing in astrophysics, solar activity and heliospheric dynamics, atmosphere/ocean dynamics and tracers chemistry. The Earth and Space Science Team evaluated testbed architectures, running their scientific problems, for usability and efficiency.

Developed advanced subsonic civil transport analysis/optimization code and perform medium fidelity high speed civil transport modeling.

Enhanced the ability of aircraft manufacturers to quickly analyze different design options and accelerate the prototyping process, thereby reducing design cycle costs and producing vehicles with improved performance.

Demonstrated 32 GigaFLOPS sustained

performance on 144 nodes of the IBM SP-2 at NASA Ames in support of FY96 milestone to demonstrate $\rightarrow$ multidisciplinary aeroscience applications on 10-50 $\overrightarrow{\mathrm{v}}$ GigaFLOPS testbeds.

\section{FY 1996 Estimated Milestones}

Demonstrate multidisciplinary aeroscience and

Earth and Space Science applications on 10-50 GigaFLOPS testbeds.

Demonstrate end-to-end reductions in cost and time to solution for aerospace design applications on heterogeneous systems. The approach was to make a CAS testbed of heterogeneous workstations and high speed local networking openly accessible to software activities similar to existing HPCC work on TeraFLOPS systems. Design applications, oriented around the propulsion industry needs, guided the system software.

Select and award Round-2 ESS Investigators.

\section{FY 1997 Agency Request Milestones}

Demonstrate multidisciplinary Earth and Space Science applications sustaining 50-100 GigaFLOPS.

Complete TeraFLOPS scalable ESS and CAS arallel Benchmarks.

\section{on}

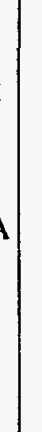

\begin{tabular}{|c|c|c|}
\hline \multicolumn{3}{|c|}{ Budget (\$ M) } \\
\hline \multicolumn{2}{|c|}{ FY 95 Act } & 59.00 \\
\hline \multicolumn{2}{|c|}{ FY 96 Pres. } & 59.84 \\
\hline \multicolumn{2}{|c|}{ FY 96 Est. } & 59.84 \\
\hline \multicolumn{2}{|c|}{ FY 97 Rqst. } & 57.75 \\
\hline \multicolumn{3}{|c|}{$\begin{array}{c}\text { Program } \\
\text { Component } \\
\text { Areas }\end{array}$} \\
\hline \multicolumn{2}{|c|}{ FY 96 } & FY 97 \\
\hline HECC & 52.62 & 56.75 \\
\hline LSN & 6.60 & \\
\hline $\mathrm{HCS}$ & & 1.00 \\
\hline \multicolumn{3}{|l|}{ HuCs } \\
\hline ETHR & 0.62 & \\
\hline \multicolumn{3}{|c|}{ Agency Ties } \\
\hline \multicolumn{3}{|l|}{ DARPA } \\
\hline NSF & \multicolumn{2}{|c|}{ Partner } \\
\hline DOE & \multicolumn{2}{|c|}{ Partner } \\
\hline NASA & \multicolumn{2}{|c|}{ User } \\
\hline \multicolumn{3}{|l|}{$\mathrm{NIH}$} \\
\hline \multicolumn{3}{|l|}{ NSA } \\
\hline \multicolumn{3}{|l|}{ NIST } \\
\hline NOAA & \multicolumn{2}{|c|}{ User } \\
\hline EPA & \multicolumn{2}{|c|}{ User } \\
\hline \multicolumn{3}{|l|}{$\mathrm{ED}$} \\
\hline \multicolumn{3}{|l|}{ AHCPR } \\
\hline VA & & \\
\hline
\end{tabular}

December 20, 1996 


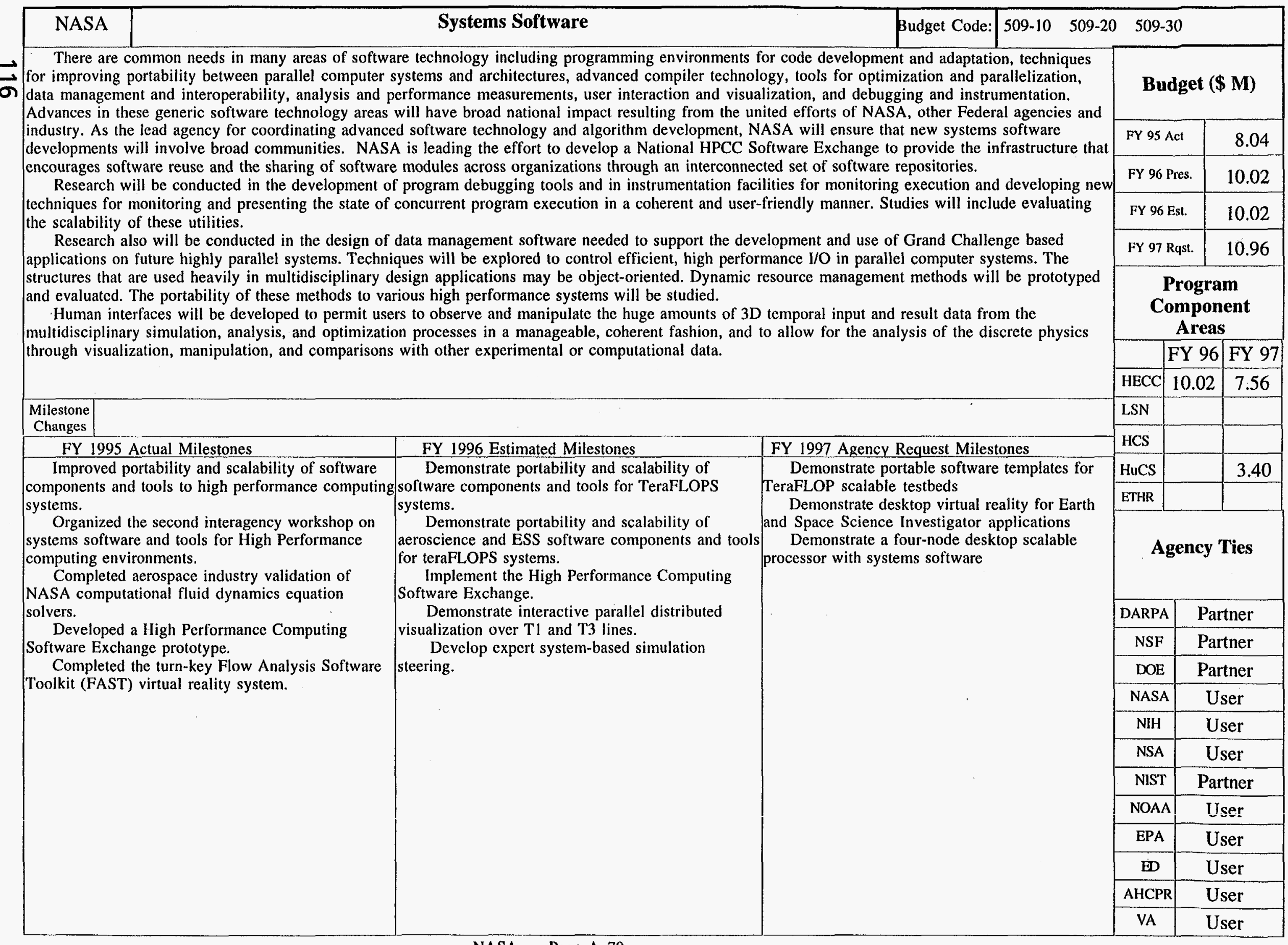


The National Research and Education Network (NREN) effort will establish standards and provide working models for commercial communications infrastructure deployment. NASA's role is to deploy the advanced communications required by the Grand Challenge investigators in a manner that satisfies the immediate needs of researchers while simultaneously guiding commercial infrastructure development for the nation NASA works with NSF, DOE, ARPA, and other agencies to enhance the national network infrastructure by coordinating the development and implementation of enhanced network technologies and services: integrated voice, video, and computer data transmission; network management and operations tools; protocol standards; routers and switches; security management; emerging high-performance user services including provision of advanced network services for multi-media communications; and K-12 distance learning and outreach applications.

NASA has deployed $45 \mathrm{Mb} / \mathrm{s}$ network services between five NASA centers and the NSFNET, significantly improving communications between remote investigators and NASA. An acquisition for advanced telecommunications services on an early availability basis will bring telecommunication and computational standards together to provide a low-cost computer network infrastructure over vendor facilities ultimately targeted at commercial availability This will take advantage of the latest telecommunications technologies, such as Asynchronous Transfer Mode (ATM) over Synchronous Optical Network Transmission (SONET) services, initially at $45 \mathrm{Mb} / \mathrm{s}$ among five NASA centers. Research collaboration with the DoD's Application Technology

Demonstration Network (ATDnet) supports interoperability between independently managed NREN networks that are based on ATM technology supplied by multiple vendors.

NASA also is working with other Federal HPCC agencies to extend the reach of NASA's data resources and computing capabilities to K-12 schools, libraries, and teacher resource centers. Further development of digital libraries and other networking infrastructure technologies under the IITA component will promote remote access to NASA HPCC resources, enabling schools and resource centers to collaborate directly with each other over networks on distributed NASA education projects. In order to incorporate satellite-based communications technology into the terrestrial gigabit highways of the future, two experiments are planned for coupling the NREN effort with the NASA's Advanced Communications Technology Satellite (ACTS).

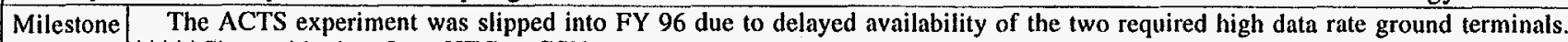
Changes $* * * * *$ Changed budget from HEC to GSN

\section{FY 1995 Actual Milestones}

Completed T-3 (45 Mb/s) Level 3 HPCC interconnects of five NASA centers -- Ames Research Center (ARC), Goddard Space Flight Center (GSFC), Langley Research Center (LaRC), Lewis Research Center (LeRC) and the Jet Propulsion Laboratory (JPL) -- with switched packet network to serve research communities.

Demonstrated satellite-based gigabit applications in using the Advanced Communications Technology Satellite (ACTS) and ground terminals.

Interconnected five NASA research centers. using $155 \mathrm{Mb} / \mathrm{s}$ high speed communication: Ames Research Center (ARC), Goddard Space Flight Center (GSFC), Langley Research Center (LaRC), Lewis Research Center (LeRC) and the Jet Propulsion Laboratory (JPL).
FY 1996 Estimated Milestones

Complete ACTS experiments "High Data ACTS Experiments for Performing Global Science: Keck Telescope and Global Climate Model".

Operate NREN in support of ESS science investigators and aerospace industrial research partners.

Demonstrate interoperability between independently managed NREN networks that are based on ATM technology supplied by multiple vendors. This capability is critical to NASA in order to meet growing internal requirements while maintaining compatibility with the evolving nationa network infrastructure that includes other Federal Agency networks as well as the private sector networks that will comprise the NII.

\section{FY 1997 Agency Request Milestones} Interconnect ESS and CAS testbeds at 622 Megabits/s.

Demonstrate network technologies needed for network management, cost accounting, security, and support of high performance applications in the NREN and NII.

\section{Budget (\$ M)}

FY 95 Act

FY 96 Pres.

FY 96 Est.

FY 97 Rqst 15.30

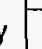

Program

Component

Areas

FY 96 FY 97

\begin{tabular}{|c|c|c|}
\hline HECC & & \\
\hline LSN & 20.85 & 15.30 \\
\hline HCS & & \\
\hline HUCS & & \\
\hline ETHR & & \\
\hline
\end{tabular}

Agency Ties

\begin{tabular}{|c|c|}
\hline DARPA & Partner \\
\hline NSF & Partner \\
\hline DOE & Partner \\
\hline NASA & User \\
\hline NIH & \\
\hline NSA & \\
\hline NIST & \\
\hline NOAA & User \\
\hline EPA & User \\
\hline ED & User \\
\hline AHCPR & \\
\hline VA & \\
\hline
\end{tabular}

December 20, 1996 


\begin{tabular}{|c|c|c|c|}
\hline NASA & BRHR & Budget Code: & 509-10,-20,233 \\
\hline & Effective integration of new high performance computing technology into the U.S. mainstream will require a sustained research effort across the spectrum \\
\hline of computing technology. Areas to be included are: computer architectures; fundamental algorithms; computational complexity; networked and distributed
\end{tabular}

$\infty$ computation; numerical analysis, and application specific algorithms.

NASA supports research institutes and centers of excellence engaged in computer science and computational science under the basic research and human resources component. These include: the Illinois Computer Laboratory for Aerospace Systems and Software (ICLASS) at the University of Illinois; the Research Institute for Advanced Computer Science (RIACS) at Ames Research Center; the Institute for Computer Applications in Science and Engineering (ICASE) at Langley Research Center; and the Center of Excellence for Space Data and Information Sciences (CESDIS) at Goddard Space Flight Center. In addition, NASA funds small university grants on HPCC topics at the individual principal investigator level.

The NASA HPCC program also focuses on the development of the next generations of computer and computational scientists. The resource potential to be developed will be concentrated at the graduate and postdoctoral level, but will extend to the baccalaureate and junior professor degree levels to a lesser extent. NASA's HPCC program will address the need to attract more talented scientists and engineers to computer and computational science in multiple ways by:

- Expanding ongoing NASA programs that support pre-baccalaureate and master's level training and research programs (e.g., ICLASS);

- Expanding doctoral degree research opportunities in High-Performance Computing (e.g., CESDIS, ICLASS);

- Expanding the ongoing NASA programs that support postdoctoral research and new professors (e. g., CESDIS, ICASE, RIACS, ICOMP).

In addition, new mechanisms for supporting students and new faculty interested in applying HPCC technology on NASA's applications have been initiated. This includes funding students directly at their institutions, provided they have an advisor interested in NASA applications, and expanding on the NASA Graduate Student Researchers Program at NASA centers and expanding support for National Research Council Resident Research Associates.

\section{Milestone}

Changes

FY 1995 Actual Milestones

Provided annual graduate and postdoctoral suppo

for high performance computing research.

Hosted fifth annual summer school in High

Performance Computational Physics.

\section{FY 1996 Estimated Milestones}

Provide annual graduate and postdoctoral suppor

for high performance computing research.

Host sixth annual summer school in High

Performance Computational Physics.

Transfer results of scalable $\mathrm{I} / \mathrm{O}$ research to commercial sector.

Publish Final Report of Round-1 Basic Compute

Science Research in key ESS technical areas.

Select and make Round-2 investigator awards

under ESS Basic Computer Science Research

Program
FY 1997 Agency Request Milestones

Provide annual graduate and postdoctoral

support for high performance computing research.

Host seventh annual summer school in High

Performance Computational Physics.
Budget (\$ M)

\begin{tabular}{|c|c|}
\hline FY 95 Act & 3.59 \\
\hline FY 96 Pres. & 3.98 \\
\hline FY 96 Est. & 3.98 \\
\hline FY 97 Rqst. & 3.20 \\
\hline
\end{tabular}

Program

Component

Areas

FY 96 FY 97

HECC

LSN

HCS

HuCs

ETHR

3.98

Agency Ties

\begin{tabular}{|c|c|}
\hline DARPA & \\
\hline NSF & Partner \\
\hline DOE & \\
\hline NASA & \\
\hline NIH & User \\
\hline NSA & \\
\hline NIST & \\
\hline NOAA & User \\
\hline EPA & User \\
\hline ED & \\
\hline AHCPR & \\
\hline VA & \\
\hline
\end{tabular}

December 20, 1996 


\begin{tabular}{|c|c|c|c|}
\hline NASA & Information Infrastructure Technology & Budget Code: & 509-40 \\
\hline NASA will promote the development and deployment of digital libraries within the National Information Infrastructure in partnership with other Feder
\end{tabular}

NASA will promote the development and deployment of digital libraries within the National Information Infrastructure in partnership with other Federal agencies. NASA's approach in pursuing this objective is based on unique NASA requirements and technology contributions, as well as on the more general advanced technology requirements of National Challenge applications.

NASA continues to participate in digital library technology research and development efforts begun under the NASA Cooperative Agreement Notice, "Public Use of Earth and Space Science Data Using the Internet", and the NSF/ARPA/NASA joint initiative "Research on Digital Libraries". A second

Cooperative Agreement Notice for research and development in digital library technology was planned to be issued in conjunction with the applications of Remote Sensing Data project. Successful cooperative efforts resulting from this solicitation will complement ongoing work.

NASA will work with aircraft and propulsion companies to facilitate the transfer of NASA HPCC-developed technology to users in major U.S. aerospace companies. This will be accomplished through projects to implement tools facilitating transmittal of proprietary data over networks. NASA will enable aeronautics community collaboration with NASA facilities without jeopardizing the confidentiality of competitive or proprietary data. This will be accomplished through an Authentication and Privacy project using systems analysis, integration and technology transfer to implement secure transmittal of remotely acquired aeronautics data.

\section{Milestone
Changes}

FY 1995 Actual Milestones

Initiated a digital library technology cooperative agreement with Loral Aerosys and Bowie State University.

Cosponsored a Digital Library workshop with NSF and ARPA to assess the state of the art and identify research directions.

Demonstrated prototype server for network progressive image transmission and compression.

Implemented "Space Update" kiosk at Houston Museum of Natural Sciences.

Identified authentication and privacy requirements of aeronautics industry and demonstrated use of commercial off the shelf (COTS) products to meet security requirements of the High Speed Research Project.

Identified Digital Library Technology

requirements of IITA remote sensing and NASA aeronautics projects.

Complete an IITA funded requirements analysis for an aeronautics Affordable Systems Optimization $\overrightarrow{0}$ Process (ASOP).

\section{FY 1996 Estimated Milestones \\ Identify additional digital library technologies needed to support remote sensing applications.} Develop and issue a second Cooperative Agreement Notice for Digital Library Technology and Remote Sensing Database Applications. Implement Earth Forum Kiosk module at Houston Museum of Natural Sciences.

Demonstrate Internet access over commercial cable television in 100 homes and schools in 3 cable districts in Maryland, Virginia, and Colorado. Demonstrate secure transmittal of proprietary simulations and wind tunnel test data between NASA installations and aeronautics industry partners.
FY 1997 Agency Request Milestones

Demonstrate integration of digital librar technologies with remote sensing demonstrations Release completed digital library software funded through IITA Cooperative Agreement Notice.
Budget (\$ M)

\begin{tabular}{|c|c|}
\hline FY 95 Act & 7.20 \\
\hline FY 96 Pres. & 8.80 \\
\hline FY 96 Est. & 8.80 \\
\hline FY 97 Rqst. & 3.50 \\
\hline
\end{tabular}

Program

Component

Areas

\begin{tabular}{|l|c|c|}
\hline & FY 96 & FY 97 \\
\hline HECC & & 3.50 \\
\hline LSN & & \\
\hline HCS & & \\
\hline HuCS & & \\
\hline ETHR & 8.80 & \\
\hline
\end{tabular}

Agency Ties

\begin{tabular}{|c|c|}
\hline DARPA & Partner \\
\hline NSF & Partner \\
\hline DOE & User \\
\hline NASA & User \\
\hline NIH & User \\
\hline NSA & \\
\hline NIST & User \\
\hline NOAA & User \\
\hline EPA & User \\
\hline ED & User \\
\hline AHCPR & \\
\hline VA & User \\
\hline
\end{tabular}

December 20, 1996 
$\rightarrow \quad$ NASA will develop and provide access to databases of remote sensing images and supportive software over the Internet. Such databases will be Naccessible to both public and private institutions, promoting the dissemination of taxpayer-funded Federal information. The information contained within O these databases can also be used by the educational and library communities to fulfill their needs and goals. The programmatic goal is to provide broad public access to remote sensing data to traditionally under-served communities. These data can be NASA and other federally funded data provided to the application communities. Providing the access to remote sensing data will promote gains in education, quality of life and economic growth.

NASA will build on its HPCC program, its aeronautics and space science research and engineering missions, and its existing education outreach infrastructure to facilitate the general development of the National Information Infrastructure to support mathematics, science, and engineering education in the K-12 levels. This will be done through both NASA field center 'outreach programs' and through open and competitive solicitations. In FY 95, NASA will issue a cooperative agreement notice open to public and private parties nationwide. The intent of this announcement will be address the National Challenge in "Education, Training, and Lifelong Learning" and will focus on doing this through the national aeronautics enterprise .

\section{Milestone \\ Changes}

FY 1995 Actual Milestones

New cooperative agreements were initiated with Childhood Project (Passport to Knowledge), Gulf of Maine Aquarium (Surfing the Net) and SENTAR

(Flood Management). Also, grants were initiated

with University of Michigan (Windows to the

Universe), University of California at Berkeley

(Science Information Infrastructure-SII), University

of Washington (The Puget Sound Project),

University of Wisconsin (Wisconsin Agriculture),

University of North Texas (Emergency and Crisis

Management-ECM) and Smithsonian Astrophysical Observatory (SAO).

Demonstrated public access and retrieval of

NASA databases by traditionally under-served communities.

"Connecting to the World."

Completed evaluation of IITA developed K-12 digital education material.

Provided a primary World Wide Web entry for the public to all the Digital Library Technology and Remote Sensing Database Applications projects through the Remote Sensing Public Access Center (RSPAC) in Fairmont, West Virginia.
FY 1996 Estimated Milestones

Award cooperative agreements and grants under a second "Public Use of Earth and Space Science Data over the Internet" Cooperative Agreement Notice.

Establish a World Wide Web server for GOES-8 data for use by funded projects and by the general public.

Demonstrate interim IITA remote sensing database applications over the Internet:

-Prototype weather broadcast videotape production: WRC-TV

- Demonstrate baseline maps and user interface: BADGER.

- Establish a World Wide Web server for GOES-8 data for use by IITA funded projects and by

\section{the general public.}

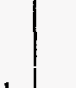

(

L

(2)

FY 1997 Agency Request Milestones Demonstrate integration of digital library

technologies with remote sensing demonstrations. Demonstrate completed Remote Sensing Database Applications over the Internet.

Demonstrate education products from K-14 Education Cooperative Agreement Notice. Issue a second K-14 Education Cooperative Agreement.

\section{Budget (\$ M)}

\begin{tabular}{|c|r|}
\hline FY 95 Act & 14.13 \\
\hline FY 96 Pres. & 18.00 \\
\hline FY 96 Est. & 10.20 \\
\hline FY 97 Rqst. & 4.00 \\
\hline
\end{tabular}

Program

Component

Areas

FY 96 FY 97

\begin{tabular}{|l|l|l|}
\hline HECC & & 4.00 \\
\hline LSN & & \\
\hline HCS & & \\
\hline HuCS & & \\
\hline ETHR & 10.20 & \\
\hline
\end{tabular}

Agency Ties

\begin{tabular}{|c|c|}
\hline DARPA & \\
\hline NŞF & \\
\hline DOE & Partner \\
\hline NASA & Partner \\
\hline NIH & \\
\hline NSA & \\
\hline NIST & \\
\hline NOAA & Partner \\
\hline EPA & Partner \\
\hline ED & User \\
\hline AHCPR & \\
\hline VA & \\
\hline
\end{tabular}

December 20, 1996 


\section{National Institutes of Health}

The National Institutes of Health (NIH) High Performance Computing and Communications (HPCC) programs are an integral part of the NIH biomedical research mission to develop basic knowledge for the diagnosis, treatment, understanding and prevention of human disease. The scientific activities include the analysis of biomolecular sequences and structures, the application of software tools for receptor-based drug design, the processing and visualization of biomedical images, and the modeling and simulation of living systems. The health care-related activities include the development of test bed networks linking hospitals, clinics, libraries, and medical schools, the development of computerized patient records and telemedicine technologies, and the creation of virtual environments to assist in medical diagnosis. The NIH HPCC programs make available to biomedical researchers the benefits of high performance computing and communication systems including advanced computing architectures and high speed network connections. The NIH pursues its HPCC goals through the funding of grants and contracts to support research conducted at universities and Research institutions throughout the Nation, as well as through research conducted at the NIH's intramural laboratories. These HPCC programs are administered by the National Library of Medicine (NLM), the National Center for Research Resources (NCRR), the Division of Computer Research and Technology (DCRT), the National Institute of General Medical Sciences (NIGMS), and the National Cancer Institute (NCI). 


\section{National Institutes of Health}

\begin{tabular}{|c|c|c|c|c|c|c|c|c|c|c|c|}
\hline \multirow[b]{2}{*}{ Program Activity } & \multirow[b]{2}{*}{ Budget Account Code } & \multirow{2}{*}{$\begin{array}{c}\text { Partner/User } \\
\text { Agencies }\end{array}$} & \multicolumn{4}{|c|}{ Budget (BA, $\$ M)$} & \multicolumn{5}{|c|}{ HPCC PCAs by 1997 Pres. Request } \\
\hline & & & $\begin{array}{l}\text { FY 95 } \\
\text { Actual } \\
\end{array}$ & $\begin{array}{c}\text { FY } 96 \\
\text { Pres. }\end{array}$ & $\begin{array}{c}\text { FY } 96 \\
\text { Est. }\end{array}$ & $\begin{array}{c}\text { FY } 97 \\
\text { Rgst. }\end{array}$ & HECC & LSN & HCS & HuCS & ETHR \\
\hline NLM Medical Connections Program & & & 0.61 & 0.62 & 0.82 & 1.22 & & 1.22 & & & \\
\hline NLM Electronic Imaging & & & 2.03 & 2.03 & 1.44 & 1.44 & & & & 1.44 & \\
\hline \multicolumn{12}{|l|}{ NLM Electronic Imaging (continued) } \\
\hline NLM Intelligent Agent DB searching & & & 5.35 & 5.37 & 8.37 & 8.57 & & 6.42 & & 2.15 & \\
\hline NLM HPCC Health Care Applications & & AHCPR, VA & 11.44 & 20.21 & 17.96 & 20.86 & & 3.33 & 3.55 & 13.98 & \\
\hline NCRR Biomolecular Computing & & $\overline{\mathrm{NSF}}$ & 7.60 & 6.60 & 6.60 & 7.30 & 6.30 & 0.20 & & 0.80 & \\
\hline NCRR Software Tools for Receptor-Based Drug Design & & NSF & 2.50 & 2.20 & 2.20 & 2.20 & 2.20 & & & & \\
\hline NCI Frederick Biomedical Supercomputing Center & & NSA, NIST & 5.40 & 5.40 & 5.90 & 5.90 & 3.60 & 1.39 & 0.14 & 0.28 & 0.49 \\
\hline $\begin{array}{l}\text { NCI High Speed Networking and Distributed } \\
\text { Conferencine }\end{array}$ & & & 2.00 & 1.70 & 1.34 & 1.31 & 0.20 & 0.72 & & 0.34 & 0.05 \\
\hline $\begin{array}{l}\mathrm{NCI} \text { High Perf. Comms for PDQ, CancerNet, and } \\
\text { Electranic Publishine }\end{array}$ & & & 0.60 & 0.60 & 0.60 & 0.60 & & 0.30 & & 0.30 & \\
\hline NIGMS & & NSF & & & & 0.50 & 0.50 & & & & \\
\hline & & Totals: & 67.35 & 77.91 & 79.74 & 86.21 & 23.40 & 22.90 & 4.94 & 29.59 & 5.38 \\
\hline
\end{tabular}




\section{National Institutes of Health}

\begin{tabular}{|c|c|c|c|c|c|c|c|c|c|c|c|c|c|c|}
\hline \multirow[b]{2}{*}{ Program Activity } & \multicolumn{4}{|c|}{ Budget $(\mathrm{BA}, \$ \mathrm{M})$} & \multicolumn{5}{|c|}{ HPCC PCAs by 1997 Pres. Request } & \multicolumn{5}{|c|}{ HPCC PCAs by 1996 Estimated } \\
\hline & $\begin{array}{l}\text { FY 95 } \\
\text { Actual } \\
\end{array}$ & $\begin{array}{l}\text { FY } 96 \\
\text { Pres }\end{array}$ & $\begin{array}{c}\text { FY } 96 \\
\text { Est }\end{array}$ & $\begin{array}{l}\text { FY } 97 \\
\text { Rqsit. }\end{array}$ & HECC & LSN & $\mathrm{HCS}$ & HuCS & ETHR & HECC & LSN & HCS & HuCS & ETHR \\
\hline NLM Medical Connections Program & 0.61 & 0.62 & 0.82 & 1.22 & & 1.22 & & & & & 0.82 & & & \\
\hline NLM Biotechnology Informatics & 4.32 & 4.35 & 5.67 & 6.17 & & 4.92 & 0.75 & 0.50 & & & 4.54 & 0.68 & 0.45 & \\
\hline NLM Electronic Imaging & 2.03 & 2.03 & 1.44 & 1.44 & & & & 1.44 & & & & & 1.44 & \\
\hline \multicolumn{15}{|l|}{ NLM Electronic Imaging (continued) } \\
\hline NLM HPCC Training Grants & 3.01 & 3.04 & 3.04 & 3.04 & & & & & 3.04 & & & & & 3.04 \\
\hline NLM IAIMS grants & 3.39 & 3.39 & 3.40 & 3.40 & & 2.00 & 0.50 & 0.90 & & & 2.00 & 0.50 & 0.90 & \\
\hline NLM Intelligent Agent DB searching & 5.35 & 5.37 & 8.37 & 8.57 & & 6.42 & & 2.15 & . & & 6.27 & & 2.10 & \\
\hline NLM HPCC Health Care Applications & 11.44 & 20.21 & 17.96 & 20.86 & & 3.33 & 3.55 & 13.98 & & & 2.87 & 3.06 & 12.03 & \\
\hline NCRR Biomolecular Computing & 7.60 & 6.60 & 6.60 & 7.30 & 6.30 & 0.20 & & 0.80 & & 5.80 & 0.20 & & 0.60 & \\
\hline NCRR Software Tools for Receptor-Based Drug Design & 2.50 & 2.20 & 2.20 & 2.20 & 2.20 & & & & & 2.20 & & & & \\
\hline NCRR Modeling/Simulation & 4.80 & 5.80 & 5.80 & 5.30 & 4.50 & 0.10 & & 0.70 & & 4.50 & 0.10 & & 1.20 & \\
\hline NCRR Virtual Reality/Environments & 4.00 & 4.20 & 4.20 & 7.70 & & & & 7.70 & & & & & 4.20 & \\
\hline NCRR HPCC Training & 1.40 & 3.50 & 3.50 & 1.80 & & & & & 1.80 & & & & & 3.50 \\
\hline $\begin{array}{l}\text { DCRT High Performance Biomedical Computing } \\
\text { Proeram }\end{array}$ & 8.90 & 8.90 & 8.90 & 8.90 & 6.10 & 2.30 & & 0.50 & & 6.10 & 2.30 & & 0.50 & \\
\hline NCI Frederick Biomedical Supercomputing Center & 5.40 & 5.40 & 5.90 & 5.90 & 3.60 & 1.39 & 0.14 & 0.28 & 0.49 & 3.60 & 1.39 & 0.14 & 0.28 & 0.49 \\
\hline $\begin{array}{l}\text { NCI High Speed Networking and Distributed } \\
\text { Confereuciug }\end{array}$ & 2.00 & 1.70 & 1.34 & 1.31 & 0.20 & 0.72 & & 0.34 & 0.05 & 0.20 & 0.72 & & 0.34 & 0.08 \\
\hline $\begin{array}{l}\mathrm{NCl} \text { High Perf. Comms for PDQ, CancerNet, and } \\
\text { Electronic_Ruhlishino }\end{array}$ & 0.60 & 0.60 & 0.60 & 0.60 & & 0.30 & & 0.30 & & & 0.30 & & 0.30 & \\
\hline NIGMS & & & & 0.50 & 0.50 & & & & & & & & & \\
\hline Totals: & 67.35 & 77.91 & 79.74 & 86.21 & 23.40 & 22.90 & 4.94 & 29.59 & 5.38 & 22.40 & 21.51 & 4.38 & 24.34 & 7.11 \\
\hline
\end{tabular}




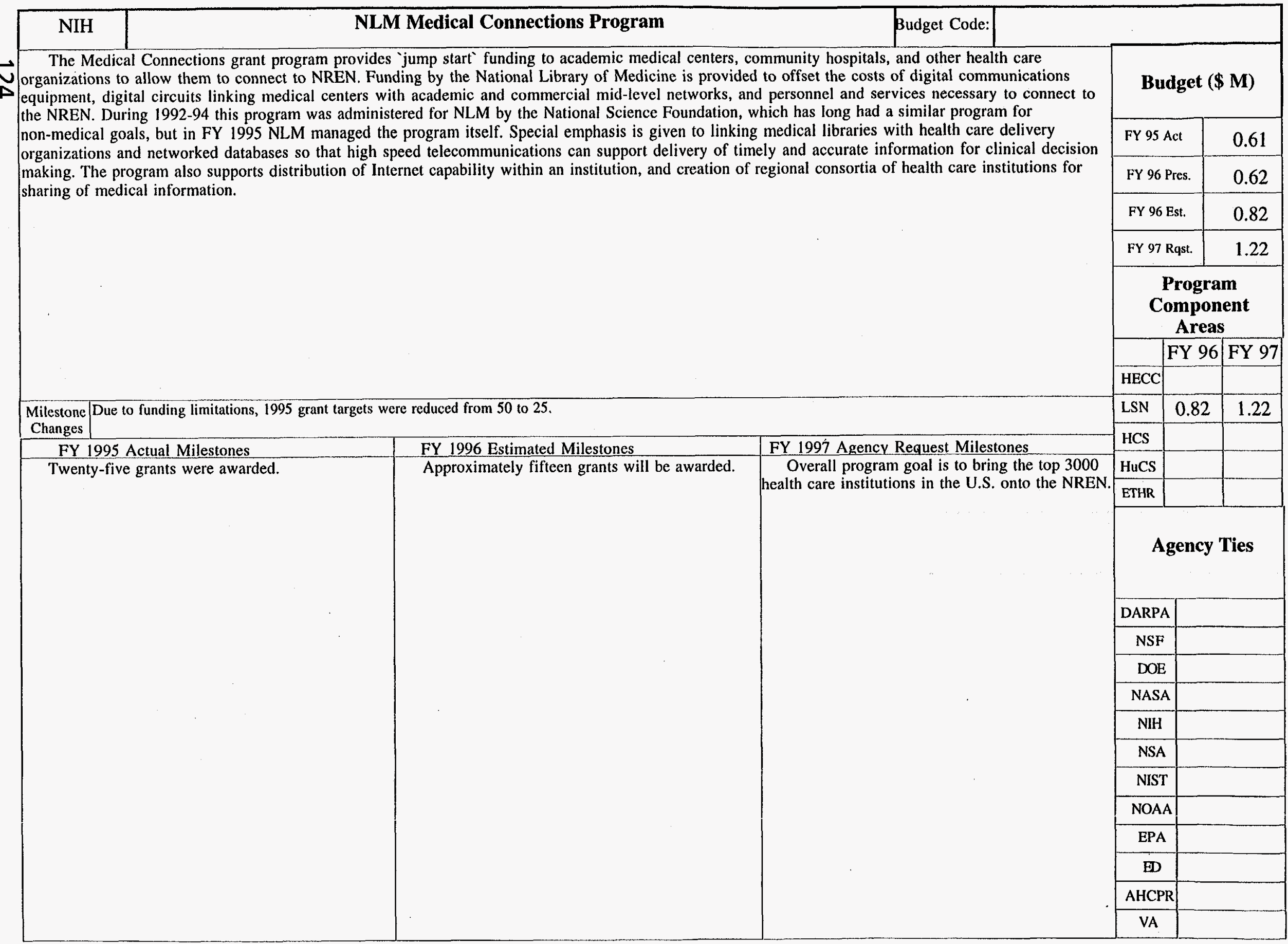




\begin{tabular}{|c|c|c|}
\hline NIH & NLM Biotechnology Informatics & Budget Code: \\
\hline
\end{tabular}

The NLM's National Center for Biotechnology Information (NCBI) has been given the legislative mandate to create automated systems for storing and analyzing the vast and growing volume of data related to molecular biology, biochemistry, and genetics. This information science is an essential component of genome research and protein engineering and drug design, and seeks to develop analytical and predictive methods to identify key molecular patterns associated with health and disease as represented in a large number of related data banks encoding DNA, RNA, proteins, and other biologically important molecules. NCBI is implementing an integrated database of molecular sequence and structure which contains key linkages to the scientific literature and to existing biological data banks; the database is available to researchers nationally via the Internet using special purpose server-client software as well as the World Wide Web. Research on efficient and expressive data representation techniques for molecular sequence objects is conducted within NCBI's Computational Biology Branch and has lead to the development of new sequence analysis and retrieval methods. Within this distributed database architecture, the Center builds and provides access to GenBank, the NIH DNA sequence databank which is a key data resource of the Human Genome Project. The Biotechnology Informatics program administered through NLM's Extramural Program also supports investigator-initiated research in computational biology via peer-reviewed grants.

\section{Milestone \\ Changes}

FY 1995 Actual Milestones

Tenfold increase in linkage of gene sequences to

related scientific literature. Linkage of sequence data pace of input to database, from the current rate of

to three-dimensional protein structure data.

Coordinated effort with Washington University

and Merck to add over 200,000 human cDNA

sequences to GenBank.

Completed implementation of network-based sequence submission software.

doubling every 20 months. High throughput from database in less than one year.

NCBI begins distribution of human genetic disease database produced at Johns Hopkins. Increased user and network demands for cDNA sequencing may is expected to double size sequence search and retrieval, now approaching 30,000 queries per day, will require upgrading server software and hardware.

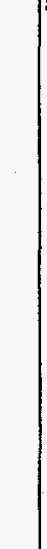

FY 1997 Agency Request Milestones

Increase in the number of funded genome

centers will increase sequence data output to

greater than 500,000 sequences per year.

Increased demand for retrieval services due to growth in Internet and the World Wide Web and the availability of complete genome from several organisms.

NCBI will continue participation with publishers of online journals in a WWW project linking the GenBank sequences and MEDLINE abstracts to the full text of scientific articles. Immensity and complexity of genomic data will drive development of tools for synthesizing and summarizing data into higher level interactive views.

\section{Budget ( $\$$ M)}

\begin{tabular}{|c|c|c|}
\hline \multicolumn{2}{|c|}{ FY 95 Act } & 4.32 \\
\hline \multicolumn{2}{|c|}{ FY 96 Pres. } & 4.35 \\
\hline \multicolumn{2}{|c|}{ FY 96 Est. } & 5.67 \\
\hline \multicolumn{2}{|c|}{ FY 97 Rqst. } & 6.17 \\
\hline \multicolumn{3}{|c|}{$\begin{array}{c}\text { Program } \\
\text { Component } \\
\text { Areas }\end{array}$} \\
\hline & FY 96 & FY 97 \\
\hline \multicolumn{3}{|l|}{ HECC } \\
\hline LSN & 4.54 & 4.92 \\
\hline HCS & 0.68 & 0.75 \\
\hline HuCs & 0.45 & 0.50 \\
\hline ETHR & & \\
\hline
\end{tabular}

\section{Agency Ties}

\begin{tabular}{|c|c|}
\hline DARPA & \\
\hline NSF & \\
\hline DOE & \\
\hline NASA & \\
\hline NIH & \\
\hline NSA & \\
\hline NIST & \\
\hline NOAA & \\
\hline EPA & \\
\hline ED & \\
\hline AHCPR & \\
\hline VA & \\
\hline
\end{tabular}

December 20, 1996 
Images are an important part of biomedical knowledge. New computer-based technologies are providing an unprecedented opportunity to supplement the $\vec{N}$ traditional two dimensional images of medicine and biology with dynamic, three-dimensional images that can be viewed, rotated, and reversibly dissected

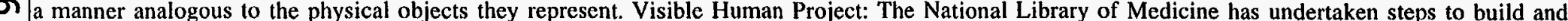
evaluate digital image libraries of anatomical structures of the human body. Full use and understanding of the biological structures depicted in such libraries will exploit the integration of advance computer and communications technologies, with medical imaging systems for computer tomography (CT), and magnetic resonance (MR)imaging. The combinations of these technologies with efficient algorithms to efficiently render anatomic data into photo realistic images which are easily manipulable by students, researchers, or health care providers will offer new tools for health education, research and clinical practice.

DPXNET Program: The NLM is the technical lead agency in a collaborative project in electronic imaging with the National Center for Health Statistics (NCHS) and the National Institute of Arthritis and Musculoskeletal and Skin Diseases (NIAMS). The NCHS conducts nationwide surveys and collects a broad range of medical, demographic and other health related data in addition to $x$-rays. This project has succeeded in digitizing and archiving about 17,000 cervical and lumbar spine x-ray films acquired as part of the second National Health and Nutrition Examination Survey (NHANES II) to create an electronic archive of this unique and nationally important collection. The third survey, NHANES III, has generated an additional 10,000 $x$-rays of the hands, wrists and knees.

Accomplishments include: the development of high performance imaging workstations for quality control and standardized readings; the development of an electronic store implemented by a 144-platter optical disk jukebox and a RAID system accessible over the Internet; the development of client/server software for general access to the images and collateral data over the Internet; initiating the design of an electronic radiologic atlas and training set for the cervical and lumbar spine; began the development of an integrated database with image and collateral data for general access over the Internet and begin the development of a general access workstation. (continued)

\section{Milestone}

Changes

\section{FY 1995 Actual Milestones}

The complete data sets for the Visible Human Male and Female were mounted and distributed both over the Internet, and as a published volume of $8 \mathrm{~mm}$ and $4 \mathrm{~mm}$ tapes.

Completed the scanning, quality control and archiving of 17,000 NHANES II spine $x$-ray images.

Developed, tested and demonstrated an extension of the electronic $x$-ray archive by integrating a RAID system with an optical disk jukebox.

Demonstrated a prototype standardized readings workstation in the lab, and conducted an evaluation of this workstation for determining optimum design parameters for user interface; using this evaluation, began the design of a portable standardized reading workstation for deployment on the Internet.

Began the design of an online electronic radiological atlas and training set for spine images, and selected image sets for these tools.

\section{FY 1996 Estimated Milestones}

A subset for the color data will be mapped with anatomical labels and graphical outlines to serve a data structure model for object identification of the full male and female image data sets; a

prototype image database will be designed to house $2 \mathrm{D}$ and $3 \mathrm{D}$ data sets

Continue research into automated image classification, and linkage of structural data with semantic labels.

Begin the design of a portable standardized readings workstations for deployment on the Internet; plan to begin readings of all cervical and lumbar spine images, and incorporate this data with the digital images and other NHANES II collateral data.

Complete general access client workstations for Internet access by universities, government

agencies, research institutions and a broad array of independent investigators; conduct beta tests of the general access workstations by selected

communities investigating osteoarthritis and other musculoskeletal diseases.

Begin capture, quality control and archiving of 10,000 NHANES III x-ray images of hands, wrists and knees.

(continued)

\section{FY 1997 Agency Request Milestones}

Implement the prototype for the complete mage data base.

Start full object identification of the Visible Human data sets.

Mount the prototype Visible Human Subset

Database on line for user beta testing.

Complete standardized readings of NHANES I and III images.

Expand general access to integrated database consisting of NHANES II and III collateral tex and images.

Expand general access workstations to access ther image/text databases.

Identify the compression technique and pecific parameters for maximum compression of mages consistent with required quality standards. Encode the multi socket transmission echnique for use in server/client systems.

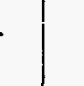

\section{Budget (\$ M)}

\begin{tabular}{|c|r|}
\hline FY 95 Act & 2.03 \\
\hline FY 96 Pres. & 2.03 \\
\hline FY 96 Est. & 1.44 \\
\hline FY 97 Rqst. & 1.44 \\
\hline
\end{tabular}

Program

Component

Areas

\begin{tabular}{|l|l|l|}
\hline & FY 96 & FY 97 \\
\hline HECC & & \\
\hline LSN & & \\
\hline HCS & & \\
\hline HuCS & 1.44 & 1.44 \\
\hline ETHR & & \\
\hline
\end{tabular}

Agency Ties

\begin{tabular}{|c|c|}
\hline DARPA & \\
\hline NSF & \\
\hline DOE & \\
\hline NASA & \\
\hline NIH & \\
\hline NSA & \\
\hline NIST & \\
\hline NOAA & \\
\hline EPA & \\
\hline ED & \\
\hline AHCPR & \\
\hline VA & \\
\hline
\end{tabular}

December 20, 1996 


\begin{tabular}{|c|c|c|c|}
\hline NIH & NLM Electronic Imaging (continued) & Budget Code: & \\
\hline $\begin{array}{c}\text { (continued) } \\
\text { ISTO Project: The larger size of the Visible Human image set and other medical images offer an enormous challenge to storage and transmission. The }\end{array}$
\end{tabular}

full set of Visible Human images would require a capacity of over $100 \mathrm{CD}$-ROMs, an impractical distribution option. The NLM therefore is investigating advanced compression and communication techniques to minimize the required storage capacity and maximize transmission speed over the Internet.

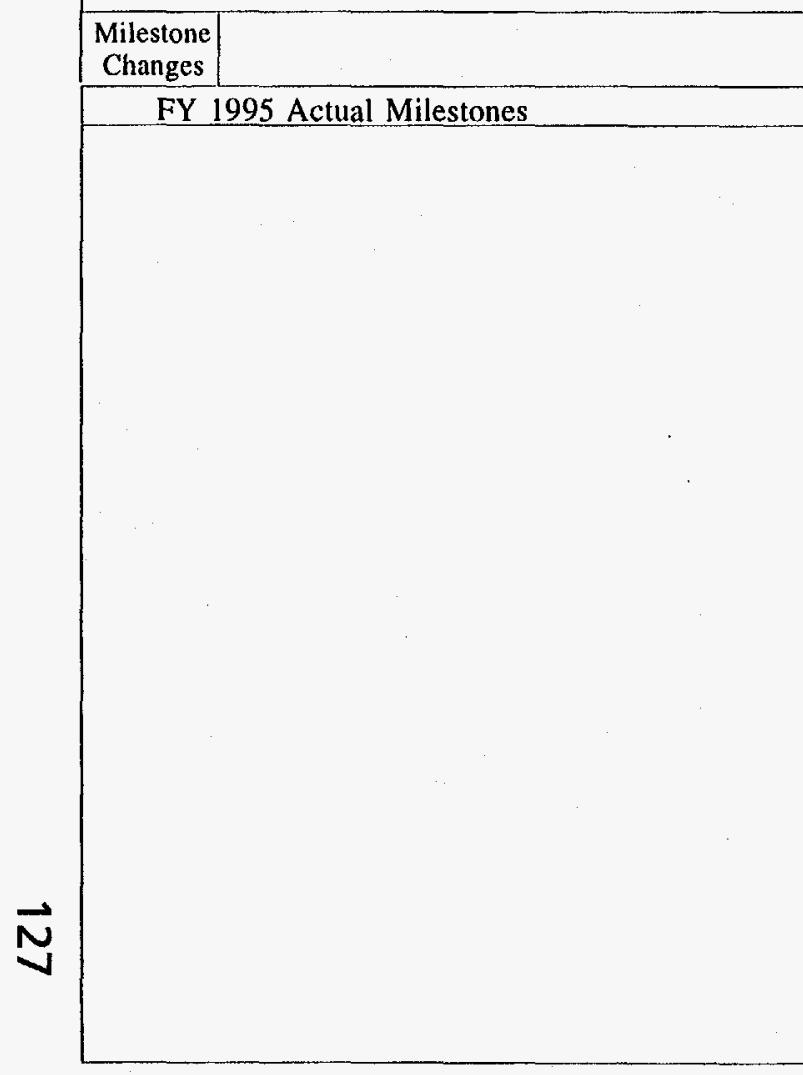

FY 1996 Estimated Milestones (continued)

Begin an effort to incorporate image

compression and high speed transmission techniques

to optimize the distribution of the Visible Human

and digital $x$-ray images. The multi socket

transmission technique algorithms will be tested on

image samples from both collections.

\section{FY 1997 Agency Request Milestones}

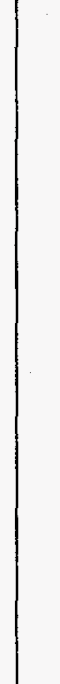

Budget (\$ M)

\begin{tabular}{|l|l|}
\hline FY 95 Act & \\
\hline FY 96 Pres. & \\
\hline FY 96 Est. & \\
\hline FY 97 Rqst. & \\
\hline
\end{tabular}

Program

Component

Areas

FY 96 FY 97

\begin{tabular}{|l|l|l|}
\hline HECC & & \\
\hline LSN & & \\
\hline HCS & & \\
\hline HUCS & & \\
\hline ETHR & & \\
\hline
\end{tabular}

Agency Ties

\begin{tabular}{|c|c|}
\hline DARPA & \\
\hline NSF & \\
\hline DOE & \\
\hline NASA & \\
\hline NIH & \\
\hline NSA & \\
\hline NIST & \\
\hline NOAA & \\
\hline EPA & \\
\hline ED & \\
\hline AHCPR & \\
\hline VA & \\
\hline
\end{tabular}

December 20, 1996 


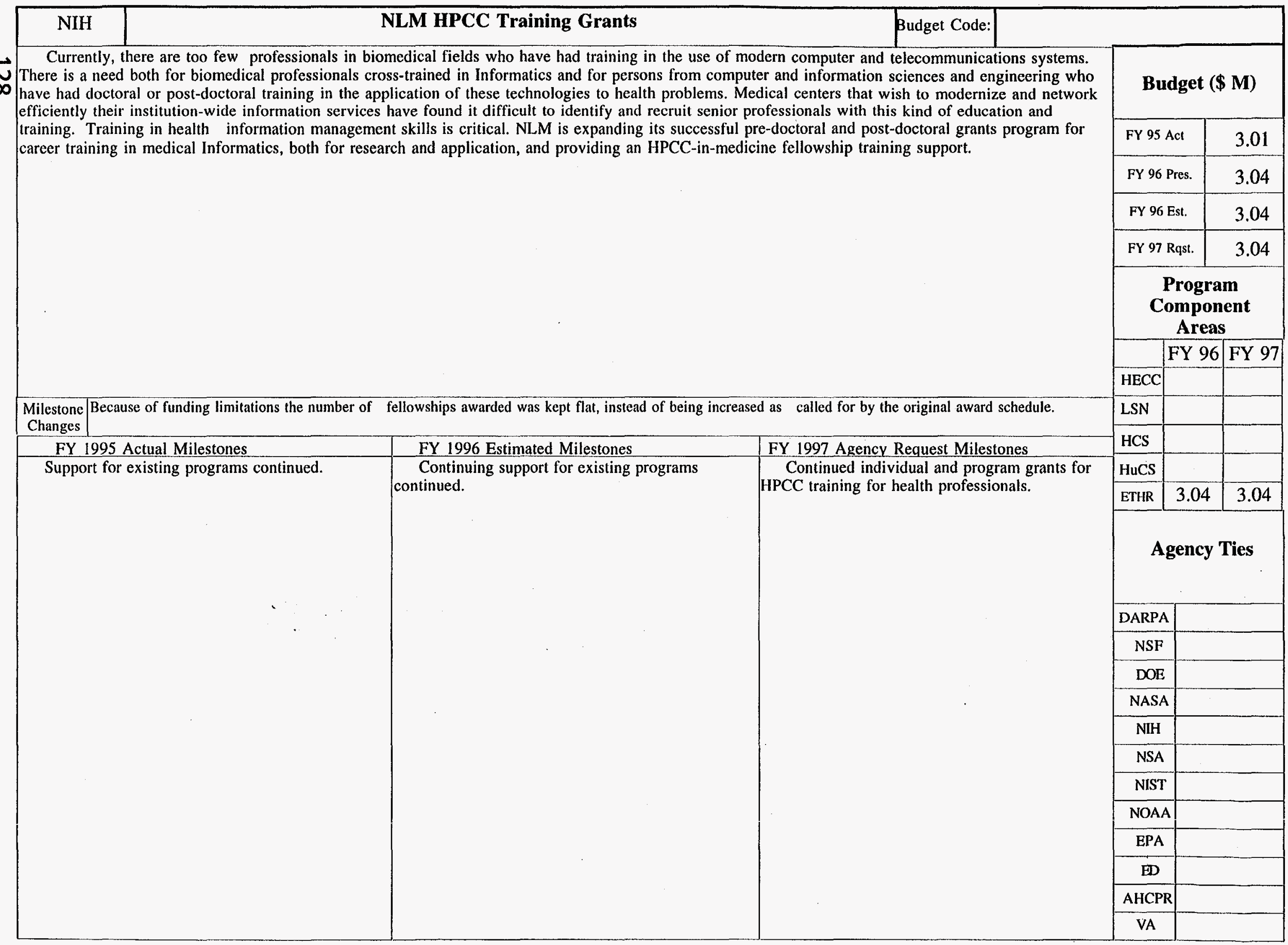


Academic Medical Centers are the backbone of the American biomedical research enterprise. These 120-plus institutions are comprised of health profession schools, their associated teaching and research hospitals, clinics and laboratories. Information related to patient care, research, education, and administration is the life blood of these complex centers; increasingly this information is in electronic form: databases of bibliographic and factual information, molecular databases, patient records, laboratory and clinical data. Currently, these electronic information sources (databases) are largely disconnected and isolated from one another, and communications among the various computerized systems in academic medical centers is primitive or non-existent. The focus of the IAIMS program, initiated in 1984, is the development of the technical and organizational infrastructure necessary to link and retrieve conceptually related information from many disparate sources within the medical center, and to link medical centers. The administrative, clinical, educational, and research databases should be able to communicate, and to appear as one database to the user. The goal of the program is the development, testing and implementation of generalizable systems of information flow management within university health science centers or major teaching hospitals. The expected outcomes of this program are greater research productivity, improved access to patient data for technology assessment and health outcomes research, and more efficient patient care leading to increased efficiency in the use of health care resources. The work is expected eventually to benefit al health delivery organization, including community hospitals and outpatient services.

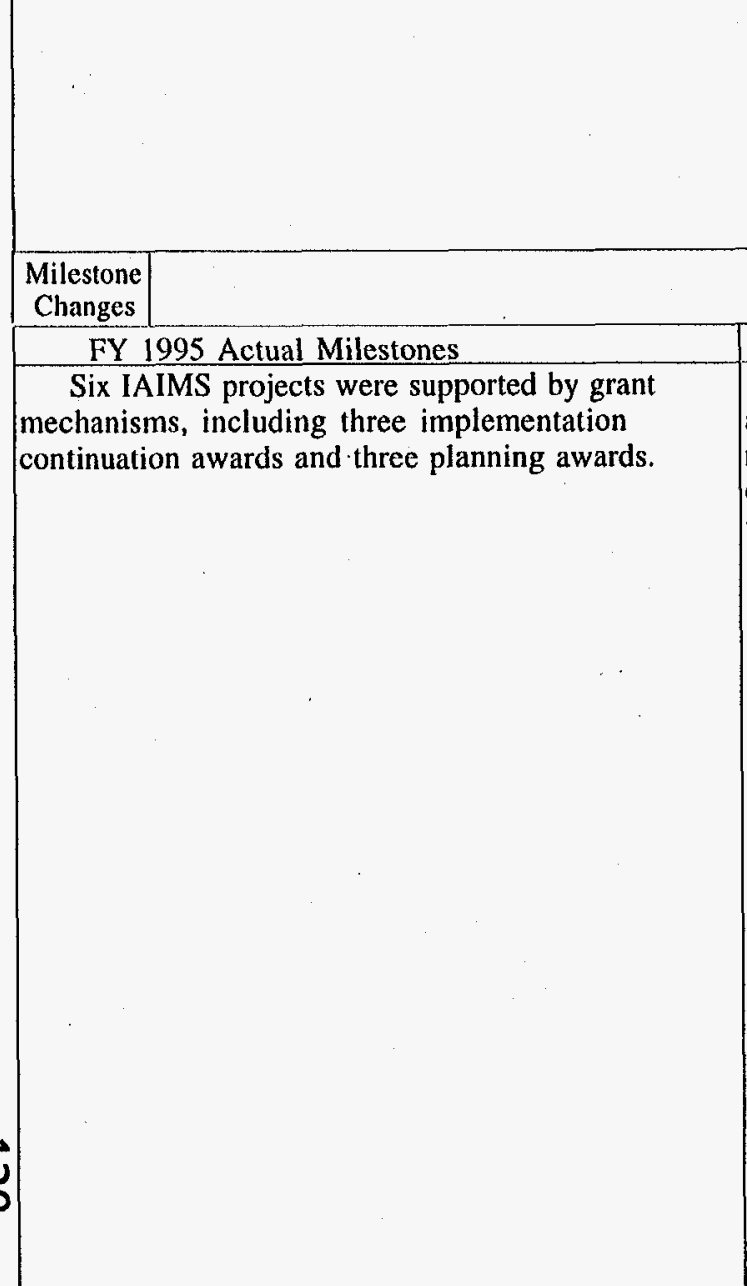

\section{FY 1996 Estimated Milestones}

In addition to continuation of active awards, approximately four new planning awards and two new implementation awards will be made; emphasis on sharing of information via the NREN will be a central focus of the program.

\section{FY 1997 Agency Request Milestones}

Continued progress toward integration of

academic information management by American medical centers.

\section{Budget (\$ M)}

\begin{tabular}{|c|c|c|}
\hline \multicolumn{2}{|c|}{ FY 95 Act } & 3.39 \\
\hline \multicolumn{2}{|c|}{ FY 96 Pres. } & 3.39 \\
\hline \multicolumn{2}{|c|}{ FY 96 Est. } & 3.40 \\
\hline \multicolumn{2}{|c|}{ FY 97 Rqst. } & 3.40 \\
\hline \multicolumn{3}{|c|}{$\begin{array}{c}\text { Program } \\
\text { Component } \\
\text { Areas }\end{array}$} \\
\hline & FY 96 & FY 97 \\
\hline \multicolumn{3}{|l|}{ HECC } \\
\hline LSN & 2.00 & 2.00 \\
\hline HCS & 0.50 & 0.50 \\
\hline HuCS & 0.90 & 0.90 \\
\hline ETHR & & \\
\hline
\end{tabular}

Agency Ties

\begin{tabular}{|c|c|}
\hline DARPA & \\
\hline NSF & \\
\hline DOE & \\
\hline NASA & \\
\hline NIH & \\
\hline NSA & \\
\hline NIST & \\
\hline NOAA & \\
\hline EPA & \\
\hline ED & \\
\hline AHCPR & \\
\hline VA & \\
\hline
\end{tabular}




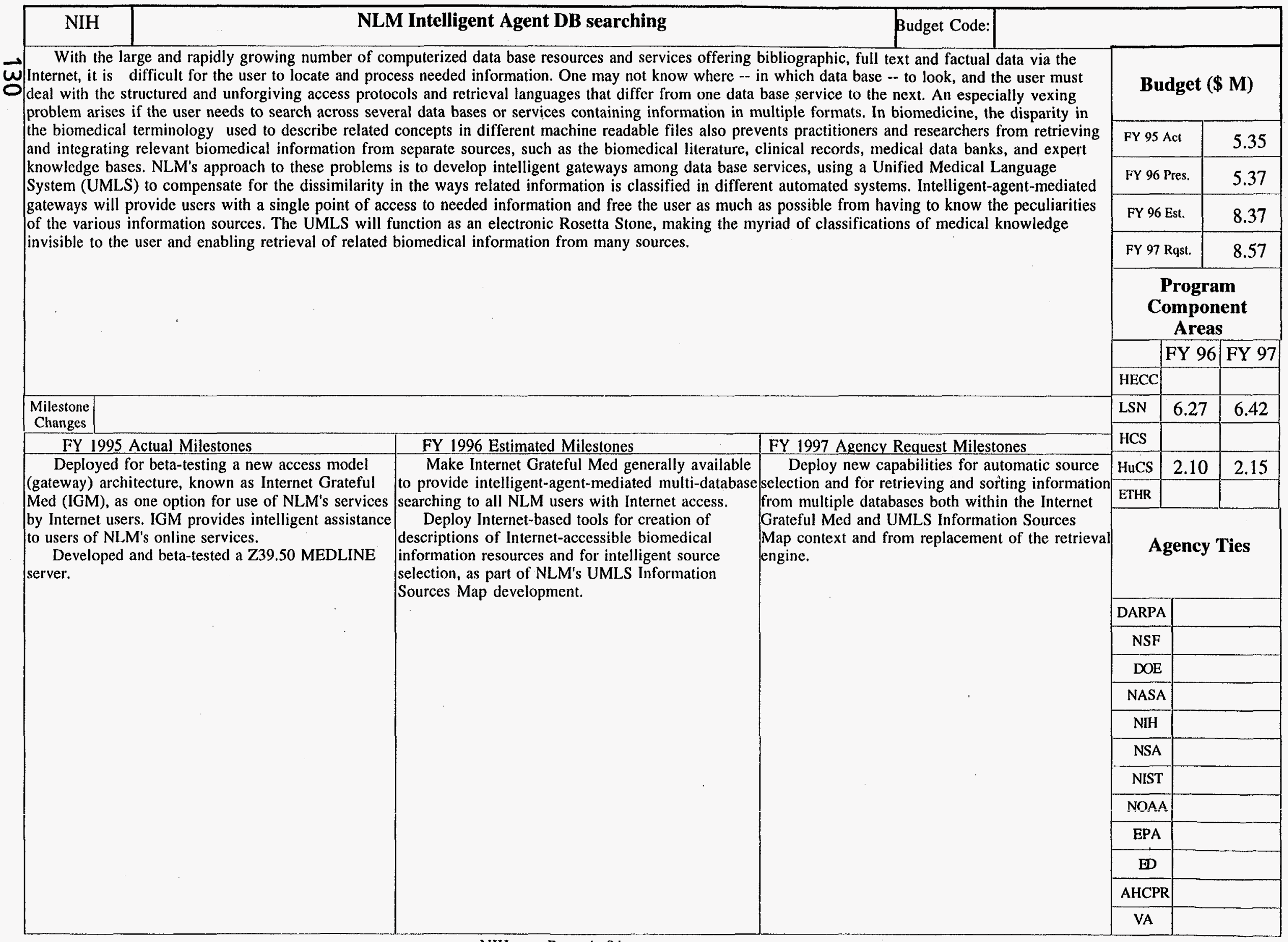




\begin{tabular}{|c|c|c|}
\hline NIH & NLM HPCC Health Care Applications & Budget Code: \\
\hline
\end{tabular}

Through a variety of mechanisms, the NLM is promoting the application of HPCC technologies to health care. The program supports research and development projects in each of the following IITA areas:

1. Test bed networks for linking hospitals, clinics, doctor's offices, medical schools, medical libraries, and universities to enable health care providers and researchers to share medical data and imagery;

2. Software and technology for visualizing the human anatomy and analyzing imagery from X-rays, CAT scans, PET scans, and other diagnostic tools;

3. Virtual reality technology for simulating operations and other medical procedures;

4. Telemedicine or collaborative technology to allow several health care providers in remote locations to provide real-time treatment to patients;

5. Database technology to provide health care providers with access to relevant medical information and literature; and

6. Database technology for storing, accessing, and transmitting patients' medical records while protecting the accuracy and privacy of those records.

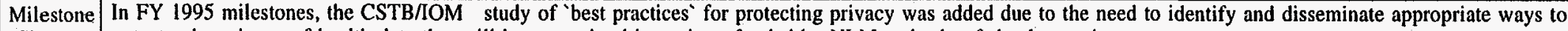
Changes protect the privacy of health data that will be transmitted in projects funded by NLM and other federal agencies.

FY 1995 Actual Milestones

BAA three-year awards and the initial cooperative agreements.

Funded 3 additional cooperative agreements. (2 NLM; 1 AHCPR)

Funded, in conjunction with the Health Care Financing Administration and the Department of

Veterans Affairs, a study by the Institute of Medicine

of the National Academy of Sciences to identify the range of criteria appropriate to the evaluation of telemedicine projects.

Funded, in conjunction with the NIH Clinical Center, a study by the Computer Sciences and Technology Board and the Institute of Medicine of the National Academy of Sciences of current 'best practices' and areas for research in protecting the privacy of electronic health care data transmitted over networks.

\section{Continue funding projects promoting the}

applications, with special emphasis on evaluation of application of HPCC technologies to health care, impact of telemedicine and testing of best practices the evaluation of telemedicine, and the testing of for protecting privacy of health data.

Continue funding of (welve HPCC BAA awards and $8(5.5 \mathrm{NLM} ; 25 \mathrm{AHCPR})$ c00perative agreements.$$
\text { heal }
$$
health data. There will also be a special emphas public health applications of the NII.
Budget (\$ M)

\begin{tabular}{|c|c|}
\hline FY 95 Act & 11.44 \\
\hline FY 96 Pres. & 20.21 \\
\hline FY 96 Est. & 17.96 \\
\hline FY 97 Rqst. & 20.86 \\
\hline
\end{tabular}

Program

Component

Areas

HECC

\begin{tabular}{|l|c|c|}
\hline LSN & 2.87 & 3.33 \\
\hline HCS & 3.06 & 3.55 \\
\hline HuCS & 12.03 & 13.98 \\
\hline ETHR & & \\
\hline
\end{tabular}

Agency Ties

\begin{tabular}{|c|c|}
\hline DARPA & \\
\hline NSF & \\
\hline DOE & \\
\hline NASA & \\
\hline NIH & \\
\hline NSA & \\
\hline NIST & \\
\hline NOAA & \\
\hline EPA & \\
\hline ED & \\
\hline AHCPR & Partner \\
\hline VA & Partner \\
\hline
\end{tabular}

December 20, 1996 
Biomolecular computing using high. performance computing involves extensive, often complex calculations to determine or predict:

1. The structure of biologically relevant macromolecules, e.g., proteins;

2. Their structural and functional changes due to interactions with other molecules or drugs;

3. How they are made in the cell and how they fold;

4. How they interact with water and biological membranes, and

5. Especially for drugs, the energetics of molecules going into solution.

Achieving meaningful results in a reasonable timeframe requires powerful computers and efficient software and algorithms. Hardware, including massively parallel processors, are available to several NCRR resource centers through a partnership with the NSF. Investigators at these resource centers develop the algorithms and software to address these important and difficult research problems.

Budget (\$ M)

\begin{tabular}{|c|c|c|}
\hline \multicolumn{2}{|c|}{ FY 95 Act } & 7.60 \\
\hline \multicolumn{2}{|c|}{ FY 96 Pres. } & 6.60 \\
\hline \multicolumn{2}{|c|}{ FY 96 Est. } & 6.60 \\
\hline \multicolumn{2}{|c|}{ FY 97 Rqst. } & 7.30 \\
\hline \multicolumn{3}{|c|}{$\begin{array}{c}\text { Program } \\
\text { Component } \\
\text { Areas }\end{array}$} \\
\hline & FY 96 & FY 97 \\
\hline HECC & 5.80 & 6.30 \\
\hline LSN & 0.20 & 0.20 \\
\hline \multicolumn{3}{|l|}{ HCS } \\
\hline HuCs & 0.60 & 0.80 \\
\hline THR & & \\
\hline
\end{tabular}

Fy 1997 Agency Request Milestones

Methodology to predict protein structure from

Changes
FY 1995 Actual Milestones

Research on methodology to determine protein

structure from sequence in conjunction with NMR

experiments further developed to become available

commercially. Improved methods to solve the

multiple-minima problem.

Completed study of a complex of phospholipase A2 with a membrane, involved in many disorders

such as toxic shock after injury, asthma, and arthritis.

Completed study of a related glucocorticoid

receptor and initiated a related study on a

computational technology, both hardware and V protein.

complexes of proteins and DNA in a water
environments. Support new investigator-initiated

in human cancers. Applied integration of graphics $\quad \begin{aligned} & \text { environments. Support } \\ & \text { research project grants. }\end{aligned}$

and molecular dyna

design using massively parallel processors. pructures at least five times faster than currently

possible. At least a partial solution of the protein

lding problem will have been achieved.

A complete study of DNA binding protein p53

Several resource centers operational to support quence and NMR data ready for

commercialization.

Improve methods for predicting final protein structure from its amino acid sequence.

Continued improvements in computational technology capable of addressing increasingly larger complexes of proteins and DNA in water environment.

Support new investigator-initiated research project grants.
Agency Ties

\begin{tabular}{|c|c|}
\hline DARPA & \\
\hline NSF & Partner \\
\hline DOE & \\
\hline NASA & \\
\hline NIH & \\
\hline NSA & \\
\hline NIST & \\
\hline NOAA & \\
\hline EPA & \\
\hline ED & \\
\hline AHCPR & \\
\hline VA & \\
\hline
\end{tabular}


The goal of this activity is to develop computational methodologies for use in the design of drugs. This endeavor includes the establishment of high performance computer-based environments that:

(1) accurately and efficiently estimate electrostatic forces in molecular and atomic interactions;

(2) effectively employ core computer technologies to calculate drug-protein binding energies from quantum mechanics, statistical mechanics and simulation techniques; and

(3) eventually attain dramatic improvements in performance of molecular dynamics programs to permit theoretical and experimental studies to be executed in similar time frames.

\section{Milestone \\ Changes}

\section{FY 1995 Actual Milestones}

Continued refinement of high performance

computer-based environments to include access to state-of-the-art massively parallel computers, new algorithms and software specifically tailored for use on these systems, and updated interfaces with high speed networks.

Access to resource center facilities available to scientists nationally to conduct drug design research.

Tested the ability to predict binding constants of known molecule-drug combinations, for example, a known HIV protease-drug inhibitor.

Use of new methods for calculating solvation energies to determine the solubilities of candidate pharmaceuticals prior to chemical synthesis.

the pharmaceutical industry.

Sung to become quantitative. project grants.

\section{Budget (\$ M)}

\begin{tabular}{|l|l|}
\hline FY 95 Act & 2.50 \\
\hline FY 96 Pres. & 2.20 \\
\hline FY 96 Est. & 2.20 \\
\hline FY 97 Rqst. & 2.20 \\
\hline
\end{tabular}

Program

Component

Areas

FY 96 FY 97

\begin{tabular}{|l|l|l|}
\hline HECC & 2.20 & 2.20 \\
\hline LSN & & \\
\hline HCS & & \\
\hline HuCS & & \\
\hline ETHR & & \\
\hline
\end{tabular}

through high speed networks will enable

structure-based design of drugs to become a reality

Design of new molecule-drug combinations usin capability to predict protein-drug binding energies.

Work on calculation of solvation energies

should uncover overestimates in certain binding

energies, e.g., hydrogen binding. New potential

functions must be developed for biomolecular

Support new investigator-initiated research

FY 1997 Agency Request Milestones

Continue use of high performance computer

resources accessible through high speed network support structure- based drug design.

Improve methods to efficiently and accurately

Enhance simulations of complex protein-drug nteractions on/in cellular membranes.

Support new investigator-initiated research project grants.

\section{Agency Ties}

\begin{tabular}{|c|c|}
\hline DARPA & \\
\hline NSF & Partner \\
\hline DOE & \\
\hline NASA & \\
\hline NIH & \\
\hline NSA & \\
\hline NIST & \\
\hline NOAA & \\
\hline EPA & \\
\hline ED & \\
\hline AHCPR & \\
\hline VA & \\
\hline
\end{tabular}

December 20, 1996 


\begin{tabular}{|c|c|c|c|c|c|}
\hline $\mathrm{NIH}$ & NCRR Modeling/Simulation & Budget Code: & & & \\
\hline \multirow{8}{*}{\multicolumn{3}{|c|}{$\begin{array}{l}\text { As scientists strive to understand increasingly more complex biomedical processes, the computer requirements, both hardware and software, needed to } \\
\text { model and simulate these processes increase in performance and complexity. These requirements extend to network capabilities which will be required to } \\
\text { carry increasingly more data per unit time. The research resource centers are the focus for NCRR supported simulation/modeling high performance } \\
\text { computing activities. Areas of interest include simulations of subjects as small as molecules and as large as the entire body--cells, tissues, organs, and organ } \\
\text { systems. In addition are epidemiological models, especially for pressing health problems such as AIDS and cardiovascular disease. }\end{array}$}} & \multicolumn{3}{|c|}{ Budget (\$ M) } \\
\hline & & & \multicolumn{2}{|c|}{ FY 95 Act } & 4.80 \\
\hline & & & \multicolumn{2}{|c|}{ FY 96 Pres. } & 5.80 \\
\hline & & & \multicolumn{2}{|c|}{ FY 96 Est. } & 5.80 \\
\hline & & & \multicolumn{2}{|c|}{ FY 97 Rqst. } & 5.30 \\
\hline & & & \multicolumn{3}{|c|}{$\begin{array}{l}\text { Program } \\
\text { Component } \\
\text { Areas }\end{array}$} \\
\hline & & & \multicolumn{2}{|c|}{ FY 96} & FY 97 \\
\hline & & & HECC & 4.50 & 4.50 \\
\hline \multicolumn{3}{|l|}{\begin{tabular}{|c|} 
Milestone \\
Changes \\
\end{tabular}} & LSN & 0.10 & 0.10 \\
\hline FY 1995 Actual Milestones & FY 1996 Estimated Milestones & FY 1997 Agency Request Milestones & HCS & & \\
\hline \multirow{15}{*}{$\begin{array}{l}\text { Development of new programs specifically } \\
\text { tailored for use on massively parallel computer } \\
\text { systems to achieve very high performance on large } \\
\text { biomolecular systems, such as proteins embedded in } \\
\text { lipid membranes. } \\
\text { Created combined transport and metabolism } \\
\text { models to understand the dynamics of substances } \\
\text { such as oxygen or metabolites in whole organs. } \\
\text { Significant progress towards the realization of a safe, } \\
\text { reliable hemoglobin-based blood substitute based on } \\
\text { simulations of complex NMR data from hemoglobin. } \\
\text { Extended the Monte Carlo models to cover even } \\
\text { smaller populations such as cells. } \\
\text { Began new initiatives in simulation and } \\
\text { modeling. }\end{array}$} & \multirow{15}{*}{$\begin{array}{l}\text { It is estimated that simulations of large } \\
\text { molecular systems in excess of } 100,000 \text { atoms will } \\
\text { be visualized and interactively controlled through } \\
\text { the integration of improved parallel algorithms, } \\
\text { faster numerical methods for longer integration } \\
\text { steps, and the new generations of massively parallel } \\
\text { computers. } \\
\text { Capability to realistically model oxygen } \\
\text { transport and metabolism for the whole body. } \\
\text { Enhanced Monte Carlo models with the } \\
\text { capability to provide dynamic visualization of } \\
\text { epidemiological data. } \\
\text { Extend initiatives in simulation and modeling. } \\
\text { Progress from new initiatives begun in FY } 1995 .\end{array}$} & \multirow{15}{*}{$\begin{array}{l}\text { Continued simulation of increasingly large } \\
\text { molecular systems. } \\
\text { Enhanced models of cells and organs, e.g., } \\
\text { Peskin heart model. } \\
\text { Increased interaction between NIH and NSF } \\
\text { modeling centers via internet. }\end{array}$} & HuCS & 1.20 & 0.70 \\
\hline & & & ETHR & & \\
\hline & & & \multicolumn{3}{|c|}{ Agency Ties } \\
\hline & & & DARPA & \multicolumn{2}{|c|}{ Partner } \\
\hline & & & NSF & \multicolumn{2}{|c|}{ Partner } \\
\hline & & & DOE & & \\
\hline & & & NASA & \multicolumn{2}{|c|}{ Partner } \\
\hline & & & $\mathrm{NIH}$ & \\
\hline & & & NSA & \\
\hline & & & NIST & \\
\hline & & & NOAA & \\
\hline & & & $\mathrm{EPA}$ & \\
\hline & & & ED & \\
\hline & & & AHCPR & \\
\hline & & & VA & \multicolumn{2}{|l|}{+} \\
\hline
\end{tabular}


In certain areas of basic research, the ability to visualize large amounts of data in a 'natural environment is often crucial. In clinical care the ability to 'see' deep inside the body, especially the brain, can determine whether a surgical procedure or other therapeutic intervention can be successfully performed with minimum trauma to the patient. These fundamental requirements are increasingly being provided by an evolving technology known as virtual reality or virtual environments. Among requirements for effective virtual reality is the need to provide realistic images--visualization--in real time, that is, these images change in a realistic fashion according to where the observer is looking in real time. All but the most simple cases require high performance computer capability. The NCRR effort in virtual reality is focused on applications for, scientific instruments via basic research, molecular visualization, and well defined opportunities to support surgical and other therapeutic interventions such as radiation planning.

\section{Milestone In FY 96 "Explore feasibility of establishing 'virtual laboratory testbeds` focused on important biomedical research problems" was added.}

\section{Changes}

FY 1995 Actual Milestones

Extended the nanomanipulator approach to an atomic force microscope which will permit experimentation on biologically interesting macromolecules.

Extended telemicroscopy using network capabilities.

Extended the room filling molecule technology permit more sophisticated interaction and better

wayfinding, such as backbone high-lighting and the ability to identify residues at will.

Enabled chemists to use a new technology

force-display arm of high resolution, accuracy, and

frequency response for molecular docking and foldin studies.

Installed virtual environment technology for brain behavior research, including the addition of virtual force capability.

Began new activities through

investigator-initiated research project grant support

\section{FY 1996 Estimated Milestones}

Obtain preliminary results on the value of coupling virtual visualization capabilities to instruments such as the atomic force microscope; access over wideband networks.

Molecular scientists to experiment with an improved version of the room-filling molecule technology.

Establish preliminary utility of force-arm technology for molecular docking and folding studies.

Integrate kinetic and virtual displays to permit dissociation of visual and vestibular cues in the

course of natural behavior for brain behavior research.

Explore feasibility of establishing 'virtual laboratory testbeds' focused on important biomedical research problems.

Progress in specific basic and clinical research applications initiated in FY 1995.

\section{FY 1997 Agency Request Milestones}

Refine prototype systems of virtual

environments for scientific instruments.

Demonstrate and evaluate new applications for these prototypes.

Establish two or three virtual laboratory testbeds (possibly with NSF and DARPA as partners).

Continue to develop and integrate kinetic and virtual environments to support cognitive neuroscience research.
Budget (\$ M)

\begin{tabular}{|c|r|}
\hline FY 95 Act & 4.00 \\
\hline FY 96 Pres. & 4.20 \\
\hline FY 96 Est. & 4.20 \\
\hline FY 97 Rqst. & 7.70 \\
\hline
\end{tabular}

Program

Component

Areas

FY 96 FY 97

HECC

\begin{tabular}{|l|l|l|}
\hline LSN & & \\
\hline HCS & & \\
\hline HuCS & 4.20 & 7.70 \\
\hline ETHR & & \\
\hline
\end{tabular}

\section{Agency Ties}

\begin{tabular}{|c|c|}
\hline DARPA & Partner \\
\hline NSF & Partner \\
\hline DOE & \\
\hline NASA & \\
\hline NIH & \\
\hline NSA & \\
\hline NIST & \\
\hline NOAA & \\
\hline EPA & \\
\hline ED & \\
\hline AHCPR & \\
\hline VA & \\
\hline
\end{tabular}

December 20, 1996 


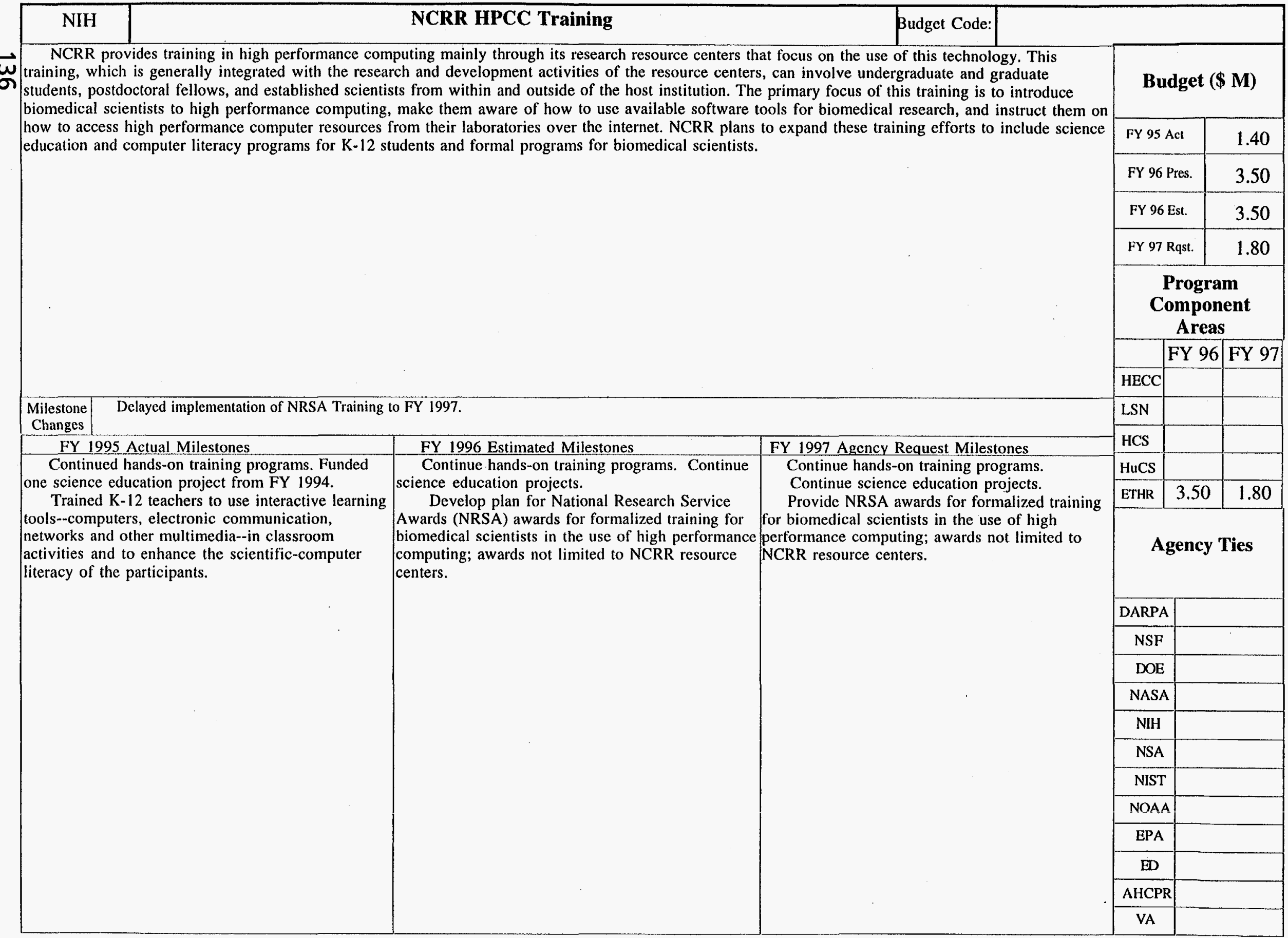




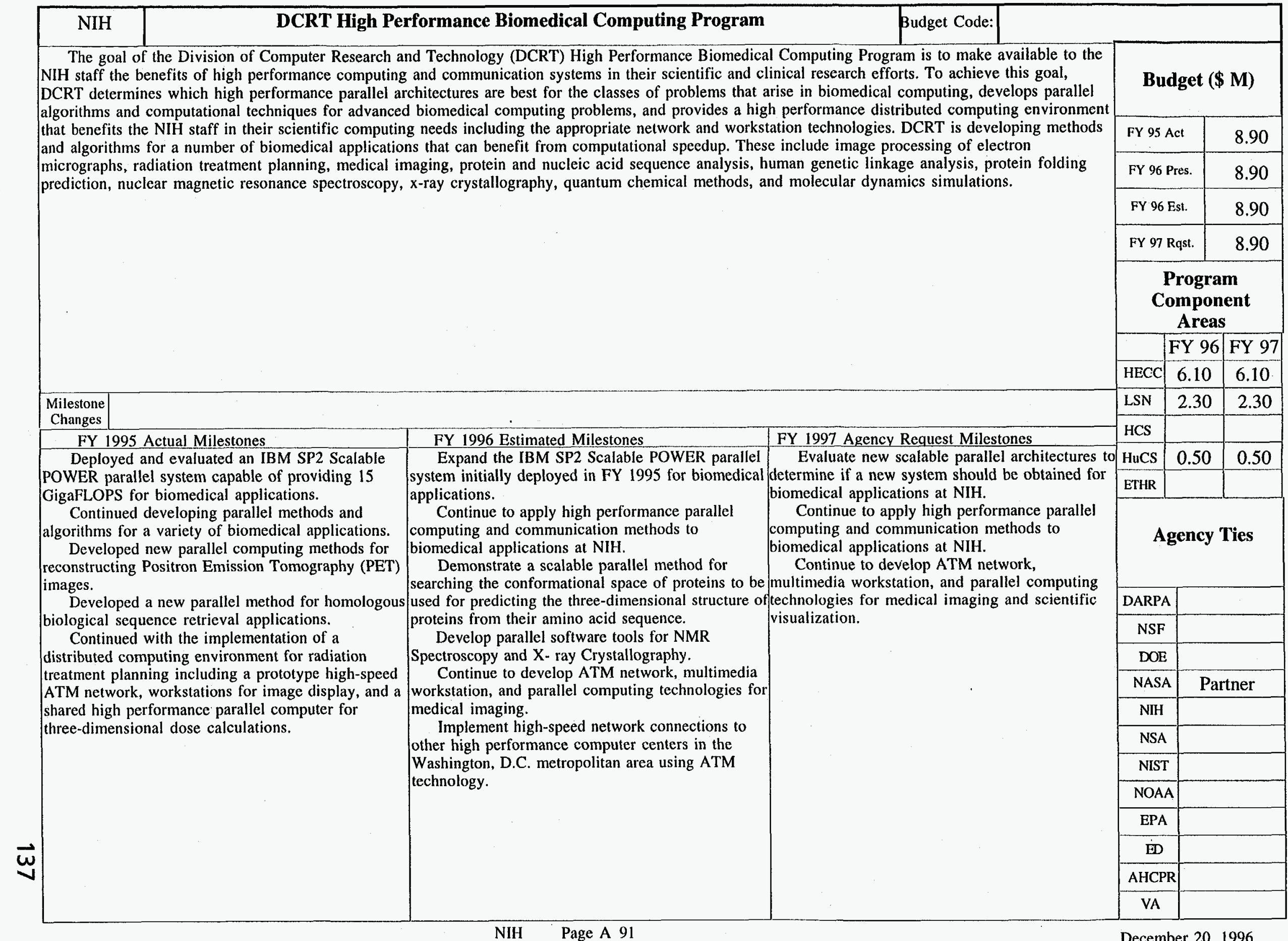




\begin{tabular}{|c|c|c|c|c|c|c|}
\hline \multicolumn{3}{|c|}{ NCI Frederick Biomedical Supercomputing Center } & \multicolumn{4}{|l|}{ Budget Code: } \\
\hline \multirow{8}{*}{\multicolumn{4}{|c|}{$\begin{array}{l}\text { The NCI Frederick Biomedical Supercomputing Center's (FBSC) purpose is to provide high performance computing dedicated and available to the entire } \\
\text { biomedical scientific community to develop basic knowledge for the diagnosis, treatment, understanding and prevention of cancer and other diseases. It } \\
\text { employs advanced techniques in a fully integrated environment of workstations, mid-level, supercomputer and massively parallel computers connected by } \\
\text { networks. Activities are concentrated in those areas of biomedical research computation that are too demanding to be pursued on conventional or immature } \\
\text { computers. Primary concerns are structure determination by x-ray and magnetic resonance, structure prediction of nucleic acids and proteins, computational } \\
\text { biochemistry and problems that arise from modern molecular biology. Genomic sequence analysis, molecular mechanics, ab initio chemistry, linkage } \\
\text { analysis, image analysis and mathematical modeling are primary problem areas. High production algorithms are adapted to vector-multiprocessor and } \\
\text { massively parallel systems, entirely new algorithms are developed and leading-edge computer science discoveries from the areas of computer vision, } \\
\text { robotics, deterministic and non-deterministic, algorithms. }\end{array}$}} & \multicolumn{3}{|c|}{ Budget $(\$ \mathbf{M})$} \\
\hline & & & & \multicolumn{2}{|c|}{ FY 95 Act } & 5.40 \\
\hline & & & & \multicolumn{2}{|c|}{ FY 96 Pres. } & 5.40 \\
\hline & & & & \multicolumn{2}{|c|}{ FY 96 Est. } & 5.90 \\
\hline & & & & \multicolumn{2}{|c|}{ FY 97 Rqst. } & 5.90 \\
\hline & & & & \multicolumn{3}{|c|}{$\begin{array}{c}\text { Program } \\
\text { Component } \\
\text { Areas }\end{array}$} \\
\hline & & & & \multicolumn{2}{|c|}{ FY 96} & FY. 97 \\
\hline & & & & HECC & 3.60 & 3.60 \\
\hline \begin{tabular}{|r|} 
Milestone \\
Changes
\end{tabular} & \multicolumn{3}{|c|}{ Provide state of the art capabilities in a fully integrated high performance computing center. } & \multirow{2}{*}{\begin{tabular}{|l|} 
LSN \\
HCS \\
\end{tabular}} & 1.39 & \multirow{2}{*}{$\begin{array}{l}1.39 \\
0.14 \\
\end{array}$} \\
\hline FY 1 & 995 Actual Milestones & FY 1996 Estimated Milestones & FY 1997 Agency Request Milestones & & 0.14 & \\
\hline \multirow{15}{*}{\multicolumn{2}{|c|}{$\begin{array}{l}\text { Used computational and structure-based } \\
\text { techniques to design novel inhibitors of enzymes } \\
\text { implicated in AIDS, cancer, and tuberculosis. } \\
\text { Adapted algorithms for motif detection in } \\
\text { genomes and molecular structures to several parallel } \\
\text { computer architectures. } \\
\text { Implemented client server technology to improve } \\
\text { the system- investigator in interfaces. }\end{array}$}} & \multirow{15}{*}{$\begin{array}{l}\text { Continue to attract important areas of research } \\
\text { for the application of high performance computing to } \\
\text { health-related basic research. } \\
\text { Expand activities in the use of distributed, } \\
\text { heterogeneous computing for problems such as } \\
\text { molecular mechanical calculations. } \\
\text { Provide expanded scalable computing such as } \\
\text { workstation farms, and distributed batch computing } \\
\text { queues for rapid throughput of smaller tasks. } \\
\text { Evaluate the advanced computational hardware } \\
\text { and software available for productive, forefront } \\
\text { application, especially those architectures showing } \\
\text { promise of proven balanced performance in a } \\
\text { research production environment. }\end{array}$} & \multirow{15}{*}{$\begin{array}{l}\text { Expand activities in high performance } \\
\text { computing applications to molecular structure } \\
\text { prediction of nucleic acids and proteins. } \\
\text { Apply computational chemistry to } \\
\text { understanding of drug interactions with enzymes } \\
\text { and nucleic acids as a basis for discovery of new } \\
\text { drugs. } \\
\text { Provide support for increasing numbers of users } \\
\text { in extramural and intramural research in } \\
\text { biomedical computing. }\end{array}$} & HuCs & 0.28 & 0.28 \\
\hline & & & & ETHR & 0.49 & 0.49 \\
\hline & & & & \multicolumn{3}{|c|}{ Agency Ties } \\
\hline & & & & DARPA & & \\
\hline & & & & NSF & & \\
\hline & & & & DOE & & \\
\hline & & & & NASA & & \\
\hline & & & & NIH & & \\
\hline & & & & NSA & Par & tner \\
\hline & & & & NIST & Par & tner \\
\hline & & & & NOAA & & \\
\hline & & & & EPA & & \\
\hline & & & & ED & & \\
\hline & & & & AHCPR & & \\
\hline & & & & VA & & \\
\hline
\end{tabular}




\begin{tabular}{|c|c|c|}
\hline NIH & NCI High Speed Networking and Distributed Conferencing & Budget Code: \\
\hline
\end{tabular}

Improve access methodologies for diverse members of the biomedical research community to the entire computational infrastructure of the NCI Frederick Biomedical Supercomputing Center (FBSC) by improving the data communications and networking infrastructure through the implementation of evolving networking technologies and high speed interfaces. Initiatives in this area cover a wide variety of data communications technologies including local area networking, wide area networking, requirements for multimedia data transmission, dedicated specialized high speed interfaces for local computer to computer connections (e.g. high speed crossbar switches, HIPPI, fiber channel, etc.), and the use of evolving data communications standards such as ATM and SONET.

\section{Milestone}

Changes

FY 1995 Actual Milestones

Acquired and deployed high speed interconnects for the major components of the FBSC.

Established connections to other laboratories of the NIH and biomedical community for high speed data communication.

Refined and developed software for access to experimental and computational data from world-wide sources.

\section{FY 1996 Estimated Milestones}

Bring demonstrated capabilities in high performance computing to health research

organizations of the public and private sector in open and efficient manner.

Develop and expand high speed links between remote sites for access of the intramural and extramural biomedical research community for video and data conferencing, distributed

collaborations and distributed database and

computing.
FY 1997 Agency Request Milestones

Expand availability of visual and voice interactions between biomedical computing researchers and projects.

\section{Budget (\$ M)}

\begin{tabular}{|c|c|}
\hline FY 95 Act & 2.00 \\
\hline FY 96 Pres. & 1.70 \\
\hline FY 96 Est. & 1.34 \\
\hline FY 97 Rqst. & 1.31 \\
\hline
\end{tabular}

Program

Component

Areas

FY 96 FY 97

\begin{tabular}{|l|l|l|}
\hline HECC & 0.20 & 0.20 \\
\hline LSN & 0.72 & 0.72 \\
\hline
\end{tabular}

\begin{tabular}{|l|l|l|}
\hline LSN & 0.72 & 0.72 \\
\hline HCS & & \\
\hline HuCS & 0.34 & 0.34 \\
\hline ETHR & 0.08 & 0.05 \\
\hline
\end{tabular}

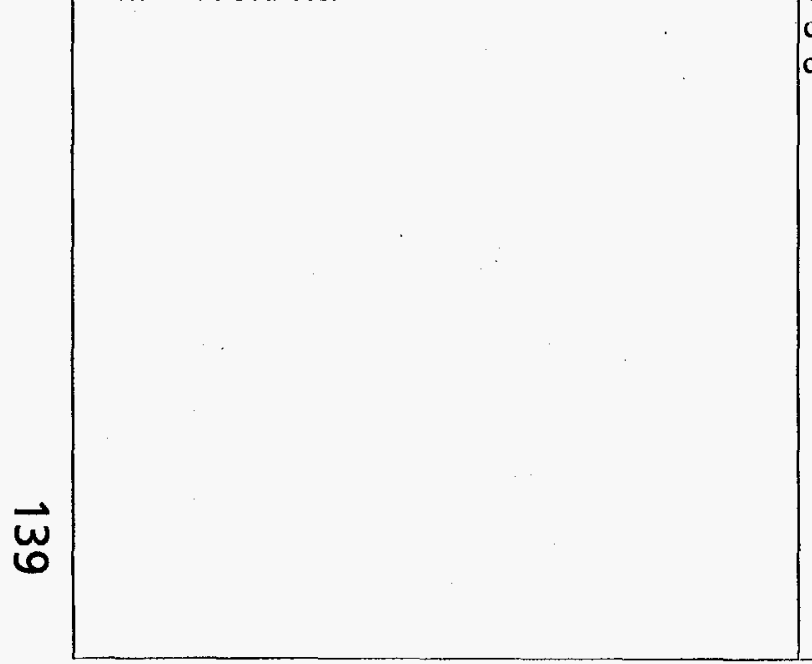

Agency Ties

\begin{tabular}{|c|c|}
\hline DARPA & \\
\hline NSF & \\
\hline DOE & \\
\hline NASA & \\
\hline NIH & \\
\hline NSA & \\
\hline NIST & \\
\hline NOAA & \\
\hline EPA & \\
\hline ED & \\
\hline AHCPR & \\
\hline VA & \\
\hline
\end{tabular}

December 20, 1996 


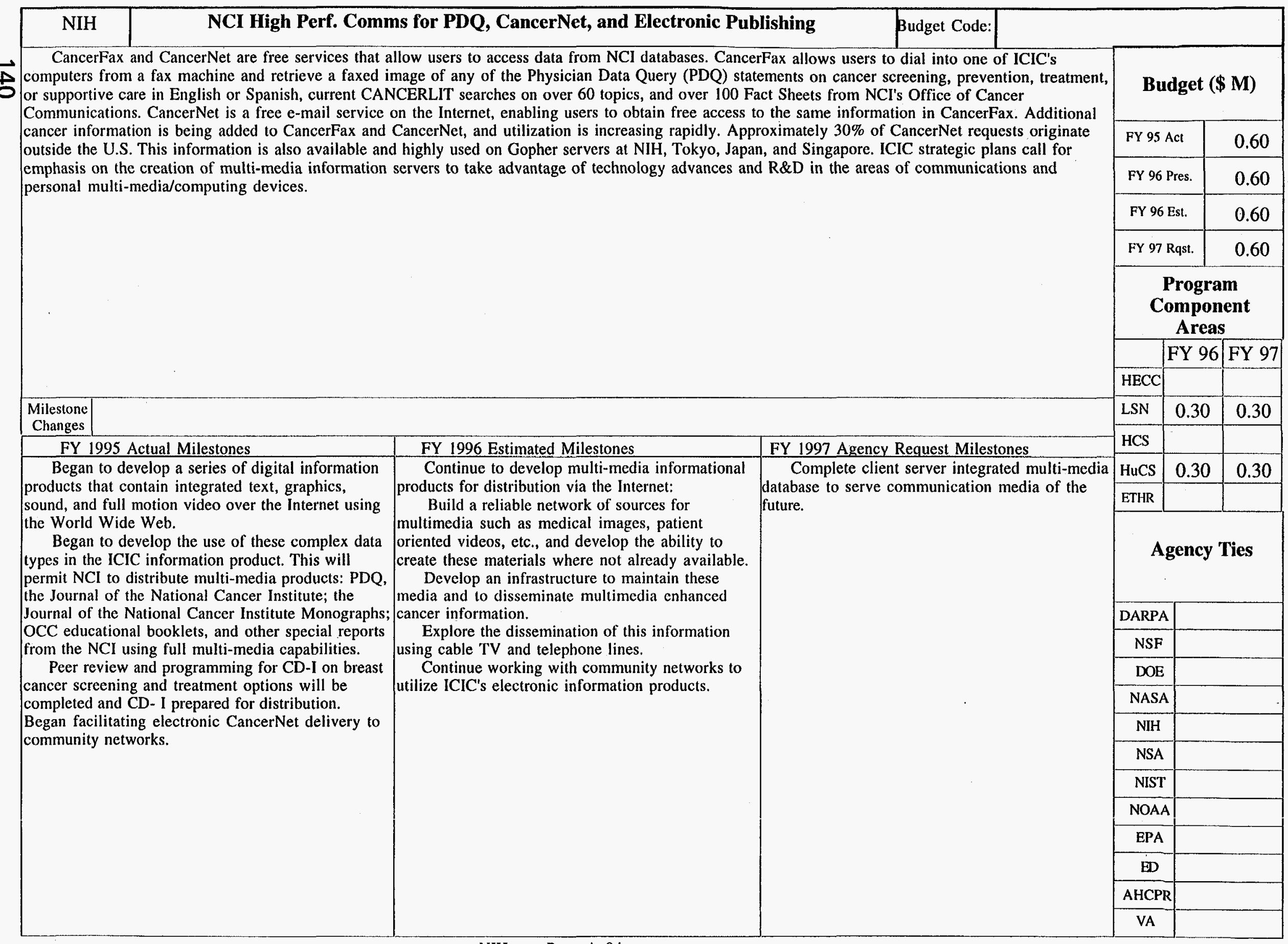




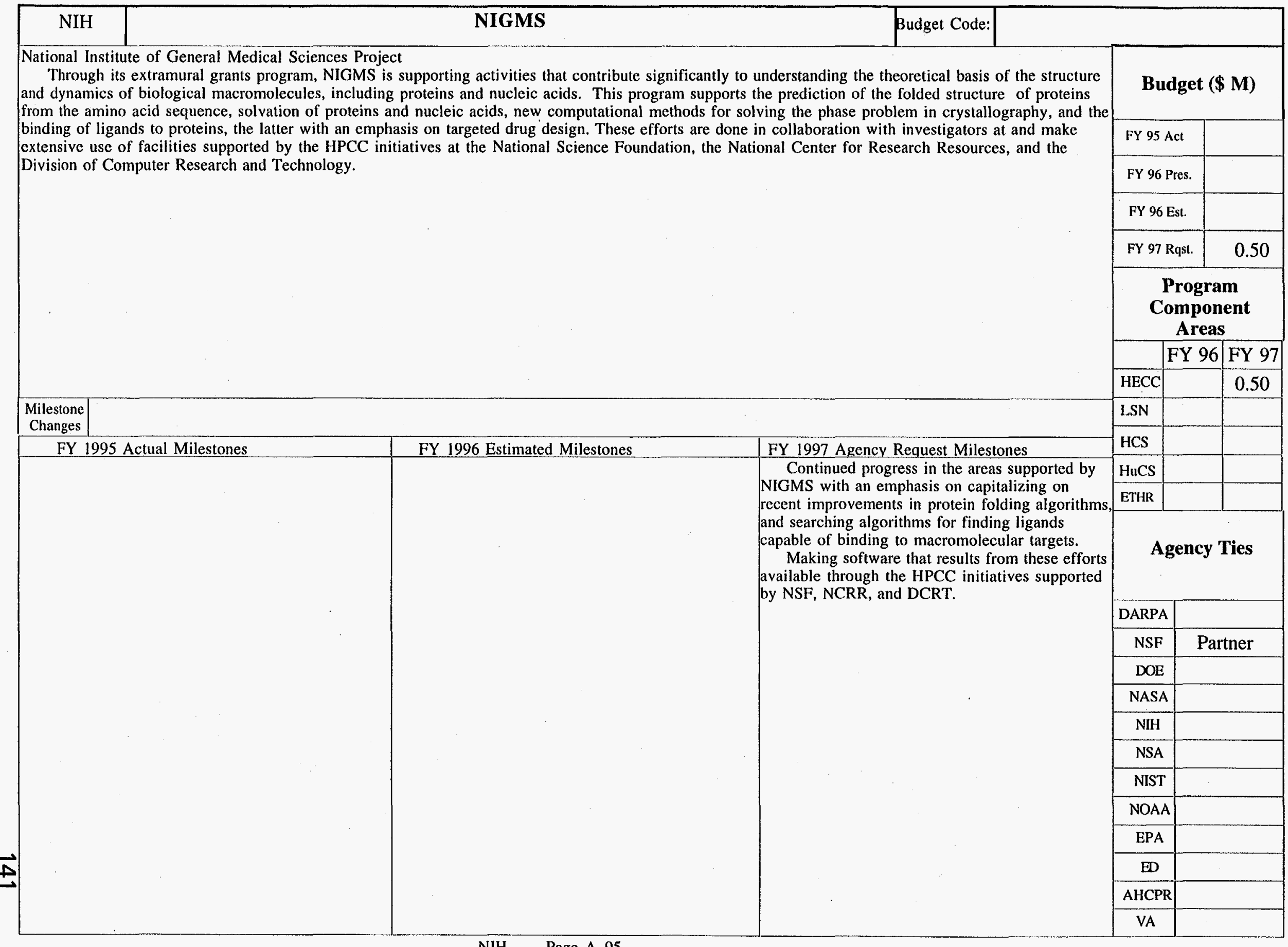




\section{National Security Agency}

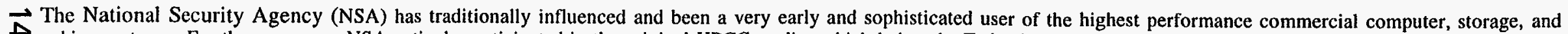

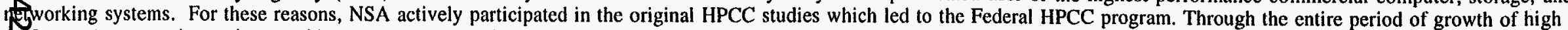

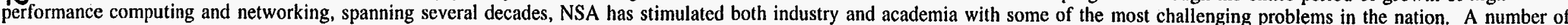

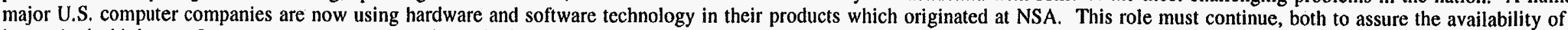

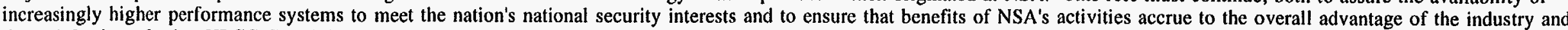
the satisfaction of other HPCC Grand Challenges.

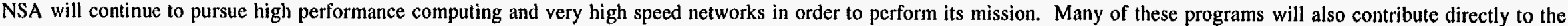
overall goals of HPCC. NSA sponsors divisions of the Institute for Defense Analyses (an FFRDC) to do most of this research.

Results of programs and external drivers have led to revised priorities and funding levels for the supercomputing, superconducting, and very high speed research programs.

\begin{tabular}{|c|c|c|c|c|c|c|c|c|c|c|c|}
\hline \multirow[b]{2}{*}{ Program Activity } & \multirow[b]{2}{*}{ Budget Account Code } & \multirow{2}{*}{$\begin{array}{l}\text { Partner/User } \\
\text { Agencies }\end{array}$} & \multicolumn{4}{|c|}{ Budget (BA, \$ M) } & \multicolumn{5}{|c|}{ HPCCPCAs by 1997 Pres. Request } \\
\hline & & & $\begin{array}{l}\text { FY } 95 \\
\text { Actual } \\
\end{array}$ & $\begin{array}{l}\text { FY } 96 \\
\text { Pres. } \\
\end{array}$ & $\begin{array}{c}\text { FY } 96 \\
\text { Est. }\end{array}$ & $\begin{array}{l}\text { FY } 97 \\
\text { Rqst. }\end{array}$ & HECC & LSN & HCS & HuCS & ETHR \\
\hline Supercomputing Research & & \begin{tabular}{|l} 
ARPA, \\
NASA, NIH \\
\end{tabular} & 25.90 & 27.40 & 27.48 & 23.43 & 23.43 & & & & \\
\hline Supercomputing Research (continued) & & $\begin{array}{l}\text { ARPA, } \\
\text { NASA, NIH } \\
\end{array}$ & & & & & & & & & \\
\hline Superconducting Research & & NASA & 2.00 & 2.00 & 2.00 & 2.50 & 2.50 & & & & \\
\hline Very High Speed Networking & & ARPA & 3.50 & 3.00 & 3.00 & 3.50 & & 3.50 & & & \\
\hline Very High Speed Networking (continued) & & ARPA & & & & & & & & & \\
\hline Secure Operating System Development & & NIST & 5.70 & 4.70 & 4.70 & 4.50 & & & 4.50 & & \\
\hline High Speed Data Protection Electronics & & ARPA, NASA & 2.60 & 2.70 & 2.70 & 2.80 & & & 2.80 & & \\
\hline Technology Based Training & & & 0.23 & 0.23 & 0.15 & & & & & & \\
\hline & & Totals: & 39.93 & 40.03 & 40.03 & 36.73 & 25.93 & 3.50 & 7.30 & & \\
\hline
\end{tabular}




\section{National Security Agency}

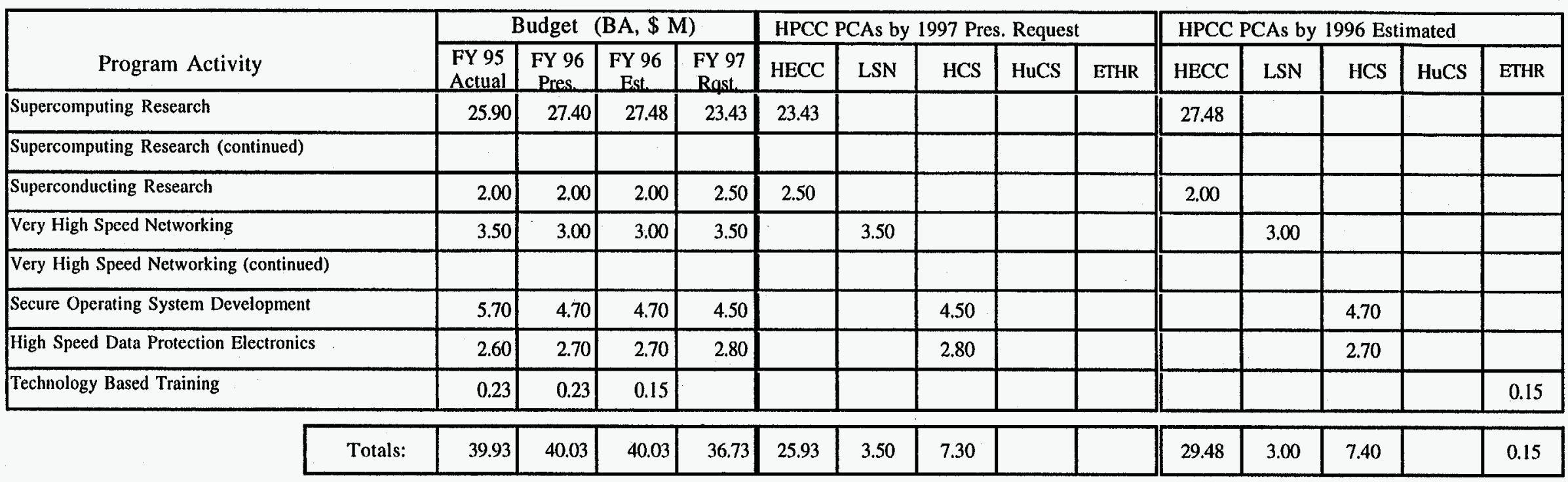




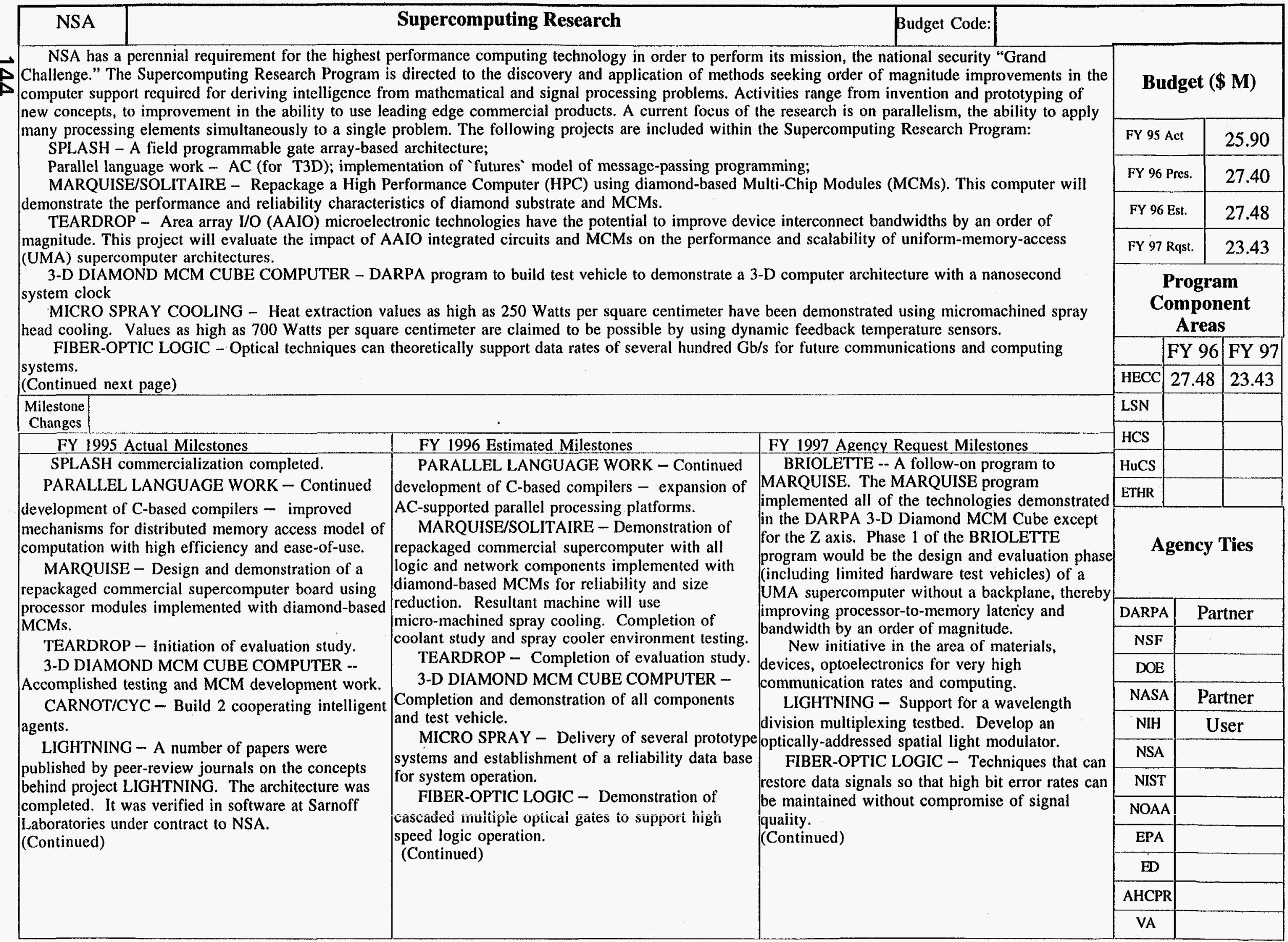




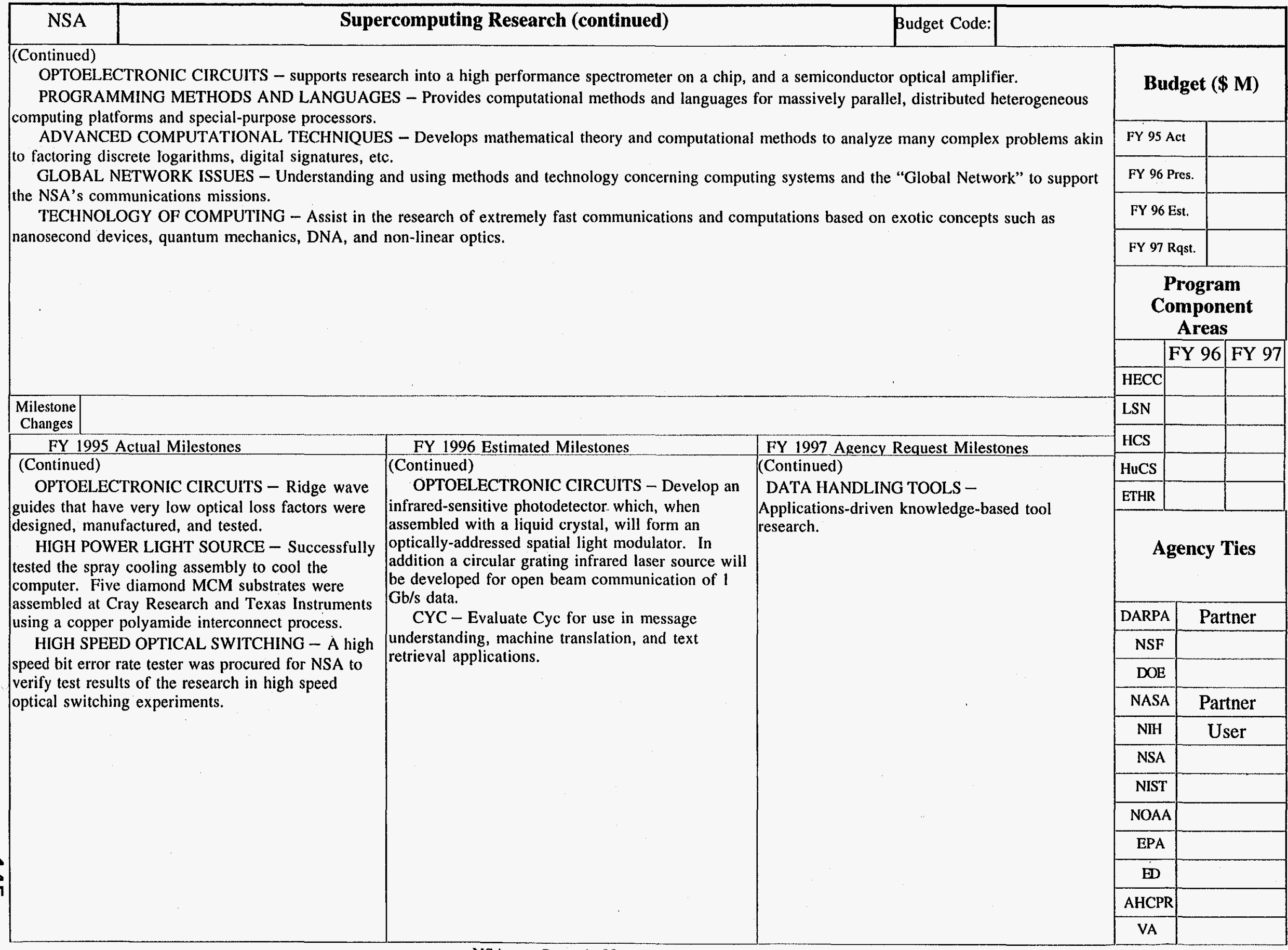




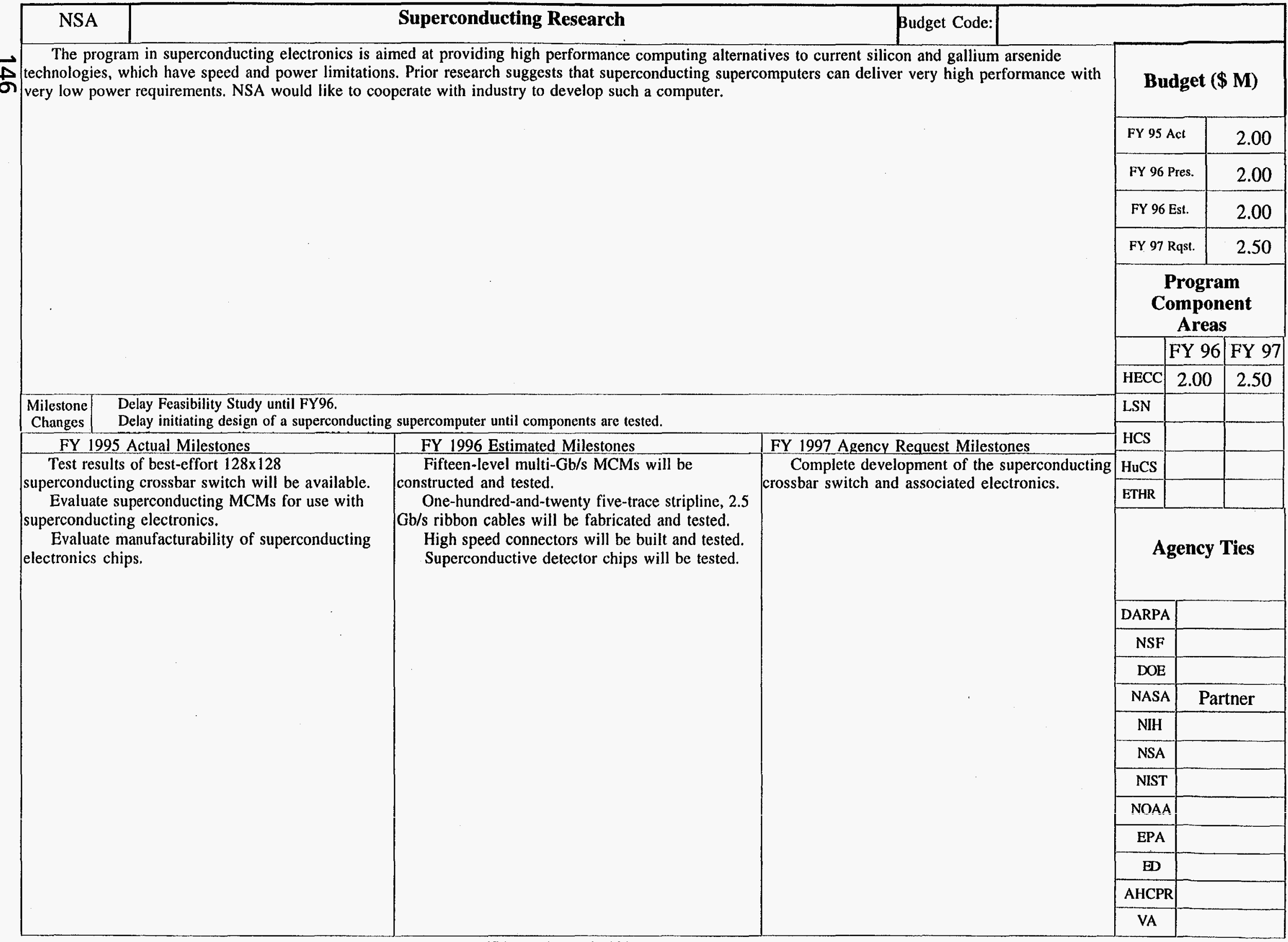




\begin{tabular}{|c|c|c|}
\hline NSA & Very High Speed Networking & Budget Code: \\
\hline
\end{tabular}

The National Security Agency has a perennial requirement for the fastest networking technology in order to perform its mission, the national security 'Grand Challenge.' The Very High Speed Networking Program will provide NSA a high performance network infrastructure characterized by both multi-gigabit per second trunking speeds and the ability to support sustained data flows of at least hundreds of megabits per second each now, and ultimately multi-gigabits per second each.

Milestone Delay demonstration of electronic switching of individual data flows at $2.4 \mathrm{~Gb} / \mathrm{s}$ data streams to FY97. Changes

$$
\text { FY } 1995 \text { Actual Milestones }
$$

Install an in-house optical crossbar network testbed to explore high-speed optical network architectures and optical network management techniques.

Install in-house ATM/SONET testbed equipment and begin experimentation on sustained data flows a hundreds of megabits per second.

Initiate development of wave division optical switching and multiplexing devices to support $\mathrm{Gb} / \mathrm{s}$ data rates.

Conduct soliton research to explore application high-speed networks by developing soliton source and logic gates.

Continue collaboration with industry on the development of high-speed (electronic and optical) prototype switching equipment.

Continue collaboration with industry on the development of operation, administration and management protocols for optically switched networks.

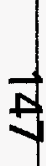

FY 1996 Estimated Milestones

Demonstrate electronic switching of individual

data flows at $650 \mathrm{Mb} / \mathrm{s}$ data streams.

Initiate resolution of discovered ATM/IP

incompatibilities, and the development of IP routers

$\mathrm{Gb} / \mathrm{s}$, to achieve a truly $\mathrm{Gb} / \mathrm{s}$ Internet.

Determine the characteristics of end-to-end

protocols that are effective in a $\mathrm{Gb} / \mathrm{s}$ Internet.

Continue the development of optical switching

and wave division multiplexing devices to support up to $10 \mathrm{~Gb} / \mathrm{s}$ data rates.

ntegrate and experiment with out of band

network control in optical crossbar network.

Test multi-wavelength signals on installed fiber for multi-wavelength overlay on DC Metro area

ATM/SONET testbed. Ultimately (1999), the full multi-wavelength testbed will begin to show

transition path from ATM/SONET cell switching to wave division switching and wave conversion network.

(Continued)
FY 1997 Agency Request Milestones

Demonstrate electronic switching of individua data flows at $2.4 \mathrm{~Gb} / \mathrm{s}$ data streams.

Demonstrate $\mathrm{a} \mathrm{Gb} / \mathrm{s}$ Internet capability by connecting $\mathrm{Gb} / \mathrm{s}$ ATM networks with a $\mathrm{Gb} / \mathrm{s} \mathrm{IP}$

router, with individual data flow of $2.4 \mathrm{~Gb} / \mathrm{s}$ over same.

Continue the development of optical switching and wave division multiplexing devices to suppor up to $10 \mathrm{~Gb} / \mathrm{s}$ data rates.

Transition out of band network control for ATM to multi-wavelength overlay terminal devices in anticipation of FY98-99 testing of multi-wavelength switching devices on the DC-area testbed multi-wavelength overlay.

Begin significant implementation and multi-network testing over DC-area's ATM testbed and attached networks of interoperable network management.

(Continued)

\begin{tabular}{|c|c|}
\hline DARPA & Partner \\
\hline NSF & \\
\hline DOE & \\
\hline NASA & \\
\hline NIH & \\
\hline NSA & \\
\hline NIST & \\
\hline NOAA & \\
\hline EPA & \\
\hline ED & \\
\hline AHCPR & \\
\hline VA & \\
\hline
\end{tabular}

December 20, 1996 rogram

Component

Areas

\begin{tabular}{|l|l|l|}
\hline & FY 96 & FY 97 \\
\hline HECC & & \\
\hline LSN & 3.00 & 3.50 \\
\hline HCS & & \\
\hline HuCS & & \\
\hline ETHR & & \\
\hline
\end{tabular}

Agency Ties 


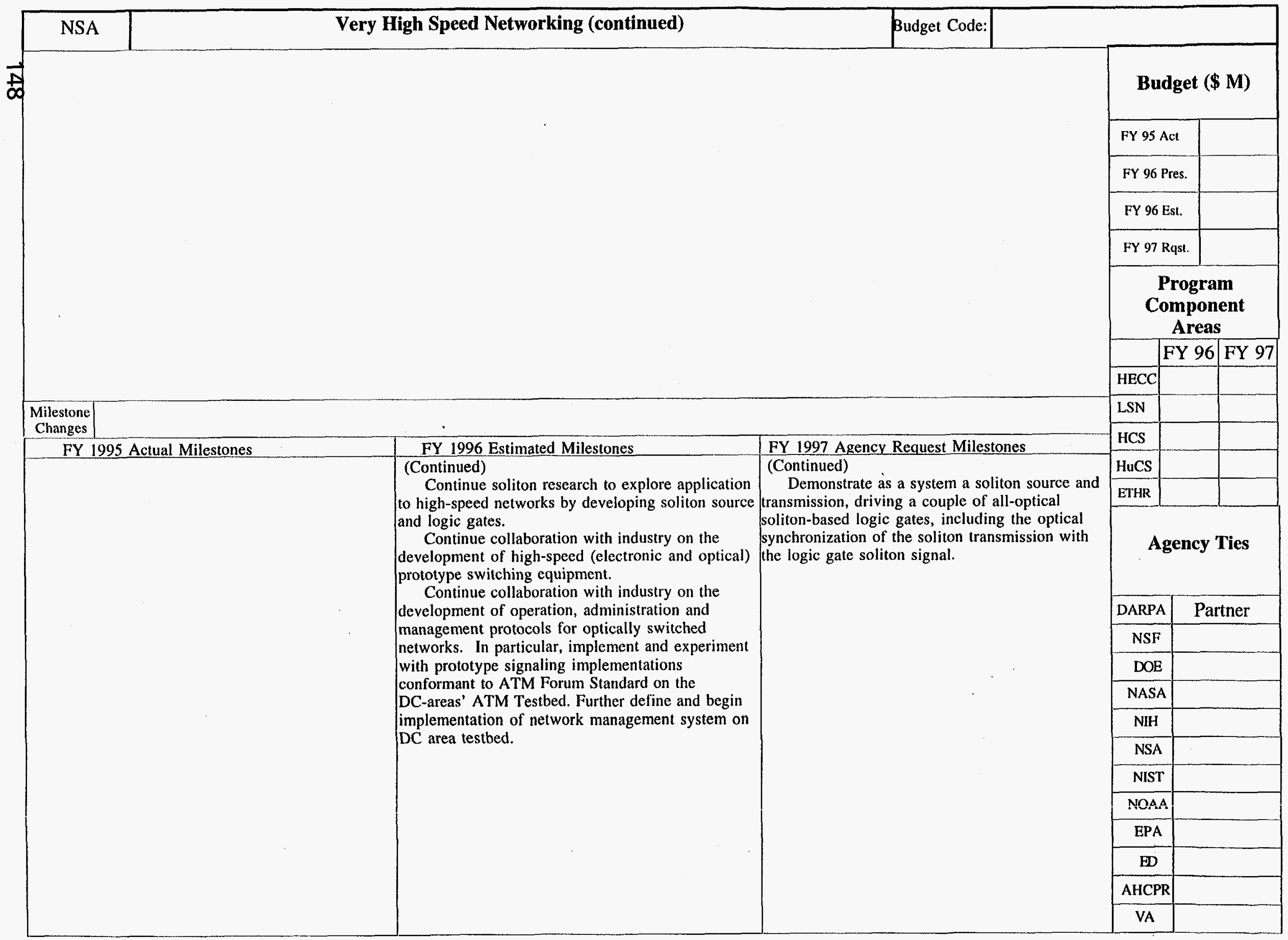


NSA is responsible for researching solutions to ensure the security of the nation's networks and distributed systems. In collaboration with NIST and DISA NSA is identifying issues and researching security policy-flexible, cost- effective ways to ensure the security of both government and commercial enterprise systems. The Synergy research program at NSA is developing an 'open architecture' along with secure distributed system prototypes based upon security policy-flexible, operating system microkernels. Synergy will provide a means for commercial vendors to address a wide variety of security markets with a single architecture, thus lowering everyone's costs. Synergy can be the foundation on which security solutions for major initiatives can be built, whether addressing government-peculiar needs or commercial-enterprise needs, from the Defense Department Global Grid to the National Information Infrastructure. Synergy will integrate the INFOSEC research work in computer misuse and anomaly detection (audit/intrusion detection), real-time and multimedia, availability, network security management, high speed networking, and secure database management systems.

\section{Budget (\$ M)}

\begin{tabular}{|c|c|}
\hline FY 95 Act & 5.70 \\
\hline FY 96 Pres. & 4.70 \\
\hline FY 96 Est. & 4.70 \\
\hline FY 97 Rqst. & 4.50 \\
\hline
\end{tabular}

Program

Component

Areas

\begin{tabular}{|l|c|c|}
\hline & FY 96 & FY 97 \\
\hline HECC & & \\
\hline LSN & & \\
\hline HCS & 4.70 & 4.50 \\
\hline HuCS & & \\
\hline ETHR & & \\
\hline & &
\end{tabular}

Released Synergy proof of concept software to universities, commercial research labs, and secure system vendors.

Continued university research program to

advance Synergy arch.

Funded 1-3 universities to develop course in secure distributed systems and perform Synergy research.

Initiated internal and external research efforts to mitigate the risks and costs associated with high assurance product implementations of the Synergy architecture.

Developed a centralized security management tool to be integrated with Synergy on a high-speed testbed.

Established a collaborative relationship via CRADA or contract with one or more commercial operating system vendors or consortia, to begin implementing the Synergy architecture in a commercial distributed system.

\section{FY 1996 Estimated Milestones}

Second public release of Synergy software and initial evaluation documentation and experience, to encourage modular system design and evaluation.

Continue research on Synergy architecture to improve security, assurance, performance, availability, and portability in distributed and real-time operating environments.

Demonstrate and make available higher assured versions of security managers and Synergy components.

Continue university research contracts, and ensure successful technology transfer of research results into Synergy architecture development efforts.

Continue collaboration with commercial vendors to encourage the development of Synergy-based systems that can satisfy commercial enterprise security requirements and those of $\mathrm{DoD}$ and the government.

\section{FY 1997 Agency Request Milestones}

Release expanded Synergy prototype includin collaboration with vendors and universities, to system development.

Continue Synergy research to improve the security of distributed operating systems.

Investigate high-assurance security

mechanisms to support future distributed operating

Demonstrate and make available high assurance components for distributed operating systems.

Continue funding university research in the areas of high-assurance operating systems and distributed systems. Integrate results into Synergy security solutions.

Continue working relationships with commercial vendors to influence the design of future commercial architectures. enhancements and improvements derived from influence the design of commercial operating system architectures.

$$
\text { r. }
$$

\section{Agency Ties}

\begin{tabular}{|c|l|}
\hline DARPA & \\
\hline NSF & \\
\hline DOE & \\
\hline NASA & \\
\hline NIH & \\
\hline NSA & \\
\hline NIST & Partner \\
\hline NOAA & \\
\hline EPA & \\
\hline ED & \\
\hline AHCPR & \\
\hline VA & \\
\hline
\end{tabular}

December 20, 1996 


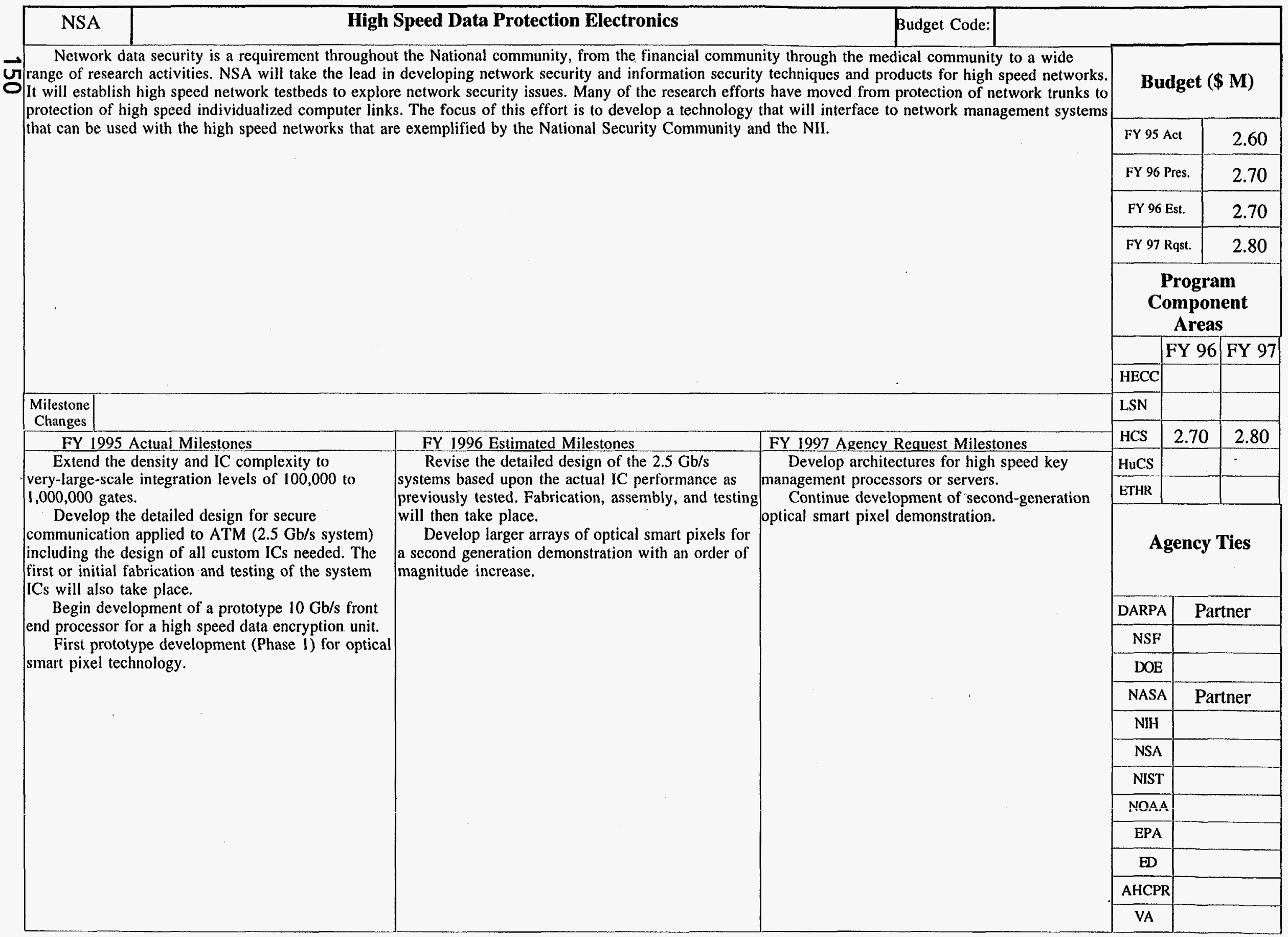




\begin{tabular}{|c|c|c|}
\hline NSA & Technology Based Training & Budget Code: \\
\hline
\end{tabular}

NSA considers lifelong access to education and training for all employees to be highly crucial in order to maintain and enhance their skills. NSA must ensure its viability in a world of rapidly changing technology. Traditional training approaches take too long and are too expensive. Consequently, NSA is developing a skill-based assessment and training model that will use emerging communications and computer techniques to sustain each employee's abilities at the levels required in today's constantly changing workplace. The key ingredient in this approach will be on-demand access to Technology Based Training (TBT) of known quality and relevance delivered directly to the workplace. Delivery over corporate LANs and the Internet, and the possible use of ISDN technology, will be addressed. Although initial offerings would consist largely of text-based TBT courseware, digital video and other media will be accommodated as they become available.

Placing existing TBT courseware on the network is something that can be accomplished quickly, but there are various conceptual, technical and procedural issues that must be addressed before it can be made generally accessible. Foremost among these issues are:

1 -- Definition of the skills needed to perform specific jobs and a process for certifying the efficacy of training modules that address those skills; 2 -- Storage, access, and maintenance of large numbers of TBT courses;

3 -- Cataloging of available courseware in a manner that minimizes the difficulty of identifying suitable courses or training modules;

4 -- Identifying and resolving the interoperability problems in delivering TBT courses over a network to a wide variety of computer terminals;

5 -- Defining and selecting appropriate user interfaces (special effort will be focused on interfaces for home-computer configurations);

6 -- Developing an EDI-based business model bringing market forces to bear on the availability and costs of Technology Based Training.

\section{Milestone}

Changes

FY 1995 Actual Milestones

Perform skill-based certification of approximately

100 existing training modules.

Build user-friendly catalog of available certified courses.

Established'digital warehouses' of TBT on-line via Internet and the NSA corporate network.

Implement pilot of Electronic Payment business model for purchase/delivery of COTS TBT over the INTERNET.
FY 1996 Estimated Milestones

Evaluate, modify, and extend Electronic

Payment business model for online training modules Complete formal guidelines and procedures for creation of certified skill-based training modules.

Design and pilot Individual Development Plan (IDP) database and management software.
Budget (\$ M)

\begin{tabular}{|c|c|c|}
\hline \multicolumn{2}{|c|}{ FY $95 \mathrm{Act}$} & 0.23 \\
\hline \multicolumn{2}{|c|}{ FY 96 Pres. } & 0.23 \\
\hline \multicolumn{2}{|c|}{ FY 96 Est. } & 0.15 \\
\hline \multicolumn{3}{|c|}{ FY 97 Rqst. } \\
\hline \multicolumn{3}{|c|}{$\begin{array}{c}\text { Program } \\
\text { Component } \\
\text { Areas }\end{array}$} \\
\hline & FY 96 & FY 97 \\
\hline \multicolumn{3}{|c|}{ HECC } \\
\hline \multicolumn{3}{|l|}{ LSN } \\
\hline \multicolumn{3}{|l|}{ HCS } \\
\hline \multicolumn{3}{|l|}{ HuCS } \\
\hline ETHR & 0.15 & \\
\hline
\end{tabular}

\section{Agency Ties}

\begin{tabular}{|c|c|}
\hline DARPA & \\
\hline NSF & \\
\hline DOE & \\
\hline NASA & \\
\hline NIH & \\
\hline NSA & \\
\hline NIST & \\
\hline NOAA & \\
\hline EPA & \\
\hline ED & \\
\hline AHCPR & \\
\hline VA & \\
\hline
\end{tabular}

December 20, 1996 


\section{National Institute of Standards and Technology}

Goals of NIST's Program are:

* Apply high performance computing and networking technology to promote improved U.S. product quality and manufacturing performance, to reduce production costs and time-to-market, and to increase competitiveness in international markets;

* Promote the development and deployment of advanced information and communications technology to support the education, research and manufacturing communities and to increase the electronic availability of scientific and engineering data;

* Advance performance measurement methodologies and analytic tools for high performance computing and networking systems, system components, and human-machine interfaces; and to achieve improved system and application program performance;

* Develop efficient algorithms and portable, scalable software for the application of high performance computing systems to industrial problems, and to develop improved methods for the public dissemination of advanced software and documentation; and

* Support, promote, and coordinate the development of voluntary standards that provide interoperability and common user interfaces within the NII, and increase industrial competitiveness.

Objectives of NIST's Program are to accelerate the development and deployment of high performance computing and networking technologies and services required for the National Information Infrastructure, and to apply and test these technologies in a manufacturing environment.

NIST supports and coordinates the development of standards within the Federal government to provide interoperability, common user interfaces to systems, and enhanced security. NIST works with other agencies to promote open system standards and to aid in the commercialization of technology by U.S. industry. NIST promotes the development of communications infrastructure and through information technology research, development, and related activities to enhance basic communications capabilities. Supporting activities include metrology to assess network components for performance, interoperability and conformity; and collaboration with industry via cooperative research and development, sponsorship of workshops, other public fora, and laboratory-based technology demonstrations. NIST develops instrumentation and methodology for performance measurement of scalable, high performance computing and networking systems. Emphasis of the performance measurement component is on the development and use of low perturbation data capture hardware and simplified software-based approaches to performance characterization of these systems and software components.

NIST uses its experience in information technology and manufacturing engineering to accelerate the application of high performance computing and communication technology to manufacturing environments. NIST supports expanded programs in: advanced manufacturing systems integration technologies; development and test of prototype components and interface specifications for manufacturing systems; application of high performance computing and networking technologies to integrate the design and production processes; and testbeds for achieving cost-effective application of advanced manufacturing systems and networks. NIST also addresses key issues in electronic commerce, electronic libraries, and network security. NIST develops algorithms and generic software for advanced scientific, engineering and manufacturing applications. Common elements and techniques are encapsulated in software libraries to promote ease of use and application portability. NIST's Guide to Availabile Ivathematical Sof́tware (GAMís) provides industry and the pubiic with improved electronic access to reusable software.

Note: Three program elements proposed in FY 1996 have been combined for this plan. 


\section{National Institute of Standards and Technology}

\begin{tabular}{|c|c|c|c|c|c|c|c|c|c|c|c|}
\hline \multirow[b]{2}{*}{ Program Activity } & \multirow[b]{2}{*}{ Budget Account Code } & \multirow{2}{*}{$\begin{array}{c}\text { Partner/User } \\
\text { Agencies }\end{array}$} & \multicolumn{4}{|c|}{ Budget (BA, \$ M) } & \multicolumn{5}{|c|}{ HPCC PCAs by 1997 Pres. Request } \\
\hline & & & $\begin{array}{l}\text { FY } 95 \\
\text { Actual } \\
\end{array}$ & $\begin{array}{l}\text { FY } 96 \\
\text { Pres. }\end{array}$ & $\begin{array}{c}\text { FY } 96 \\
\text { Est. }\end{array}$ & $\begin{array}{c}\text { FY } 97 \\
\text { Rast }\end{array}$ & HECC & LSN & HCS & HuCS & ETHR \\
\hline $\begin{array}{l}\text { Development and Dissemination of Scientific Software } \\
\text { for HPCS }\end{array}$ & $\begin{array}{c}\text { STRS for Computer } \\
\text { Sustems }\end{array}$ & $\begin{array}{l}\text { NSF, DOE, } \\
\text { NASA }\end{array}$ & 3.37 & 3.60 & 3.37 & 3.37 & 2.77 & 0.60 & & & \\
\hline Infrastructure for Information Technology & $\begin{array}{l}\text { STRS for Computer } \\
\text { Sustems }\end{array}$ & $\begin{array}{l}\text { ARPA, DOE, } \\
\text { NSA }\end{array}$ & 9.48 & 18.24 & 9.48 & 9.48 & 1.22 & 1.86 & 4.20 & 2.20 & \\
\hline Infrastructure for Information Technology (continued) & $\begin{array}{l}\text { STRS for Computer } \\
\text { Sustems }\end{array}$ & ARPA, DOE, & & & & & & & & & \\
\hline \multirow[t]{2}{*}{ Systems Integration for Manufacturing Applications } & $\begin{array}{l}\text { STRS for Computer } \\
\text { Sustems }\end{array}$ & ARPA, DOE & 10.66 & 12.26 & 10.66 & 10.66 & 1.80 & 0.50 & 1.00 & 7.36 & \\
\hline & & Totals: & 23.51 & 34.10 & 23.51 & 23.51 & 5.79 & 2.96 & 5.20 & 9.56 & \\
\hline
\end{tabular}


National Institute of Standards and Technology

$\vec{\perp}$

\begin{tabular}{|c|c|c|c|c|c|c|c|c|c|c|c|c|c|c|}
\hline \multirow[b]{2}{*}{ Program Activity } & \multicolumn{4}{|c|}{ Budget $(\mathrm{BA}, \$ \mathrm{M})$} & \multicolumn{5}{|c|}{ HPCC PCAs by 1997 Pres. Request } & \multicolumn{5}{|c|}{ HPCC PCAs by 1996 Estimated } \\
\hline & $\begin{array}{l}\text { FY 95 } \\
\text { Actual }\end{array}$ & $\begin{array}{l}\text { FY } 96 \\
\text { Pres. }\end{array}$ & $\begin{array}{l}\text { FY } 96 \\
\text { Est. }\end{array}$ & $\begin{array}{l}\text { FY } 97 \\
\text { Rast. }\end{array}$ & HECC & LSN & HCS & HuCS & ETHR & HECC & LSN & HCS & HuCS & ETHR \\
\hline $\begin{array}{l}\text { Development and Dissemination of Scientific Software } \\
\text { for HPCS }\end{array}$ & 3.37 & 3.60 & 3.37 & 3.37 & 2.77 & 0.60 & & & & 2.37 & 0.50 & & 0.50 & \\
\hline Infrastructure for Information Technology & 9.48 & 18.24 & 9.48 & 9.48 & 1.22 & 1.86 & 4.20 & 2.20 & & 1.22 & 1.20 & 4.20 & 2.86 & \\
\hline Infrastructure for Information Technology (continued) & & & & & & & & & & & & & & \\
\hline Systems Integration for Manufacturing Applications & 10.66 & 12.26 & 10.66 & 10.66 & 1.80 & 0.50 & 1.00 & 7.36 & & 2.00 & 0.50 & 1.16 & 7.00 & \\
\hline Totals: & 23.51 & 34.10 & 23.51 & 23.51 & 5.79 & 2.96 & 5.20 & 9.56 & & 5.59 & 2.20 & 5.36 & 10.36 & \\
\hline
\end{tabular}




\section{NIST \\ Development and Dissemination of Scientific Software for HPCS \\ Budget Code: \\ STRS for Computer Systems}

This program activity focuses on the development of advanced mathematical, computational and visualization algorithms, software, methodology and tools which support the efficient application of computationally intensive science to key problems arising in the industrial sector. Current application areas of emphasis include the development of improved methods for computational chemistry and related advanced materials processing industries which are important for advanced product and process design and computationally derived fundamental data for competitive, environmentally sound manufacturing processes. Technology areas of emphasis include the interactive visualization of complex structures and data, large-scale ab initio computational chemistry, Monte Carlo and molecular dynamics modeling and scientific database utilization. Includes the development of efficient, robust and flexible templates, class libraries and components for basic mathematical computation, such as the solution of large linear systems, which provide a foundation for applications such as these.

This program activity also supports the development of modern, network-based reusable software classification and distribution technology for making new computational software readily available to industry and the public. The focus of this activity is the Guide to Available Mathematical Software (GAMS) project. GAMS is a working cross index and virtual repository which provides users transparent access to thousands of reusable software modules accessible from a variety of network-accessible repositories.

\section{Milestone}

Changes

$$
\text { FY } 1995 \text { Actual Milestones }
$$

Mixed quantum chemistry/molecular dynamics capability incorporated into GAMESS computer code (Iowa State U. using NIST technology). Capabilities for studying complex systems include both discreet and continuum solvation coupled to state of the art quantum chemistry.

Released Parallel Applications Development Environment v1.0 and 1.2 to facilitate development of PVM codes by applications experts with minimum of PVM

Established Web-accessible data base of critically evaluated data for density-functional electronic structure calculations.

Web access to GAMS of 85,000 users and 800,000 server transactions. Developed Web access for the ACM Transactions on Mathematical Software.

Completed design of suite of packages for high performance object-oriented sparse linear algebra. Released prototype implementations. Initiated

GAMS experiments with free text search engines and $\vec{u}$ improved classification schemes.
FY 1996 Estimated Milestones

Develop and apply algorithms for molecular dynamic simulations of cluster growth and deposition of use in modeling sintering

Develop scalable algorithms for treatment of dissipative systems and apply to optically-trapped atoms and to properties of newly-discovered Bose-Einstein condensates.

Improve search and browsing mechanisms and expand information on algorithms and software available from GAMS servers.

Expand implementation of high-performance object-oriented libraries for sparse linear algebra. Utilize modern software design technologies to implement portable and reliable mathematical software to provide significant performance improvement on scalable computing architectures.

\section{and and}

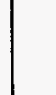

\section{FY 1997 Agency Request Milestones}

Develop a user-friendly code for calculating rate constants for unimolecular decomposition and associated processes.

Develop and implement $a b$ initio quantum mechanical methods, including estimates of accuracy limits, to provide anharmonic corrections to low frequency blends in complex molecules.

Demonstrate the use of this data to improve the accuracy of the calculation of kinetic and thermodynamic parameters.

Develop practical conjugate gradient

algorithm for calculation of electronic structure of metallic systems.

Provide an expanded GAMS service, includin new classification technology that provides expert level performance.

Demonstrate high-performance object-oriented libraries for sparse linear algebra on challenging applications.

Stabilize and release a scalable parallel multigrid solver for unstructured adaptive grids.

\section{Budget (\$ M)}

\begin{tabular}{|c|c|}
\hline FY 95 Act & 3.37 \\
\hline FY 96 Pres. & 3.60 \\
\hline FY 96 Est. & 3.37 \\
\hline FY 97 Rqst. & 3.37 \\
\hline
\end{tabular}

Program

Component

Areas

FY 96 FY 97

\begin{tabular}{|l|l|l|}
\hline HECC & 2.37 & 2.77 \\
\hline LSN & 0.50 & 0.60 \\
\hline HCS & & \\
\hline HuCS & 0.50 & \\
\hline ETHR & & \\
\hline
\end{tabular}

\section{Agency Ties}

\begin{tabular}{|c|c|}
\hline DARPA & \\
\hline NSF & Partner \\
\hline DOE & Partner \\
\hline NASA & Partner \\
\hline NIH & \\
\hline NSA & \\
\hline NIST & \\
\hline NOAA & \\
\hline EPA & User \\
\hline ED & \\
\hline AHCPR & \\
\hline VA & \\
\hline
\end{tabular}

December 20, 1996 
$\rightarrow$ Collaborate with industry and other agencies to support development of standards to provide interoperability, common user interfaces and enhanced

$\overrightarrow{\mathrm{u}}$ security for computer and communications systems; and promotes the specification, development, testing and deployment of communications protocols and

a services within the National Information Infrastructure. Collaborate with industry and academia to conduct R\&D, evaluate and apply methods to specify, verify, and test for conformity and interoperability of high-integrity, distributed systems. Support and contribute to the development of test methods and tes suites for assessing conformity of products to standards and interoperation with other products; develop prototype implementations; establish testbeds and support technology demonstrations; host workshops and open fora to promote open standards and to aid in the commercialization and deployment of information technology by U.S. Industry.

Promote the research, development and application of measurement sciences to assess the performance of human-machine interface technologies, and to assess the performance of high-performance computing and communications systems. Conduct collaborative research and development of algorithms, recognition methods, and reference materials to assist industrial and academic researchers commercialize R\&D in spoken natural language recognition, image recognition including printed or cursive handwritten text, and text search and information retrieval. Collaborate by developing corpora of reference materials for speech and image recognition, and for text search and retrieval; and by developing related scoring protocols which use the reference materials in assessing performance of systems implementing these human-machine interface technologies. Develop methods for measuring performance of scalable, high performance systems, and identification of performance bottlenecks in systems and software.

Collaborate with industry and other agencies to develop, integrate and apply technologies which enable electronic commerce and integration of product specifications into computer aided design and manufacturing tools. Establish laboratory facilities to support demonstrations, evaluation, and inter-operation testing. (continued)

Milestone This element reflects the integration of three elements from the FY96 plan: Networking and Information Infrastructure Services, Metrology for Changes Human-Machine Interfaces \& Scalable HPCS, and Electronic commerce and Digital Libraries. Milestones deferred for one or more years

\section{FY 1995 Actual Milestones \\ Distributed ATM Network Simulator code to}

over 100 academic and industrial sites. Collaborated with ATM Forum and industry to develop standardized test procedures for conformance and interoperability testing -- seven protocol test suites approved by Forum. Created framework for benchmarking of wireless systems and investigated use of automated speech recognition as measure of quality of speech recognition over noisy channels. Established cooperative agreement with $U$. of $M D$ f wireless transmission of multimedia.

Enhanced NIST spoken-language library interface system with a thesaurus built from the Library of Congress subject authority database. Completed initial version of Z39.50/PRISE, a basic Z39.50 UNIX client/ server integrated with the PRISE prototype indexing and search engine.

Demonstrated first public prototype of a scalable tuning tool for parallel software based on statistical techniques.

(continued)

\section{Establish an interoperability testing program} cluding laboratory facilities for Internet security technologies (e.g., firewalls, authentication

mechanisms), IPv.6, network management and NI Services. Assess the security requirements and vulnerabilities of emerging wireless technologies. Develop and test methods for human-machine spoken dialogue management and clarification,

dialogue error recovery, and evaluation of speech corpora portability. Design and implement new architecture for Z39.50/PRISE that will handle complex objects in both search and display modes. Develop initial algorithms for understanding of documents and their component parts, and

associated metrics for judging algorithm performance.

Improve and extend the software and hardware based tools for performance measurement of scalable parallel systems.

Initiate development of conversion tools for electronic data interchange standards. FY 1997 Agency Request Milestones

Develop a user-friendly code for calculating rate constants for unimolecular decomposition and associated processes.

Develop and implement $a b$ initio quantum mechanical methods, including estimates of accuracy limits, to provide anharmonic corrections to low frequency blends in complex molecules.

Demonstrate the use of this data to improve the accuracy of the calculation of kinetic and thermodynamic parameters.

Develop practical conjugate gradient

algorithm for calculation of electronic structure of metallic systems.

Provide an expanded GAMS service, including new classification technology that provides expert level performance.

Demonstrate high-performance object-oriente libraries for sparse linear algebra on challenging anplications.

Stabilize and release a scalable parallel multigrid solver for unstructured adaptive grids.

\section{Budget (\$ M)}

\begin{tabular}{|c|r|}
\hline FY 95 Act & 9.48 \\
\hline FY 96 Pres. & 18.24 \\
\hline FY 96 Est. & 9.48 \\
\hline FY 97 Rqst. & 9.48 \\
\hline
\end{tabular}

Program

Component

Areas

FY $9 6 \longdiv { \text { FY } 9 7 }$

\begin{tabular}{|c|c|c|}
\hline HECC & 1.22 & 1.22 \\
\hline LSN & 1.20 & 1.86 \\
\hline HCS & 4.20 & 4.20 \\
\hline HuCS & 2.86 & 2.20 \\
\hline ETHR & & \\
\hline
\end{tabular}

Agency Ties

\begin{tabular}{|c|c|}
\hline DARPA & Partner \\
\hline NSF & Partner \\
\hline DOE & Partner \\
\hline NASA & User \\
\hline NIH & User \\
\hline NSA & Partner \\
\hline NIST & \\
\hline NOAA & User \\
\hline EPA & User \\
\hline ED & User \\
\hline AHCPR & User \\
\hline VA & Partner \\
\hline
\end{tabular}

December 20, 1996 


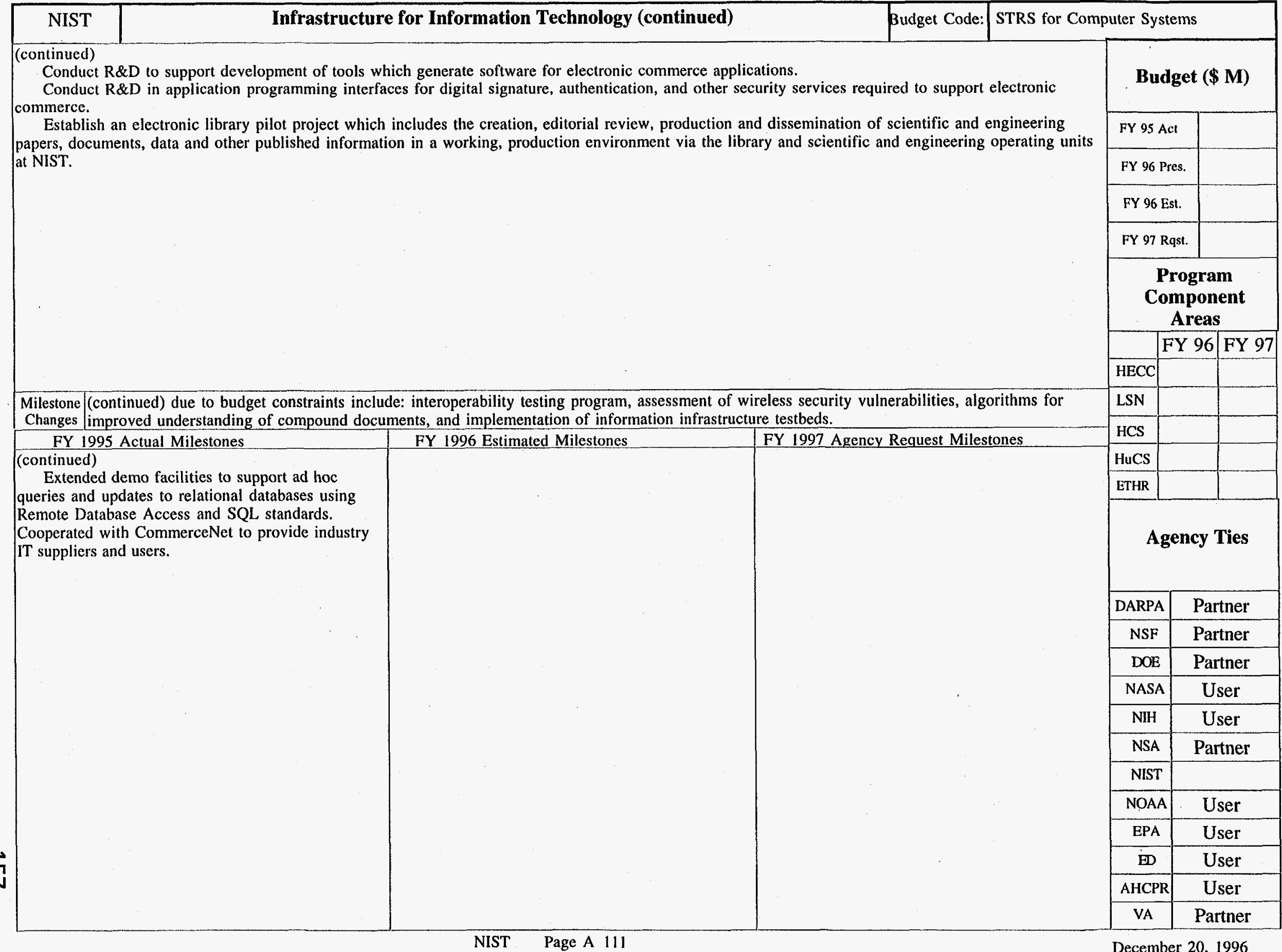


Emphasis is on information technology solutions providing reliable information exchange among manufacturing applications. This standards-based effort for computer integrated manufacturing focuses on integration problems existing in the activities of product/process design, manufacturing engineering, and production system control. The effort will address these problem areas both within and across enterprises. Manufacturing application integration mechanisms will be developed and structured in the context of emerging Enterprise Integration Frameworks (EIFs). Integration mechanisms supporting incorporation of remote data sources into manufacturing applications as well as distributing manufacturing software application components and processes will be a significant focus. Development of reliable mechanisms for testing of manufacturing application integration solutions is also a priority. The program of work addresses activities which fall within standards development efforts and development of technology within industry and government. Interface specifications which have been tested in prototype systems will be communicated to standards organizations. Results will be made available to U.S. industry through workshops, training materials, electronic data repositories, and pre-commercial prototype implementations that can be installed for test and evaluation. NIST will distribute standard reference data, technical information, and digital product/process specifications using NII technologies. The industry domains to be addressed include discrete mechanical product manufacturing, electronic manufacturing, chemical manufacturing, material manufacturing, and manufacturing plant construction. NIST's Advanced Manufacturing and Networking Testbed (AMSANT) facilities support application of high performance computing and networking technologies to manufacturing system and application integration problems. Facilities include Asynchronous Transfer Mode (ATM) network connections, high performance compute servers and workstations, as well as software applications for mechanical computer-aided design (CAD), electronic CAD, architectural/engineering/construction $C A D$, product data management, process planning, production system scheduling, machine tool programming, and process simulation. Hardware and software supporting application of collaborative technologies, remote operation, virtual reality interfaces/environments to the manufacturing domain is also employed. The facilities serve as development, testing, and demonstration sites for use by NIST, government partners, and industrial technology suppliers and users. The program of work strongly supports cooperative research and development agreements with industry and academic researchers.

Milestone Moved to FY 97: Enterprise Integration Framework deliverables;

Changes Implementation of a production testbed for Custom Therapeutic Footwear; and

\section{FY 1995 Actual Milestones}

Issued: report on manufacturing integration needs a requirements document on an integrated engineering tools environment, a reference architecture for control of mechanical systems, and a reference model architecture for design, planning and production activities.

Demonstrated selected set of conformance testing capabilitics for STEP and implemented a testing program for Application Protocols.

Implemented operational HPCC testbed for

mechanical parts industries and completed a series

of remote tests with DOE partner and a visualization

lab to test virtual reality applications in

manufacturing.

Established laboratory to test and demonstrate a standard set of user interfaces for manufacturing production systems.

Tested a series of process models from industry defined product realization processes (PRP), and developed a common set of PRP models for manufacturing resources.

Made scientific, engineering, and standards reference data available and accessible over the web.

\section{FY 1996 Estimated Milestones}

Demonstrate a selected set of interface

protocols and integration capabilities for the design planning and production of mechanical production.

Publish a series of interface specification

documents for integrating manufacturing software applications.

Extend AMSANT capabilities and perform a series of remote integration tests between NIST, Industry and DOE partner HPCC testbed.

Develop level II APDE capabilities and deploy system to base set of user within standards community.

Publish a report on the engineering architecture for integrating design, planning and production applications.

Expand scope of manufacturing research and standard reference data available through the web.
FY 1997 Agency Request Milestones

Conduct a series of workshops on Enterprise integration Frameworks (EIF), publish a report for mplementing EIF, and extend AMSANT facilitie to test and demonstrate EIF tools and products. Demonstrate an extended set of interface protocols and integration capabilities for the design, planning and production of mechanical systems.

Publish an extended series of interface specification documents for integrating manufacturing software applications.

Enhance APDE capabilities and establish metrics for tracking use of STEP and progress towards its development.

Deploy industrial pilot of implementation of SIMA specifications and standards based on implementation architecture.

\section{Budget (\$ M)}

\begin{tabular}{|l|l|}
\hline FY 95 Act & 10.66 \\
\hline FY 96 Pres. & 12.26 \\
\hline FY 96 Est. & 10.66 \\
\hline FY 97 Rqst. & 10.66 \\
\hline
\end{tabular}

Program

Component

Areas

\begin{tabular}{|l|l|} 
FY 96 & FY 97 \\
\hline
\end{tabular}

\begin{tabular}{|l|l|l|}
\hline HECC & 2.00 & 1.80 \\
\hline
\end{tabular}

LSN

HCS

0.50

1.16

HuCS

ETHR

7.00

1.00

Agency Ties

\begin{tabular}{|c|c|}
\hline DARPA & Partner \\
\hline NSF & \\
\hline DOE & Partner \\
\hline NASA & \\
\hline NIH & \\
\hline NSA & \\
\hline NIST & \\
\hline NOAA & \\
\hline EPA & \\
\hline ED & \\
\hline AHCPR & \\
\hline VA & \\
\hline
\end{tabular}

December 20, 1996 


\section{National Oceanic and Atmospheric Administration}

NOAA's Grand Challenge research in weather forecasting and climate prediction depends on advances in high-end computing and on the collection and dissemination of environmental information. Increased computing power will enable more accurate representation of the atmosphere-ocean system, resulting in improved weather forecasts and making possible better decision making by Government and industry on issues that affect both the environment and the economy. NOAA's environmental data and information on the NII will be enabled through NOAA's ability to disseminate its vast holdings of real-time and historical information to all users more completely, in a more usable form, and in a much more timely manner.

\begin{tabular}{|c|c|c|c|c|c|c|c|c|c|c|c|}
\hline \multirow{2}{*}{ Program Activity } & \multirow[b]{2}{*}{ Budget Account Code } & \multirow{2}{*}{$\begin{array}{c}\text { Partner/User } \\
\text { Agencies }\end{array}$} & \multicolumn{4}{|c|}{ Budget $(\mathrm{BA}, \$ \mathrm{M})$} & \multicolumn{5}{|c|}{ HPCCPCAs by 1997 Pres. Request } \\
\hline & & & $\begin{array}{l}\text { FY 95 } \\
\text { Actual }\end{array}$ & $\begin{array}{c}\text { FY } 96 \\
\text { Pres. }\end{array}$ & $\begin{array}{c}\text { FY } 96 \\
\text { Est. }\end{array}$ & $\begin{array}{l}\text { FY } 97 \\
\text { Rqst. }\end{array}$ & HECC & LSN & HCS & HuCS & ETHR \\
\hline Advanced Computation & ORF EI & $\begin{array}{l}\text { ARPA, NSF, } \\
\text { DOE }\end{array}$ & 2.80 & 7.40 & 3.30 & 6.30 & 6.30 & & & & \\
\hline Networking Connectivity & ORF EI & NASA & 2.70 & 7.50 & 2.70 & 2.70 & & 2.70 & & & \\
\hline Information Dissemination Pilots & ORFEI & & & 0.50 & 0.50 & 0.50 & & & & 0.50 & \\
\hline & & Totals: & 5.50 & 15.40 & 6.50 & 9.50 & 6.30 & 2.70 & & 0.50 & \\
\hline
\end{tabular}


National Oceanic and Atmospheric Administration

$\vec{\sigma}$

\begin{tabular}{|c|c|c|c|c|c|c|c|c|c|c|c|c|c|c|c|}
\hline \multirow[b]{2}{*}{ Program Activity } & & \multicolumn{4}{|c|}{ Budget (BA, \$ M) } & \multicolumn{5}{|c|}{ HPCC PCAs by 1997 Pres. Request } & \multicolumn{5}{|c|}{ HPCC PCAs by 1996 Estimated } \\
\hline & & \begin{tabular}{l|} 
FY 95 \\
Actual
\end{tabular} & $\begin{array}{l}\text { FY } 96 \\
\text { Pres. }\end{array}$ & $\begin{array}{c}\text { FY } 96 \\
\text { Est. }\end{array}$ & $\begin{array}{l}\text { FY } 97 \\
\text { Rast. }\end{array}$ & HECC & LSN & HCS & HuCS & ETHR & HECC & LSN & HCS & HuCS & ETHR \\
\hline Advanced Computation & & 2.80 & 7.40 & 3.30 & 6.30 & 6.30 & & & & & 3.30 & & & & \\
\hline Networking Connectivity & & 2.70 & 7.50 & 2.70 & 2.70 & & 2.70 & & & & & 2.70 & & & \\
\hline Information Dissemination Pilots & & & 0.50 & 0.50 & 0.50 & & & & 0.50 & & & & & 0.50 & \\
\hline & Totals: & 5.50 & 15.40 & 6.50 & 9.50 & 6.30 & 2.70 & & 0.50 & & 3.30 & 2.70 & & 0.50 & \\
\hline
\end{tabular}




\section{NOAA}

Advanced Computation

\begin{tabular}{|l|l|l} 
Budget Code: & ORF EI \\
\hline
\end{tabular}

Advanced Scalable Computation is NOAA's program to make possible major improvement in the Nation's ability to forecast the weather and predict climate change by taking full advantage of highly parallel, high performance computing systems that, over the long term, are expected to provide substantially greater computing power at lower cost.

Geophysical Fluid Dynamics Laboratory.: NOAA/GFDL's collaborative efforts with DOE/LANL have resulted in the scientific implementation of a parallel version of a very high-resolution global atmospheric grid-point model in a study of stratospheric dynamics on a scalable system.

National Meteorlogical Center (NMC): NOAA/NMC's collaborative efforts with DOD/NRL have resulted in execution of a parallel, adiabatic version of the NMC global spectral model with excellent performance at high resolution.

Forecast Systems Laboratory (FSL): The Intel Paragon installed at NOAA/FSL is being used by NOAA scientists in conjunction with the

DARPA-sponsored National Consortium for High-Performance Computing and as a part of the Boulder Front Range Consortium involving NCAR, the

University of Colorado, and FSL.

NOAA Northwest Center's collaboration efforts through the Cooperative Institute for Arctic Research (CIFAR) is a cooperative government-university-industry effort in environmental research utilizing the Alaska Regional Supercomputer Center.

\begin{tabular}{|l|l} 
Milestone & Scalable HPCS for NOAA/NMC changed from FY 96 to FY 97
\end{tabular}

Changes Implementation of an advanced visualization laboratory to support high-end climate and weather research postponed from FY 96 to a later date.

FY 1995 Actual Milestones support major improvements in climate and weather research. Completed high-resolution stratospheric dynamics experiment
LANL scalable HPCS using a parallel version of the SKYHI on LANL scalable HPCS using a par
global atmospheric grid-point model.

global atmospheric grid-point model.
Initiated preliminary 3-D cloud-radiation interaction

Initiated preliminary 3-D cloud-radiation interaction
experiments using the redesigned parallel GFDL limited-area experiments using the
non-hydrostatic model.

Developed and executed parallel version of NMC global
Dydrostatic model.

adiabatic spectral model on a scalable computer, resulting in exceptional wall-clock performance.

Developed a parallel version of finite-difference global ETA model including a new formulation of global grid mesh.

Developed parallel structure for NMC Optimum Interpolation

data assimilation code.

Completed preliminary implementation of the restructured

ETA model on FSL's Intel Paragon and began extensive testing to

evaluate its performance in terms of resolution and the potential

impact for improved forecast guidance. Implemented parallel

versions of the Rapid Update Cycle (RUC) and the Regional

Atmospheric Modeling System (RAMS) to run, for the first time,

in real time on the Intel Paragon.

$\vec{\sigma}$

\section{FY 1996 Estimated Milestones} Implement scientific experiments utilizing redesigned Calable weather and climate models on HPCS at NOAA/GFDL. Complete development of scalable versions of NMC global spectral and ETA models for scalable architectures and compare
performance on different scalable HPCSs. Perform ensemble performast experiments on scalable HPCS.

Complete, test and run in real time the restructured ETA

model $(8-16 \mathrm{~km}$ resolution) on a scalable hardware machine prototype forecast experiment in support of the 1996 Olympic Games.

Develop and test restructured parallel version of regional data similation code.

Implement ETA model on FSL's Intel Paragon; evaluate performance at various grid resolutions and assess potential for operational forecast purposes. Update real-time experimental forecast models (RUC and RAMS) to support nesting.
FY 1997 Agency Request Milestones Procure a scalable HPCS for NOAANMC to advance future improvements in operational weather forecasting for the ation.

Initiate algorithm development on scalable system toward chieving $5-10 \mathrm{~km}$ resolution in mesoscale atmospheric models.

Explore design of next-generation environmental observing systems utilizing HPCS to test data assimilation needs for ptimizing future forecast systems.

Develop software tools to facilitate software conversion om traditional shared-memory machine to scalable systems. Continue and enhance scientific experiments running on TPCS at NOAA/GFDL.

Implement ETA model on FSL's Intel Paragon; evaluate performance at various grid resolutions and assess potential for operational forecast purposes. Update real-time experimenta orecast models (RUC and RAMS) to support nesting. Implement advanced visualization laboratory to support high-end climate and weather research.
Budget (\$ M)

\begin{tabular}{|c|c|}
\hline FY 95 Act & 2.80 \\
\hline FY 96 Pres. & 7.40 \\
\hline FY 96 Est. & 3.30 \\
\hline FY 97 Rqst. & 6.30 \\
\hline
\end{tabular}

Program

Component

Areas

FY 96 FY 97

\begin{tabular}{|l|l|l|}
\hline HECC & 3.30 & 6.30 \\
\hline LSN & & \\
\hline HCS & & \\
\hline HuCS & & \\
\hline ETHR & & \\
\hline
\end{tabular}

Agency Ties

\begin{tabular}{|c|c|}
\hline DARPA & Partner \\
\hline NSF & Partner \\
\hline DOE & Partner \\
\hline NASA & \\
\hline NIH & \\
\hline NSA & \\
\hline NIST & \\
\hline NOAA & \\
\hline EPA & \\
\hline ED & \\
\hline AHCPR & \\
\hline VA & \\
\hline
\end{tabular}

December 20, 1996 


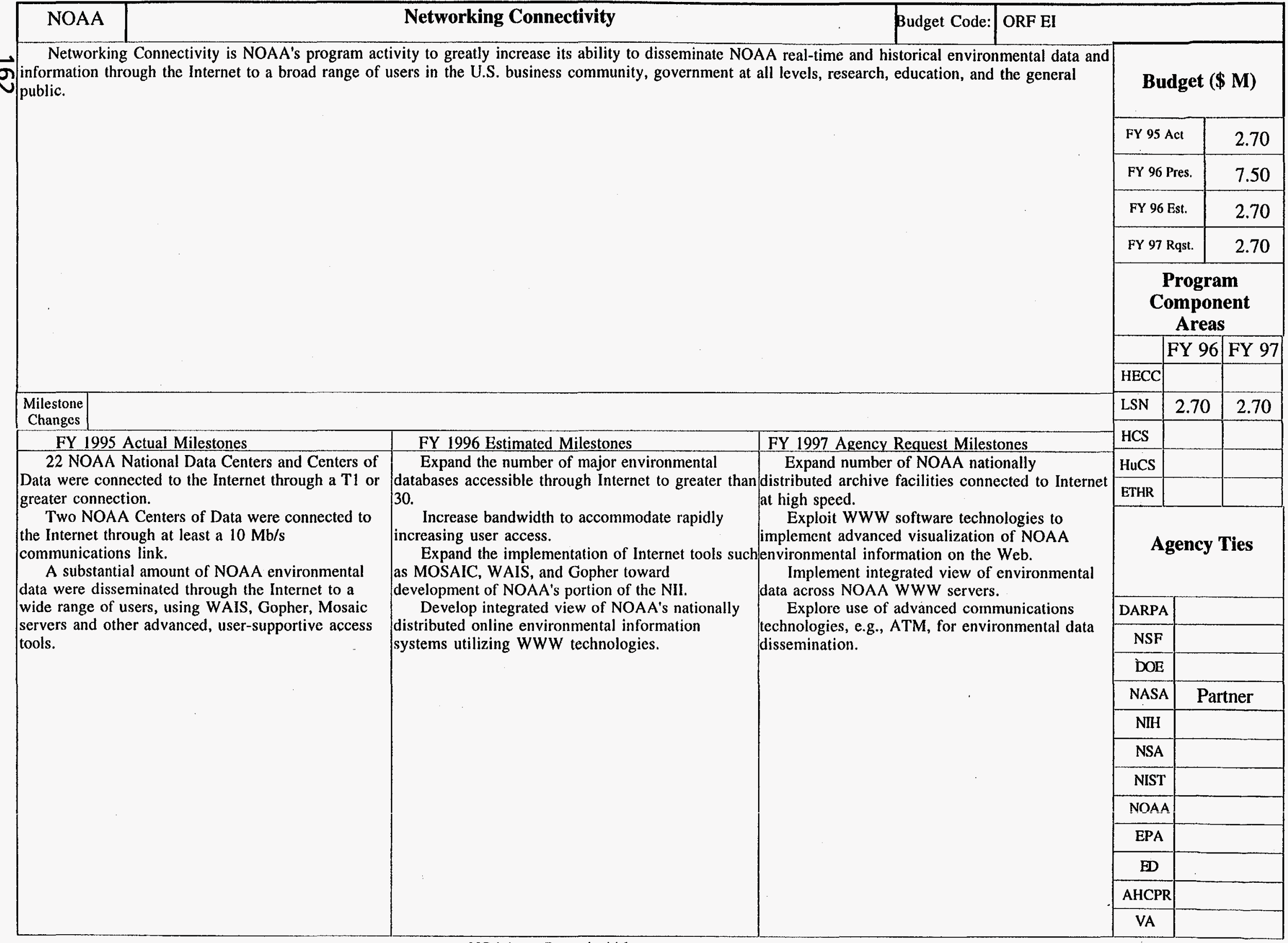




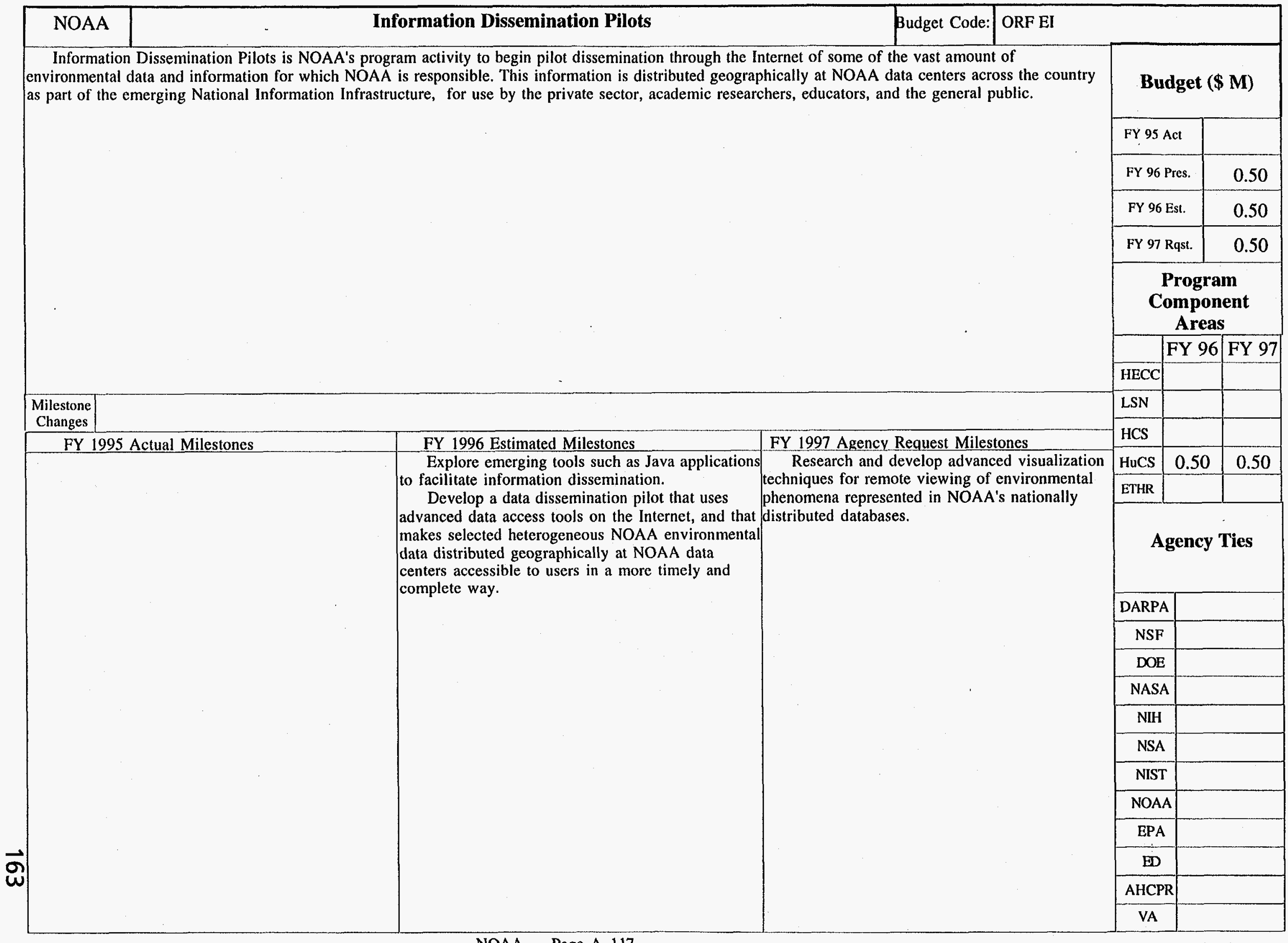




\section{Environmental Protection Agency}

$\vec{\sigma}$ The goal of the EPA's HPCC program is to accelerate the evolution of high-end computing technologies and their use to meet environmental Protection mission objectives. HPCC technology enables more effective, scientifically defensible, and timely environmental decision making resulting in economic benefits for states and industry by reducing the cost of achieving legislated environmental standards and by increasing the market for HPCC technologies. Research focuses on high-end computing technologies required to facilitate multi-pollutant, multi-scale, cross media environmental modeling, risk assessments, and community decision making.

The research targets technologies for advanced problem solving environments, reusable software needed for rapid intelligent data access and synthesis, integrated visualization and geographical information systems capabilities closely tied to environmental modeling, and scalable parallel computing and communications to support multi-disciplinary ecosystem risk assessments. Research in distributed computing provides for better access and management of large collections of environmental data and sharing of powerful computational resources needed to support advanced environmental problem solving.

To facilitate technology transfer of high-end environmental assessment tools to real world users, the EPA HPCC program is involved in a series of pilot projects. These projects provide for open communication of the needs of environmental scientists, analysts and decision makers.

\begin{tabular}{|c|c|c|c|c|c|c|c|c|c|c|c|}
\hline \multirow[b]{2}{*}{ Program Activity } & \multirow[b]{2}{*}{ Budget Account Code } & \multirow{2}{*}{$\begin{array}{l}\text { Partner/User } \\
\text { Agencies }\end{array}$} & \multicolumn{4}{|c|}{ Budget (BA, $\$ M$ ) } & \multicolumn{5}{|c|}{ HPCCPCAs by 1997 Pres. Request } \\
\hline & & & $\begin{array}{l}\text { FY 95 } \\
\text { Actual }\end{array}$ & $\begin{array}{c}\text { FY 96 } \\
\text { Pres. }\end{array}$ & $\begin{array}{l}\text { FY } 96 \\
\text { Est. }\end{array}$ & $\begin{array}{c}\text { FY } 97 \\
\text { Rqst. }\end{array}$ & HECC & LSN & HCS & HuCS & ETHR \\
\hline Environmental Modeling & $\mathrm{CC} 9, \mathrm{CCl}$ & NSF & 5.65 & 5.78 & 5.53 & 3.45 & 3.45 & & & & \\
\hline Computational Techniques & $\mathrm{CC} 9, \mathrm{CCl}, \mathrm{CA} 2$ & DOE & 5.10 & 3.50 & 3.17 & 3.13 & 3.13 & & & & \\
\hline State Network Connectivity & $\mathrm{CC} 9$ & & 0.69 & 0.69 & & & & & & & \\
\hline Education/Training & $\mathrm{CC} 9$ & & 0.69 & 1.01 & 0.08 & & & & & & \\
\hline Public Data Access & $\mathrm{CC} 9$ & NASA, NOAA & 0.30 & 1.02 & 0.60 & 0.60 & & & & 0.60 & \\
\hline & & Totals: & 12.43 & 12.00 & 9.38 & 7.18 & 6.58 & & & 0.60 & \\
\hline
\end{tabular}




\section{Environmental Protection Agency}

\begin{tabular}{|c|c|c|c|c|c|c|c|c|c|c|c|c|c|c|c|}
\hline \multirow[b]{2}{*}{ Program Activity } & & \multicolumn{4}{|c|}{ Budget (BA, \$ M) } & \multicolumn{5}{|c|}{ HPCC PCAs by 1997 Pres. Request } & \multicolumn{5}{|c|}{ HPCC PCAs by 1996 Estimated } \\
\hline & & $\begin{array}{l}\text { FY } 95 \\
\text { Actual }\end{array}$ & $\begin{array}{c}\text { FY } 96 \\
\text { Pres. }\end{array}$ & $\begin{array}{c}\text { FY } 96 \\
\text { Est. }\end{array}$ & $\begin{array}{l}\text { FY } 97 \\
\text { Rast. }\end{array}$ & HECC & LSN & HCS & HuCS & ETHR & HECC & LSN & HCS & HuCS & ETHR \\
\hline Environmental Modeling & & 5.65 & 5.78 & 5.53 & 3.45 & 3.45 & & & & & 5.53 & & & & \\
\hline Computational Techniques & & 5.10 & 3.50 & 3.17 & 3.13 & 3.13 & & & & & 3.17 & & & & \\
\hline State Network Connectivity & & 0.69 & 0.69 & & & & & & & & & & & & \\
\hline Education/Training & & 0.69 & 1.01 & 0.08 & & & & & & & & & & & 0.08 \\
\hline Public Data Access & & 0.30 & 1.02 & 0.60 & 0.60 & & & & 0.60 & & & & & 0.60 & \\
\hline & Totals: & 12.43 & 12.00 & 9.38 & 7.18 & 6.58 & & & 0.60 & & 8.70 & & & 0.60 & 0.08 \\
\hline
\end{tabular}


This program activity supports fundamental research on the systematic integration of advanced multi-pollutant, multi-scale, and multi-media न environmental modeling components into a high performance distributed computing framework, addressing such issues as human-computer interface, O) distributed data management, software reuse and scalability, and system performance.

EPA is integrating research results to develop a technology foundation to support community-based environmental decision-making and risk assessment. The objective is a community multi-discipline problem solving framework for environmental modeling and decision support built upon emerging HPCC

technology and capable of adapting to continuous advances in science and technology. The initial focus of the program was on oxidant, acid deposition, and particulate models, but has been expanding to encompass integration of more complex processes such as aerosols and visibility, and cross-media ecosystem assessments. Both research and prototyping of system framework capabilities such as graphical user interface, intelligent system builders and data management, collaborative tools, interactive analysis and visualization, multimedia electronic 'tutor and help', and decision support are being performed to better define technology/user requirements and design alternatives for environmental modeling and decision support systems. The resultant modeling and decision support framework will have general applicability for use in air quality, surface and ground water quality modeling and ecosystem management. Advanced collaborative methods of analyzing and visualizing the multi-dimensional measurements and model predictions from environmental assessment studies are being developed to provide a means of gaining greater insight into interactions of the science in the models and for better interpretation of results.

\section{Milestone The reduction in the EPA FY 96 HPCC budget delayed linkage of air and water models.}

\section{Changes}

\section{FY 1995 Actual Milestones}

Demonstrated a prototype modular,scalable, air quality modeling decision support system in a distributed computing environment.

Developed an interactive environment to

facilitate the calibration, execution, and analysis of the Chesapeake Bay Watershed model for nitrogen eutrophication studies.

Developed collaborative computing and visualization prototype for several air quality applications to enable distant researchers to co-examine data and effectively use remote visualization resources.

\section{FY 1996 Estimated Milestones}

Develop an operational air quality modeling and decision support system for regional and urban oxidant, regional acid deposition, and particulate issues.

Prototype a modular, scalable air quality modeling decision support system with integrated sensitivity-uncertainty analysis.

Implement video conferencing to key agency and research sites.

Award grants for research to advance environmental decision support tools through coupling of Geographical Information Systems and environmental models, parallel and distributd optimization techniques, and advanced multivariate visualization techniques.

\section{FY 1997 Agency Request Milestones}

Implement linked air and water quality model in a distributed computing environment to

facilitate cross-media assessment of water quality Continue grants for research to advance environmental decision support tools through coupling of Geographic Information Systems and environmental models, parallel and distributed optimization techniques, and advanced multivariate visualization techniques.
Budget (\$ M)

\begin{tabular}{|c|c|}
\hline FY 95 Act & 5.65 \\
\hline FY 96 Pres. & 5.78 \\
\hline FY 96 Est. & 5.53 \\
\hline FY 97 Rqst. & 3.45 \\
\hline
\end{tabular}

\section{Program}

Component

Areas

FY 96 FY 97

\begin{tabular}{|l|l|l|}
\hline HECC & 5.53 & 3.45 \\
\hline LSN & & \\
\hline HCS & & \\
\hline HuCS & & \\
\hline ETHR & & \\
\hline
\end{tabular}

Agency Ties

\begin{tabular}{|c|c|}
\hline DARPA & \\
\hline NSF & Partner \\
\hline DOE & \\
\hline NASA & \\
\hline NIH & \\
\hline NSA & \\
\hline NIST & \\
\hline NOAA & \\
\hline EPA & \\
\hline ED & \\
\hline AHCPR & \\
\hline VA & \\
\hline
\end{tabular}

December 20, 1996 
The primary objective of the computational techniques research is to develop and evaluate the performance of key algorithms that form the computational foundation of air, water, and molecular models on a variety of scalable parallel architectures. Numerical algorithm research includes grid generation and coordinate transformation techniques applicable to multiscale air quality modeling, comparative study of parallel programming models for gas-phase atmospheric chemistry solvers, parallel algorithm development and evaluation for several air and water quality, ground water, stream and lake sedimentation, meteorology, and molecular modeling processes. Fundamental research is also conducted on computational techniques for quantifying uncertainty as an integral part of the numerical computation.

The research evaluates combinations of hardware, software, algorithms, and communication capabilities to determine the most effective computational approach to support more complex multi- pollutant and cross-media environmental modeling activities. This research, performed at several universities, the DOE's Argonne National Laboratory, and several EPA research laboratories, directly impacts the performance of environmental Grand Challenge applications and contributes to the shared software libraries and parallel testbed codes.

To fully integrate results of the research into the mainstream of EPA computational-based research activities, EPA has acquired a scalable parallel computer.

\section{Milestone $\quad$ Agency budget reductions eliminate planned upgrades for scalable computing.}

\section{Changes}

\section{FY 1995 Actual Milestones}

Developed and implemented parallel codes for several gas-phase atmospheric chemistry solvers to evaluate performance of a variety of parallel architectures.

Implemented portable parallel meteorological testbed model to support advanced air quality models.

Acquired a scalable parallel computing system to $\begin{aligned} & \text { for optimization problems, coupled air and water } \\ & \text { exche processes, and adaptive grid approaches. }\end{aligned}$ support HPCC research activities.

\section{FY 1996 Estimated Milestones}

Develop a parallel analytic element method for ground water modeling.

Implement parallel, distributed, gas-phase

chemistry code for use in operational advanced air quality models. for optimization problems, coupled air and water

\section{FY 1997 Agency Request Milestones}

Incorporate 4-D data assimilation into paralle meteorology models.

Develop parallel algorithms for aerosol namics.

Award grants for development of parallel lgorithms and domain decomposition approaches or multi-media modeling.

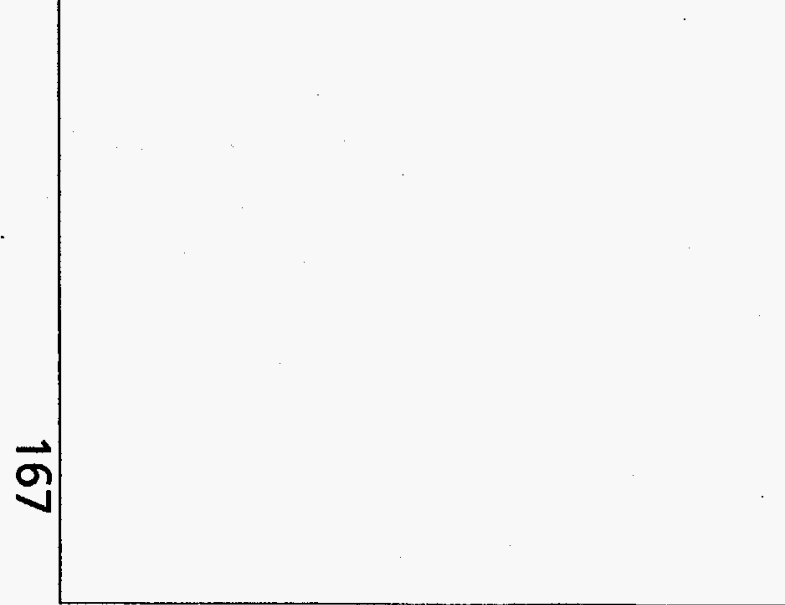

\section{Budget (\$M)}

\begin{tabular}{|l|l|}
\hline FY 95 Act & 5.10 \\
\hline FY 96 Pres. & 3.50 \\
\hline FY 96 Est. & 3.17 \\
\hline FY 97 Rqst. & 3.13 \\
\hline
\end{tabular}

Program

Component

Areas

FY $9 6 \longdiv { F Y ~ } 9 7$

\begin{tabular}{|l|l|l|}
\hline LSN & & \\
\hline HCS & & \\
\hline HuCS & & \\
\hline ETHR & & \\
\hline
\end{tabular}

Agency Ties

\begin{tabular}{|c|c|}
\hline DARPA & \\
\hline NSF & \\
\hline DOE & Partner \\
\hline NASA & \\
\hline NIH & \\
\hline NSA & \\
\hline NIST & \\
\hline NOAA & \\
\hline EPA & \\
\hline ED & \\
\hline AHCPR & \\
\hline VA & \\
\hline
\end{tabular}

December 20, 1996 
The main objectives of this program activity are to provide access to the Internet for a critical mass of key environmental problem-solving groups in State F environmental communities in order to accelerate the wider use of more accurate and reliable environmental tools, and to provide capabilities for testing

$\infty$ distributed computing approaches with complex multi-pollutant and cross-media environmental models. This activity supported the initiation of network connections for four State and several federal pilot projects for evaluation of environmental assessment tools, Grand Challenge team research, and agency access to high performance computing.

This activity also provided high speed (45 million bits per second) interconnection through the Internet to the EPA's National Environmental Supercomputing Center in Bay City, Michigan with dedicated high speed connectivity to the EPA National Computing Center and HPCC research network in North Carolina. This interconnection established a basic foundation for the use of advanced environmental assessment tools throughout the agency and facilitales technology transfer to State environmental groups. Continued support for these critical network connections have been transferred to the program offices and will no longer be supported within the EPA HPCC program.

Milestone Maintenance of established State Internet connections was transferred to other programs.

\section{Changes}

FY 1995 Actual Milestones
Tested the use of ATM technology for
environmental applications.
Maintained agency Internet and State connections.

\section{FY 1996 Estimated Milestones}

FY 1997 Agency Request Milestones

\section{Budget (\$ M)}

\begin{tabular}{|c|c|c|}
\hline \multicolumn{2}{|c|}{ FY 95 Act } & 0.69 \\
\hline \multicolumn{2}{|c|}{ FY 96 Pres. } & 0.69 \\
\hline \multicolumn{3}{|c|}{ FY 96 Est. } \\
\hline \multicolumn{3}{|c|}{ FY 97 Rqst. } \\
\hline \multicolumn{3}{|c|}{$\begin{array}{c}\text { Program } \\
\text { Component } \\
\text { Areas }\end{array}$} \\
\hline & FY 96 & FY 97 \\
\hline HECC & & \\
\hline LSN & & \\
\hline HCS & & \\
\hline $\mathrm{HuCS}$ & & \\
\hline ETHR & & \\
\hline
\end{tabular}

Agency Ties

\begin{tabular}{|c|c|}
\hline DARPA & \\
\hline NSF & \\
\hline DOE & \\
\hline NASA & \\
\hline NIH & \\
\hline NSA & \\
\hline NIST & \\
\hline NOAA & \\
\hline EPA & \\
\hline ED & \\
\hline AHCPR & \\
\hline VA & \\
\hline
\end{tabular}

December 20, 1996 
This program activity targets the dissemination of high performance computing and advanced environmental modeling capabilities to select groups from industry, State and federal sectors. The industrial group is represented by a consortium, the Consortium for Advanced Modeling of Regional Air Quality. The targel groups of State users are selected from States with professionals involved in front-line environmental decision making and using models of regional and urban air quality. Pilot users gain first-hand experience on how high performance computing can alleviate many of their decision making constraints while at the same time they work with developers to ensure that system capabilities and interfaces address their needs. A high performance computing learning environment is created through workshops, involvement of professional trainers and curriculum developers, and establishment of a 'virtual center' which functions remotely through Internet to support a distributed base of users of environmental models for decision making across the country.

Funding also supported EPA's EarthVision computational science educational program for high school students, graduate students, training fellowships, conferences and workshops.

\section{Milestone Budget reductions eliminated planned environmental education grants}

Changes

\section{FY 1995 Actual Milestones}

Prototyped technology transfer methods for

advanced air quality modeling systems and defined concept for a distributed user/modeling support "center".

Conducted workshops to gather feedback from early use of prototype environmental decision suppor tools.

\section{FY} advanced modeling and training to a broad user community.

\section{FY 1997 Agency Request Milestone

97 Agency Request Milestones

FY




\begin{tabular}{|c|c|c|c|c|c|c|}
\hline EPA & Public Data Access & Budget Code: & $\mathrm{CC} 9$ & & & \\
\hline \multirow{8}{*}{\multicolumn{4}{|c|}{$\begin{array}{l}\text { A wealth of environmental data from diverse sources at multiple scales exists and continues to accumulate each day. Scientists, policy makers, industry } \\
\text { and the general public have diverse needs for data access, manipulation, summarization, and interpretation. This research addresses fundamental technology } \\
\text { issues associated with a multidiscipline approach to finding, assimilating, and analyzing useful information from large, distributed multi-media } \\
\text { environmental databases. Technology areas include intelligent agents for searches among data collections, natural language data navigation techniques, } \\
\text { machine learning, rule-based systems, data visualization, object data bases, interoperability over heterogeneous hardware/software platforms, and synthesis } \\
\text { of information for scientific and public use. }\end{array}$}} & \multicolumn{3}{|c|}{ Budget (\$ M) } \\
\hline & & & & \multicolumn{2}{|c|}{ FY 95 Act } & 0.30 \\
\hline & & & & \multicolumn{2}{|c|}{ FY 96 Pres. } & 1.02 \\
\hline & & & & \multicolumn{2}{|c|}{ FY 96 Est. } & 0.60 \\
\hline & & & & \multicolumn{2}{|c|}{ FY 97 Rqst. } & 0.60 \\
\hline & & & & \multicolumn{3}{|c|}{$\begin{array}{c}\text { Program } \\
\text { Component } \\
\text { Areas } \\
\end{array}$} \\
\hline & & & & \multicolumn{2}{|r|}{ FY 96} & FY 97 \\
\hline & & & & \multicolumn{3}{|l|}{ HECC } \\
\hline \multicolumn{4}{|l|}{\begin{tabular}{|c|} 
Milestone \\
Changes
\end{tabular}} & \multicolumn{3}{|l|}{ LSN } \\
\hline \multirow{16}{*}{ FY 19} & FY 1996 Estimated Milestones & FY 1997 Agency Request Milest & tones & HCS & & \\
\hline & \multirow{15}{*}{$\begin{array}{l}\text { Award grant(s) for development of data } \\
\text { assimilation, and analysis techniques for multi-scale } \\
\text { environmental data including satellite imagery. }\end{array}$} & \multirow{15}{*}{\multicolumn{2}{|c|}{$\begin{array}{l}\text { Continue grant(s) for development of data } \\
\text { assimilation and analysis techniques for } \\
\text { multi-scale environmental data including satellite } \\
\text { imagery. }\end{array}$}} & HuCS & 0.60 & 0.60 \\
\hline & & & & ETHR & & \\
\hline & & & & \multicolumn{3}{|c|}{ Agency Ties } \\
\hline & & & & DARPA & & \\
\hline & & & & NSF & & \\
\hline & & & & DOE & & \\
\hline & & & & NASA & Par & ther \\
\hline & & & & $\mathrm{NIH}$ & & \\
\hline & & & & NSA & & \\
\hline & & & & NIST & & \\
\hline & & & & NOAA & $\mathrm{Pat}$ & ther \\
\hline & & & & $\mathrm{EPA}$ & & \\
\hline & & & & ED & & \\
\hline & & & & AHCPR & & \\
\hline & & & & $\mathrm{VA}$ & & \\
\hline
\end{tabular}




\section{Department of Education}

The U.S. Department of Education (ED) conducts research and development in a number of areas relating to developing resources for and skills within the Education community.

These activities are conducted within the Office of Educational Research and Improvement (OERI), the National Institute for Disabilities Research and rehabilitation (NIDRR) in the Office of Special Education and Rehabilitation Services, and GOALS 2000.

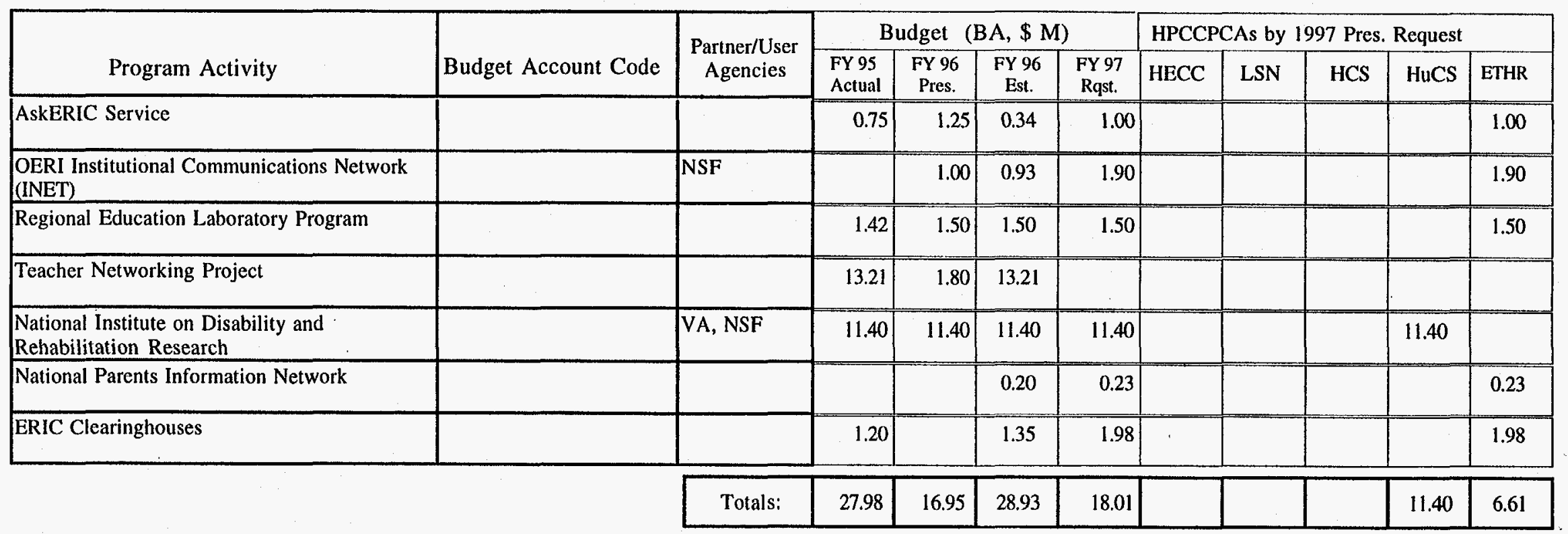




\section{Department of Education}

$\vec{N}$

\begin{tabular}{|c|c|c|c|c|c|c|c|c|c|c|c|c|c|c|}
\hline \multirow[b]{2}{*}{ Program Activity } & \multicolumn{4}{|c|}{ Budget $(\mathrm{BA}, \$ \mathrm{M})$} & \multicolumn{5}{|c|}{ HPCC PCAs by 1997 Pres. Request } & \multicolumn{5}{|c|}{ HPCC PCAs by 1996 Estimated } \\
\hline & $\begin{array}{l}\text { FY 95 } \\
\text { Actual }\end{array}$ & $\begin{array}{l}\text { FY 96 } \\
\text { Pres. }\end{array}$ & $\begin{array}{c}\text { FY } 96 \\
\text { Est. }\end{array}$ & $\begin{array}{l}\text { FY } 97 \\
\text { Rast }\end{array}$ & HECC & LSN & HCS & HuCS & ETHR & HECC & LSN & HCS & HuCS & ETHR \\
\hline AskERIC Service & 0.75 & 1.25 & 0.34 & 1.00 & & & & & 1.00 & & & & & 0.34 \\
\hline OERI Institutional Communications Network (INET) & & 1.00 & 0.93 & 1.90 & & & & & 1.90 & & & & & 0.93 \\
\hline Regional Education Laboratory Program & 1.42 & 1.50 & 1.50 & 1.50 & & & & & 1.50 & & & & & 1.50 \\
\hline Teacher Networking Project & 13.21 & 1.80 & 13.21 & & & & & & & & & & & 13.21 \\
\hline $\begin{array}{l}\text { National Institute on Disability and Rehabilitation } \\
\text { Pecearch }\end{array}$ & 11.40 & 11.40 & 11.40 & 11.40 & & & & 11.40 & & & & & 11.40 & \\
\hline National Parents Information Network & & & 0.20 & 0.23 & & & & & 0.23 & & & & & 0.20 \\
\hline ERIC Clearinghouses & 1.20 & & 1.35 & 1.98 & & & & & 1.98 & & & & & 1.35 \\
\hline Totals: & 27.98 & 16.95 & 28.93 & 18.01 & & & & 11.40 & 6.61 & & & & 11.40 & 17.53 \\
\hline
\end{tabular}


AskERIC is an online information service at the Educational Resources Information Center (ERIC) available free of charge to teachers, parents, and students. AskERIC provides its users with customized information on a vast array of educational topics, delivered via the Internet and commercial services such as America Online. Using state-of-the-art technology, AskERIC also provides a personal touch through trained specialists available to assist educators and parents in their search for information.

AskERIC has four components: a question answering (Q\&A) service, the AskERIC Virtual Library, the National Parent Information Network, and a research and development $(\mathrm{R} \& \mathrm{D})$ program.

For the Q\&A service, information specialists draw on the resources of the Internet and the ERIC system to provide answers-within 48 hours-to any question about education. Users can try the Q\&A service by sending a question via e-mail to AskERIC@ericir.syr.edu.

The AskERIC Virtual Library is a collection of education resources that teachers have requested. These materials include lesson plans, print, and video materials (from organizations such as CNN, PBS and the Discovery Channel), research summaries, digests, Infoguides on key educational topics, frequently-asked questions, and discussion groups for practicing educators and librarians. This is immediately available electronically.

The National Parent Information Network (NPIN)_developed in conjunction with the National Urban League, local housing authorities, and

PrairieNet-is an online collection of materials to help parents better support their children's educational, physical, and societal development.

AskERIC R\&D applies emerging tools and technologies to deliver services and provide on-demand access to a full range of electronic media. Currently, the AskERIC R\&D team is working on: expanding the AskERIC Virtual Library to provide image, sound, and video resources; providing free Internet access to the ERIC bibliographic database using friendly, high performance retrieval software; creating a full-text electronic collection of the documents in the ERIC database; and, experimenting with new Internet products to ascertain their value for serving educators.

\section{Milestone}

Changes

FY 1995 Actual Milestones

Completed ERIC bibliographic database available on Internet with capacity for 200 simultaneous users and state-of-the-art interface.

Full text of the documents indexed in the ERIC database in 1995 available online in image database service.

$\vec{w}$

w
AskERIC became truly nationwide (worldwide?)

\section{FY 1996 Estimated Milestones}

Will dramatically expand access to multimedia and hypermedia materials.

AskERIC will harness a nationwide network of information specialists, using two-way interactive elecommunications, to serve the needs of teachers, administrators, parents and students.

\section{FY 1997 Agency Request Milestones}

ERIC will continue to fulfill its mission of maintaining, and providing access to, the world's largest electronic bibliographic database of education-related publications and documents

through a national system of specialist clearing houses. The ERIC system synthesizes education information and disseminates it in print and electronic formats. The ERIC system provides access to its network of bibliographic information to educators, policy makers, parents and the general public. It provides access to the database clearinghouses via mail, telephone, and electronic routes. It maintains a question and answer service via the Internet.

\section{Budget (\$ M)}

\begin{tabular}{|c|c|c|}
\hline \multicolumn{2}{|c|}{ FY 95 Act } & 0.75 \\
\hline \multicolumn{2}{|c|}{ FY 96 Pres. } & 1.25 \\
\hline \multicolumn{2}{|c|}{ FY 96 Est. } & 0.34 \\
\hline \multicolumn{2}{|c|}{ FY 97 Rqst. } & 1.00 \\
\hline \multicolumn{3}{|c|}{$\begin{array}{c}\text { Program } \\
\text { Component } \\
\text { Areas }\end{array}$} \\
\hline & FY 96 & FY 97 \\
\hline \multicolumn{3}{|l|}{ HECC } \\
\hline \multicolumn{3}{|l|}{ LSN } \\
\hline \multicolumn{3}{|l|}{ HCS } \\
\hline \multicolumn{3}{|l|}{ HuCs } \\
\hline ETHR & 0.34 & 1.00 \\
\hline
\end{tabular}

Agency Ties

\begin{tabular}{|c|c|}
\hline DARPA & \\
\hline NSF & \\
\hline DOE & \\
\hline NASA & \\
\hline NIH & \\
\hline NSA & \\
\hline NIST & \\
\hline NOAA & \\
\hline EPA & \\
\hline ED & \\
\hline AHCPR & \\
\hline VA & \\
\hline
\end{tabular}

December 20, 1996 
The Institutional Communications Network (INet) was established in FY 93 by the Department of Education's Office of Educational Research and $\checkmark$ Improvement (OERI) to use the emerging National Information Infrastructure to accomplish the interrelated goals of: (1) improving collaboration and

A information sharing among the education R\&D institutions funded by OERI, including the Regional Educational Laboratories, National Educational Resed \& Development Centers, Educational Resources Information Center (ERIC), and National Diffusion Network; and (2) disseminating relevant and useful research, statistics, information, products, and publications developed through or supported by the Department to schools, educators, parents, and policy makers throughout the United States.

In FY 94, INet initiated public access Gopher, FTP, and World Wide Web servers, which have rapidly become important resources to the education community. Although INet initially concentrated on disseminating OERI's research and statistical information, its coverage has expanded to include extensive information from other Department offices--press releases, grant announcements, and libraries of information about major initiatives such as GOALS 2000, Technology, School-to-Work, and Elementary and Secondary Education Schoolwide Programs. Dissemination activities are closely coordinated with other Department-funded entities that sponsor Internet services, including AskERIC, the Eisenhower National Clearinghouse for Mathematics and Science Education, and the Regional Educational Laboratories.

The GOALS 2000 legislation calls for increased emphasis on electronic networking and dissemination to support education reform. INet will continue to play a key role in the Department's efforts to make a significant, high quality collection of education information available on-line for the rapidly growing number of educators who are connecting to the NII.

\section{Milestone}

Changes

\section{FY 1995 Actual Milestones}

Planed and began to implement core set of searchable databases, including current $R \& D$ activities, promising and exemplary programs, education statistics, and Department publications and products with links to on-line full text and on-line ordering.

Implemented community exchange service to support interchange and discussion of ideas by communities engaged in education reform.

Expanded participation of other Department's programs; coordinate linkages and organization of on-line libraries with other sites and systems. continued to explore and test enhancements to Mosaic useful to K-12 education and users with disabilities.

Pilot tested enhanced document and data formats and their usability by teachers, administrators, parents, and students.

\section{FY 1996 Estimated Milestones}

Continue implementation of core set of searchable databases.

Enhance services to exploit emerging data, retrieval, and interface technology; expand content to incorporate enhanced document and data formats. Support Department's integration of

clearinghouses, categorical and comprehensive technical assistance centers into a coordinated unified customer service/technical assistance system.

Expand participation of other Departmen programs; coordinate linkages and organization of , on-line libraries with other sites and systems.

s

\section{FY 1997 Agency Request Milestones}

The primary focus of the INet program will continue to be linking the National Library of Education, OERI-funded institutions, and other Department of Education services with schools, educators, parents, and policy makers. INet manages the Internet and World Wide Web presence of the National Library of Education and the Department of Education. Improvements are planned in two areas: Providing increased access through the Internet node (gopher and WWW) to the National Library's repository of information about Department programs, projects, publications, and statistics by beginning the development of a digital library; and actively disseminating syntheses of research and development findings and other materials through electronic networking.

\section{Budget (\$ M)}

\begin{tabular}{|c|c|}
\hline FY 95 Act & \\
\hline FY 96 Pres. & 1.00 \\
\hline FY 96 Est. & 0.93 \\
\hline FY 97 Rqst. & 1.90 \\
\hline
\end{tabular}

Program

Component

Areas

\begin{tabular}{|l|l|l|}
\hline & FY 96 & FY 97 \\
\hline HECC & & \\
\hline LSN & & \\
\hline HCS & & \\
\hline HuCS & & \\
\hline ETHR & 0.93 & 1.90 \\
\hline
\end{tabular}

Agency Ties

\begin{tabular}{|c|c|}
\hline DARPA & \\
\hline NSF & Partner \\
\hline DOE & \\
\hline NASA & \\
\hline NIH & \\
\hline NSA & \\
\hline NIST & \\
\hline NOAA & \\
\hline EPA & \\
\hline ED & \\
\hline AHCPR & \\
\hline VA & \\
\hline
\end{tabular}

December 20, 1996 


\begin{tabular}{|c|c|c|}
\hline ED & Regional Education Laboratory Program & Budget Code: \\
\hline
\end{tabular}

The Regional Educational Laboratories conduct applied research and development, disseminate information, and provide technical assistance that supports educational improvement. Their principal clients are educators and policy makers. The ten Regional Educational Laboratories also work together as a network on problems that affect several regions. Under the direction of a representative governing board, each laboratory serves clients in a defined region consisting of four to seven states. Using the best available knowledge, the Laboratories identify solutions to educational problems, try new approaches,

furnish research results and publications, and provide training to teachers and administrators. The laboratories support school restructuring with major efforts in curriculum, instruction, and assessment.

There is a growing effort by the Labs to use technology to link educators in schools and other educational agencies with educational R\&D results. The Labs have created a Technology Task Force, which is developing an Internet-based communication and information system called the National Educational R\&D Network. The Network will support the Labs' collaboration, dissemination, and constituent support activities. The goals of this network are: 1) To provide the staff of regional laboratories with the capabilities of electronic communication and data and information sharing for intra-Lab communication and collaboration purposes; 2) To provide clients of the Regional Educational Laboratories with easy access to a wide range of information and data services; 3 ) To provide for electronic communication, dissemination, and collaboration between individual Regional Educational Laboratories and their clients and constituents.

Milestone

Changes

FY 1995 Actual Milestones

The tenth Lab Unix system and Internet connection was completed.

The National Education R\&D Network gopher was made publicly available.

The pilot test of the Panda interface and system was completed.

Gopher menus were available for nine Labs.

Plans for Phase III system development and evaluation were completed. Phase III will focus on integrating technology in all laboratory programs and developing new technology products and services for clients.

A graphical system in World Wide Web

(WWW) as a parallel to the gopher based menu was completed.

The evaluation report of the Phase II

(Internet-based) Lab Network was completed.

\section{FY 1996 Estimated Milestones}

Complete work on the Phase III plan and evaluation.

Continue support for projects funded under the laboratory contract and increased connectivity with constituents and OERI.

$\vec{v}$

\section{FY 1997 Agency Request Milestones}

The laboratories assist educators and policy makers in their efforts to implement effective school reforms and solve State and local

education problems. Each laboratory will promote widespread access to information regarding research and best practice as well as connectivity to establish communities of learners; and use advanced technologies as an integral method of their operations.

Develop models of comprehensive, effective reform that are used to show States and local school districts how to implement successful programs in which all students achieve at high levels, and in which technology is an integral part of the instructional environment.
Budget (\$ M)

FY 95 Act

FY 96 Pres. 1.42

FY 96 Est.

1.50

FY 97 Rqst. 1.50

Progr

1.50

Program

Component

Areas

FY 96 FY 97

\begin{tabular}{|l|l|l|}
\hline HECC & & \\
\hline LSN & & \\
\hline HCS & & \\
\hline HuCS & & \\
\hline ETHR & 1.50 & 1.50 \\
\hline
\end{tabular}

\section{Agency Ties}

\begin{tabular}{|c|c|}
\hline DARPA & \\
\hline NSF & \\
\hline DOE & \\
\hline NASA & \\
\hline NIH & \\
\hline NSA & \\
\hline NIST & \\
\hline NOAA & \\
\hline EPA & \\
\hline ED & \\
\hline AHCPR & \\
\hline VA & \\
\hline
\end{tabular}




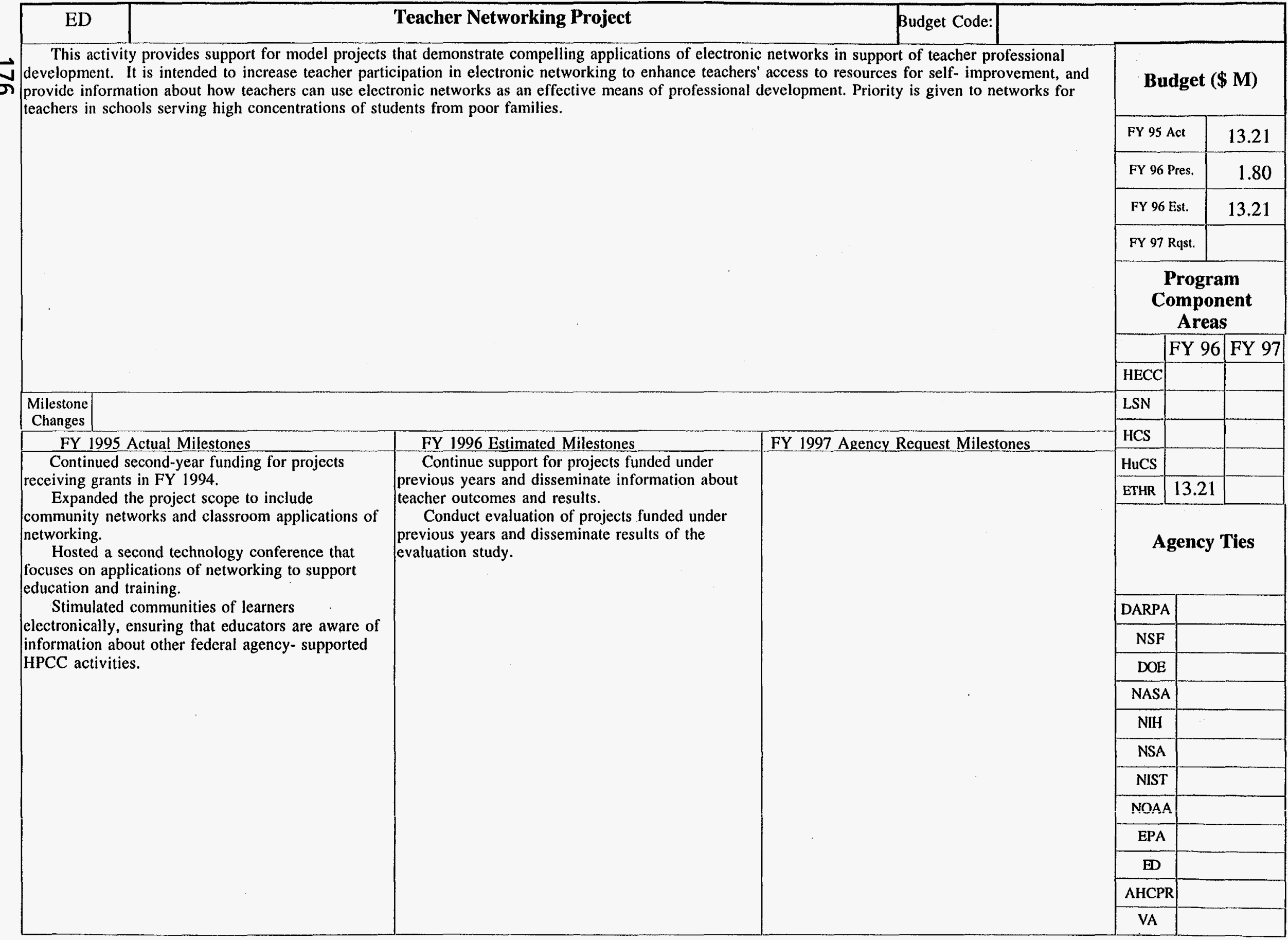


The Education Department's National Institute on Disability and Rehabilitation Research, within the office of Special Education and Rehabilitative Services, funds several programs with projects related to High Performance Computing and Communication. These include the Rehabilitation Engineering Research Centers (RERC) program, the Technology-Related Assistance program (TRA), the Research and Demonstration program, the Field Initiated Research program, and interagency programs.

RERCs conduct coordinated programs of advanced engineering or technological research: to develop and test new engineering solutions to problems confronting individuals with disabilities; to develop systems for the exchange of technical and engineering information; and to improve the distribution of technological devices and equipment to individuals with disabilities. These Centers have been established in the following areas supporting universal acces to HPCC technology: Adaptive Computers and Information Systems; Augmentative and Alternative Communication; Hearing Enhancement and Assistive Devices; Technology for Blind, Visually Impaired, and Multihandicapped Individuals; and for planning purposes only, Universal Access and

Telecommunications. Funding for these activities is about $\$ 3$ million per year.

The combined Research and Demonstration and Field Initiated Research programs yield about $\$ 1.7$ million per year in HPCC relevant research activities The TRA program involves 52 entities (primarily states) with programs of systems change that include personnel, equipment, and a system to generate database information that could be network accessible. Approximately $14 \%$ of the funding for the TRA program or $\$ 4.9$ million per year is allocated to this activity. Dissemination and Utilization activities account for about $\$ 2.5$ million per year allocated to production of information available for network access primarily through contract support of the National Rehabilitation Information Center (NARIC). NARIC collects the results of federally funded research projects related to disability and rehabilitation research.

Milestone

Changes

FY 1995 Actual Milestones

Enhancement of continuing research centers and programs to include a focus on ethnic and cultural diversity and increased participation of persons with disabilities in policy research.

Established two major research teams to pursue work in technology related to the National Information Infrastructure access and productive use

by persons with disabilities.

\section{FY 1996 Estimated Milestones}

Demonstrate an enhanced capability to deliver useful government information to persons with disabilities.

\section{FY 1997 Agency Request Milestones}

NIDR will continue funding of 15 continuing and 1 new Rehabilitation Engineering Research Centers (RERCs). These centers support programs designed to conduct research, demonstration, and training activities. RERCs focus on issues dealing with rehabilitation technology, including rehabilitation engineering and assistive technology devices and services.$$
\text { ( }
$$

Budget (\$ M)

\begin{tabular}{|c|c|}
\hline FY 95 Act & 11.40 \\
\hline FY 96 Pres. & 11.40 \\
\hline FY 96 Est. & 11.40 \\
\hline FY 97 Rqst. & 11.40 \\
\hline
\end{tabular}

Program

Component

Areas$$
\text { HEC }
$$

\begin{tabular}{|l|l|l|}
\hline LSN & & \\
\hline HCS & & \\
\hline HuCS & 11.40 & 11.40 \\
\hline ETHR & & \\
\hline
\end{tabular}

Agency Ties

\begin{tabular}{|c|c|}
\hline DARPA & \\
\hline NSF & Partner \\
\hline DOE & \\
\hline NASA & \\
\hline NIH & \\
\hline NSA & \\
\hline NIST & \\
\hline NOAA & \\
\hline EPA & \\
\hline ED & \\
\hline AHCPR & \\
\hline VA & Partner \\
\hline
\end{tabular}

December 20, 1996 


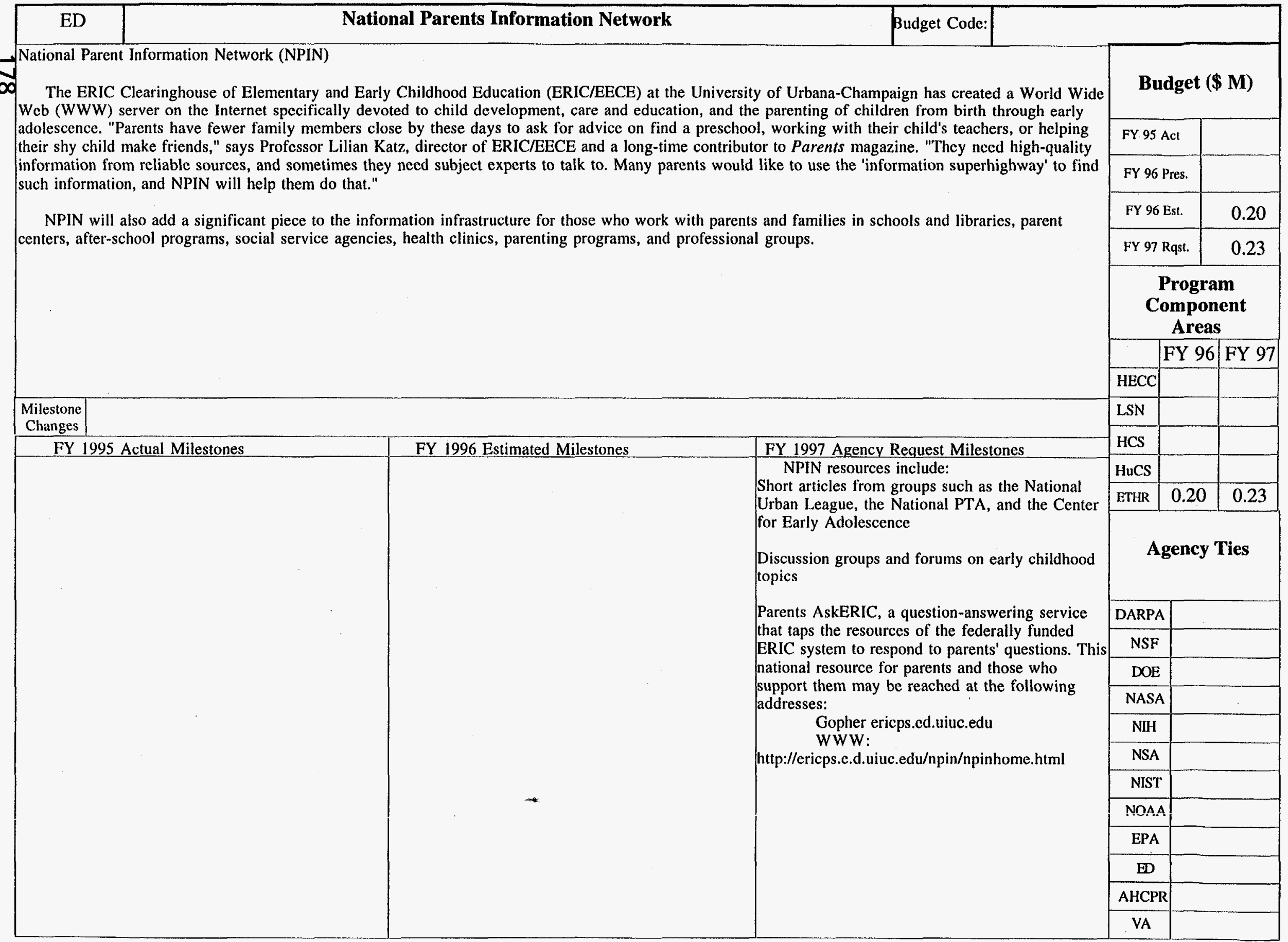




\begin{tabular}{|c|c|c|}
\hline ED & ERIC Clearinghouses & Budget Code: \\
\hline
\end{tabular}

The National Library of Education with the Office of Educational Research and Improvement administers the Education Resources Information Center (ERIC), which includes 16 clearinghouses that synthesize and disseminate education information, and is responsible for various other dissemination efforts, including dissemination through electronic networking and other technologies.

ERIC will continue to fulfill its mission of maintaining, and providing access to, the world's largest electronic bibliographic database of education-related publications and documents through a national system of specialist clearinghouses. Although OERI has begun exploring the inclusion of published books in the ERIC system, it remains primarily a resource for documents and publications not readily available elsewhere, providing significant services to classroom teachers and other users who do not have ready access to large reference libraries. The ERIC system synthesizes education information and disseminates it in print and electronic formats. It also provides information services to individuals and organizations in cooperation with the National Library and

OERI-funded institutions. The ERIC system provides syntheses of research and development findings and access to its network of bibliographic information to educators, policy makers, parents, and the general public. It provides access to the database clearinghouses via mail, telephone, and electronic routes. It maintains and operates a question and answer service via the Internet. In addition to its 16 major clearinghouses, the system includes a network of adjunct, volunteer clearinghouses that provide services in narrow topical areas.

Budget (\$ M)

\begin{tabular}{|c|c|}
\hline FY 95 Act & 1.20 \\
\hline FY 96 Pres. & \\
\hline FY 96 Est. & 1.35 \\
\hline FY 97 Rqst. & 1.98 \\
\hline
\end{tabular}

Program

Component

Areas

FY 96 $\longdiv { \text { FY } 9 7 }$

Milestone

Changes

\begin{tabular}{|l|l|}
\hline FY 1995 Actual Milestones & FY 1996 Estimated Milestones \\
\hline & \\
\hline
\end{tabular}

FY 1997 Agency Request Milestones

OERI plans to improve the ERIC system in significant ways. First is an effort to integrate an standardize the information services, particularly electronic, of the clearinghouses so as to provide better customer service.

The bibliographic databases will be enhanced and full text dissemination capabilities will be added.

\section{Agency Ties}

\begin{tabular}{|c|c|}
\hline DARPA & \\
\hline NSF & \\
\hline DOE & \\
\hline NASA & \\
\hline NIH & \\
\hline NSA & \\
\hline NIST & \\
\hline NOAA & \\
\hline EPA & \\
\hline ED & \\
\hline AHCPR & \\
\hline VA & \\
\hline
\end{tabular}

December 20, 1996 


\section{Agency for Health Care Policy and Research}

$\vec{\infty}$ The Agency for Health Care Policy and Research (AHCPR) contribution to the High Performance Computing and Communication initiative fouses on health care technology applications of computer-based patient records, computerized clinical decision support systems, and patient care data standards. These HPCC activities are predominantly technology applications support for promoting the development and evaluation of systems to foster their economic and medical feasibility. The HPCC program at AHCPR advances information technology that can provide significant benefits to all Americans by improving the quality, appropriateness, and effectiveness of health care, and improving their access to health care.

\begin{tabular}{|c|c|c|c|c|c|c|c|c|c|c|c|}
\hline \multirow[b]{2}{*}{ Program Activity } & \multirow[b]{2}{*}{ Budget Account Code } & \multirow{2}{*}{$\begin{array}{c}\text { Partner/User } \\
\text { Agencies }\end{array}$} & \multicolumn{4}{|c|}{ Budget $(\mathrm{BA}, \$ \mathrm{M})$} & \multicolumn{5}{|c|}{ HPCCPCAs by 1997 Pres. Request } \\
\hline & & & $\begin{array}{l}\text { FY } 95 \\
\text { Actual }\end{array}$ & $\begin{array}{c}\text { FY } 96 \\
\text { Pres. }\end{array}$ & $\begin{array}{c}\text { FY } 96 \\
\text { Est. }\end{array}$ & $\begin{array}{l}\text { FY } 97 \\
\text { Rqst. }\end{array}$ & HECC & LSN & HCS & HuCS & ETHR \\
\hline Computer-Based Patient Records & & NIH & 4.10 & 8.40 & 3.20 & 4.20 & & & & 4.20 & \\
\hline & & Totals: & 4.10 & 8.40 & 3.20 & 4.20 & & & & 4.20 & \\
\hline
\end{tabular}




\section{Agency for Health Care Policy and Research}

\begin{tabular}{|c|c|c|c|c|c|c|c|c|c|c|c|c|c|c|c|}
\hline \multirow{2}{*}{\multicolumn{2}{|c|}{ Program Activity }} & \multicolumn{4}{|c|}{ Budget (BA, \$ M) } & \multicolumn{5}{|c|}{ HPCC PCAs by 1997 Pres. Request } & \multicolumn{5}{|c|}{ HPCC PCAs by 1996 Estimated } \\
\hline & & $\begin{array}{l}\text { FY } 95 \\
\text { Actual } \\
\end{array}$ & $\begin{array}{c}\text { FY } 96 \\
\text { Pres. }\end{array}$ & $\begin{array}{c}\text { FY } 96 \\
\text { Est }\end{array}$ & $\begin{array}{l}\text { FY } 97 \\
\text { Rgst }\end{array}$ & HECC & LSN & HCS & HuCS & ETHR & HECC & LSN & HCS & HuCS & ETHR \\
\hline Computer-Based Patient Records & & 4.10 & 8.40 & 3.20 & 4.20 & & & & 4.20 & & & & & 3.20 & \\
\hline & Totals: & 4.10 & 8.40 & 3.20 & 4.20 & & & & 4.20 & & & & & 3.20 & \\
\hline
\end{tabular}




\begin{tabular}{|c|c|c|}
\hline AHCPR & Computer-Based Patient Records & Budget Code: \\
\hline
\end{tabular}

The objective of the computer-based patient record program activity is to improve the uniformity, accuracy, and retrievability of data about patient care $\vec{\infty}$ in the community and to promote its use for improved clinical decisions. It requires the development of clinical data standards and the integration of

$N$ information systems in diverse locations within institutions and across institutions and health care providers. Testing the application of computer-based patient record systems, decision support algorithms, and knowledge servers in physicians' offices, hospitals, patients' homes and other locations is of national importance to bring rapidly the benefits of HPCC to the provider and consumer of health care throughout the U.S.

Milestone Fostered collaboration of the U.S. patient care data standards developers through AHCPR's support of the American National Institute's Healthcare Informatics Changes Standards Planning Panel.

FY 1995 Actual Milestones

Supported scientific inquiry into applied research relevant to an electronic medical record.

AHCPR and NLM collaborated to fund 8 cooperative agreement testbeds that show applications of the computer-based patient record. The first application is developing and testing a common medical terminology. These studies will support extending computer-based patient record systems and show their incorporation of guidelines, decision aids, expert systems, and reminder systems.

Supported investigations into the uses of automated patient care data for patient care, research, quality assessment, patient outcome analysis, and electronic transmission of such data among institutions and providers.

Supported development of and access to automated patient care data bases for research.

\section{FY 1996 Estimated Milestones}

Support private sector development and testing of clinical patient care data standards for: (1) the definition and coding of medical terms, (2) the content of specific data sets for decision making, and (3) the electronic exchange of patient care data Support the evaluation of electronic medical record applications to determine the extent to which they improve: clinical decision making; the delivery of health services; and patient outcomes. These

evaluations should include: (1) how such systems are received by health care providers and patients (2) how physician and patient behavior changes; and (3) how patient outcomes, productivity, and costs of care are affected.

\section{FY 1997 Agency Request Milestones}

Support research into the barriers to a successful installation of comprehensive health information systems, emphasizing the speed, cost and human factors that affect success-to accelerate the transfer of computer-based information technology.

Evaluate the medical effectiveness and economic impact (cost benefits) of automated clinical decision support systems in diverse heal care settings.
Budget (\$ M)

\begin{tabular}{|c|c|}
\hline FY 95 Act & 4.10 \\
\hline FY 96 Pres. & 8.40 \\
\hline FY 96 Est. & 3.20 \\
\hline FY 97 Rqst. & 4.20 \\
\hline
\end{tabular}

Program

Component

Areas

\begin{tabular}{|l|l|l|}
\hline & FY 96 & FY 97 \\
\hline HECC & & \\
\hline LSN & & \\
\hline HCS & & \\
\hline HuCS & 3.20 & 4.20 \\
\hline ETHR & & \\
\hline
\end{tabular}

\section{Agency Ties}

\begin{tabular}{|c|c|}
\hline DARPA & \\
\hline NSF & \\
\hline DOE & \\
\hline NASA & \\
\hline NIH & Partner \\
\hline NSA & \\
\hline NIST & \\
\hline NOAA & \\
\hline EPA & \\
\hline ED & \\
\hline AHCPR & \\
\hline VA & \\
\hline
\end{tabular}




\section{Department of Veterans Affairs}

The Department of Veteran Affairs (VA) operates the largest centrally-directed health care system in the United States through 171 VA medical centers (VAMC), 362 outpatient clinics, 129 nursing homes, and 35 domiciliaries. The VHA Medical Care Network is converting to 22 VISNs with a large number of access points. VHA automation systems are being adapted to support this business restructuring. The goal is to allow access to appropriate data from any access point independent of the location of that data.

The VA has a coordinated, integrated automation program whose purpose is to improve patient care and administrative operations by introducing advanced automation to deliver the right information to the right person at the right time in an economical manner. More effective administrative operations emphasize automated administrative functions, procurement, and electronic commerce. Improved patient care emphasizes making the correct information and services available electronically to the clinician on the wards where they make decisions. This information is currently drawn from the facility based integrated patient based information system, local area network CD-ROM data bases, and other VA facilities on the VA's Wide Area Network (WAN). Plans include increased accessibility to other WAN resources such as Medline and Internet based digital libraries.

Various aspects of the program focus on integrating technologies to enhance the existing computer based patient record, as well as to improve methods of data capture, data presentation, and inter-facility data exchange. The projects concentrate on the technical areas of workstations, information system architectures, local and wide area networks, and appropriate security and privacy alternatives. Standards based solutions will be necessary to

\begin{tabular}{|c|c|c|c|c|c|c|c|c|c|c|c|}
\hline \multirow[b]{2}{*}{ Program Activity } & \multirow[b]{2}{*}{ Budget Account Code } & \multirow{2}{*}{$\begin{array}{c}\text { Partner/User } \\
\text { Agencies }\end{array}$} & \multicolumn{4}{|c|}{ Budget (BA, \$ M) } & \multicolumn{5}{|c|}{ HPCCPCAs by 1997 Pres. Request } \\
\hline & & & $\begin{array}{l}\text { FY 95 } \\
\text { Actual } \\
\end{array}$ & $\begin{array}{l}\text { FY } 96 \\
\text { Pres. }\end{array}$ & $\begin{array}{l}\text { FY } 96 \\
\text { Est. }\end{array}$ & $\begin{array}{l}\text { FY } 97 \\
\text { Rqst. }\end{array}$ & HECC & LSN & HCS & HuCS & ETHR \\
\hline $\begin{array}{l}\text { Computerized Patient Record and } \\
\text { Telemedicine }\end{array}$ & & $\begin{array}{l}\text { NIH, NIST, } \\
\text { AHCPR }\end{array}$ & 14.00 & 14.00 & 1.40 & 1.80 & & 1.20 & 0.20 & 0.40 & \\
\hline Clinical Workstations and Medical Imaging & & $\begin{array}{l}\text { NIST, } \\
\text { AHCPR.... }\end{array}$ & 7.66 & 3.61 & 5.20 & 3.00 & & 2.00 & 0.60 & 0.40 & \\
\hline $\begin{array}{l}\text { Improve Telecommunications Infrastructure } \\
\text { and Internet Connectivity }\end{array}$ & & ARPA, NIST & 0.18 & 0.18 & 0.18 & 0.75 & & 0.50 & 0.25 & & \\
\hline $\begin{array}{l}\text { VA Hybrid Open Systems Technology (VA } \\
\text { HOST) }\end{array}$ & & AHCPR, NIST & 14.46 & 14.50 & 14.50 & 7.75 & 1.00 & 4.75 & 1.00 & 1.00 & \\
\hline VA/DoD Sharing & & NIST, AHCPR & 1.25 & 3.71 & 0.65 & 1.25 & & 1.00 & 0.25 & & \\
\hline & & Totals: & 37.55 & 36.00 & 21.93 & 14.55 & 1.00 & 9.45 & 2.30 & 1.80 & \\
\hline
\end{tabular}




\section{Department of Veterans Affairs}

$\vec{D}$

\begin{tabular}{|c|c|c|c|c|c|c|c|c|c|c|c|c|c|c|}
\hline \multirow[b]{2}{*}{ Program Activity } & \multicolumn{4}{|c|}{ Budget $(\mathrm{BA}, \$ \mathrm{M})$} & \multicolumn{5}{|c|}{ HPCC PCAs by 1997 Pres. Request } & \multicolumn{5}{|c|}{ HPCC PCAs by 1996 Estimated } \\
\hline & $\begin{array}{l}\text { FY } 95 \\
\text { Actual }\end{array}$ & $\begin{array}{c}\text { FY } 96 \\
\text { Pres. }\end{array}$ & $\begin{array}{c}\text { FY } 96 \\
\text { Est. }\end{array}$ & $\begin{array}{l}\text { FY } 97 \\
\text { Rast. }\end{array}$ & HECC & LSN & HCS & $\mathrm{HuCS}$ & ETHR & HECC & LSN & HCS & HuCs & ETHR \\
\hline Computerized Patient Record and Telemedicine & 14.00 & 14.00 & 1.40 & 1.80 & & 1.20 & 0.20 & 0.40 & & & 0.80 & 0.20 & 0.40 & \\
\hline Clinical Workstations and Medical Imaging & 7.66 & 3.61 & 5.20 & 3.00 & & 2.00 & 0.60 & 0.40 & & & 4.00 & 1.20 & & \\
\hline Improve Telecommunications Infrastructure and & 0.18 & 0.18 & 0.18 & 0.75 & & 0.50 & 0.25 & & & & 0.18 & & & \\
\hline VA Hybrid Open Systems Technology (VA HOST) & 14.46 & 14.50 & 14.50 & 7.75 & 1.00 & 4.75 & 1.00 & 1.00 & & 3.00 & 8.50 & 1.50 & 1.50 & \\
\hline VA/DoD Sharing & 1.25 & 3.71 & 0.65 & 1.25 & & 1.00 & 0.25 & & & & 0.65 & & & \\
\hline Totals: & 37.55 & 36.00 & 21.93 & 14.55 & 1.00 & 9.45 & 2.30 & 1.80 & & 3.00 & 14.13 & 2.90 & 1.90 & \\
\hline
\end{tabular}


The continued development of the VA computerized patient record will expand availability of patient data and images to health care providers.

Computerized records ensure that data will be available at the point of care and accessible throughout the distributed network of VA and Federal health care facilities where patients are treated.

Components of the record will include:

PDX - Exchange of patient demographics and health summary data between treating VA facilities;

Clinical Lexicon - Integration and application of national and international standard nomenclatures, e.g., NLM's UMLS, to bring precision to the communication of clinical data across different health care organizations;

Patient Care Encounter - A framework into which order information can be associated with a given visit. Orders can be linked to a given diagnosis or complaint and eventual outcome, so that the effectiveness of specific orders or order sets can be established;

Standards-based inter-application communications - Includes a messaging system, HL7, SQL, and controlled vocabularies. Implementing these national and international standards will permit the exchange of data among the public and private sectors;

Telemedicine - In FY 93 the National Library of Medicine funded a High Performance Computing and Communications contract to a consortium of West Virginia University, community, health care provider, and corporate institutions. The purpose of the 3-year award was to apply and evaluate advanced networking technologies to the delivery of health services in both rural and urban areas of West Virginia. At the end of FY 94 Veterans Affairs Medical Center (VAMC) Clarksburg joined as a participant in the statewide Mountaineer Doctor TV Network linking rural hospitals in West Virginia for

telemedicine and educational purposes.

\section{\begin{tabular}{c|c} 
Milestone \\
Changes
\end{tabular}}

FY 1995 Actual Milestones

Established WWW pages for publication of information related to Medical Informatics Standards Patient Data Exchange package that will enable

activities. This is a collaborative effort with AHCPR. directly merging of patient data from multiple Connected Clarksburg VAMC to the HPCC supported statewide Mountaineer Doctor TV Network linking rural hospitals in West Virginia for telemedicine and educational purposes.

Developed and tested the Patient Care Encounter (PCE)-the framework into which order information can be associated with a given visit. PCE links orders to a given diagnosis or complaint and the eventual outcome so that the effectiveness of specific orders or order sets can be established.

Developed and tested the HL7 Package-a basic tool set for use by all applications that will assist in implementing standards based interfaces between clinical applications.

\section{FY 1996 Estimated Milestones}

Develop and test Enhanced PDX- the Enhanced reating facilities.

Develop and test DHCP Request Broker which processes messages between the Workstation front end and the Clinical Data Base.

Conversion of customized medical device interfaces to standards based HL7 messages.

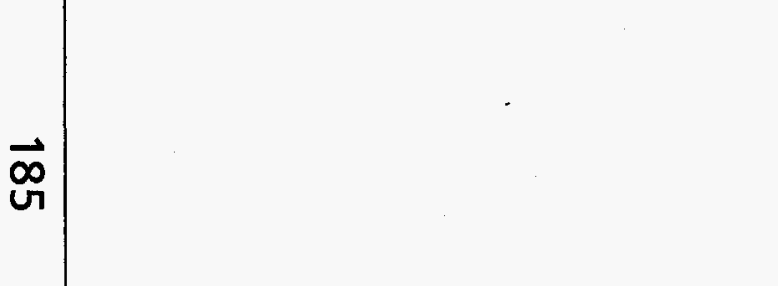

\section{FY 1997 Agency Request Milestones}

Enhance and test CPR (Computerized Patient Record) System.

Test the integration of Telemedicine Systems with the CPR.

Expand testing of VA Nationwide

Authentication, Authorization and Encryption

Services to allow secure transmission of medical data across unsecured telecommunication links such as public networks, phone systems and Internet.

Expand evaluation of options to use SQL as a mechanism of accessing medical data contain in disparate systems.

Develop and test an interactive voice respons system to enable access to information from existing VA computer systems 24 hours a day, 7 days a week.
Budget (\$ M)

\begin{tabular}{|c|c|}
\hline FY 95 Act & 14.00 \\
\hline FY 96 Pres. & 14.00 \\
\hline FY 96 Est. & 1.40 \\
\hline FY 97 Rqst. & 1.80 \\
\hline
\end{tabular}

Program

Component

Areas

FY 96 FY 97

HECC

\begin{tabular}{|l|l|l|}
\hline LSN & 0.80 & 1.20 \\
\hline HCS & 0.20 & 0.20 \\
\hline HuCS & 0.40 & 0.40 \\
\hline ETHR & & \\
\hline
\end{tabular}

Agency Ties

\begin{tabular}{|c|c|}
\hline DARPA & \\
\hline NSF & \\
\hline DOE & \\
\hline NASA & \\
\hline NIH & Partner \\
\hline NSA & \\
\hline NIST & Partner \\
\hline NOAA & \\
\hline EPA & \\
\hline ED & \\
\hline AHCPR & Partner \\
\hline VA & \\
\hline
\end{tabular}

December 20, 1996 
This program activity focuses on testing Clinical Workstations at pilot facilities. If these tests are successful, a non HPCC initiative could lead to nstallation of Clinical Workstations at all 171 VA facilities over a 4 year period beginning in 1995. There will be approximately three pilot sites implemented in FY 95, with additional sites being added in the subsequent years. Clinical workstations will extend and expand access to the computerized
patient record by linking workstations to VA's Decentralized Hospital Computer Program (DHCP). These multi-media workstations will be capable of pulling down information from the hospitals LAN, the VA's WAN, and networks such as the Internet that link the VA with the outside. Portable workstations will ensure access to information at the point of care.

The DHCP Integrated Medical Imaging Project currently is a model of a clinical workstation. This program has been operational at the Washington and Baltimore VAMCs for several years. Multiple types of true color and black-and-white medical images are captured and retrieved as an integrated part of the computerized patient record. Additional functionality is constantly being added to the system, and additional sites are installing the system.

FY 1995 Actual Milestones

Installed workstations and network at three sites

for initial testing of clinical record system. Martinsburg, Mountain Home, Boston, and

Milwaukee became operational.

Washington and Baltimore updated equipment

and supplemented the number of workstations.

Enhanced Text Data Capture workstations

became operational.
FY 1996 Estimated Milestones AHCPR Clinical Guidelines will be accessible electronically from the medical center wards at the Washington VAMC. Impact on clinicians activities will be evaluated.

Initial phase of national workstation installation Design and pilot test additional functionality for the clinical workstation.
FY 1997 Agency Request Milestones Develop and test GUI interface for existing Medical Imaging System.

Continue to convert applications from existing dumb terminal user interface to an open systems client serve architecture.

\section{Budget (\$ M)}

\begin{tabular}{|c|c|}
\hline FY 95 Act & 7.66 \\
\hline FY 96 Pres. & 3.61 \\
\hline FY 96 Est. & 5.20 \\
\hline FY 97 Rqst. & 3.00 \\
\hline
\end{tabular}

Program

Component

Areas

FY 96 FY 97

\section{}

LSN

4.00

HCS

HuCS

ETHR

1.20

1

\section{Agency Ties}

\begin{tabular}{|c|c|}
\hline DARPA & \\
\hline NSF & \\
\hline DOE & \\
\hline NASA & \\
\hline NIH & \\
\hline NSA & \\
\hline NIST & Partner \\
\hline NOAA & \\
\hline EPA & \\
\hline ED & \\
\hline AHCPR & Partner \\
\hline VA & \\
\hline
\end{tabular}

December 20, 1996 


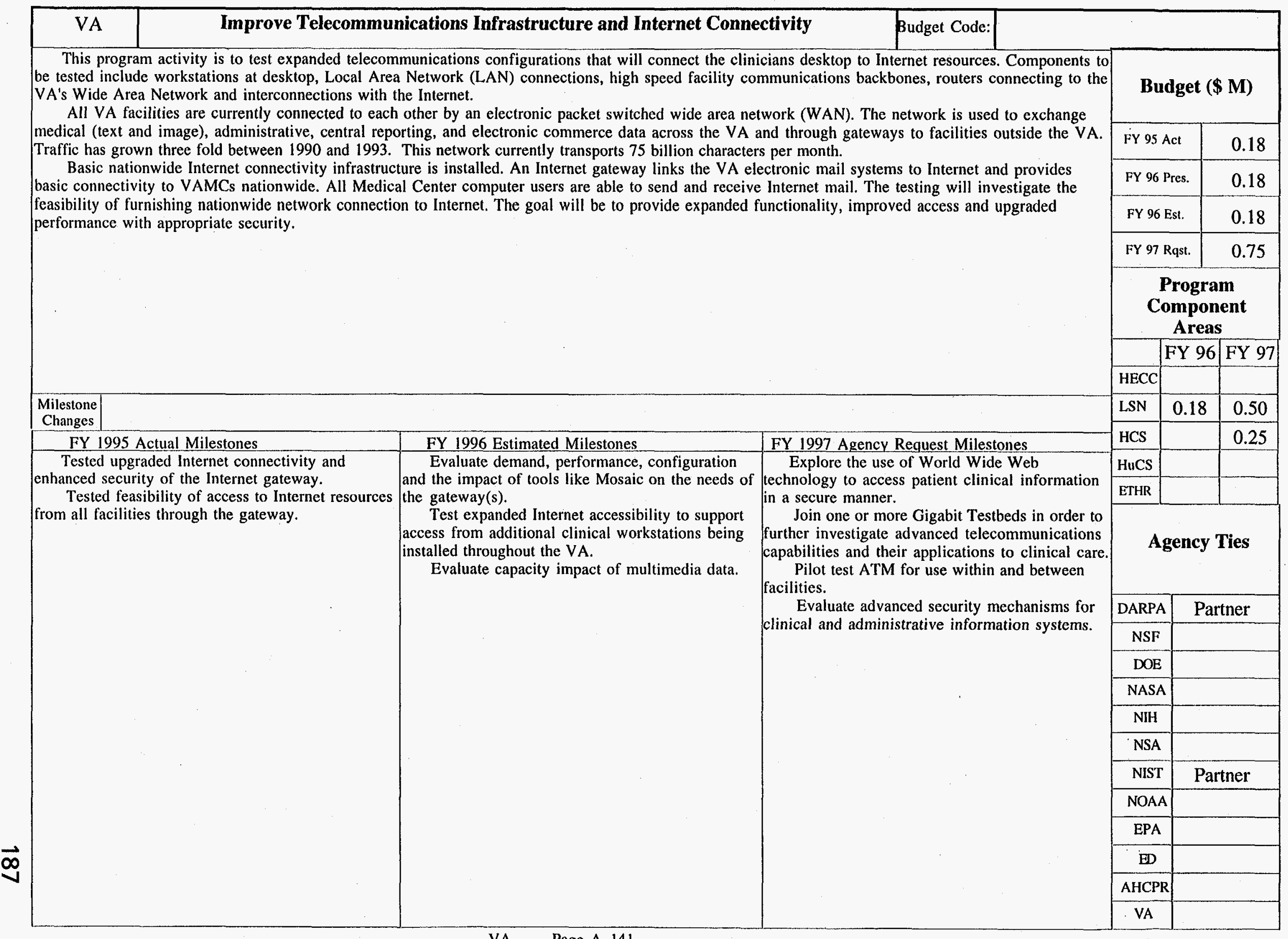


The purpose of the VA Hybrid Open Systems Technology (VA HOST) program activity is to investigate the feasibility of interfacing commercial (he VA's Integrated Hospital Information System called the Decentralized Hospital Computer Program (DHCP). This interfacing of commercial technologies is based on the development of standards-based interfaces in both the commercial technologies and in the VA DHCP.

The VA HOST program activity has enjoyed positive Congressional attention as a mechanism to support the VA in its efforts to continue to enhance the high level hospital information systems support currently installed at all VA Medical Centers. Traditionally, in-house developed, integrated software has provided the basis for the VA DHCP. As more commercial systems become available, and Open Systems standards and technologies evolve, investigation of commercial and Open System technologies for enhancement of the VA DHCP became the focus of this program activity. The VA HOST emphasizes a combination of the best of the in-house integrated system with the best of commercial technologies. Standards-based interfacing technologies are required in the VA HOST program.

\section{Milestone}

Changes

FY 1995 Actual Milestones

Initiated a second round of field-based project proposals.

Selected and funded 13 additional pilot projects Established a clearinghouse for VA HOST activities and standards-based integration with commercial technologies.

Designed and implemented the VA HOST migration procurement strategy.

Enhanced standards based integration paths to

VA DCHP for three additional commercial technologies.

Established a standards-based, Open systems Integration Laboratory for interoperability demonstrations.

\section{FY 1996 Estimated Milestones}

Initiate a third round of field-based project proposals.

Select and fund 10 additional pilot projects.

Complete technical assessments of five pilot projects.

Enhance standards based integration paths to HIS for three additional commercial technologies.

Initiate three project demonstrations in the Open Systems Integration Laboratory.

\section{FY 1997 Agency Request Milestones}

Initiate a fourth round of field-based project proposals.

Select and fund at least 10 additional pilot projects.

Complete technical assessments of five pilot projects.

Enhance standards based integration paths to VA HIS for three additional commercial technologies.

Test and select clinical and administrative projects for migration to multiple facilities.

Develop a prototype epidemiological server for use by clinicians and clinical researchers.

\section{Budget (\$ M)}

FY $95 \mathrm{Acl}$

FY 96 Pres.

FY 96 Est.

FY 97 Rqst.

7.75

Program

Component

Areas

FY 96 FY 97

HECC

3.00

LSN

HCS

$8.50 \quad 4.75$

HCS

HuCS

1.50

1.50

1.00

ETHR

1.00

Agency Thes

\section{Agency Ties}

\begin{tabular}{|c|c|}
\hline DARPA & \\
\hline NSF & \\
\hline DOE & \\
\hline NASA & \\
\hline NIH & \\
\hline NSA & \\
\hline NIST & Partner \\
\hline NOAA & \\
\hline EPA & \\
\hline ED & \\
\hline AHCPR & Partner \\
\hline VA & \\
\hline
\end{tabular}

December 20, 1996 


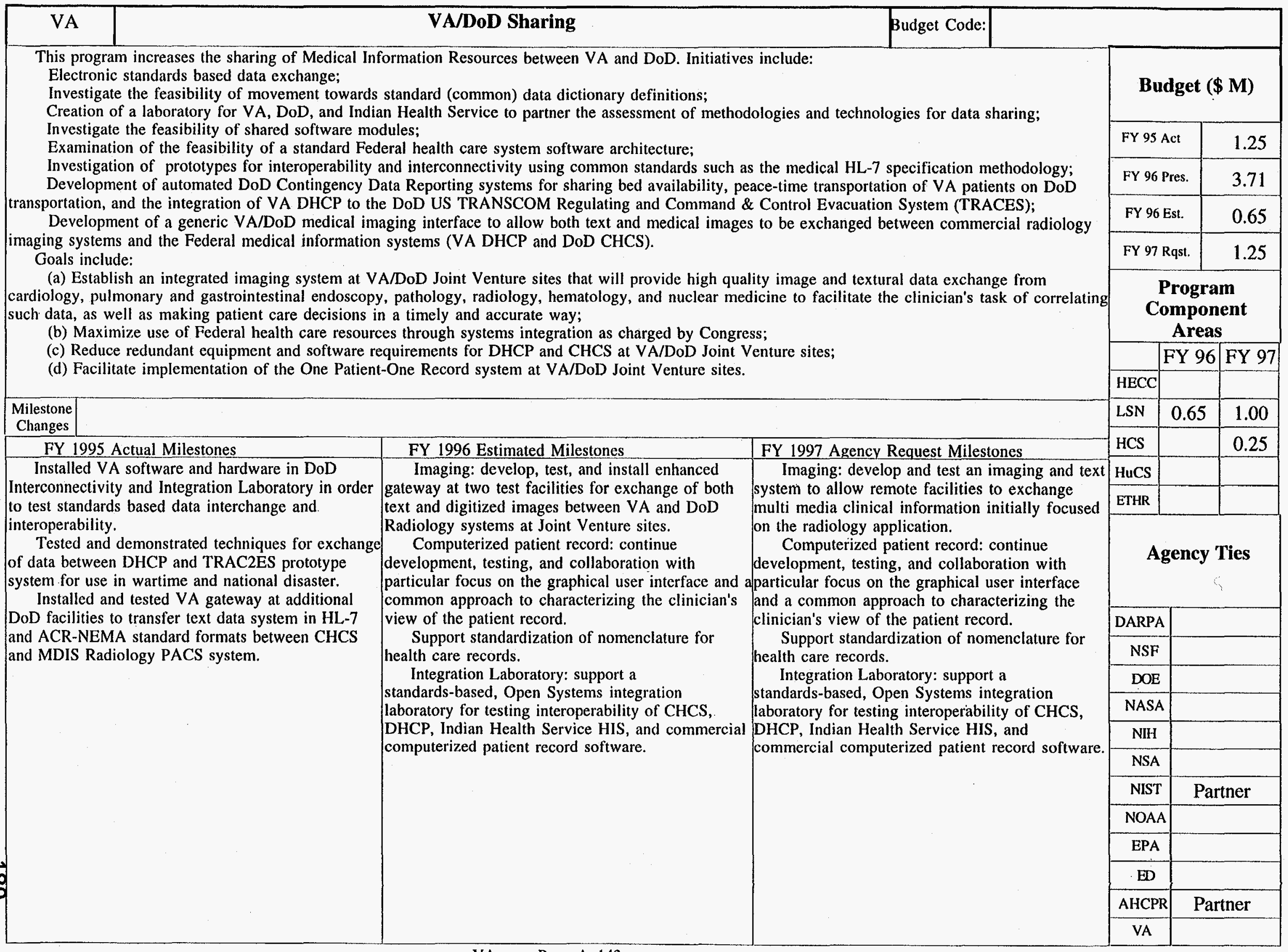


